PSI-PR-16-18

\title{
Higgs Boson Production and Decay at Hadron Colliders
}

\author{
Michael Spira \\ Paul Scherrer Institut, CH-5232 Villigen PSI, Switzerland
}

March 15, 2021

\begin{abstract}
Higgs physics at hadron colliders as the LHC is reviewed within the Standard Model (SM) and its minimal supersymmetric extension (MSSM) by summarizing the present state-of-the-art of theoretical predictions for the production cross sections and decay rates.
\end{abstract}

\section{Contents}

1 Introduction

1.1 Organization of the Review . . . . . . . . . . . . . . . . . . . . 2

1.2 Standard Model . . . . . . . . . . . . . . . . . . . . . . . 2

1.3 Minimal Supersymmetric Standard Model . . . . . . . . . . . . . . . . . 3

2 Higgs-Boson Decays $\quad 8$

2.1 Fermionic Higgs boson decays . . . . . . . . . . . . . . . . . . . . . . . . . . . . . . . 8

2.1.1 Standard Model .......................... 8

2.1.2 Minimal supersymmetric extension . . . . . . . . . . . . . . . 10

2.2 Higgs boson decays into intermediate gauge bosons . . . . . . . . . . . . . . . . . . . . . 13

2.2.1 Standard Model . . . . . . . . . . . . . . . . . . . . . 13

2.2.2 Minimal supersymmetric extension . . . . . . . . . . . . . . . 15

2.3 Higgs boson decays into gluons . . . . . . . . . . . . . . . . . . . . . . . . . 15

2.3.1 Standard Model . . . . . . . . . . . . . . . . . . . . 15

2.3.2 Minimal supersymmetric extension . . . . . . . . . . . . . . . . 19

2.4 Higgs boson decays into photons . . . . . . . . . . . . . . . . . . . . . . . . . . . 24

2.4.1 Standard Model . . . . . . . . . . . . . . . . . 24 24

2.4.2 Minimal supersymmetric extension . . . . . . . . . . . . . . . . 26

2.5 Higgs boson decays into photon and $Z$ boson and Dalitz decays . . . . . . . . . . . . . . 28

2.5.1 Standard Model . . . . . . . . . . . . . . . . . . . . 28

2.5.2 Minimal supersymmetric extension . . . . . . . . . . . . . . 32

2.6 Supersymmetric Higgs boson decays into Higgs particles . . . . . . . . . . . . . . . . . . 33

2.7 Supersymmetric Higgs boson decays . . . . . . . . . . . . . . . . . . . . . . . . . . 35

2.8 Branching ratios and total decay width . . . . . . . . . . . . . . . . . . . . . 40

2.8.1 Standard Model . . . . . . . . . . . . . . . . . . . . . 40

2.8.2 Minimal supersymmetric extension ................... 42 
3 Higgs-Boson Production

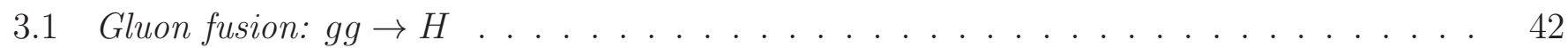

3.1.1 Standard Model ........................... . . . . 42

3.1.2 Minimal supersymmetric extension . . . . . . . . . . . . . . . . 50

3.2 Vector-boson fusion: $q q \rightarrow q q V^{*} V^{*} \rightarrow q q H \ldots \ldots \ldots \ldots \ldots$

3.2.1 Standard Model . . . . . . . . . . . . . . . . . . 52

3.2.2 Minimal supersymmetric extension . . . . . . . . . . . . . . . 54

3.3 Higgs-strahlung: $q \bar{q} \rightarrow V^{*} \rightarrow V H \ldots \ldots \ldots \ldots \ldots \ldots . \ldots \ldots \ldots$

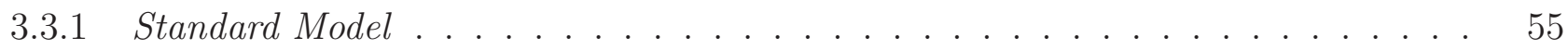

3.3.2 Minimal supersymmetric extension ................... . . . 56

3.4 Higgs bremsstrahlung off top and bottom quarks . . . . . . . . . . . . . . . . . . 57

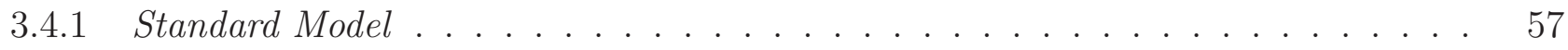

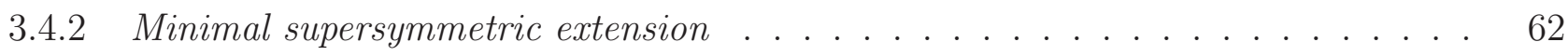

3.5 Summary of single-Higgs boson production cross sections . . . . . . . . . . . . . . . 66

3.5.1 Standard Model . . . . . . . . . . . . . . . . . . . 66

3.5.2 Minimal supersymmetric extension .................... 68

3.6 Higgs boson pair production . . . . . . . . . . . . . . . . . . . . . . . 68

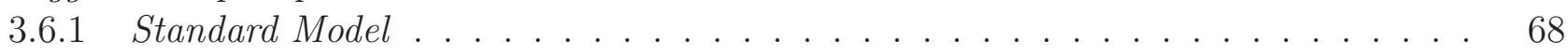

3.6.2 Minimal supersymmetric extension .................... 72

\section{Summary}

\section{Introduction}

\subsection{Organization of the Review}

In this work we will review all Higgs decay widths and branching ratios as well as all relevant Higgs boson production cross sections at the LHC within the SM and MSSM. Previous reviews can be found in Refs. [1. This work is a substantial update of Ref. [2].

The paper is organized as follows. This Section 1 will provide an introduction to the SM and MSSM Higgs sectors. In Section 2 we will review the decay rates of the SM and the MSSM Higgs particles providing explicit analytical results of the individual partial decay widths where possible. Section 3 will discuss the production cross sections for the Higgs bosons of the SM and MSSM and the impact of the numerical results on the profile of the Higgs bosons at the LHC. A summary will be given in Section 4.

\subsection{Standard Model}

The discovery of a resonance at the LHC [3] that is compatible with the Standard-Model (SM) Higgs boson [4] marked a milestone in particle physics. The existence of the Higgs boson is inherently related to the mechanism of spontaneous symmetry breaking [5] while preserving the full gauge symmetry and the renormalizability of the SM [6]. Its mass, the last missing parameter of the SM, has been measured to be $(125.09 \pm 0.24) \mathrm{GeV}$ [4]. The existence of the Higgs boson allows the SM particles to be weakly interacting up to high-energy scales without violating the unitarity bounds of scattering amplitudes [7]. This, however, is only possible for particular Higgs-boson couplings to all other particles so that with the knowledge of the Higgs-boson mass all its properties are uniquely fixed. The massive gauge bosons and fermions acquire mass through their interaction with the Higgs field that develops a finite vacuum expectation value in its ground state thus hiding the still unbroken electroweak gauge symmetry. The minimal model requires the introduction of one weak isospin doublet of the Higgs field and leads after 
spontaneous symmetry breaking to the existence of one scalar Higgs boson, while non-minimal Higgs sectors predict in general more than one Higgs boson.

Within the SM with one Higgs doublet the Higgs mass is constrained by consistency conditions as the absence of a Landau pole for the Higgs self-coupling up to high energy scales and the stability of the electroweak ground state [8]. If the SM is required to fulfill these conditions for energy scales up to the scale of Grand Unified theories (GUTs) of $\sim 10^{16} \mathrm{GeV}$ the Higgs mass is constrained between $129 \mathrm{GeV}$ and about $190 \mathrm{GeV}$ [9]. The last condition of vacuum stability can be relaxed by demanding the life-time of the ground state to be larger than the age of our universe [10]. This reduces the lower bound on the Higgs mass to about $110 \mathrm{GeV}$ [9]. The measured value of the Higgs mass indicates that our universe is unstable with a large lifetime far beyond the age of the universe.

If the SM is extended to the GUT scale, radiative corrections to the Higgs-boson mass tend to push the Higgs mass towards the GUT scale, if it couples to particles of that mass order. In order to obtain the Higgs mass at the electroweak scale the counter term has to be fine-tuned to cancel these large corrections thus establishing a very unnatural situation that requires a solution. This is known as the hierarchy problem [11]. Possible solutions are the introduction of supersymmetry (SUSY) [12, 13, a collective symmetry between SM particles and heavier partners as in Little Higgs theories [14] or an effective reduction of the Planck and GUT scales as in extra-dimension models at the TeV scale [15].

SM Higgs bosons are dominantly produced via the loop-induced gluon-fusion process $g g \rightarrow H$ with top quarks providing the leading loop contribution [16]. Other production processes as vectorboson fusion $q q \rightarrow q q H$ [17], Higgs-strahlung $q \bar{q} \rightarrow H+W / Z$ [18] and Higgs radiation off top quarks $g g, q \bar{q} \rightarrow t \bar{t} H$ [19] are suppressed by more than one order of magnitude. While the dominant gluonfusion process allows for the detection of the Higgs boson in the rare decay modes into $\gamma \gamma$, four charged leptons and $W$-boson pairs, other decay modes are only detectable in the subleading production modes as e.g. the main Higgs-boson decay into bottom quarks in strongly boosted Higgs-strahlung [20] or the Higgs-boson decay into $\tau$-leptons in the vector-boson-fusion process [21].

\subsection{Minimal Supersymmetric Standard Model}

Supersymmetric extensions of the SM are motivated by providing a possible solution to the hierarchy problem if the supersymmetric particles appear at the few-TeV scale [22]. Supersymmetry connects fermionic and bosonic degrees of freedom and thus links internal and external symmetries [12]. It is the last possible symmetry-type of $S$-matrix theories, i.e. the last possible extension of the Poincaré algebra. The minimal supersymmetric extension of the SM (MSSM) predicts the Weinberg angle in striking agreement with experimental measurements if embedded in a GUT [23. Moreover, it allows to generate electroweak symmetry breaking radiatively [24] and yields a candidate for Dark Matter if $R$-parity is conserved [25] which renders the lightest supersymmetric particle stable. Finally it increases the proton lifetime beyond experimental bounds in the context of supersymmetric GUTs [23, 26].

The MSSM requires the introduction of two isospin doublets of Higgs fields in order to maintain the analyticity of the superpotential and the anomaly-freedom with respect to the gauge symmetries [26, 27]. Moreover, two Higgs doublets are needed for the generation of the up- and down-type fermion masses. The Higgs sector is a Two-Higgs-Doublet model (2HDM) of type II. The mass eigenstates consist of a light $(h)$ and heavy $(H)$ scalar, a pseudoscalar $(A)$ and two charged $\left(H^{ \pm}\right)$states. The self-interactions of the Higgs fields are entirely fixed in terms of the electroweak gauge couplings so that the self-couplings are constrained to small values. This leads to an upper bound on the light scalar Higgs mass that has to be smaller than the $Z$-boson mass $M_{Z}$ at leading order (LO). This is, however, broken by radiative corrections, which are dominated by top-quark-induced contributions [28]. The parameter $\operatorname{tg} \beta$, defined as the ratio of the two vacuum expectation values of the scalar Higgs fields, will in general be assumed to be in the range $1<\operatorname{tg} \beta<m_{t} / m_{b}$, where $m_{t}\left(m_{b}\right)$ denotes the top (bottom) mass, consistent with the assumption that the MSSM is the low-energy limit of a supergravity model 
[29] and to avoid non-perturbative phenomena.

If the soft SUSY-breaking parameters do not contain any complex phases the input parameters of the MSSM Higgs sector at LO are generally chosen to be the mass $M_{A}$ of the pseudoscalar Higgs boson and $\operatorname{tg} \beta$. All other masses and the mixing angle $\alpha$ between the scalar CP-even Higgs states can be derived from these basic parameters (and the top mass and SUSY parameters, which enter through radiative corrections). The radiative corrections can be approximated by the parameter $\epsilon$, which grows with the fourth power of the top quark mass and logarithmically with the stop masses $m_{\tilde{t}_{1,2}}$, supplemented by terms originating from soft SUSY-breaking parameters, i.e. the trilinear coupling $A_{t}$, the higgsino mass $\mu$ and the third-generation squark mass $M_{S}$ with $X_{t}=A_{t}-\mu / \operatorname{tg} \beta$,

$$
\epsilon=\frac{3 G_{F}}{\sqrt{2} \pi^{2}} \frac{\bar{m}_{t}^{4}\left(M_{S}\right)}{\sin ^{2} \beta}\left\{\log \left(\frac{m_{\tilde{t}_{1}} m_{\tilde{t}_{2}}}{\bar{m}_{t}^{2}\left(M_{S}\right)}\right)+\frac{X_{t}^{2}}{M_{S}^{2}}\left(1-\frac{X_{t}^{2}}{12 M_{S}^{2}}\right)\right\}
$$

where $G_{F}$ denotes the Fermi constant and $\bar{m}_{t}\left(M_{S}\right)$ the $\overline{\mathrm{MS}}$ top mass at the scale $M_{S}$. These corrections are positive and reach a maximum (related to $X_{t}$ ) for $\left|X_{t}\right|=\sqrt{6} M_{S}$. They increase the squared mass of the light neutral Higgs boson $h$ to

$$
M_{h}^{2} \leq M_{Z}^{2} \cos ^{2} 2 \beta+\epsilon \sin ^{2} \beta
$$

In this approximation, the upper bound on $M_{h}$ is shifted from the tree level value $M_{Z}$ up to $\sim 145$ $\mathrm{GeV}$. The mass of the lightest scalar state $h$ is given by

$$
\begin{aligned}
M_{h}^{2}= & \frac{1}{2}\left[M_{A}^{2}+M_{Z}^{2}+\epsilon\right. \\
& \left.-\sqrt{\left(M_{A}^{2}+M_{Z}^{2}+\epsilon\right)^{2}-4 M_{A}^{2} M_{Z}^{2} \cos ^{2} 2 \beta-4 \epsilon\left(M_{A}^{2} \sin ^{2} \beta+M_{Z}^{2} \cos ^{2} \beta\right)}\right]
\end{aligned}
$$

The masses of the heavy neutral and charged Higgs bosons are determined by the sum rules (valid for this approximation),

$$
\begin{aligned}
M_{H}^{2} & =M_{A}^{2}+M_{Z}^{2}-M_{h}^{2}+\epsilon \\
M_{H^{ \pm}}^{2} & =M_{A}^{2}+M_{W}^{2}
\end{aligned}
$$

The effective mixing parameter $\alpha$ between the CP-even scalar Higgs states can be derived as

$$
\operatorname{tg} 2 \alpha=\operatorname{tg} 2 \beta \frac{M_{A}^{2}+M_{Z}^{2}}{M_{A}^{2}-M_{Z}^{2}+\epsilon / \cos 2 \beta}
$$

The radiative corrections to the MSSM Higgs sector have been calculated up to the two-loop level in the effective potential approximation and in the diagrammatic approach [28]. The leading corrections are also known at the three-loop level within the effective potential approach [30]. The corrections beyond next-to-leading order (NLO) are dominated by the QCD corrections to the top-quark-induced contributions. They decrease the upper bound on the light scalar Higgs mass $M_{h}$ by about $10 \mathrm{GeV}$ to $\sim 135 \mathrm{GeV}$, while leaving a residual uncertainty of $\sim 3 \mathrm{GeV}$ on the light scalar Higgs mass. In the context of increasing lower mass bounds for the supersymmetric particles, a resummation of large logarithms related to the SUSY-particle masses has been performed for the calculation of the MSSM Higgs-boson masses [31]. For large SUSY-particle masses this resummation may lead to effects of the order of $5 \mathrm{GeV}$ (or larger for small values of $\operatorname{tg} \beta$ ) on the light scalar Higgs mass.

For on-shell external Higgs bosons there is an additional relevant effect emerging from finite external Higgs momenta leading to an additional mixing between different types of Higgs bosons. This can be treated by the introduction of an external mixing matrix as discussed in Ref. [32] that ensures that the external Higgs bosons are defined as proper on-shell states. The numerical effects are sizeable for 
certain MSSM scenarios and have to be taken into account in all processes with external MSSM Higgs bosons.

The calculation of the radiative corrections has also been extended to the effective trilinear and quartic self-interactions of the MSSM Higgs bosons that are consistently defined at vanishing external momenta. The NLO corrections at $\mathcal{O}\left(\alpha_{t}\right)$ and $\mathcal{O}\left(\alpha_{b}\right)$ are available since a long time [33], where $\alpha_{t}=$ $G_{F} m_{t}^{2} /\left(\sqrt{2} \sin ^{2} \beta\right)$ and $\alpha_{b}=G_{F} m_{b}^{2} /\left(\sqrt{2} \cos ^{2} \beta\right)$. In the leading approximation in terms of the parameter $\epsilon$ the trilinear couplings of the neutral Higgs bosons are given up to $\mathcal{O}\left(\alpha_{t}\right)$ by 33 ]

$$
\begin{aligned}
\lambda_{h h h} & =3 \cos (2 \alpha) \sin (\beta+\alpha)+\frac{3 \epsilon}{M_{Z}^{2}} \frac{\cos ^{3} \alpha}{\sin \beta} \\
\lambda_{H h h} & =2 \sin (2 \alpha) \sin (\beta+\alpha)-\cos (2 \alpha) \cos (\beta+\alpha)+\frac{3 \epsilon}{M_{Z}^{2}} \frac{\sin \alpha \cos ^{2} \alpha}{\sin \beta} \\
\lambda_{H H h} & =-2 \sin (2 \alpha) \cos (\beta+\alpha)-\cos (2 \alpha) \sin (\beta+\alpha)+\frac{3 \epsilon}{M_{Z}^{2}} \frac{\sin ^{2} \alpha \cos \alpha}{\sin \beta} \\
\lambda_{H H H} & =3 \cos (2 \alpha) \cos (\beta+\alpha)+\frac{3 \epsilon}{M_{Z}^{2}} \frac{\sin ^{3} \alpha}{\sin \beta} \\
\lambda_{h A A} & =\cos (2 \beta) \sin (\beta+\alpha)+\frac{\epsilon}{M_{Z}^{2}} \frac{\cos \alpha \cos ^{2} \beta}{\sin \beta} \\
\lambda_{H A A} & =-\cos (2 \beta) \cos (\beta+\alpha)+\frac{\epsilon}{M_{Z}^{2}} \frac{\sin \alpha \cos ^{2} \beta}{\sin \beta}
\end{aligned}
$$

which have been normalized to $\sqrt{\sqrt{2} G_{F}} M_{Z}^{2}$. Similar but more involved expressions can be obtained for the quartic Higgs self-couplings. Some time ago the NLO corrections to the Higgs self-couplings have been extended by the next-to-next-to-leading order (NNLO) corrections of $\mathcal{O}\left(\alpha_{t} \alpha_{s}\right)$ [34], where $\alpha_{s}$ denotes the strong coupling constant. The radiative corrections are large in general, while the NNLO

\begin{tabular}{|c|c|c|c|c|}
\hline \multicolumn{2}{|l|}{$\Phi$} & $g_{u}^{\Phi}$ & $g_{d}^{\Phi}$ & $g_{V}^{\Phi}$ \\
\hline SM & $H$ & 1 & 1 & 1 \\
\hline \multirow[t]{3}{*}{ MSSM } & $h$ & $\cos \alpha / \sin \beta$ & $-\sin \alpha / \cos \beta$ & $\sin (\beta-\alpha)$ \\
\hline & $H$ & $\sin \alpha / \sin \beta$ & $\cos \alpha / \cos \beta$ & $\cos (\beta-\alpha)$ \\
\hline & $A$ & $1 / \operatorname{tg} \beta$ & $\operatorname{tg} \beta$ & 0 \\
\hline
\end{tabular}
part is of moderate size but reduces the theoretical uncertainties significantly to the few per-cent level.

The couplings of the neutral Higgs bosons to fermions and gauge bosons depend on the angles $\alpha$ and $\beta$. Normalized to the SM Higgs couplings, they are listed in Table 1. The pseudoscalar particle $A$ does not couple to gauge bosons at tree level, and its couplings to down (up)-type fermions are (inversely) proportional to $\operatorname{tg} \beta$. For large values of $\operatorname{tg} \beta$ the Yukawa couplings to (up) down-type quarks

Table 1: MSSM Higgs couplings to up- and down-type fermions and gauge bosons $(V=W, Z)$ relative to the corresponding SM couplings.

are (suppressed) enhanced and vice versa apart from the regions where the light (heavy) scalar is close to its upper (lower) mass bound, where their coupling factors approach the SM-coupling strengths (up to sign differences for the heavy scalar). The couplings of the light scalar Higgs particle approach the SM values for large pseudoscalar masses, i.e. in the decoupling regime. Thus it will be difficult to 
distinguish the light scalar MSSM Higgs boson from the SM Higgs particle in the region where all Higgs particles except the light scalar one are very heavy.

In addition to these coupling factors the bottom Yukawa coupling in particular is strongly modified by radiative corrections due to sbottom-gluino exchange in the genuine SUSY-QCD part and by stopchargino exchange in the leading SUSY-electroweak part, see Fig. 1. The leading contribution behaves

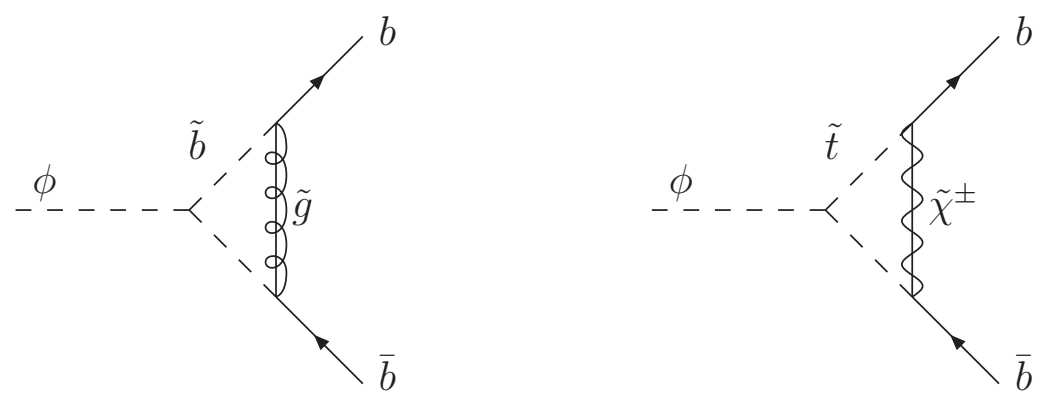

Figure 1: Feynman diagrams contributing to the bottom Yukawa couplings at $\mathcal{O}\left(\alpha_{s}\right)$ (left) and $\mathcal{O}\left(\lambda_{t}^{2}\right)$ (right).

as $\mathcal{O}\left(\mu m_{\tilde{g}} \operatorname{tg} \beta / M_{S U S Y}^{2} \alpha_{s} / \pi\right)$ (with the gluino mass $m_{\tilde{g}}$ ) and can thus be very large for large values of $\operatorname{tg} \beta$ [35]. In order to improve the perturbative results these contributions have to be resummed up to all orders. This can be done in the current-eigenstate basis involving the two Higgs doublets $\phi_{1}$ and $\phi_{2}$ that couple to down- and up-type quarks, respectively. The leading correction to the bottom Yukawa coupling emerges from a coupling of the 'wrong' doublet $\phi_{2}$ to the bottom quarks and can be discussed in terms of the effective Lagrangian

$$
\begin{aligned}
& \mathcal{L}_{e f f}=-\lambda_{b} \overline{b_{R}}\left[\phi_{1}^{0}+\frac{\Delta_{b}}{\operatorname{tg} \beta} \phi_{2}^{0 *}\right] b_{L}+h . c . \\
&=-m_{b} \bar{b}\left[1+i \gamma_{5} \frac{G^{0}}{v}\right] b-\frac{m_{b} / v}{1+\Delta_{b}} \bar{b}\left[g_{b}^{h}\left(1-\frac{\Delta_{b}}{\operatorname{tg} \alpha \operatorname{tg} \beta}\right) h\right. \\
&\left.\quad+g_{b}^{H}\left(1+\Delta_{b} \frac{\operatorname{tg} \alpha}{\operatorname{tg} \beta}\right) H-g_{b}^{A}\left(1-\frac{\Delta_{b}}{\operatorname{tg}^{2} \beta}\right) i \gamma_{5} A\right] b
\end{aligned}
$$

in the low-energy limit where the relations between current and mass eigenstates of the neutral Higgs components

$$
\begin{aligned}
\phi_{1}^{0} & =\frac{1}{\sqrt{2}}\left[v_{1}+H \cos \alpha-h \sin \alpha+i A \sin \beta-i G^{0} \cos \beta\right] \\
\phi_{2}^{0} & =\frac{1}{\sqrt{2}}\left[v_{2}+H \sin \alpha+h \cos \alpha+i A \cos \beta+i G^{0} \sin \beta\right]
\end{aligned}
$$

have been used and $G^{0}$ denotes the neutral would-be Goldstone component. The indices $L, R$ denote the chiralities of the bottom states, $\lambda_{b}$ the bottom Yukawa coupling of the MSSM Lagrangian and $v \approx 246$ $\mathrm{GeV}$ the $\mathrm{SM}$ vacuum expectation value. The leading contributions to the correction $\Delta_{b}$ at NLO are given by [35]

$$
\begin{aligned}
\Delta_{b} & =\Delta_{b}^{Q C D}+\Delta_{b}^{e l w, t}+\Delta_{b}^{e l w, 1}+\Delta_{b}^{e l w, 2} \\
\Delta_{b}^{Q C D} & =\frac{C_{F}}{2} \frac{\alpha_{s}}{\pi} m_{\tilde{g}} \mu \operatorname{tg} \beta I\left(m_{\tilde{b}_{1}}^{2}, m_{\tilde{b}_{2}}^{2}, m_{\tilde{g}}^{2}\right) \\
\Delta_{b}^{e l w, t} & =\frac{\lambda_{t}^{2}}{(4 \pi)^{2}} A_{t} \mu \operatorname{tg} \beta I\left(m_{\tilde{t}_{1}}^{2}, m_{\tilde{t}_{2}}^{2}, \mu^{2}\right)
\end{aligned}
$$




$$
\begin{aligned}
\Delta_{b}^{e l w, 1}=-\frac{\alpha_{1}}{12 \pi} M_{1} \mu \operatorname{tg} \beta\left\{\frac{1}{3} I\left(m_{\tilde{b}_{1}}^{2}, m_{\tilde{b}_{2}}^{2}, M_{1}^{2}\right)+\left(\frac{c_{b}^{2}}{2}+s_{b}^{2}\right) I\left(m_{\tilde{b}_{1}}^{2}, M_{1}^{2}, \mu^{2}\right)\right. \\
\left.+\left(\frac{s_{b}^{2}}{2}+c_{b}^{2}\right) I\left(m_{\tilde{b}_{2}}^{2}, M_{1}^{2}, \mu^{2}\right)\right\} \\
\Delta_{b}^{e l w, 2}=-\frac{\alpha_{2}}{4 \pi} M_{2} \mu \operatorname{tg} \beta\left\{c_{t}^{2} I\left(m_{\tilde{t}_{1}}^{2}, M_{2}^{2}, \mu^{2}\right)+s_{t}^{2} I\left(m_{\tilde{t}_{2}}^{2}, M_{2}^{2}, \mu^{2}\right)\right. \\
\left.+\frac{c_{b}^{2}}{2} I\left(m_{\tilde{b}_{1}}^{2}, M_{2}^{2}, \mu^{2}\right)+\frac{s_{b}^{2}}{2} I\left(m_{\tilde{b}_{2}}^{2}, M_{2}^{2}, \mu^{2}\right)\right\}
\end{aligned}
$$

where $C_{F}=4 / 3$ and $\lambda_{t}$ denotes the top Yukawa coupling and $\alpha_{1}=g^{\prime 2} / 4 \pi, \alpha_{2}=g^{2} / 4 \pi$ the electroweak gauge couplings. The masses $m_{\tilde{b}_{1,2}}$ and $m_{\tilde{t}_{1,2}}$ are the sbottom and stop masses, $\mu$ the higgsino mass parameter and $M_{1,2}$ the soft SUSY-breaking bino and wino mass parameters. The variables $s / c_{t, b}=$ $\sin / \cos \theta_{t, b}$ are related to the stop/sbottom mixing angles $\theta_{t, b}$. The function $I$ is generically defined as

$$
I(a, b, c)=\frac{a b \log \frac{a}{b}+b c \log \frac{b}{c}+c a \log \frac{c}{a}}{(a-b)(b-c)(a-c)}
$$

The effective Lagrangian of Eq. (7) can be parametrized in compact form as

$$
\mathcal{L}_{e f f}=-\frac{m_{b}}{v} \bar{b}\left[\tilde{g}_{b}^{h} h+\tilde{g}_{b}^{H} H-\tilde{g}_{b}^{A} i \gamma_{5} A\right] b
$$

with the effective (resummed) couplings

$$
\begin{aligned}
& \tilde{g}_{b}^{h}=\frac{g_{b}^{h}}{1+\Delta_{b}}\left[1-\frac{\Delta_{b}}{\operatorname{tg} \alpha \operatorname{tg} \beta}\right] \\
& \tilde{g}_{b}^{H}=\frac{g_{b}^{H}}{1+\Delta_{b}}\left[1+\Delta_{b} \frac{\operatorname{tg} \alpha}{\operatorname{tg} \beta}\right] \\
& \tilde{g}_{b}^{A}=\frac{g_{b}^{A}}{1+\Delta_{b}}\left[1-\frac{\Delta_{b}}{\operatorname{tg}^{2} \beta}\right]
\end{aligned}
$$

It should be noted that in the decoupling limit of large pseudoscalar mass $M_{A}$ the mixing angle factor $\operatorname{tg} \alpha$ approaches $-1 / \operatorname{tg} \beta$ such that $\tilde{g}_{b}^{h} \rightarrow g_{b}^{h} \rightarrow 1$ so that the light scalar Higgs boson still becomes SMlike. By means of power counting it can be shown that the Lagrangian of Eq. (77) is valid up to all orders in $\mu \operatorname{tg} \beta$ and thus $\Delta_{b}$ [36, 37, 38] and potentially sizeable $A_{b}$-contributions can be absorbed in a refined definition of $\Delta_{b}$ [37]. The rewriting in terms of mass eigenstates describes the proper resummation of these terms. Two-loop QCD corrections to the dominant $\Delta_{b}^{Q C D}$ and $\Delta_{b}^{e l w, t}$ contributions have been calculated. They modify the size by a moderate amount of about $10 \%$ and reduce the scale dependence considerably to the level of a few per-cent [38, 39] and thus yield a reliable prediction of the effective bottom Yukawa couplings. The couplings to all down-type fermions including the charged leptons can be dressed by the corresponding leading $\Delta_{f}$ terms analogously. They play a role for the strange and $\tau$ Yukawa couplings. While the $\Delta_{s}$ contributions can be obtained from $\Delta_{b}$ by straightforward replacements of the corresponding masses, the corrections for $\tau$ leptons are given explicitly by [35]

$$
\begin{aligned}
\Delta_{\tau}= & \Delta_{\tau}^{e l w, 1}+\Delta_{\tau}^{e l w, 2} \\
\Delta_{\tau}^{e l w, 1}=\frac{\alpha_{1}}{4 \pi} M_{1} \mu \operatorname{tg} \beta\{ & I\left(m_{\tilde{\tau}_{1}}^{2}, m_{\tilde{\tau}_{2}}^{2}, M_{1}^{2}\right)+\left(\frac{c_{\tau}^{2}}{2}-s_{\tau}^{2}\right) I\left(m_{\tilde{\tau}_{1}}^{2}, M_{1}^{2}, \mu^{2}\right) \\
& \left.+\left(\frac{s_{\tau}^{2}}{2}-c_{\tau}^{2}\right) I\left(m_{\tilde{\tau}_{2}}^{2}, M_{1}^{2}, \mu^{2}\right)\right\}
\end{aligned}
$$




$$
\begin{gathered}
\Delta_{\tau}^{e l w, 2}=-\frac{\alpha_{2}}{4 \pi} M_{2} \mu \operatorname{tg} \beta\left\{I\left(m_{\tilde{\nu}_{\tau}}^{2}, M_{2}^{2}, \mu^{2}\right)+\frac{c_{\tau}^{2}}{2} I\left(m_{\tilde{\tau}_{1}}^{2}, M_{2}^{2}, \mu^{2}\right)\right. \\
\left.+\frac{s_{\tau}^{2}}{2} I\left(m_{\tilde{\tau}_{2}}^{2}, M_{2}^{2}, \mu^{2}\right)\right\}
\end{gathered}
$$

where $s / c_{\tau}=\sin / \cos \theta_{\tau}$ is related to the $\tilde{\tau}$ mixing angle $\theta_{\tau}$ and $m_{\tilde{\tau}_{1,2}}, m_{\tilde{\nu}_{\tau}}$ denote the stau and tau sneutrino masses, respectively.

\section{$2 \quad$ Higgs-Boson Decays}

Higgs-boson couplings to SM particles grow with their corresponding masses so that for the SM Higgs boson its couplings to the intermediate gauge bosons $W, Z$ and to the top and bottom quarks as well as $\tau$-leptons are the most relevant ones for decay and production processes.

\subsection{Fermionic Higgs boson decays}

\subsubsection{Standard Model}

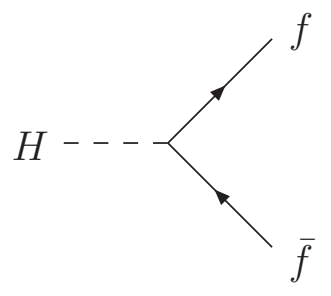

Figure 2: Feynman diagram describing $H \rightarrow f \bar{f}$ at lowest order.

The decay widths of the SM Higgs boson into fermion pairs mediated by the fermion Yukawa couplings (see Fig. 2) is given, for $M_{H}^{2} \gg m_{f}^{2}$, by [40, 41]

$$
\Gamma[H \rightarrow f \bar{f}]=\frac{N_{c} G_{F} M_{H}}{4 \sqrt{2} \pi} m_{f}^{2}\left[1+\delta_{\mathrm{QCD}}+\delta_{t}+\delta_{\text {mixed }}\right]\left(1+\delta_{\text {elw }}\right)
$$

with $N_{c}=1(3)$ for leptons (quarks), the Higgs-boson mass $M_{H}$ and the fermion mass $m_{f}$. In the

leptonic case we use the lepton pole mass, while for quarks we use the $\overline{\mathrm{MS}}$ quark mass $\bar{m}_{Q}\left(M_{H}\right)$. The QCD corrections for the decays into quark pairs can be expressed as [42, 43, 44]

$$
\begin{aligned}
\delta_{\mathrm{QCD}}= & 5.67 \frac{\alpha_{s}\left(M_{H}\right)}{\pi}+\left(35.94-1.36 N_{F}\right)\left(\frac{\alpha_{s}\left(M_{H}\right)}{\pi}\right)^{2}+\left(164.14-25.77 N_{F}+0.259 N_{F}^{2}\right)\left(\frac{\alpha_{s}\left(M_{H}\right)}{\pi}\right)^{3} \\
& +\left(39.34-220.9 N_{F}+9.685 N_{F}^{2}-0.0205 N_{F}^{3}\right)\left(\frac{\alpha_{s}\left(M_{H}\right)}{\pi}\right)^{4} \\
\delta_{t}= & \left(\frac{\alpha_{s}\left(M_{H}\right)}{\pi}\right)^{2}\left[1.57-\frac{2}{3} \log \frac{M_{H}^{2}}{M_{t}^{2}}+\frac{1}{9} \log ^{2} \frac{\bar{m}_{Q}^{2}\left(M_{H}\right)}{M_{H}^{2}}\right]
\end{aligned}
$$

with the number $N_{F}$ of contributing quark flavours. The running quark mass and the QCD coupling are defined at the scale of the Higgs mass, absorbing in this way large mass logarithms. The quark

\footnotetext{
${ }^{1}$ Running-mass effects with respect to QED corrections are not taken into account.
} 
mass effects beyond the Yukawa coupling can be neglected in general, except for heavy quark decays in the threshold region 2. The QCD corrections in this case are given, in terms of the quark pole mass $M_{Q}$, by 42

$$
\Gamma[H \rightarrow Q \bar{Q}]=\frac{3 G_{F} M_{H}}{4 \sqrt{2} \pi} M_{Q}^{2} \beta^{3}\left[1+\frac{4}{3} \frac{\alpha_{s}}{\pi} \delta^{H}\right]
$$

where $\beta=\left(1-4 M_{Q}^{2} / M_{H}^{2}\right)^{1 / 2}$ denotes the velocity of the heavy quarks $Q$. The NLO QCD correction factor reads 42

$$
\delta^{H}=\frac{1}{\beta} A(\beta)+\frac{1}{16 \beta^{3}}\left(3+34 \beta^{2}-13 \beta^{4}\right) \log \frac{1+\beta}{1-\beta}+\frac{3}{8 \beta^{2}}\left(7 \beta^{2}-1\right)
$$

with the function

$$
\begin{aligned}
A(\beta)= & \left(1+\beta^{2}\right)\left[4 \operatorname{Li}_{2}\left(\frac{1-\beta}{1+\beta}\right)+2 \operatorname{Li}_{2}\left(-\frac{1-\beta}{1+\beta}\right)-3 \log \frac{1+\beta}{1-\beta} \log \frac{2}{1+\beta}\right. \\
& \left.-2 \log \frac{1+\beta}{1-\beta} \log \beta\right]-3 \beta \log \frac{4}{1-\beta^{2}}-4 \beta \log \beta
\end{aligned}
$$

$\left(\mathrm{Li}_{2}\right.$ denotes the Spence function, $\mathrm{Li}_{2}(x)=-\int_{0}^{x} d y y^{-1} \log (1-y)$.)

Electroweak corrections to heavy quark and lepton decays are well under control [45, 46]. In the intermediate mass range they can be approximated by [47]

$$
\delta_{\text {elw }}=\frac{3}{2} \frac{\alpha}{\pi} e_{f}^{2}\left(\frac{3}{2}-\log \frac{M_{H}^{2}}{M_{f}^{2}}\right)+\frac{G_{F}}{8 \pi^{2} \sqrt{2}}\left\{k_{f} M_{t}^{2}+M_{W}^{2}\left[-5+\frac{3}{s_{W}^{2}} \log c_{W}^{2}\right]-M_{Z}^{2} \frac{6 v_{f}^{2}-a_{f}^{2}}{2}\right\}
$$

with $v_{f}=2 I_{3 f}-4 e_{f} s_{W}^{2}$ and $a_{f}=2 I_{3 f} . I_{3 f}$ denotes the third component of the electroweak isospin, $e_{f}$ the electric charge of the fermion $f$ and $s_{W}=\sin \theta_{W}$ the sine of the Weinberg angle; $\alpha$ denotes the QED coupling, $M_{t}$ the top quark mass and $M_{W, Z}$ the $W, Z$ boson masses. The large logarithm $\log M_{H}^{2} / M_{f}^{2}$ can be absorbed in the running fermion mass analogous to the QCD corrections. The coefficient $k_{f}$ is equal to 7 for decays into leptons and light quarks; for $b$ quarks it is reduced to 1 due to additional contributions involving top quarks inside the vertex corrections. Within this approximation the twoand three-loop QCD corrections to the $k_{f}$ terms have been computed by means of low-energy theorems [48, 49]. The results imply the simple replacements at NNLO

$$
\begin{aligned}
& k_{f} \rightarrow k_{f} \times\left\{1-\frac{1}{7}\left(\frac{3}{2}+\zeta_{2}\right) \frac{\alpha_{s}\left(M_{t}\right)}{\pi}\right\} \quad \text { for } f \neq b \\
& k_{b} \rightarrow k_{b} \times\left\{1-4\left(1+\zeta_{2}\right) \frac{\alpha_{s}\left(M_{t}\right)}{\pi}\right\}
\end{aligned}
$$

with $\zeta_{2}=\pi^{2} / 6$. The three-loop QCD corrections to the $k_{f}$ term can be found in [49]. Recently, the full mixed QCD-electroweak corrections $\delta_{\text {mixed }}$ have been determined [50]. These turn out to be in the per-mille range if included in the factorized expression for QCD and electroweak corrections as shown in Eq. (14). The electroweak corrections in total are small, of $\mathcal{O}(5 \%)$, in the intermediate mass range. The residual theoretical uncertainties in the intermediate mass range have been estimated as $0.2 \%$ for the QCD part and 0.5\% for electroweak corrections including mixed contributions in the factorized approach [51].

\footnotetext{
${ }^{2}$ The inclusion of the full quark mass effects is motivated by a proper treatment of off-shell Higgs splittings into quark pairs and by determining all subleading quark-mass effects up to NLO. Moreover, the heavy scalar MSSM Higgs boson can acquire a mass close to the location of the top-antitop threshold.
} 


\subsubsection{Minimal supersymmetric extension}

At lowest order the leptonic decay width of neutral MSSM Higgs boson 3 decays is given by [40, 41]

$$
\Gamma\left[\Phi \rightarrow l^{+} l^{-}\right]=\frac{G_{F} M_{\Phi}}{4 \sqrt{2} \pi}\left(g_{l}^{\Phi}\right)^{2} m_{l}^{2} \beta^{p}
$$

where $g_{l}^{\Phi}$ denotes the corresponding MSSM coupling factor, presented in Table 1, $\beta=\left(1-4 m_{l}^{2} / M_{\Phi}^{2}\right)^{1 / 2}$ the velocity of the final-state leptons and $p=3(1)$ the exponent for scalar (pseudoscalar) Higgs particles. The $\tau$ pair decays play a significant role, with a branching ratio of up to about $10 \%$. Muon decays can develop branching ratios of a few $10^{-4}$. All other leptonic decay modes are phenomenologically irrelevant.

The analogous leptonic decay width of the charged Higgs boson reads

$$
\Gamma\left[H^{+} \rightarrow \nu \bar{l}\right]=\frac{G_{F} M_{H^{ \pm}}}{4 \sqrt{2} \pi} m_{l}^{2} \operatorname{tg}^{2} \beta\left(1-\frac{m_{l}^{2}}{M_{H^{ \pm}}^{2}}\right)^{3}
$$

The decay mode into $\tau^{+} \nu_{\tau}$ reaches branching ratios of more than $90 \%$ below the $t \bar{b}$ threshold and the muonic one ranges at a few $10^{-4}$. All other leptonic decay channels of the charged Higgs bosons are unimportant.

For large Higgs masses $\left[M_{\Phi}^{2} \gg M_{Q}^{2}\right]$ the QCD-corrected decay widths of the MSSM Higgs particles into quarks can be obtained from evaluating the analogous diagrams as presented in Fig. 2, where the SM Higgs particle $H$ has to be substituted by the corresponding MSSM Higgs boson $\Phi$ [42, 44]:

$$
\Gamma[\Phi \rightarrow Q \bar{Q}]=\frac{3 G_{F} M_{\Phi}}{4 \sqrt{2} \pi} \bar{m}_{Q}^{2}\left(M_{\Phi}\right)\left(g_{Q}^{\Phi}\right)^{2}\left[1+\delta_{\mathrm{QCD}}+\delta_{t}^{\Phi}\right]
$$

Neglecting regular quark mass effects, the QCD corrections $\delta_{\mathrm{QCD}}$ are presented in Eq. (15) and the top quark induced contributions read as [44]

$$
\begin{aligned}
\delta_{t}^{h / H} & =\frac{g_{t}^{h / H}}{g_{Q}^{h / H}}\left(\frac{\alpha_{s}\left(M_{h / H}\right)}{\pi}\right)^{2}\left[1.57-\frac{2}{3} \log \frac{M_{h / H}^{2}}{M_{t}^{2}}+\frac{1}{9} \log ^{2} \frac{\bar{m}_{Q}^{2}\left(M_{h / H}\right)}{M_{h / H}^{2}}\right] \\
\delta_{t}^{A} & =\frac{g_{t}^{A}}{g_{Q}^{A}}\left(\frac{\alpha_{s}\left(M_{A}\right)}{\pi}\right)^{2}\left[3.83-\log \frac{M_{A}^{2}}{M_{t}^{2}}+\frac{1}{6} \log ^{2} \frac{\bar{m}_{Q}^{2}\left(M_{A}\right)}{M_{A}^{2}}\right]
\end{aligned}
$$

Analogous to the SM case the large logarithmic contributions of the QCD corrections are absorbed in the running $\overline{\mathrm{MS}}$ quark mass $\bar{m}_{Q}\left(M_{\Phi}\right)$ at the scale of the corresponding Higgs mass $M_{\Phi}$.

The quark decay width of the charged Higgs boson reads, in the large Higgs mass regime $M_{H^{ \pm}} \gg$ $M_{U}+M_{D}$, as $[52,53]$

$$
\Gamma\left[H^{+} \rightarrow U \bar{D}\right]=\frac{3 G_{F} M_{H^{ \pm}}}{4 \sqrt{2} \pi}\left|V_{U D}\right|^{2}\left[\bar{m}_{U}^{2}\left(M_{H^{ \pm}}\right)\left(g_{U}^{A}\right)^{2}+\bar{m}_{D}^{2}\left(M_{H^{ \pm}}\right)\left(g_{D}^{A}\right)^{2}\right]\left(1+\delta_{\mathrm{QCD}}\right)
$$

(Eq. (24) is valid if either the first or the second term is dominant.) where $U(D)$ denote heavy up(down)type quarks. The relative couplings $g_{Q}^{A}$ have been collected in Table 1 and the coefficient $V_{U D}$ denotes the CKM matrix element of the transition of $D$ to $U$ quarks. The QCD correction factor $\delta_{\mathrm{QCD}}$ is given in Eq. (15), where large logarithmic terms are again absorbed in the running $\overline{\mathrm{MS}}$ masses $\bar{m}_{U, D}\left(M_{H^{ \pm}}\right)$ at the scale of the charged Higgs mass $M_{H^{ \pm}}$. In the threshold regions mass effects play a significant

\footnotetext{
${ }^{3}$ In the following we denote the different types of neutral Higgs particles collectively by $\Phi=h, H, A$.
} 
role. The partial decay widths of the neutral Higgs bosons $\Phi=h, H$ and $A$ into heavy quark pairs, in terms of the quark pole mass $M_{Q}$, can be cast into the form [42]

$$
\Gamma[\Phi \rightarrow Q \bar{Q}]=\frac{3 G_{F} M_{\Phi}}{4 \sqrt{2} \pi}\left(g_{Q}^{\Phi}\right)^{2} M_{Q}^{2} \beta^{p}\left[1+\frac{4}{3} \frac{\alpha_{s}}{\pi} \delta^{\Phi}\right]
$$

where $\beta=\left(1-4 M_{Q}^{2} / M_{\Phi}^{2}\right)^{1 / 2}$ denotes the velocity of the final-state quarks and $p=3(1)$ the exponent for scalar (pseudoscalar) Higgs bosons. The different powers in $\beta$ are related to the property that the $Q \bar{Q}$ pairs are produced in a $\mathcal{P}$-wave $(\mathcal{S}$-wave) for (pseudo)scalar Higgs decays. To next-to-leading order, the QCD correction factor is given by Eq. (17) for the scalar Higgs particles $h, H$, while for the CP-odd Higgs boson $A$ it reads as 42 .

$$
\delta^{A}=\frac{1}{\beta} A(\beta)+\frac{1}{16 \beta}\left(19+2 \beta^{2}+3 \beta^{4}\right) \log \frac{1+\beta}{1-\beta}+\frac{3}{8}\left(7-\beta^{2}\right)
$$

with the function $A(\beta)$ defined in Eq. (18). The QCD corrections in the $t \bar{t}$ threshold region are moderate, apart from a Coulomb singularity, which is regularized by taking into account the finite top quark decay width.

The partial decay width of the charged Higgs particles into heavy quarks can be expressed as [53]

$$
\begin{aligned}
\Gamma\left[H^{+} \rightarrow U \bar{D}\right]= & \frac{3 G_{F} M_{H^{ \pm}}}{4 \sqrt{2} \pi}\left|V_{U D}\right|^{2} \lambda^{1 / 2}\left\{( 1 - \mu _ { U } - \mu _ { D } ) \left[\frac{M_{U}^{2}}{\operatorname{tg}^{2} \beta}\left(1+\frac{4}{3} \frac{\alpha_{s}}{\pi} \delta_{U D}^{+}\right)\right.\right. \\
& \left.\left.+M_{D}^{2} \operatorname{tg}^{2} \beta\left(1+\frac{4}{3} \frac{\alpha_{s}}{\pi} \delta_{D U}^{+}\right)\right]-4 M_{U} M_{D} \sqrt{\mu_{U} \mu_{D}}\left(1+\frac{4}{3} \frac{\alpha_{s}}{\pi} \delta_{U D}^{-}\right)\right\}
\end{aligned}
$$

where $\mu_{i}=M_{i}^{2} / M_{H^{ \pm}}^{2}$, and $\lambda=\left(1-\mu_{U}-\mu_{D}\right)^{2}-4 \mu_{U} \mu_{D}$ denotes the usual two-body phase-space function. The quark masses $M_{U, D}$ are the pole masses. The QCD factors $\delta_{i j}^{ \pm}(i, j=U, D)$ are given by 53

$$
\begin{aligned}
& \delta_{i j}^{+}=\frac{9}{4}+\frac{3-2 \mu_{i}+2 \mu_{j}}{4} \log \frac{\mu_{i}}{\mu_{j}}+\frac{\left(\frac{3}{2}-\mu_{i}-\mu_{j}\right) \lambda+5 \mu_{i} \mu_{j}}{2 \lambda^{1 / 2}\left(1-\mu_{i}-\mu_{j}\right)} \log x_{i} x_{j}+B_{i j} \\
& \delta_{i j}^{-}=3+\frac{\mu_{j}-\mu_{i}}{2} \log \frac{\mu_{i}}{\mu_{j}}+\frac{\lambda+2\left(1-\mu_{i}-\mu_{j}\right)}{2 \lambda^{1 / 2}} \log x_{i} x_{j}+B_{i j}
\end{aligned}
$$

with the scaling variables $x_{i}=2 \mu_{i} /\left[1-\mu_{i}-\mu_{j}+\lambda^{1 / 2}\right]$ and the generic function

$$
\begin{aligned}
B_{i j}= & \frac{1-\mu_{i}-\mu_{j}}{\lambda^{1 / 2}}\left[4 \operatorname{Li}_{2}\left(x_{i} x_{j}\right)-2 \operatorname{Li}_{2}\left(-x_{i}\right)-2 \operatorname{Li}_{2}\left(-x_{j}\right)+2 \log x_{i} x_{j} \log \left(1-x_{i} x_{j}\right)\right. \\
& -4\left[\log x_{i} \log \left(1+x_{i}\right)-\log x_{j} \log \left(1+x_{j}\right)\right] \\
& +\frac{\left.\left.\lambda^{1 / 2}+\mu_{i}-\mu_{j}\right)+\frac{x_{i} x_{j}}{1-x_{i} x_{j}} \log x_{i} x_{j}\right]}{\lambda^{1 / 2}}\left[\log \left(1+x_{i}\right)-\frac{x_{i}}{1+x_{i}} \log x_{i}\right] \\
& +\frac{\lambda^{1 / 2}-\mu_{i}+\mu_{j}}{\lambda^{1 / 2}}\left[\log \left(1+x_{j}\right)-\frac{x_{j}}{1+x_{j}} \log x_{j}\right]
\end{aligned}
$$

The transition from the threshold region, involving mass effects, to the renormalization-group-improved large Higgs mass regime is provided by a smooth linear interpolation analogous to the SM case in all heavy quark decay modes in the program HDECAY [54].

The full SUSY-electroweak and SUSY-QCD corrections to the fermionic decay modes have been computed [36, 37, 55]. They turn out to be moderate for small values of $\operatorname{tg} \beta$, of the order of about $10 \%$. 
Only for large values of $\operatorname{tg} \beta>10$ do the gluino corrections induce large corrections due to the $\Delta_{f}$ terms of down-type fermions as discussed in the introduction. These effects are in particular relevant for the MSSM Higgs boson decays into bottom quarks. The final NLO decay width into bottom quarks can be written as

$$
\Gamma[\Phi \rightarrow b \bar{b}]=\frac{3 G_{F} M_{\Phi}}{4 \sqrt{2} \pi} \bar{m}_{b}^{2}\left(M_{\Phi}\right) \tilde{g}_{b}^{\Phi}\left[1+\delta_{\mathrm{QCD}}+\delta_{t}^{\Phi}\right]\left[\tilde{g}_{b}^{\Phi}+g_{b}^{\Phi} \delta_{S Q C D}^{r e m}\right]
$$

where the effective couplings $\tilde{g}_{b}^{\Phi}$ are given in Eq. (12) and thus resum the leading $\Delta_{b}$ terms. The contribution $\delta_{S Q C D}^{r e m}$ denotes the remainder of the full SUSY-QCD corrections after absorbing the $\Delta_{b}$ terms in the effective Yukawa couplings. This remainder turns out to be small for all MSSM Higgs bosons even for large Higgs masses [37].

Below the $t \bar{t}$ threshold, heavy neutral Higgs boson decays into off-shell top quarks are sizeable, thus modifying the profile of these Higgs particles significantly in this region. The dominant below-threshold contributions can be obtained from the SM expression for the scalar Higgs bosons [56]

$$
\frac{d \Gamma}{d x_{1} d x_{2}}\left(\phi \rightarrow t t^{*} \rightarrow W t b\right)=\left(g_{t}^{\phi}\right)^{2} \frac{3 G_{F}^{2}}{32 \pi^{3}} M_{t}^{2} M_{\phi}^{3} \frac{\Gamma_{0}^{\phi}}{y_{1}^{2}+\gamma_{t} \kappa_{t}}
$$

with the reduced energies $x_{1,2}=2 E_{t, b} / M_{\phi}(\phi=h, H, A)$, the scaling variables $y_{1,2}=1-x_{1,2}, \kappa_{i}=$ $M_{i}^{2} / M_{\phi}^{2}$ and the reduced decay widths of the virtual particles $\gamma_{i}=\Gamma_{i}^{2} / M_{\phi}^{2}$. The squared amplitudes can be written as [56]

$$
\begin{aligned}
\Gamma_{0}^{H}= & y_{1}^{2}\left(1-y_{1}-y_{2}+\kappa_{W}-5 \kappa_{t}\right)+2 \kappa_{W}\left(y_{1} y_{2}-\kappa_{W}-2 \kappa_{t} y_{1}+4 \kappa_{t} \kappa_{W}\right) \\
& -\kappa_{t} y_{1} y_{2}+\kappa_{t}\left(1-4 \kappa_{t}\right)\left(2 y_{1}+\kappa_{W}+\kappa_{t}\right) \\
\Gamma_{0}^{A}= & y_{1}^{2}\left(1-y_{1}-y_{2}+\kappa_{W}-\kappa_{t}\right)+2 \kappa_{W}\left(y_{1} y_{2}-\kappa_{W}\right)-\kappa_{t}\left(y_{1} y_{2}-2 y_{1}-\kappa_{W}-\kappa_{t}\right)
\end{aligned}
$$

The differential decays width of Eq. (30) has to be integrated over the $x_{1}, x_{2}$ region, bounded by

$$
\left|\frac{2\left(1-x_{1}-x_{2}+\kappa_{t}+\kappa_{b}-\kappa_{W}\right)+x_{1} x_{2}}{\sqrt{x_{1}^{2}-4 \kappa_{t}} \sqrt{x_{2}^{2}-4 \kappa_{b}}}\right| \leq 1
$$

In these formulae $W$ - and charged-Higgs-boson exchange contributions are neglected, because they are suppressed with respect to the off-shell top quark contribution to $W t b$ final states. However, for the sake of completeness they are included in HDECAY [54]. Their explicit expressions can be found in [56]. The transition from below to above the threshold is provided by a smooth cubic interpolation. Below-threshold decays yield a $t \bar{t}$ branching ratio at the per-cent level already for heavy scalar and pseudoscalar Higgs masses $M_{H, A} \sim 300 \mathrm{GeV}$.

Below the $t \bar{b}$ threshold off-shell charged-Higgs decays $H^{+} \rightarrow t^{*} \bar{b} \rightarrow b \bar{b} W^{+}$are important. For $M_{H^{ \pm}}<M_{t}+M_{b}-\Gamma_{t}$, where $\Gamma_{t}$ denotes the top width, their expression can be cast into the form [56]

$$
\begin{aligned}
\Gamma\left(H^{+} \rightarrow t^{*} \bar{b} \rightarrow W b \bar{b}\right)= & \frac{3 G_{F}^{2} M_{t}^{4}}{64 \pi^{3} \operatorname{tg}^{2} \beta} M_{H^{ \pm}}\left\{\frac{\kappa_{W}^{2}}{\kappa_{t}^{3}}\left(4 \kappa_{W} \kappa_{t}+3 \kappa_{t}-4 \kappa_{W}\right) \log \frac{\kappa_{W}\left(\kappa_{t}-1\right)}{\kappa_{t}-\kappa_{W}}\right. \\
& +\left(3 \kappa_{t}^{2}-4 \kappa_{t}-3 \kappa_{W}^{2}+1\right) \log \frac{\kappa_{t}-1}{\kappa_{t}-\kappa_{W}}-\frac{5}{2} \\
& \left.+\frac{1-\kappa_{W}}{\kappa_{t}^{2}}\left(3 \kappa_{t}^{3}-\kappa_{t} \kappa_{W}-2 \kappa_{t} \kappa_{W}^{2}+4 \kappa_{W}^{2}\right)+\kappa_{W}\left(4-\frac{3}{2} \kappa_{W}\right)\right\}
\end{aligned}
$$

with the scaling variables $\kappa_{i}=M_{i}^{2} / M_{H^{ \pm}}^{2}(i=t, W)$. The bottom mass has been neglected in Eq. (33), but it is taken into account in HDECAY by performing a numerical integration of the corresponding Dalitz plot density, given in [56]. The off-shell branching ratio can reach the per-cent level for charged Higgs masses above about $100 \mathrm{GeV}$ for small $\operatorname{tg} \beta$, which is significantly below the $t \bar{b}$ threshold $M_{H^{ \pm}} \sim 180$ $\mathrm{GeV}$. 


\subsection{Higgs boson decays into intermediate gauge bosons}

\subsubsection{Standard Model}

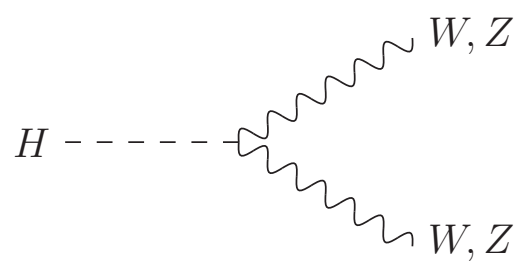

Figure 3: Diagram contributing to $H \rightarrow V V[V=W, Z]$.

Above the $W W$ and $Z Z$ decay thresholds, the partial decay widths into on-shell pairs of massive gauge bosons $(V=W, Z)$ at lowest order (see Fig. 3) are given by [7]

$$
\Gamma(H \rightarrow V V)=\delta_{V} \frac{G_{F} M_{H}^{3}}{16 \sqrt{2} \pi} \beta\left(1-4 x+12 x^{2}\right)
$$

with the abbreviations $x=M_{V}^{2} / M_{H}^{2}, \beta=\sqrt{1-4 x}$ and $\delta_{V}=2(1)$ for $V=W(Z)$.

The NLO electroweak corrections are small and amount to less than about $5 \%$ in the intermediate mass range [45, 57]. Furthermore the QCD corrections to the leading top mass corrections of $\mathcal{O}\left(G_{F} M_{t}^{2}\right)$ have been calculated up to $\mathrm{N}^{3} \mathrm{LO}$. At $\mathrm{NNLO}$ they rescale the $W W, Z Z$ decay widths by [58, 59]

$$
\begin{aligned}
\Gamma(H \rightarrow Z Z) & =\Gamma_{L O}(H \rightarrow Z Z)\left\{1-x_{t}\left[5-\left(15-2 \zeta_{2}\right) \frac{\alpha_{s}}{\pi}\right]\right\} \\
\Gamma(H \rightarrow W W) & =\Gamma_{L O}(H \rightarrow W W)\left\{1-x_{t}\left[5-\left(9-2 \zeta_{2}\right) \frac{\alpha_{s}}{\pi}\right]\right\}
\end{aligned}
$$

with $x_{t}=G_{F} M_{t}^{2} /\left(8 \sqrt{2} \pi^{2}\right)$. The three-loop corrections can be found in [60].

Below threshold the decays into off-shell gauge particles are important [61, 62]. For Higgs masses slightly larger than the corresponding gauge boson mass the decay widths into pairs of off-shell gauge bosons can be cast into the form 62 ]

$$
\Gamma\left(H \rightarrow V^{*} V^{*}\right)=\frac{1}{\pi^{2}} \int_{0}^{M_{H}^{2}} \frac{d Q_{1}^{2} M_{V} \Gamma_{V}}{\left(Q_{1}^{2}-M_{V}^{2}\right)^{2}+M_{V}^{2} \Gamma_{V}^{2}} \int_{0}^{\left(M_{H}-Q_{1}\right)^{2}} \frac{d Q_{2}^{2} M_{V} \Gamma_{V}}{\left(Q_{2}^{2}-M_{V}^{2}\right)^{2}+M_{V}^{2} \Gamma_{V}^{2}} \Gamma_{0}
$$

with $Q_{1}^{2}, Q_{2}^{2}$ being the squared invariant masses of the virtual gauge bosons, $M_{V}$ and $\Gamma_{V}$ their masses and total decay widths; $\Gamma_{0}$ is given by

$$
\Gamma_{0}=\delta_{V} \frac{G_{F} M_{H}^{3}}{16 \sqrt{2} \pi} \sqrt{\lambda\left(Q_{1}^{2}, Q_{2}^{2} ; M_{H}^{2}\right)}\left[\lambda\left(Q_{1}^{2}, Q_{2}^{2} ; M_{H}^{2}\right)+12 \frac{Q_{1}^{2} Q_{2}^{2}}{M_{H}^{4}}\right]
$$

with the phase-space factor

$$
\lambda(x, y ; z)=\left(1-\frac{x}{z}-\frac{y}{z}\right)^{2}-4 \frac{x y}{z^{2}} .
$$

The branching ratios of double off-shell decays reach the per cent level for Higgs masses above about 100 (110) GeV for off-shell $W(Z)$ boson pairs. Off-shell effects modify the partial decay widths by

\footnotetext{
${ }^{4}$ Even though the SM Higgs mass is too small these expressions play a role for off-shell Higgs bosons splitting into on-shell $W$ - or $Z$-boson pairs.
} 
$\mathcal{O}(10 \%)$ above the $W W$ and $Z Z$ thresholds. During the last years the complete electroweak corrections have been obtained for the full Higgs boson decays into four fermions that include the resonant and non-resonant contributions to Higgs boson decays into intermediate gauge bosons $W, Z$ [63]. In Eq. (38) they can be approximated by the improved Born approximation [63],

$H \rightarrow Z Z \rightarrow 4 f:$

$$
\begin{aligned}
\Gamma_{0} \rightarrow \Gamma_{0} \times \Re e & \left\{1+\frac{G_{F} \mu_{t}^{2}}{8 \sqrt{2} \pi^{2}}\left[1-\frac{6 \cos \theta_{W}}{\sin \theta_{W}}\left(\frac{e_{f_{1}}}{g_{Z f_{1} f_{1}}^{\sigma_{1}}}+\frac{e_{f_{3}}}{g_{Z f_{3} f_{3}}^{\sigma_{3}}}\right)+\tau_{H Z Z}\left(\frac{M_{H}^{2}}{\mu_{t}^{2}}\right)\right]\right. \\
+ & \frac{G_{F} M_{H}^{2}}{8 \sqrt{2} \pi^{2}}\left(\frac{5 \pi^{2}}{6}-3 \sqrt{3} \pi+\frac{19}{2}\right)+62.0308(86)\left(\frac{G_{F} M_{H}^{2}}{16 \pi^{2} \sqrt{2}}\right)^{2} \\
+ & \left.\delta_{Z \rightarrow f_{1} \bar{f}_{2}}^{Q C D}+\delta_{Z \rightarrow f_{3} \bar{f}_{4}}^{Q C D}+c_{H Z Z}\right\},
\end{aligned}
$$

$\underline{H \rightarrow W W \rightarrow 4 f:}$

$$
\begin{aligned}
\Gamma_{0} \rightarrow \Gamma_{0} \times \Re e & \left\{1+\frac{G_{F} \mu_{t}^{2}}{8 \pi^{2} \sqrt{2}}\left[-5+\tau_{H W W}\left(\frac{M_{H}^{2}}{\mu_{t}^{2}}\right)\right]\right. \\
+ & \frac{G_{F} M_{H}^{2}}{8 \sqrt{2} \pi^{2}}\left(\frac{5 \pi^{2}}{6}-3 \sqrt{3} \pi+\frac{19}{2}\right)+62.0308(86)\left(\frac{G_{F} M_{H}^{2}}{16 \pi^{2} \sqrt{2}}\right)^{2} \\
+ & \left.g(\bar{\beta}) \delta_{\text {Coul }}\left(M_{H}^{2}, Q_{1}^{2}, Q_{2}^{2}\right)+\delta_{W \rightarrow f_{1} \bar{f}_{2}}^{Q C D}+\delta_{W \rightarrow f_{3} \bar{f}_{4}}^{Q C D}+c_{H W W}\right\}
\end{aligned}
$$

with the electric charges $e_{f}$ of the fermions and the fermionic couplings to the $Z$ boson

$$
g_{Z f f}^{+}=-\frac{\sin \theta_{W}}{\cos \theta_{W}} e_{f}, \quad g_{Z f f}^{-}=-\frac{\sin \theta_{W}}{\cos \theta_{W}} e_{f}+\frac{I_{3 f}}{\cos \theta_{W} \sin \theta_{W}}
$$

and the auxiliary functions

$$
\begin{gathered}
\tau_{H Z Z}\left(\frac{M_{H}^{2}}{\mu_{t}^{2}}\right)=20+6 \beta_{t}^{2}+3 \beta_{t}\left(\beta_{t}^{2}+1\right) \log x_{t}+3\left(1-\beta_{t}^{2}\right) \log ^{2} x_{t}, \\
\tau_{H W W}\left(\frac{M_{H}^{2}}{\mu_{t}^{2}}\right)=8+12 \beta_{t}^{2}+3 \beta_{t}\left(3 \beta_{t}^{2}-1\right) \log x_{t}+\frac{3}{2}\left(1-\beta_{t}^{2}\right)^{2} \log ^{2} x_{t},
\end{gathered}
$$

where

$$
\beta_{t}=\sqrt{1-\frac{4 \mu_{t}^{2}}{M_{H}^{2}}}, \quad x_{t}=\frac{\beta_{t}-1}{\beta_{t}+1}
$$

and $\mu_{t}$ denotes the complex top mass, given by $\mu_{t}^{2}=m_{t}^{2}-i m_{t} \Gamma_{t}$ with the top width $\Gamma_{t}$. The factor $\delta_{\text {Coul }}$ describes the Coulomb singularity,

$$
\begin{aligned}
\delta_{\text {Coul }}\left(s, Q_{1}^{2}, Q_{2}^{2}\right) & =\frac{\alpha(0)}{\bar{\beta}} \Im m\left\{\log \left(\frac{\beta-\bar{\beta}+\Delta_{M}}{\beta+\bar{\beta}+\Delta_{M}}\right)\right\} \\
\bar{\beta} & =\frac{\sqrt{s^{2}+Q_{1}^{4}+Q_{2}^{4}-2 s Q_{1}^{2}-2 s Q_{2}^{2}-2 Q_{1}^{2} Q_{2}^{2}}}{s} \\
\beta & =\sqrt{1-\frac{4 \mu_{W}^{2}}{s}}, \quad \Delta_{M}=\frac{\left|Q_{1}^{2}-Q_{2}^{2}\right|}{s},
\end{aligned}
$$


where $\alpha(0)$ denotes the fine-structure constant in the Thomson limit and the complex $W$ mass $\mu_{W}^{2}=$ $M_{W}^{2}-i M_{W} \Gamma_{W}$ is given in terms of the $W$ pole mass $M_{W}$ and the $W$ decay width $\Gamma_{W}$. The function $g(\bar{\beta})$ reads

$$
g(\bar{\beta})=\left(1-\bar{\beta}^{2}\right)^{2}
$$

Finally the QCD correction factors to the gauge-boson decays are given by

$$
\delta_{V \rightarrow l_{i} \bar{l}_{j}}^{Q C D}=0, \quad \delta_{V \rightarrow q_{i} \bar{q}_{j}}^{Q C D}=\frac{\alpha_{s}}{\pi}
$$

and the continuum contributions can be fitted as

$$
c_{H Z Z}=3 \%, \quad c_{H W W}=4 \%
$$

They constitute auxiliary corrections beyond the previous terms to compensate the remaining offset between the exact corrections and the improved Born approximation [63. In general there are additional non-resonant LO contributions beyond those of Eq. (37). However, they turn out to range at the percent level [63]. Recently the full electroweak NLO expressions to the leptonic Higgs decays $H \rightarrow 4 \ell$ have been matched to parton showers [64], indicating that there are a few exclusive observables where multiple photon effects may be relevant. The theoretical uncertainties due to missing corrections beyond NLO have been estimated as $0.5 \%$ in the intermediate mass range for the inclusive decay rate into four fermions [65, 66].

\subsubsection{Minimal supersymmetric extension}

The dominant part of the partial widths of the scalar MSSM Higgs bosons into $W$ and $Z$ boson pairs can be obtained from the SM Higgs decay widths by rescaling with the corresponding MSSM couplings $g_{V}^{h / H}$ as listed in Table 1:

$$
\Gamma\left(h / H \rightarrow V^{(*)} V^{(*)}\right)=\left(g_{V}^{h / H}\right)^{2} \Gamma\left(H_{S M} \rightarrow V^{(*)} V^{(*)}\right)
$$

Their branching ratios are strongly reduced by reduced MSSM couplings and additional open leading decay modes, and thus do not play a dominant role as in the SM case. Nevertheless the $W W, Z Z$ branching ratios can reach values of $\mathcal{O}(10 \%)$ for the heavy scalar Higgs boson $H$ for small $\operatorname{tg} \beta$. Off-shell $W W, Z Z$ decays can pick up several per-cent of the light scalar Higgs decays at the upper end of its mass range. The pseudoscalar Higgs particle does not couple to $W$ and $Z$ bosons at tree level. The SUSY-electroweak corrections to scalar Higgs boson decays into off-shell $W W$ and $Z Z$ pairs have been computed [67]. While being similar to the SM-Higgs case they can be enhanced to the 10\%-level in particular MSSM scenarios.

\subsection{Higgs boson decays into gluons}

\subsubsection{Standard Model}

The decay of the Higgs boson into gluons is mediated by heavy quark loops in the SM, with top quarks providing the dominant contribution, see Fig. 4. The partial decay width [40, 68, 69, 70] is at lowest order given by

$$
\Gamma_{L O}[H \rightarrow g g]=\frac{G_{F} \alpha_{s}^{2} M_{H}^{3}}{36 \sqrt{2} \pi^{3}}\left|\sum_{Q} A_{Q}^{H}\left(\tau_{Q}\right)\right|^{2}
$$




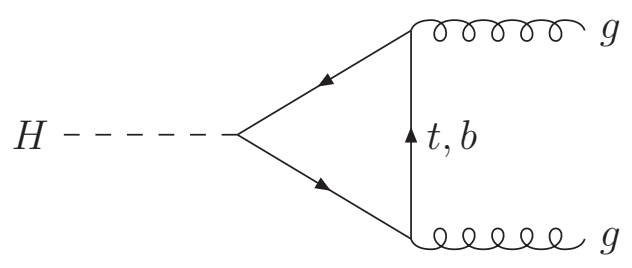

Figure 4: Diagrams contributing to $H \rightarrow g g$ at lowest order.

with the form factor

$$
\begin{aligned}
A_{Q}^{H}(\tau) & =\frac{3}{2} \tau[1+(1-\tau) f(\tau)] \\
f(\tau) & = \begin{cases}\arcsin ^{2} \frac{1}{\sqrt{\tau}} & \tau \geq 1 \\
-\frac{1}{4}\left[\log \frac{1+\sqrt{1-\tau}}{1-\sqrt{1-\tau}}-i \pi\right]^{2} & \tau<1\end{cases}
\end{aligned}
$$

The parameter $\tau_{Q}=4 M_{Q}^{2} / M_{H}^{2}$ is defined by the pole mass $M_{Q}$ of the heavy loop quark $Q$. For large quark masses the form factor approaches unity. The QCD corrections are known up to NLO including the full quark mass dependence [68 and up to $\mathrm{N}^{3} \mathrm{LO}$ in the limit of heavy top quarks [70, 71, 72. For a Higgs mass $M_{H} \sim 125 \mathrm{GeV}$ they can be expressed as

$$
\begin{aligned}
\Gamma[H \rightarrow g g]= & \Gamma_{L O}\left\{1+\left(23.75-1.167 N_{F}+\Delta_{m}\right) \frac{\alpha_{s}\left(M_{H}\right)}{\pi}\right. \\
+ & \left(370.20-47.19 N_{F}+0.902 N_{F}^{2}+\left(2.375+0.667 N_{F}\right) \log \frac{M_{H}^{2}}{m_{t}^{2}}\right)\left(\frac{\alpha_{s}\left(M_{H}\right)}{\pi}\right)^{2} \\
+ & \left(4533.46-1062.82 N_{F}+52.62 N_{F}^{2}-0.5378 N_{F}^{2}+\left(66.66+14.60 N_{F}\right.\right. \\
& \left.\left.\left.-0.6887 N_{F}^{2}\right) \log \frac{M_{H}^{2}}{m_{t}^{2}}+\left(6.53+1.44 N_{F}-0.111 N_{F}^{2}\right) \log ^{2} \frac{M_{H}^{2}}{m_{t}^{2}}\right)\left(\frac{\alpha_{s}\left(M_{H}\right)}{\pi}\right)^{3}\right\} \\
\approx & \Gamma_{L O}\{1+0.67+0.20+0.02\}
\end{aligned}
$$

for $N_{F}=5$ light quark flavours. The term $\Delta_{m} \approx 0.76$ denotes the NLO quark-mass effects from the top, bottom and charm quarks [68]. The radiative corrections turn out to be very large: the decay width is increased by about $90 \%$ in the intermediate mass range. The approximation of the heavy top limit is valid for the partial gluonic decay width within about $5-10 \%$ for the whole Higgs mass range up to 1 $\mathrm{TeV}$, while it is valid at the per-cent level in the intermediate mass range. The reason for the suppressed quark mass dependence of the relative QCD corrections is the dominance of soft and collinear gluon contributions, which do not resolve the Higgs coupling to gluons and thus lead to a simple rescaling factor. The three-loop [71] and four-loop [72] QCD corrections to the gluonic decay width have been evaluated in the limit of a heavy top quark. They contribute a further amount of $\mathcal{O}(20 \%)$ relative to the lowest order result and thus increase the full NLO expression by $\mathcal{O}(10 \%)$. The reduced size of these corrections signals a significant stabilization of the perturbative result and thus a reliable theoretical prediction.

The QCD corrections in the heavy quark limit can also be obtained by means of a low-energy theorem [40, 73]. The starting point is that, for vanishing Higgs momentum $p_{H} \rightarrow 0$, the entire interaction of 
the Higgs particle with $W, Z$ bosons and fermions can be generated by the substitution

$$
M_{i} \rightarrow M_{i} \times\left[1+\frac{H}{v}\right] \quad(i=f, W, Z)
$$

where the Higgs field $H$ acts as a constant complex number. At higher orders this substitution has to be expressed in terms of bare or $\overline{\mathrm{MS}}$-subtracted parameters [58, 68]. In most of the practical cases the external Higgs particles are treated on-shell, so that $p_{H}^{2}=M_{H}^{2}$ and the mathematical limit of vanishing Higgs momentum coincides with the limit of small Higgs masses or large loop-particle masses. In order to calculate the effective Higgs couplings to gluons in the heavy-quark limit one starts from the heavy quark $Q$ contribution to the kinetic gluon operator [74] that is related to the decoupling relation of the strong coupling constant [75, 76],

$$
\begin{aligned}
\mathcal{L}_{g} & =-\frac{1}{4 \zeta_{\alpha_{s}}} \hat{G}^{a \mu \nu} \hat{G}_{\mu \nu}^{a} \\
\alpha_{s}^{\left(N_{F}\right)}\left(\mu_{R}^{2}\right) & =\zeta_{\alpha_{s}} \alpha_{s}^{\left(N_{F}+1\right)}\left(\mu_{R}^{2}\right)
\end{aligned}
$$

where $N_{F}=5$ is the number of light quark flavours. The gluonic field strength tensor $\hat{G}_{\mu \nu}^{a}$ is defined in the $\left(N_{F}+1\right)$-flavour theory, while the superscript of the strong coupling $\alpha_{s}$ defines the number of active flavours taken into account. The inverse of the decoupling factor $\zeta_{\alpha_{s}}$ develops a perturbative expansion that contains a logarithmic top mass dependence, i.e. it can be expressed in terms of the logarithm $L_{t}=\log \left(\mu_{R}^{2} /{\overline{m_{t}}}^{2}\left(\mu_{R}^{2}\right)\right)$, where ${\overline{m_{t}}}^{2}\left(\mu_{R}^{2}\right)$ denotes the $\overline{\mathrm{MS}}$ top mass at the renormalization scale $\mu_{R}$. The substitution of Eq. (52) implies the shift

$$
L_{t} \rightarrow \bar{L}_{t}=L_{t}-2 \log \left(1+\frac{H}{v}\right)
$$

Keeping only Higgs-field dependent terms and transforming the gluonic field strength operator and the strong coupling constant by their $N_{F}$-flavour expressions one arrives at the effective Lagrangian in the heavy top-quark limit [74,

$$
\begin{aligned}
\mathcal{L}_{e f f}=\frac{\alpha_{s}}{12 \pi}\{(1+\delta) & \log \left(1+\frac{H}{v}\right)-\frac{\eta}{2} \log ^{2}\left(1+\frac{H}{v}\right) \\
+ & \left.\frac{\rho}{3} \log ^{3}\left(1+\frac{H}{v}\right)-\frac{\sigma}{4} \log ^{4}\left(1+\frac{H}{v}\right)\right\} G^{a \mu \nu} G_{\mu \nu}^{a}
\end{aligned}
$$

with the QCD corrections up to $\mathrm{N}^{4} \mathrm{LO}$

$$
\begin{aligned}
\delta & =\delta_{1} \frac{\alpha_{s}}{\pi}+\delta_{2}\left(\frac{\alpha_{s}}{\pi}\right)^{2}+\delta_{3}\left(\frac{\alpha_{s}}{\pi}\right)^{3}+\delta_{4}\left(\frac{\alpha_{s}}{\pi}\right)^{4}+\mathcal{O}\left(\alpha_{s}^{5}\right) \\
\eta & =\eta_{2}\left(\frac{\alpha_{s}}{\pi}\right)^{2}+\eta_{3}\left(\frac{\alpha_{s}}{\pi}\right)^{3}+\eta_{4}\left(\frac{\alpha_{s}}{\pi}\right)^{4}+\mathcal{O}\left(\alpha_{s}^{5}\right) \\
\rho & =\rho_{3}\left(\frac{\alpha_{s}}{\pi}\right)^{3}+\rho_{4}\left(\frac{\alpha_{s}}{\pi}\right)^{4}+\mathcal{O}\left(\alpha_{s}^{5}\right) \\
\sigma & =\sigma_{4}\left(\frac{\alpha_{s}}{\pi}\right)^{4}+\mathcal{O}\left(\alpha_{s}^{5}\right)
\end{aligned}
$$

The perturbative coefficients are explicitly given by

$$
\delta_{1}=\frac{11}{4}
$$

$$
\delta_{2}=\frac{2777}{288}+\frac{19}{16} L_{t}+N_{F}\left(\frac{L_{t}}{3}-\frac{67}{96}\right)
$$




$$
\begin{aligned}
\delta_{3}= & \frac{897943}{9216} \zeta_{3}-\frac{2892659}{41472}+\frac{209}{64} L_{t}^{2}+\frac{1733}{288} L_{t} \\
+ & N_{F}\left(\frac{40291}{20736}-\frac{110779}{13824} \zeta_{3}+\frac{23}{32} L_{t}^{2}+\frac{55}{54} L_{t}\right)+N_{F}^{2}\left(-\frac{L_{t}^{2}}{18}+\frac{77}{1728} L_{t}-\frac{6865}{31104}\right) \\
\delta_{4}= & -\frac{121}{1440} N_{F} \log ^{5} 2+\frac{3751}{2880} \log ^{5} 2+\frac{685}{41472} N_{F}^{2} \log ^{4} 2+\frac{11679301}{1741824} N_{F} \log ^{4} 2 \\
- & \frac{93970579}{870912} \log ^{4} 2+\frac{121}{144} N_{F} \zeta_{2} \log ^{3} 2-\frac{3751}{288} \zeta_{2} \log ^{3} 2-\frac{685}{6912} N_{F}^{2} \zeta_{2} \log ^{2} 2 \\
- & \frac{11679301}{290304} N_{F} \zeta_{2} \log ^{2} 2+\frac{93970579}{145152} \zeta_{2} \log ^{2} 2+\frac{2057}{192} N_{F} \zeta_{4} \log 2-\frac{63767}{384} \zeta_{4} \log 2 \\
+ & \frac{685}{1728} N_{F}^{2} a_{4}+\frac{11679301}{72576} N_{F} a_{4}-\frac{93970579}{36288} a_{4}+\frac{121}{12} N_{F} a_{5}-\frac{3751}{24} a_{5}+\frac{211}{1728} N_{F}^{3} \zeta_{3} \\
- & \frac{270407}{1492992} N_{F}^{3}+\frac{4091305}{331776} N_{F}^{2} \zeta_{3}-\frac{576757}{55296} N_{F}^{2} \zeta_{4}-\frac{115}{384} N_{F}^{2} \zeta_{5}-\frac{48073}{27648} N_{F}^{2} \\
- & \frac{151369}{725760} N_{F} X_{0}-\frac{12171659669}{38707200} N_{F} \zeta_{3}+\frac{608462731}{11612160} N_{F} \zeta_{4}+\frac{313489}{6912} N_{F} \zeta_{5} \\
+ & \frac{76094378783}{522547200} N_{F}+\frac{4692439}{1451520} X_{0}+\frac{28121193841}{19353600} \zeta_{3}+\frac{4674213853}{2903040} \zeta_{4}-\frac{807193}{1728} \zeta_{5} \\
- & \frac{854201072999}{522547200}+\left(\frac{481}{5184} N_{F}^{3}+\frac{28297}{9216} N_{F}^{2} \zeta_{3}-\frac{21139}{3456} N_{F}^{2}-\frac{32257}{288} N_{F} \zeta_{3}\right. \\
& \left.+\frac{5160073}{41472} N_{F}+\frac{9364157}{12288} \zeta_{3}-\frac{49187545}{55296}\right) L_{t}+\left(-\frac{77}{6912} N_{F}^{3}-\frac{1267}{13824} N_{F}^{2}+\frac{4139}{2304} N_{F}\right. \\
& \left.+\frac{8401}{384}\right) L_{t}^{2}+\left(\frac{1}{108} N_{F}^{3}-\frac{157}{576} N_{F}^{2}+\frac{275}{192} N_{F}+\frac{2299}{256}\right) L_{t}^{3}
\end{aligned}
$$

and

$$
\begin{aligned}
\eta_{2} & =\frac{35}{24}+\frac{2}{3} N_{F} \\
\eta_{3} & =\frac{1333}{432}+\frac{589}{48} L_{t}+N_{F}\left(\frac{1081}{432}+\frac{191}{72} L_{t}\right)+N_{F}^{2}\left(\frac{77}{864}-\frac{2}{9} L_{t}\right) \\
\eta_{4} & =\frac{481}{2592} N_{F}^{3}+N_{F}^{2}\left(\frac{28297}{4608} \zeta_{3}-\frac{373637}{31104}\right)+N_{F}\left(\frac{429965}{1728}-\frac{2985893}{13824} \zeta_{3}\right) \\
& +\frac{26296585}{18432} \zeta_{3}-\frac{143976701}{82944}+\left(-\frac{77}{1728} N_{F}^{3}-\frac{1421}{3456} N_{F}^{2}+\frac{9073}{1728} N_{F}+\frac{45059}{576}\right) L_{t} \\
& +\left(\frac{N_{F}^{3}}{18}-\frac{455}{288} N_{F}^{2}+\frac{63}{8} N_{F}+\frac{6479}{128}\right) L_{t}^{2} \\
\rho_{3} & =\frac{1697}{144}+\frac{175}{72} N_{F}-\frac{2}{9} N_{F}^{2} \\
\rho_{4} & =\frac{130201}{1728}+\frac{18259}{192} L_{t}+N_{F}\left(\frac{5855}{1728}+\frac{2077}{144} L_{t}\right)-N_{F}^{2}\left(\frac{175}{384}+\frac{439}{144} L_{t}\right) \\
& +N_{F}^{3}\left(\frac{L_{t}}{9}-\frac{77}{1728}\right) \\
\sigma_{4} & =\frac{51383}{864}+\frac{317}{36} N_{F}-\frac{47}{24} N_{F}^{2}+\frac{2}{27} N_{F}^{3}
\end{aligned}
$$

where $G_{\mu \nu}^{a}$ denotes the gluon field strength tensor and $\alpha_{s}$ the strong coupling constant with $N_{F}=5$ active flavours. The constants used in these expressions are defined as $a_{n}=L i_{n}(1 / 2)(n=4,5)$ and [77]

$$
X_{0}=\frac{873}{2} \zeta_{5}-384 a_{5}+\frac{16}{5} \log ^{5} 2-32 \zeta_{2} \log ^{3} 2-318 \zeta_{4} \log 2=1.8088795462 \ldots
$$


This effective Lagrangian describes the multi-Higgs couplings to gluons after integrating out the heavy top quark.

For the full calculation of the heavy quark limit given in Eq. (51) the effective $\mathrm{Hgg}(g)$ couplings have to be inserted into the blobs of the effective diagrams shown in Fig. 5 for the NLO contribution. After evaluating these effective massless one-loop contributions the result coincides with the explicit calculation of the two-loop corrections in the heavy quark limit of Eq. (51). This calculation has been extended to $\mathrm{N}^{3} \mathrm{LO}$ analogously [71, 72].
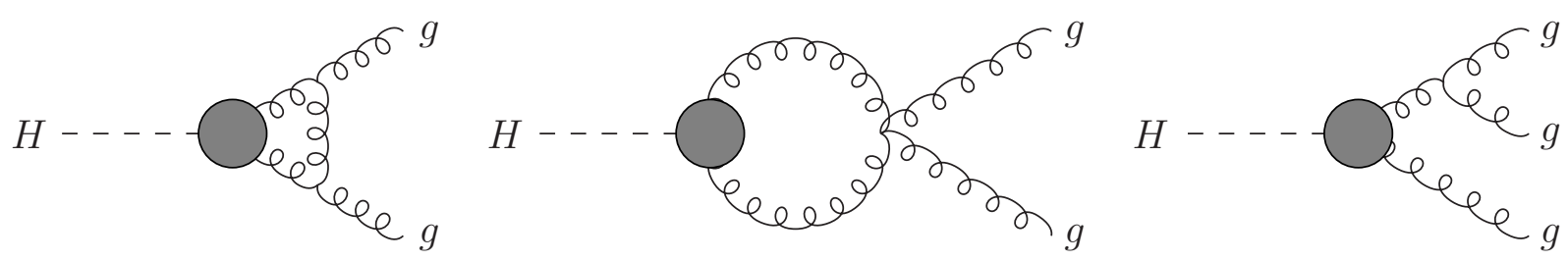

Figure 5: Typical effective diagrams contributing to the NLO QCD corrections to $H \rightarrow g g$ in the heavy quark limit.

Using the discussed low-energy theorem, the electroweak corrections of $\mathcal{O}\left(G_{F} M_{t}^{2}\right)$ to the gluonic decay width, which are mediated by virtual top quarks, can be obtained from the $\mathcal{O}\left(G_{F} M_{t}^{2}\right)$ contribution to the kinetic gluon operator. The final result leads to a simple rescaling of the lowest order decay width [49, 78,

$$
\Gamma(H \rightarrow g g)=\Gamma_{L O}(H \rightarrow g g)\left[1+\frac{G_{F} M_{t}^{2}}{8 \sqrt{2} \pi^{2}}\right]
$$

These corrections enhance the gluonic decay width by about $0.3 \%$ and are thus negligible. These $\mathcal{O}\left(G_{F} M_{t}^{2}\right)$ corrections are part of the full electroweak corrections that have been determined first analytically for the partial light-fermion contributions [79] and finally numerically for the full electroweak corrections [80]. The electroweak corrections increase the partial Higgs decay width into gluons by about 5\%. The residual theoretical uncertainties due to missing electroweak corrections beyond NLO have been estimated as 1\% and due to missing higher-order QCD corrections as 3\% so that the total uncertainty ranges at about 3\% [51, 65].

\subsubsection{Minimal supersymmetric extension}
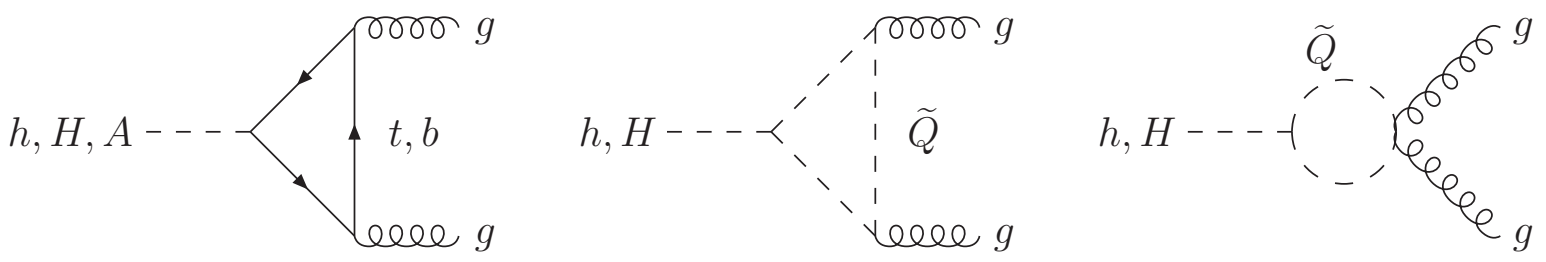

Figure 6: Typical diagrams contributing to $\Phi \rightarrow$ gg at lowest order.

Since the $b$-quark couplings to the Higgs bosons may be strongly enhanced for large $\operatorname{tg} \beta$ and the $t$-quark couplings suppressed in the MSSM, $b$ loops can contribute significantly to the $g g$ coupling so that the 
approximation $M_{Q}^{2} \gg M_{H}^{2}$ can in general no longer be applied. The leading order width for $h, H \rightarrow g g$ is generated by quark and squark loops, the latter contributing significantly for squark masses below about $400 \mathrm{GeV}$ [81]. The contributing diagrams are depicted in Fig. 6. The partial decay widths are given by $68,81,82$

$$
\begin{aligned}
\Gamma_{L O}(h / H \rightarrow g g) & =\frac{G_{F} \alpha_{s}^{2} M_{h / H}^{3}}{36 \sqrt{2} \pi^{3}}\left|\sum_{Q} g_{Q}^{h / H} A_{Q}^{h / H}\left(\tau_{Q}\right)+\sum_{\widetilde{Q}} g_{\widetilde{Q}}^{h / H} A_{\widetilde{Q}}^{h / H}\left(\tau_{\widetilde{Q}}\right)\right|^{2} \\
A_{Q}^{h / H}(\tau) & =\frac{3}{2} \tau[1+(1-\tau) f(\tau)] \\
A_{\widetilde{Q}}^{h / H}(\tau) & =-\frac{3}{4} \tau[1-\tau f(\tau)]
\end{aligned}
$$

with $\tau_{i}=4 M_{i}^{2} / M_{h / H}^{2} \quad(i=Q, \widetilde{Q})$. The function $f(\tau)$ is defined in Eq. (50) and the MSSM couplings $g_{Q}^{h / H}$ can be found in Table 1. The squark couplings $g_{\widetilde{Q}}^{h / H}$ are summarized in Table 2. The amplitudes approach constant values in the limit of large loop particle masses:

$$
\begin{array}{ll}
A_{Q}^{h / H}(\tau) \rightarrow 1 & \text { for } M_{h / H}^{2} \ll 4 M_{Q}^{2} \\
A_{\widetilde{Q}}^{h / H}(\tau) \rightarrow \frac{1}{4} & \text { for } M_{h / H}^{2} \ll 4 M_{\widetilde{Q}}^{2}
\end{array}
$$

The QCD corrections to the quark loops can be adopted from the SM Higgs case as given in Eq. (51) with the Higgs mass $M_{H}$ replaced by the corresponding MSSM Higgs mass $M_{h / H}$. The QCD corrections to the squark loops are known up to NLO including the full mass dependence [83]. Up to NLO the partial decay width is given by

$$
\begin{aligned}
\Gamma[h / H \rightarrow g g] & =\Gamma_{L O}\left\{1+E \frac{\alpha_{s}\left(M_{h / H}\right)}{\pi}\right\} \\
E & \rightarrow \frac{95}{4}-\frac{7}{6} N_{F}+\frac{7}{2} \Re e\left\{\frac{\sum_{\widetilde{Q}} g_{\widetilde{Q}}^{h / H} A_{\widetilde{Q}}^{h / H}\left(\tau_{\widetilde{Q}}\right)}{\sum_{Q} g_{Q}^{h / H} A_{Q}^{h / H}\left(\tau_{Q}\right)}\right\} \quad \text { for } M_{h / H}^{2} \ll 4 M_{Q, \widetilde{Q}}^{2}
\end{aligned}
$$

Analogous to the quark contributions the heavy squark loop correction can be obtained by means of the extension of the previously described low-energy theorem to scalar squark particles [81]. The effective NLO Lagrangian for the squark part is given by [81, 83]

$$
\mathcal{L}_{e f f}=\frac{\alpha_{s}}{48 \pi} G^{a \mu \nu} G_{\mu \nu}^{a} \frac{H}{v}\left[1+\frac{9}{2} \frac{\alpha_{s}}{\pi}\right]
$$

where only non-SUSY-QCD corrections are taken into account, i.e. diagrams with gluino exchange and squark four-point couplings are omitted. Thus the only difference to the quark loops in the heavy loop mass limit arises in the virtual corrections. This leads to the additional last term of Eq. (63).

For the pseudoscalar Higgs decays only quark loops are contributing, and we find [68]

$$
\begin{aligned}
\Gamma_{L O}[A \rightarrow g g] & =\frac{G_{F} \alpha_{s}^{2} M_{A}^{3}}{16 \sqrt{2} \pi^{3}}\left|\sum_{Q} g_{Q}^{A} A_{Q}^{A}\left(\tau_{Q}\right)\right|^{2} \\
A_{Q}^{A}(\tau) & =\tau f(\tau)
\end{aligned}
$$

\footnotetext{
${ }^{5}$ Taking into account the squark four-point couplings for degenerate squark masses, too, the coefficient $9 / 2$ is changed to 29/6 81, 83, 84]. The proper decoupling of gluino contributions in the heavy-mass limit modifies this coefficient to $37 / 6$ for mass-degenerate stop states [85].
} 


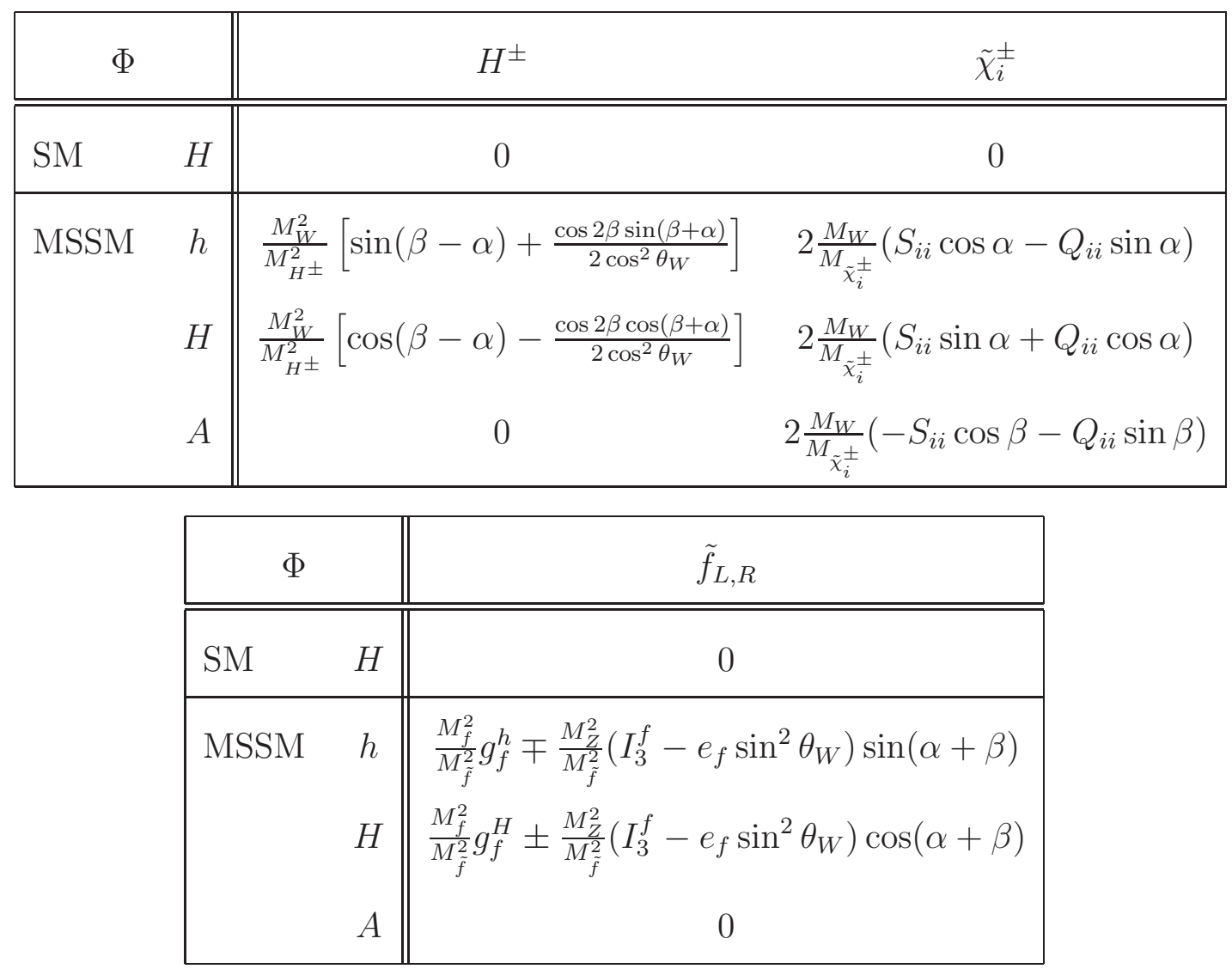

Table 2: MSSM Higgs couplings to charged Higgs bosons, charginos and sfermions relative to the corresponding $S M$ Yukawa couplings. $Q_{i i}$ and $S_{i i}(i=1,2)$ are related to the mixing angles between the charginos $\tilde{\chi}_{1}^{ \pm}$and $\tilde{\chi}_{2}^{ \pm}$, see Refs. [82, 86]. The indices $L, R$ for the sfermion states define the weak charges according to their fermionic superpartners.

with $\tau_{Q}=4 M_{Q}^{2} / M_{A}^{2}$. The MSSM couplings $g_{Q}^{A}$ can be found in Table 1. For large quark masses the quark amplitude approaches unity. In order to get a consistent result for the QCD corrections, the pseudoscalar $\gamma_{5}$ coupling has been regularized in the 't Hooft-Veltman scheme [87] or its extension by Larin [88, which requires an additional finite renormalization of the $A Q \bar{Q}$ vertex [68, 89]. An alternative is provided by the scheme of Ref. [90] that avoids the additional counter terms by giving up the cyclicity of the traces involving Clifford matrices and thus requires a fixed reading point in all diagrams. The heavy quark limit $M_{A}^{2} \ll 4 M_{Q}^{2}$ provides a reasonable approximation in the MSSM parameter range where this decay mode is significant. At the threshold $M_{A}=2 M_{t}$, the QCD corrections develop a Coulomb singularity, which is regularized by including the finite top decay width [91]. The pseudoscalar decay width has been calculated up to NNLO in QCD within the heavy quark limit [92], while the NLO corrections are known exactly [68],

$$
\begin{aligned}
\Gamma[A \rightarrow g g] & =\Gamma_{L O}\left\{1+\left(24.25-1.167 N_{F}+\Delta_{m}\right) \frac{\alpha_{s}\left(M_{A}\right)}{\pi}\right. \\
& \left.+\left(392.22-48.58 N_{F}+0.888 N_{F}^{2}+N_{F} \log \frac{M_{A}^{2}}{m_{t}^{2}}\right)\left(\frac{\alpha_{s}\left(M_{A}\right)}{\pi}\right)^{2}\right\} \\
& \approx \Gamma_{L O}\{1+0.66+0.20\}
\end{aligned}
$$

where the numbers of the last line are for a pseudoscalar mass $M_{A}=200 \mathrm{GeV}$. The top, bottom and charm mass effects amount to $\Delta_{m} \approx 1.3$ for $\operatorname{tg} \beta=1$. For larger values of $\operatorname{tg} \beta$ quark mass effects are 
larger due to the increase of the bottom contribution. The heavy-quark limit can also be obtained by means of a low-energy theorem. The starting point is the Adler-Bell-Jackiw (ABJ) anomaly in the divergence of the axial vector current 93,

$$
\partial^{\mu} j_{\mu}^{5}=2 M_{Q} \bar{Q} i \gamma_{5} Q+\frac{\alpha_{s}}{4 \pi} G^{a \mu \nu} \widetilde{G}_{\mu \nu}^{a}
$$

with $\widetilde{G}_{\mu \nu}^{a}=\frac{1}{2} \epsilon_{\mu \nu \alpha \beta} G^{a \alpha \beta}$ denoting the dual field strength tensor. Since, according to the SutherlandVeltman paradox [94], the matrix element $\left\langle g g\left|\partial^{\mu} j_{\mu}^{5}\right| 0\right\rangle$ vanishes for zero momentum transfer, the matrix element $\left\langle g g\left|M_{Q} \bar{Q} i \gamma_{5} Q\right| 0\right\rangle$ of the Higgs source can be related to the ABJ anomaly in Eq. (68). Thanks to the Adler-Bardeen theorem [95], the ABJ anomaly is not modified by radiative corrections at vanishing momentum transfer, so that the effective Lagrangian

$$
\mathcal{L}_{e f f}=-g_{Q}^{A} \frac{\alpha_{s}}{8 \pi} G^{a \mu \nu} \widetilde{G}_{\mu \nu}^{a} \frac{A}{v}
$$

is valid to all orders of perturbation theory 6 . In order to calculate the full QCD corrections to the $g g$ decay width, this effective coupling has to be inserted in the effective diagrams analogous to those of Fig. 5. The final result agrees with the explicit expansion of the two-loop diagrams in terms of the heavy quark mass at NLO.

The electroweak corrections to the pseudoscalar Higgs decays into gluons have been calculated in the limit of heavy top quarks and $W$ bosons some time ago within the 2HDM, i.e. involving the non-SUSY part of the MSSM. The corrections modify the partial decay width by [96]

$$
\Gamma(A \rightarrow g g)=\Gamma_{L O} \times\left\{1-x_{t}\left(7+\frac{10}{\operatorname{tg}^{2} \beta}\right)\right\}
$$

with $x_{t}$ defined after Eq. (36). The correction ranges at the per-cent level.

For large values of $\operatorname{tg} \beta$ the bottom-quark loops will take over the dominant role. In the limit of small quark masses the form factors develop logarithmic structures,

$$
\begin{aligned}
A_{Q}^{h / H}(\tau) & \rightarrow-\frac{3}{2} \frac{m_{Q}^{2}}{M_{h / H}^{2}}\left[\log \frac{M_{h / H}^{2}}{m_{Q}^{2}}-i \pi\right]^{2} \\
A_{Q}^{A}(\tau) & \rightarrow-\frac{m_{Q}^{2}}{M_{A}^{2}}\left[\log \frac{M_{A}^{2}}{m_{Q}^{2}}-i \pi\right]^{2}
\end{aligned}
$$

so that the quark contributions to the partial decay widths of the scalar and pseudoscalar Higgs bosons approach each other, i.e. the chiral symmetry is restored [the relative factor $3 / 2$ is compensated by the different global coefficients of the partial decay widths of Eqs. (61, 65)]. The same is also true for the NLO QCD corrections [68]. The finite parts of the virtual corrections approach common logarithmic expressions,

$$
\begin{aligned}
\Gamma[\Phi \rightarrow g g] & =\Gamma_{L O}\left\{1+\left(E_{\text {virt }}^{\Phi}+E_{\text {real }}^{\Phi}\right) \frac{\alpha_{s}\left(M_{\Phi}\right)}{\pi}\right\} \\
E_{\text {virt }}^{\Phi}\left(\tau_{Q}\right) & \rightarrow \frac{C_{A}-C_{F}}{12}\left[\log \frac{M_{H}^{2}}{m_{Q}^{2}}-i \pi\right]^{2}-C_{F}\left[\log \frac{M_{H}^{2}}{m_{Q}^{2}}-i \pi\right] \quad \text { for } M_{\Phi}^{2} \gg 4 M_{Q}^{2}
\end{aligned}
$$

with $C_{A}=3$ if the quark mass is defined to be the pole mass. The Abelian part of the large logarithms is related to the Sudakov form factor [97] at the virtual $\Phi b \bar{b}$ vertex and have been resummed down to

\footnotetext{
${ }^{6}$ This has been confirmed explicitly up to NNLO in Ref. 92, if the QCD corrections are expanded in terms of the strong coupling $\alpha_{s}$ with only five light active flavours.
} 
the subleading logarithmic level [98]. The non-Abelian part of these logarithms, however, has not been resummed so far. The real corrections determined by the coefficient $E_{\text {real }}^{\Phi}$ are of subleading non-Abelian logarithmic order.

The genuine SUSY-QCD corrections have been calculated in the limit of heavy SUSY-particle masses by means of a heavy mass expansion for the top-stop-gluino contributions [99, 100, 101, 102] and a mixed heavy mass and large momentum expansion for the bottom-sbottom-gluino corrections [101, 102, 103. A large part of the latter corrections in the bottom case can be absorbed by the effective bottom Yukawa couplings of Eq. (12). However, there is a sizeable remainder. This feature is confirmed by the full calculation of the SUSY-QCD corrections by means of a numerical integration of the corresponding two-loop diagrams [104, 105]. The agreement with the approximate calculations is reasonable. The numerical results of the full calculation are presented in Fig. 7 for the modified small

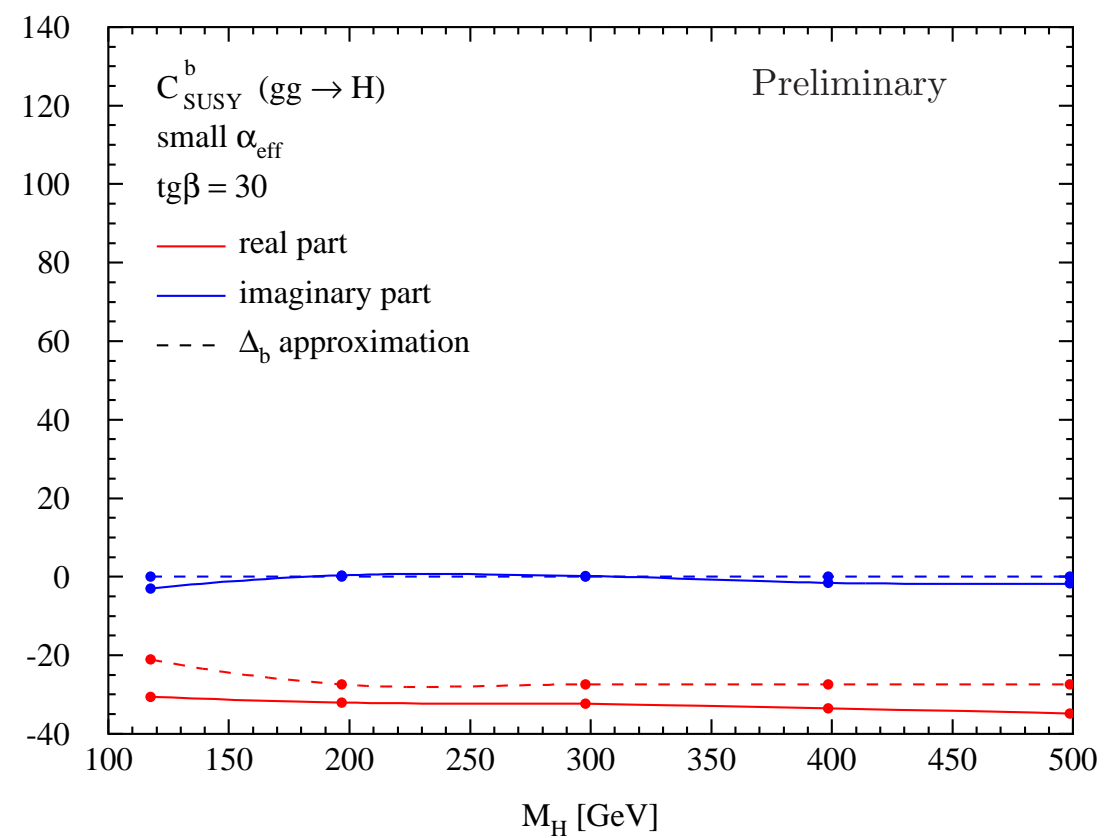

Figure 7: The genuine SUSY-QCD corrections normalized to the LO bottom quark form factor. Real part: red, imaginary part: blue, compared to the $\Delta_{b}$ approximation (dashed lines). $A_{b}$ has been renormalized in the $\overline{M S}$ scheme. From Ref. [105].

$\alpha_{\text {eff }}$ scenario [106], defined by the following choices of MSSM parameters $\left[m_{t}=172.6 \mathrm{GeV}\right]$,

$$
\begin{aligned}
& M_{\tilde{Q}}=800 \mathrm{GeV} \quad \tan \beta=30 \\
& M_{\tilde{g}}=1000 \mathrm{GeV} \quad \mu \quad=2 \mathrm{TeV} \\
& M_{2}=500 \mathrm{GeV} \quad A_{b}=A_{t}=-1.133 \mathrm{TeV} .
\end{aligned}
$$

In this scenario the squark masses amount to

$$
\begin{array}{ll}
m_{\tilde{t}_{1}}=679 \mathrm{GeV} & m_{\tilde{t}_{2}}=935 \mathrm{GeV} \\
m_{\tilde{b}_{1}}=601 \mathrm{GeV} & m_{\tilde{b}_{2}}=961 \mathrm{GeV}
\end{array}
$$

Fig. 7 displays the genuine SUSY-QCD corrections normalized to the LO bottom quark form factor, i.e. $A_{b}^{h / H}\left(\tau_{b}\right) \rightarrow A_{b}^{h / H}\left(\tau_{b}\right)\left(1+C_{S U S Y}^{b} \frac{\alpha_{s}}{\pi}\right)$, where the LO form factor has been defined in terms of the bottom Yukawa coupling $g_{b}^{h / H}$ without $\Delta_{b}$-resummation. The corrections can be sizeable, but can be described reasonably well with the usual $\Delta_{b}$ approximation, if $A_{b}$ is renormalized in the $\overline{\mathrm{MS}}$ scheme, however, with a sizeable remainder that determines the differences between the full and dashed curves of Fig. 7 . 


\subsection{Higgs boson decays into photons}

\subsubsection{Standard Model}
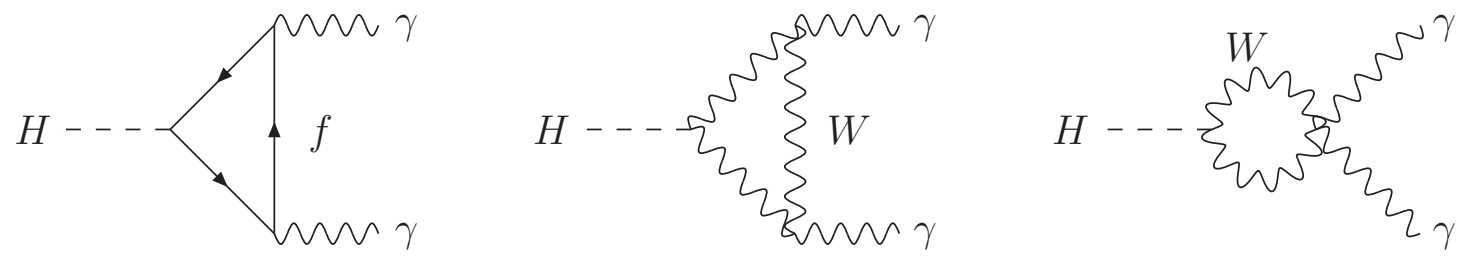

Figure 8: Typical diagrams contributing to $H \rightarrow \gamma \gamma$ at lowest order.

The decay of the Higgs boson into photons is mediated by $W$ and heavy fermion loops in the SM, see Fig. [8, at lowest order the partial decay width is given by [73]

$$
\Gamma[H \rightarrow \gamma \gamma]=\frac{G_{F} \alpha^{2} M_{H}^{3}}{128 \sqrt{2} \pi^{3}}\left|\sum_{f} N_{c f} e_{f}^{2} A_{f}^{H}\left(\tau_{f}\right)+A_{W}^{H}\left(\tau_{W}\right)\right|^{2}
$$

with the form factors

$$
\begin{aligned}
A_{f}^{H}(\tau) & =2 \tau[1+(1-\tau) f(\tau)] \\
A_{W}^{H}(\tau) & =-[2+3 \tau+3 \tau(2-\tau) f(\tau)]
\end{aligned}
$$

and the function $f(\tau)$ defined in Eq. (50). The parameters $\tau_{i}=4 M_{i}^{2} / M_{H}^{2} \quad(i=f, W)$ are defined in terms of the corresponding of the heavy loop-particle masses. For large loop masses the form factors approach constant values:

$$
\begin{array}{ll}
A_{f}^{H} \rightarrow \frac{4}{3} & \text { for } M_{H}^{2} \ll 4 M_{Q}^{2} \\
A_{W}^{H} \rightarrow-7 & \text { for } M_{H}^{2} \ll 4 M_{W}^{2}
\end{array}
$$

The $W$ loop is dominant in the intermediate Higgs mass range, and the fermion loops interfere destructively. Only far above the thresholds, for Higgs masses $M_{H} \sim 600 \mathrm{GeV}$, does the top quark loop become competitive, nearly cancelling the $W$ loop contribution. This feature is still relevant for off-shell Higgs contributions.

In the past the full two-loop QCD corrections to the quark loops have been calculated [68, 107, 108]. The QCD corrections simply rescale the lowest order quark amplitude by a factor that only depends on the ratios of the Higgs and quark masses

$$
\begin{aligned}
& A_{Q}^{H}\left(\tau_{Q}\right) \rightarrow A_{Q}^{H}\left(\tau_{Q}\right) \times\left[1+C_{H}\left(\tau_{Q}\right) \frac{\alpha_{s}}{\pi}\right] \\
& C_{H}\left(\tau_{Q}\right) \rightarrow-1 \quad \text { for } M_{H}^{2} \ll 4 M_{Q}^{2}
\end{aligned}
$$

According to the low-energy theorem discussed before, the QCD corrections in the heavy quark limit can be obtained from the effective Lagrangian [40, 58, 68, 73]

$$
\mathcal{L}_{\text {eff }}=e_{t}^{2} \frac{\alpha}{2 \pi}\left\{(1+\delta) \log \left(1+\frac{H}{v}\right)-\frac{\eta}{2} \log ^{2}\left(1+\frac{H}{v}\right)+\frac{\rho}{3} \log ^{3}\left(1+\frac{H}{v}\right)\right\} F^{\mu \nu} F_{\mu \nu}
$$


with the QCD corrections up to $\mathrm{N}^{3} \mathrm{LO}$

$$
\begin{aligned}
\delta & =\delta_{1} \frac{\alpha_{s}}{\pi}+\delta_{2}\left(\frac{\alpha_{s}}{\pi}\right)^{2}+\delta_{3}\left(\frac{\alpha_{s}}{\pi}\right)^{3}+\mathcal{O}\left(\alpha_{s}^{4}\right) \\
\eta & =\eta_{2}\left(\frac{\alpha_{s}}{\pi}\right)^{2}+\eta_{3}\left(\frac{\alpha_{s}}{\pi}\right)^{3}+\mathcal{O}\left(\alpha_{s}^{4}\right) \\
\rho & =\rho_{3}\left(\frac{\alpha_{s}}{\pi}\right)^{3}+\mathcal{O}\left(\alpha_{s}^{4}\right)
\end{aligned}
$$

The perturbative coefficients are explicitly given by

$$
\begin{aligned}
\delta_{1} & =-1 \\
\delta_{3} & =\frac{7835}{288} \zeta_{3}-\frac{95339}{2592}-\frac{961}{144} L_{t}^{2}-\frac{541}{108} L_{t}+N_{F}\left(\frac{4693}{1296}-\frac{125}{144} \zeta_{3}+\frac{31}{36} L_{t}^{2}+\frac{101}{216} L_{t}\right) \\
& +N_{F}^{2}\left(-\frac{L_{t}^{2}}{36}+\frac{L_{t}}{54}-\frac{19}{324}\right)+\frac{\sum_{i=1}^{N_{F}} e_{q_{i}}^{2}}{e_{t}^{2}}\left[\frac{53}{108}-\frac{55}{54} \zeta_{3}+\frac{11}{54} L_{t}+\frac{29}{72} L_{t}^{2}-N_{F}\left(\frac{449}{3888}+\frac{L_{t}}{36}\right)\right] \\
& +\frac{\left(\sum_{i=1}^{N_{F}+1} e_{q_{i}}^{2}\right)^{2}-\left(\sum_{i=1}^{N_{F}} e_{q_{i}}\right)^{2}}{e_{t}^{2}}\left(\frac{55}{216}-\frac{5}{9} \zeta_{3}\right) \\
\eta_{2}= & \frac{31}{24}-\frac{N_{F}}{6}-\frac{\sum_{i=1}^{N_{F}} e_{q_{i}}^{2}}{e_{t}^{2}} \frac{L_{t}}{6} \\
\eta_{3}= & \frac{541}{108}-\frac{101}{216} N_{F}-\frac{N_{F}^{2}}{54}+\left(\frac{961}{72}-\frac{31}{18} N_{F}+\frac{N_{F}^{2}}{18}\right) L_{t}+\frac{\sum_{i=1}^{N_{F}} e_{q_{i}}^{2}}{e_{t}^{2}}\left(-\frac{11}{54}-\frac{29}{36} L_{t}+\frac{N_{F}}{18} L_{t}\right) \\
\rho_{3}= & -\frac{961}{108}+\frac{31}{27} N_{F}-\frac{N_{F}^{2}}{27}+\frac{\sum_{i=1}^{N_{F}} e_{q_{i}}^{2}}{e_{t}^{2}}\left(\frac{29}{54}-\frac{N_{F}}{27}\right)
\end{aligned}
$$

(where $F_{\mu \nu}$ denotes the photon field strength tensor and $\alpha_{s}$ the strong coupling constant with $N_{F}=5$ active flavours) that can be derived from the top-quark contribution to the kinetic photon operator analogous to the QCD case. The results for $\delta_{2}$ and $\delta_{3}$ agree with previous determinations [75, 109]. Partial results are also known up to $\mathrm{N}^{4} \mathrm{LO}[110]$.

In order to improve the perturbative behaviour of the quark loop contributions they should be expressed preferably in terms of the running quark masses $m_{Q}\left(M_{H} / 2\right)$, which are normalized to the pole masses $M_{Q}$ via

$$
m_{Q}\left(\mu_{Q}=M_{Q}\right)=M_{Q}
$$

with their scale identified with $\mu_{Q}=M_{H} / 2$ within the photonic decay mode. These definitions imply a proper definition of the $Q \bar{Q}$ thresholds $M_{H}=2 M_{Q}$, without artificial displacements due to finite shifts between the pole and running quark masses, as is the case for the running $\overline{\mathrm{MS}}$ masses. The residual QCD corrections are small in the intermediate Higgs-mass range increasing the photonic Higgs decay width by about $2 \%$. Recently the full three-loop QCD corrections to the photonic Higgs decay have been calculated in the heavy top-quark limit [111. They lead to a further contribution of a few per mille.

The two-loop electroweak corrections have been evaluated in Ref. [112] for the $W$-boson and topquark induced contributions. They decrease the partial photonic decay width by about $2 \%$ thus nearly cancelling the NLO QCD corrections. Both types of corrections only become large for Higgs masses above the $t \bar{t}$-threshold, i.e. for $M_{H}>2 m_{t}$. 

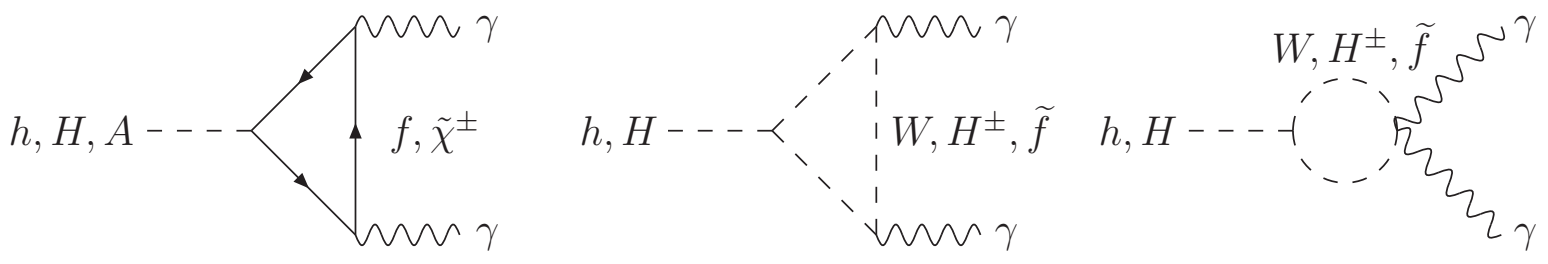

Figure 9: Typical diagrams contributing to $\Phi \rightarrow \gamma \gamma$ at lowest order.

\subsubsection{Minimal supersymmetric extension}

The decays of the scalar Higgs bosons into photons are mediated by $W$ and heavy fermion loops as in the SM and, in addition, by charged Higgs, sfermion and chargino loops, see Fig. 9. The partial decay widths [68, 82] can be expressed as

$$
\begin{aligned}
\Gamma[h / H \rightarrow \gamma \gamma] & =\frac{G_{F} \alpha^{2} M_{h / H}^{3}}{128 \sqrt{2} \pi^{3}} \mid \sum_{f} N_{c f} e_{f}^{2} g_{f}^{h / H} A_{f}^{h / H}\left(\tau_{f}\right)+g_{W}^{h / H} A_{W}^{h / H}\left(\tau_{W}\right) \\
& +g_{H^{ \pm}}^{h / H} A_{H^{ \pm}}^{h / H}\left(\tau_{H^{ \pm}}\right)+\sum_{\tilde{\chi}^{ \pm}} g_{\tilde{\chi}^{ \pm}}^{h / H} A_{\tilde{\chi}^{ \pm}}^{h / H}\left(\tau_{\tilde{\chi}^{ \pm}}\right)+\left.\sum_{\tilde{f}} N_{c f} e_{\tilde{f}}^{2} g_{\tilde{f}}^{h / H} A_{\tilde{f}}^{h / H}\left(\tau_{\tilde{f}}\right)\right|^{2}
\end{aligned}
$$

involving the form factors

$$
\begin{aligned}
A_{f, \tilde{\chi}^{ \pm}}^{h / H}(\tau) & =2 \tau[1+(1-\tau) f(\tau)] \\
A_{H^{ \pm}, \tilde{f}}^{h / H}(\tau) & =-\tau[1-\tau f(\tau)] \\
A_{W}^{h / H}(\tau) & =-[2+3 \tau+3 \tau(2-\tau) f(\tau)]
\end{aligned}
$$

and the function $f(\tau)$ as defined in Eq. (50). For large loop-particle masses the form factors approach constant values,

$$
\begin{array}{ll}
A_{f, \tilde{\chi}^{ \pm}}^{h / H}(\tau) \rightarrow \frac{4}{3} & \text { for } M_{h / H}^{2} \ll 4 M_{f, \tilde{\chi}^{ \pm}}^{2} \\
A_{H^{ \pm}, \tilde{f}}^{h / H}(\tau) \rightarrow \frac{1}{3} & \text { for } M_{h / H}^{2} \ll 4 M_{H^{ \pm}, \tilde{f}}^{2} \\
A_{W}^{h / H}(\tau) \rightarrow-7 & \text { for } M_{h / H}^{2} \ll 4 M_{W}^{2}
\end{array}
$$

Sfermion loops start to be sizeable for sfermion masses $M_{\widetilde{f}} \lesssim 300 \mathrm{GeV}$, while for larger sfermion masses they are negligible in general.

The photonic decay mode of the pseudoscalar Higgs boson is generated by heavy charged-fermion and chargino loops, see Fig. 9. The partial decay width reads [68, 82]

$$
\Gamma(A \rightarrow \gamma \gamma)=\frac{G_{F} \alpha^{2} M_{A}^{3}}{32 \sqrt{2} \pi^{3}}\left|\sum_{f} N_{c f} e_{f}^{2} g_{f}^{A} A_{f}^{A}\left(\tau_{f}\right)+\sum_{\tilde{\chi}^{ \pm}} g_{\tilde{\chi}^{ \pm}}^{A} A_{\tilde{\chi}^{ \pm}}^{A}\left(\tau_{\tilde{\chi}^{ \pm}}\right)\right|^{2}
$$

with the amplitudes

$$
A_{f, \tilde{\chi}^{ \pm}}^{A}(\tau)=\tau f(\tau)
$$


For large loop-particle masses the pseudoscalar amplitudes approach unity. The parameters $\tau_{i}=$ $4 M_{i}^{2} / M_{\Phi}^{2}\left(i=f, W, H^{ \pm}, \tilde{\chi}^{ \pm}, \tilde{f}\right)$ are defined in terms of the corresponding heavy loop-particle masses and the MSSM couplings $g_{f, W, H^{ \pm}, \tilde{\chi}^{ \pm}, \tilde{f}}^{\phi}$ are summarized in Tables 1 and 2 ,

The QCD corrections to the quark and squark loop contributions have been calculated. They are known for finite quark, squark and Higgs masses [68, 83, 107, 108. The QCD corrections rescale the lowest order quark amplitudes [68, 83, 107, 108, 113,

$$
\begin{aligned}
A_{Q}^{\Phi}\left(\tau_{Q}\right) & \rightarrow A_{Q}^{\Phi}\left(\tau_{Q}\right)\left[1+C_{\Phi}\left(\tau_{Q}\right) \frac{\alpha_{s}}{\pi}\right] \\
C_{h / H}\left(\tau_{Q}\right) & \rightarrow-1 \quad \text { for } M_{h / H}^{2} \ll 4 M_{Q}^{2} \\
C_{A}\left(\tau_{Q}\right) & \rightarrow 0 \quad \text { for } M_{A}^{2} \ll 4 M_{Q}^{2} \\
A_{\widetilde{Q}}^{h / H}\left(\tau_{\widetilde{Q}}\right) & \rightarrow A_{\widetilde{Q}}^{h / H}\left(\tau_{\widetilde{Q}}\right)\left[1+\widetilde{C}_{h / H}\left(\tau_{\widetilde{Q}}\right) \frac{\alpha_{s}}{\pi}\right] \\
\widetilde{C}_{h / H}\left(\tau_{\widetilde{Q}}\right) & \rightarrow 3 \quad \text { for } M_{h / H}^{2} \ll 4 M_{\widetilde{Q}}^{2}
\end{aligned}
$$

The QCD corrections to the $\gamma \gamma$ decay width are defined in terms of the running quark masses in the same way as the SM photonic decay width. The QCD radiative corrections are moderate in the intermediate mass range [68, 107, 108, where this decay mode will be important. Owing to the narrow-width approximation of the virtual quarks, the QCD corrections to the pseudoscalar (scalar) decay width exhibit a Coulomb singularity at the $t \bar{t}(\tilde{Q} \tilde{\tilde{Q}})$ threshold, which is regularized by taking into account the finite top quark and squarks decay widths [91].

The QCD corrections to the quark loops in the heavy quark limit can be obtained by means of the low-energy theorems for scalar as well as pseudoscalar Higgs particles, which have been discussed before. The result for the scalar Higgs bosons agrees with the SM result of Eq. (777), and the QCD corrections to the pseudoscalar decay mode vanish in this limit due to the Adler-Bardeen theorem [95]. In complete analogy to the gluonic decay mode, the effective Lagrangian can be derived from the ABJ anomaly [93] and is given to all orders of perturbation theory by [68]

$$
\mathcal{L}_{\text {eff }}=-g_{Q}^{A} e_{Q}^{2} \frac{3 \alpha}{4 \pi} F^{\mu \nu} \widetilde{F}_{\mu \nu} \frac{A}{v}
$$

Since there are no effective diagrams generated by light particle interactions that contribute to the photonic decay width at next-to-leading order, the QCD corrections to the pseudoscalar decay width vanish, in agreement with the explicit expansion of the massive two-loop result. Starting at NNLO there are light quark-loop induced contributions involving the effective Agg-vertex in the heavy-quark limit.

Completely analogous the QCD corrections to the squark loops for the scalar Higgs particles in the heavy squark limit can be obtained by the extension of the scalar low-energy theorem to the scalar squarks. Their coupling to photons at NLO can be described by the effective Lagrangian [113]

$$
\mathcal{L}_{e f f}=g_{\widetilde{Q}}^{H} e_{\widetilde{Q}}^{2} \frac{\alpha}{8 \pi} F^{\mu \nu} F_{\mu \nu} \frac{H}{v}\left[1+3 \frac{\alpha_{s}}{\pi}\right]
$$

where only non-SUSY-QCD corrections are taken into account, i.e. diagrams with gluino exchange or the squark four-point couplings have been omitted. The genuine SUSY-QCD corrections can be deduced from the analogous gluon-gluon case as described and mentioned in Refs. [100, 102, 103. Their numerical impact, however, has not been analysed so far.

\footnotetext{
${ }^{7}$ Taking into account the squark four-point couplings for degenerate squark masses, too, the coefficient 3 is changed to $10 / 3$ [81, 83, 84].
} 
The electroweak corrections to the pseudoscalar Higgs decays into photons have been calculated in the limit of heavy top quarks and $W$ bosons some time ago within the 2HDM, i.e. involving the non-SUSY part of the MSSM. The corrections modify the partial decay width by [96]

$$
\Gamma(A \rightarrow \gamma \gamma)=\Gamma_{L O} \times\left\{1-x_{t}\left(4+\frac{7}{\operatorname{tg}^{2} \beta}\right)\right\}
$$

with $x_{t}$ defined after Eq. (36). The correction ranges at the per-cent level.

For large values of $\operatorname{tg} \beta$ the bottom-quark loops will take over the dominant role. In the limit of small quark masses the form factors develop logarithmic structures,

$$
\begin{aligned}
A_{Q}^{h / H}(\tau) & \rightarrow-2 \frac{m_{Q}^{2}}{M_{h / H}^{2}}\left[\log \frac{M_{h / H}^{2}}{m_{Q}^{2}}-i \pi\right]^{2} \\
A_{Q}^{A}(\tau) & \rightarrow-\frac{m_{Q}^{2}}{M_{A}^{2}}\left[\log \frac{M_{A}^{2}}{m_{Q}^{2}}-i \pi\right]^{2}
\end{aligned}
$$

so that the quark contributions to the partial decay widths of the scalar and pseudoscalar Higgs bosons approach each other, i.e. the chiral symmetry is restored [the relative factor 2 is compensated by the different global coefficients of the partial decay widths of Eqs. [82, 83)]. The same is also true for the NLO QCD corrections,

$$
C_{\phi}\left(\tau_{Q}\right) \rightarrow C_{F}\left\{-\frac{1}{24}\left[\log \frac{M_{\phi}^{2}}{m_{Q}^{2}}-i \pi\right]^{2}-\frac{1}{2}\left[\log \frac{M_{\phi}^{2}}{m_{Q}^{2}}-i \pi\right]+\frac{3}{2} \log \frac{\mu_{R}^{2}}{m_{Q}^{2}}\right\} \quad \text { for } M_{\phi}^{2} \gg 4 M_{Q}^{2}
$$

where $\mu_{R}$ denotes the renormalization scale of the running quark mass. The large logarithms are related to the Sudakov form factor [97] at the virtual $\Phi b \bar{b}$ vertex and have been resummed down to the subleading logarithmic level [98].

\subsection{Higgs boson decays into photon and $Z$ boson and Dalitz decays}

\subsubsection{Standard Model}
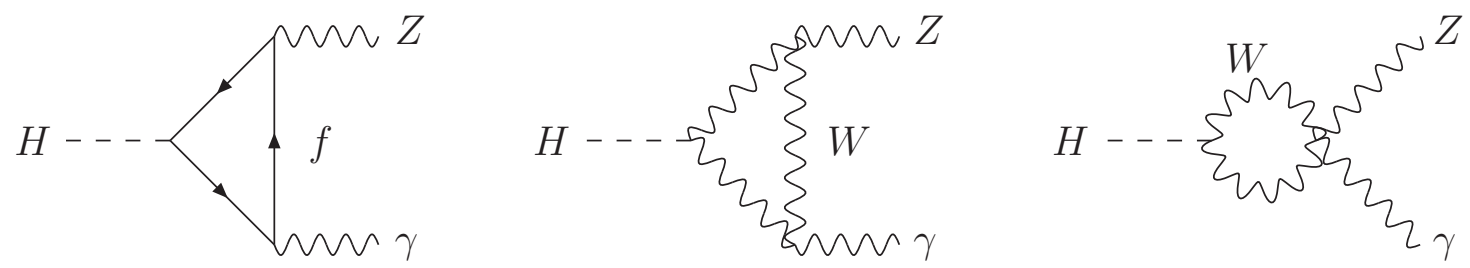

Figure 10: Typical diagrams contributing to $H \rightarrow Z \gamma$ at lowest order.

On-shell $Z$ boson. The decay of the Higgs boson into a photon and a $Z$ boson proceeds via $W$ and heavy fermion loops, see Fig. 10, The partial decay width can be obtained at leading order as [82, 114]

$$
\Gamma[H \rightarrow Z \gamma]=\frac{G_{F}^{2} M_{W}^{2} \alpha M_{H}^{3}}{64 \pi^{4}}\left(1-\frac{M_{Z}^{2}}{M_{H}^{2}}\right)^{3}\left|\sum_{f} A_{f}^{H}\left(\tau_{f}, \lambda_{f}\right)+A_{W}^{H}\left(\tau_{W}, \lambda_{W}\right)\right|^{2}
$$


with the $W$ and fermion form factors

$$
\begin{aligned}
A_{f}^{H}(\tau, \lambda)= & 2 N_{c f} \frac{e_{f}\left(I_{3 f}-2 e_{f} \sin ^{2} \theta_{W}\right)}{\cos \theta_{W}}\left[I_{1}(\tau, \lambda)-I_{2}(\tau, \lambda)\right] \\
A_{W}^{H}(\tau, \lambda)= & \cos \theta_{W}\left\{4\left(3-\tan ^{2} \theta_{W}\right) I_{2}(\tau, \lambda)\right. \\
& \left.+\left[\left(1+\frac{2}{\tau}\right) \tan ^{2} \theta_{W}-\left(5+\frac{2}{\tau}\right)\right] I_{1}(\tau, \lambda)\right\}
\end{aligned}
$$

The functions $I_{1}, I_{2}$ are defined as

$$
\begin{aligned}
I_{1}(\tau, \lambda) & =\frac{\tau \lambda}{2(\tau-\lambda)}+\frac{\tau^{2} \lambda^{2}}{2(\tau-\lambda)^{2}}[f(\tau)-f(\lambda)]+\frac{\tau^{2} \lambda}{(\tau-\lambda)^{2}}[g(\tau)-g(\lambda)] \\
I_{2}(\tau, \lambda) & =-\frac{\tau \lambda}{2(\tau-\lambda)}[f(\tau)-f(\lambda)]
\end{aligned}
$$

where the function $g(\tau)$ is given by

$$
g(\tau)= \begin{cases}\sqrt{\tau-1} \arcsin \frac{1}{\sqrt{\tau}} & \tau \geq 1 \\ \frac{\sqrt{1-\tau}}{2}\left[\log \frac{1+\sqrt{1-\tau}}{1-\sqrt{1-\tau}}-i \pi\right] & \tau<1\end{cases}
$$

and $f(\tau)$ is defined in Eq. (50). The parameters $\tau_{i}=4 M_{i}^{2} / M_{H}^{2}$ and $\lambda_{i}=4 M_{i}^{2} / M_{Z}^{2} \quad(i=f, W)$ are defined in terms of the corresponding masses of the heavy loop particles. Due to charge conjugation invariance, only the vectorial $Z$ coupling contributes to the fermion loop so that problems with the axial $\gamma_{5}$ coupling do not arise. The $W$ loop provides the dominant contribution in the intermediate Higgs mass range, and the heavy fermion loops interfere destructively.

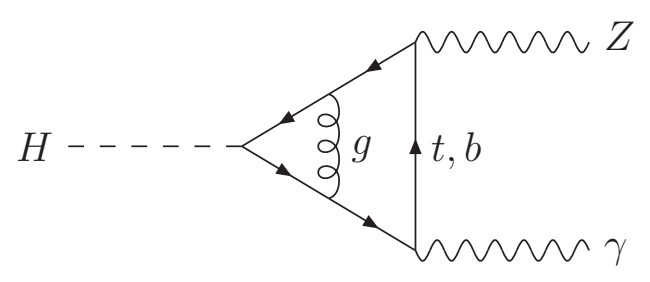

Figure 11: Typical diagram contributing to the $Q C D$ corrections to $H \rightarrow Z \gamma$.

The two-loop QCD corrections to the top quark loops have been calculated numerically [115] and analytically very recently [116]. They are generated by virtual gluon exchange inside the quark triangle (see Fig. 11). Due to charge conjugation invariance and color conservation, radiation of a single gluon is not possible. Hence the QCD corrections can simply be expressed as a rescaling of the lowest order amplitude by a factor that only depends on the ratios $\tau_{i}$ and $\lambda_{i} \quad(i=f, W)$, defined above:

$$
\begin{aligned}
A_{Q}^{H}\left(\tau_{Q}, \lambda_{Q}\right) & \rightarrow A_{Q}^{H}\left(\tau_{Q}, \lambda_{Q}\right) \times\left[1+D_{H}\left(\tau_{Q}, \lambda_{Q}\right) \frac{\alpha_{s}}{\pi}\right] \\
D_{H}\left(\tau_{Q}, \lambda_{Q}\right) & \rightarrow-1 \quad \text { for } M_{Z}^{2} \ll M_{H}^{2} \ll 4 M_{Q}^{2}
\end{aligned}
$$

In the limit $M_{Z} \rightarrow 0$ the quark amplitude approaches the corresponding form factor of the photonic decay mode (modulo couplings), which has been discussed before. Hence the QCD correction in the 
heavy quark limit for small $Z$ masses has to coincide with the heavy quark limit of the photonic decay mode of Eq. (177). The QCD corrections for finite Higgs, $Z$ and quark masses are presented in [115, 116] as a function of the Higgs mass. They amount to less than $0.3 \%$ in the intermediate mass range, where this decay mode is relevant, and can thus be neglected. More important is the inclusion of off-shell effects of the final-state $Z$ boson due to its finite width. This requires the analysis of Dalitz decays $H \rightarrow \gamma f \bar{f}$.
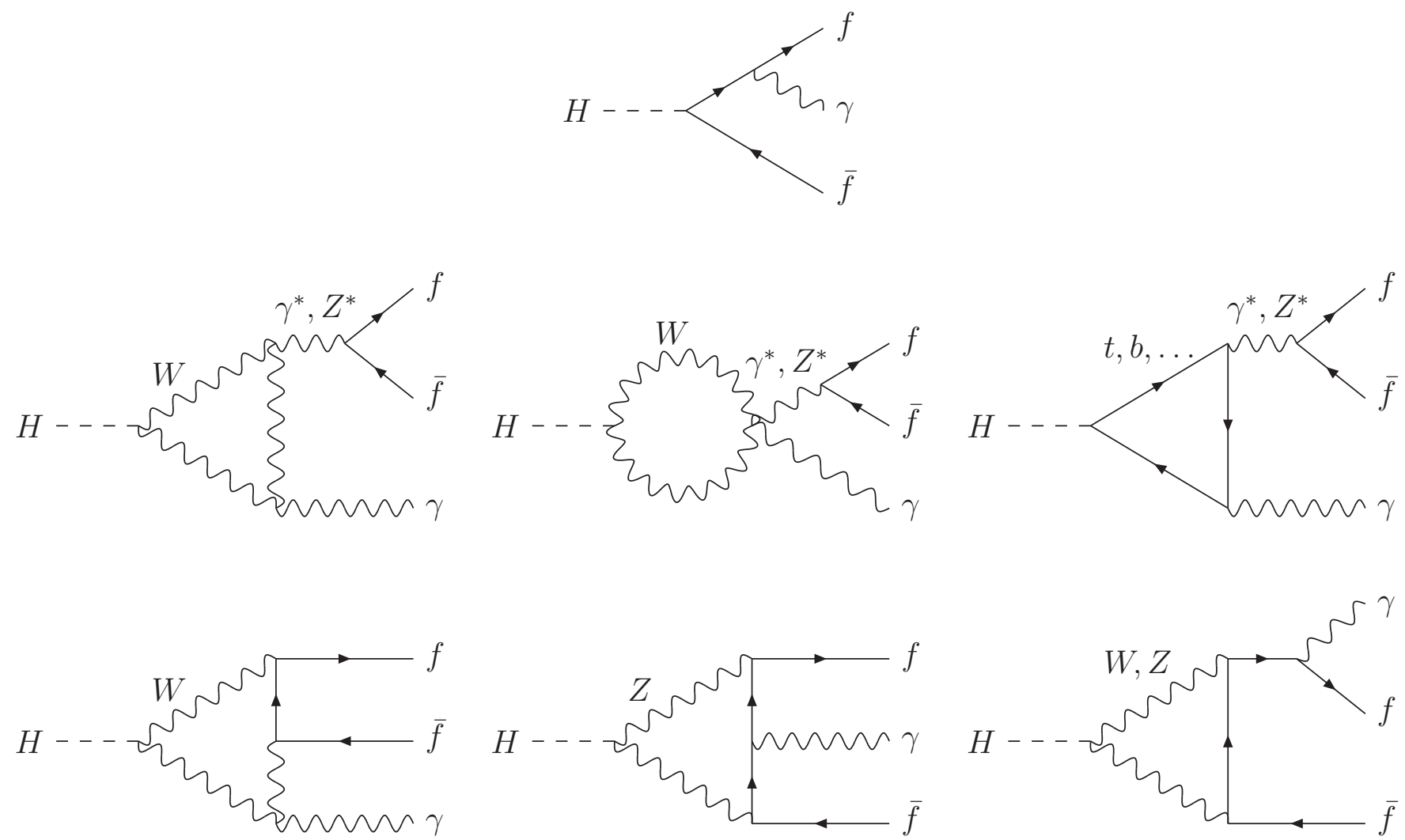

Figure 12: Generic diagrams contributing to the Dalitz decays $H \rightarrow \gamma f \bar{f}$.

Dalitz decays. Dalitz decays of the Higgs boson [117, 118] are described by the diagrams of Fig. 12. The first diagram corresponds to the direct contributions, since these are mediated by the Yukawa coupling of the fermion $f$. The second line shows the triangle diagrams which include the resonant $H \rightarrow \gamma Z \rightarrow \gamma f \bar{f}$ contribution as well as photon conversion $H \rightarrow \gamma \gamma^{*} \rightarrow \gamma f \bar{f}$. The leading contributions to the triangles emerge from $W$, top and bottom loops. The last line exhibits box contributions and finalstate photon radiation which, however, are numerically suppressed compared to the triangle diagrams, but are required for a gauge-invariant result.

Fig. 13] shows the invariant mass distributions for $e^{+} e^{-}, \mu^{+} \mu^{-}$and $\tau^{+} \tau^{-}$pairs including the individual contributions of the different types of diagrams normalized to the partial decay width into photons. A lower cut of $E_{\gamma}>1 \mathrm{GeV}$ has been imposed on the photon energy to avoid the infrared singularity of photon radiation. For small invariant masses the photon conversion $H \rightarrow \gamma \gamma^{*} \rightarrow \gamma \ell^{+} \ell^{-}$provides the dominant contribution, while for invariant masses around the $Z$-boson mass the $Z$-boson contribution $H \rightarrow \gamma Z^{*} \rightarrow \gamma f \bar{f}$ takes over the dominant role. At the end-point $q^{2} \lesssim M_{H}^{2}$ of the spectrum the direct contribution determines the distributions. This rises with growing Yukawa coupling, i.e. it is largest for $H \rightarrow \gamma \tau^{+} \tau^{-}$(where it dominates in the whole $q^{2}$-range). For a clean separation of the $H \rightarrow \gamma \gamma$, $H \rightarrow \gamma \gamma^{*} \rightarrow \gamma \ell^{+} \ell^{-}, H \rightarrow Z \gamma$ and $H \rightarrow \ell^{+} \ell^{-}$contributions appropriate cuts have to be implemented for the Dalitz decays. The low- $q^{2}$ part has to be attributed to $H \rightarrow \gamma \gamma$, the $q^{2}$-part around $M_{Z}^{2}$ to $H \rightarrow Z \gamma$ and the end-point region close to $M_{H}^{2}$ to the QED corrections to $H \rightarrow \ell^{+} \ell^{-}$. 


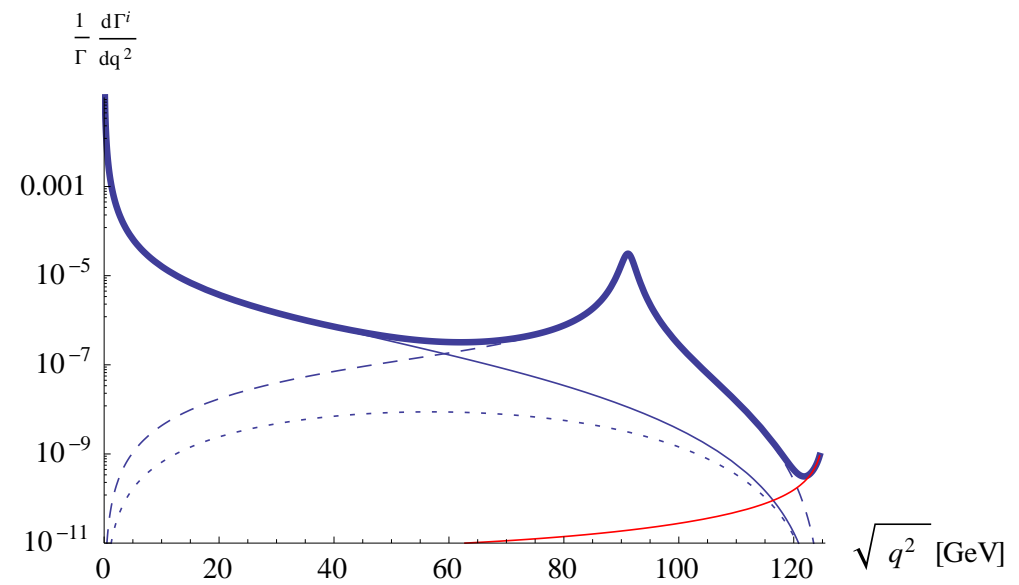

$$
H \rightarrow \gamma e^{+} e^{-}
$$
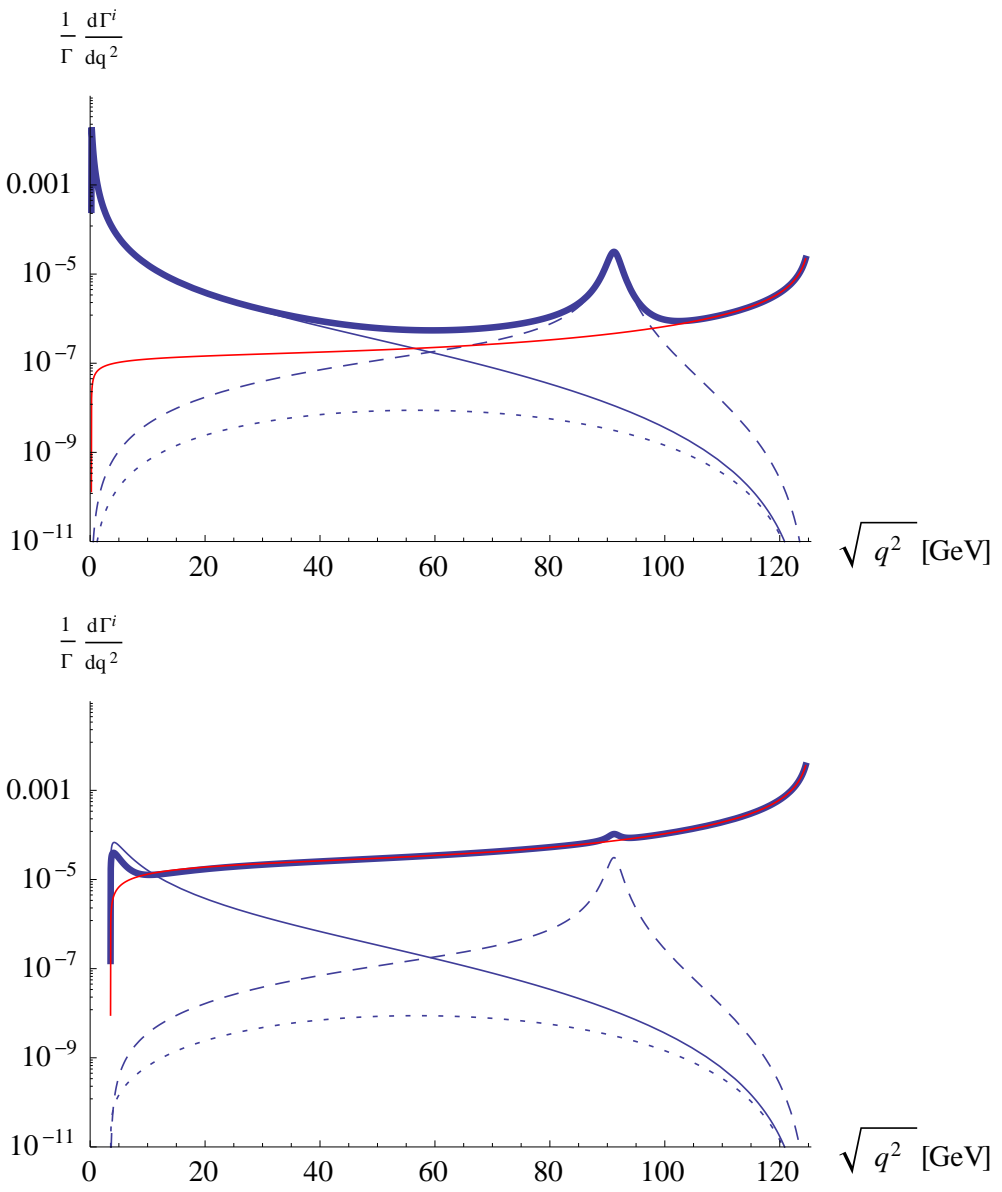

$H \rightarrow \gamma \mu^{+} \mu^{-}$

$$
H \rightarrow \gamma \tau^{+} \tau^{-}
$$

Figure 13: The invariant mass distributions in $\sqrt{q^{2}}=M_{\ell^{+} \ell^{-}}$of the Dalitz decays $H \rightarrow \gamma+$ $e^{+} e^{-} / \mu^{+} \mu^{-} / \tau^{+} \tau^{-}$normalized to $\Gamma(H \rightarrow \gamma \gamma)$ with a cut $E_{\gamma}>1$ GeV on the photon energy. The red lines show the contribution of the tree diagrams, the thin solid lines denote the contribution of the photon conversion $H \rightarrow \gamma \gamma^{*} \rightarrow \gamma \ell^{+} \ell^{-}$, and the dashed line the contribution from the $Z^{*}$ exchange diagrams, while the thick lines present the total contributions. The dotted lines denote the contribution from the box diagrams (in 't Hooft-Feynman gauge). From Ref. [118]. 


\subsubsection{Minimal supersymmetric extension}
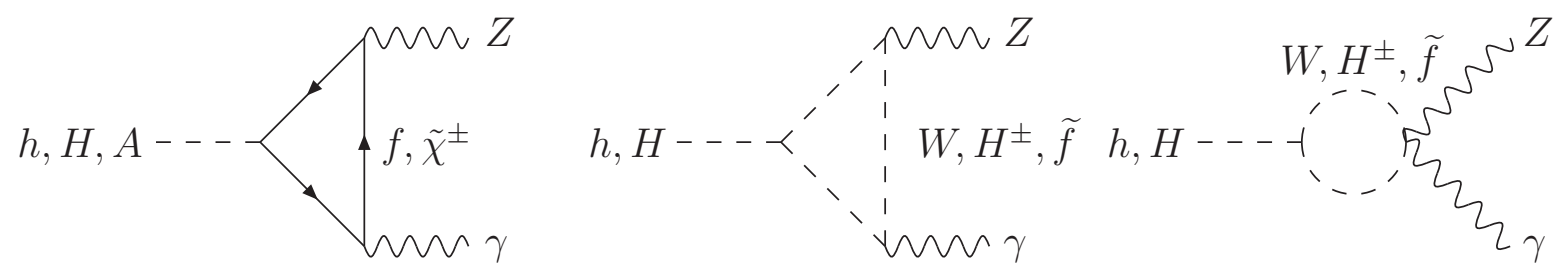

Figure 14: Typical diagrams contributing to $\Phi \rightarrow Z \gamma$ at lowest order.

On-shell $Z$ boson. The decays of the scalar Higgs bosons into a $Z$ boson and photon are mediated by $W$ and heavy fermion loops as in the Standard Model and, in addition, by charged Higgs, sfermion and chargino loops as shown in Fig. 14. The partial decay widths are given by [68, 119]

$$
\begin{aligned}
\Gamma[h / H \rightarrow Z \gamma]= & \frac{G_{F}^{2} M_{W}^{2} \alpha M_{h / H}^{3}}{64 \pi^{4}}\left(1-\frac{M_{Z}^{2}}{M_{h / H}^{2}}\right)^{3} \mid \sum_{f} g_{f}^{h / H} A_{f}^{h / H}\left(\tau_{f}, \lambda_{f}\right) \\
& +g_{W}^{h / H} A_{W}^{h / H}\left(\tau_{W}, \lambda_{W}\right)+g_{H^{ \pm}}^{h / H} A_{H^{ \pm}}^{h / H}\left(\tau_{H^{ \pm}}, \lambda_{H^{ \pm}}\right) \\
& +\sum_{\tilde{\chi}_{i}^{ \pm}, \tilde{\chi}_{j}^{\mp}} g_{\tilde{\chi}_{i}^{ \pm} \tilde{\chi}_{j}^{\mp}}^{h / H} g_{\tilde{\chi}_{i}^{\mp} \tilde{\chi}_{j}^{ \pm}}^{Z} A_{\tilde{\chi}_{i}^{ \pm} \tilde{\chi}_{j}^{\mp}}^{h / H}+\left.\sum_{\tilde{f}_{i}, \tilde{f}_{j}} g_{\tilde{f}_{i} \tilde{f}_{j}}^{h / H} g_{\tilde{f}_{i} \tilde{f}_{j}}^{Z} A_{\tilde{f}_{i} \tilde{f}_{j}}^{h / H}\right|^{2}
\end{aligned}
$$

with the form factors $A_{f}^{h / H}, A_{W}^{h / H}$ of Eq. (93), and

$$
A_{H^{ \pm}}^{h / H}(\tau, \lambda)=\frac{\cos 2 \theta_{W}}{\cos \theta_{W}} I_{1}(\tau, \lambda)
$$

where the function $I_{1}(\tau, \lambda)$ is defined after Eq. (93).

The $Z \gamma$ decay mode of the pseudoscalar Higgs boson is generated by heavy charged-fermion and chargino loops, see Fig. 14. The partial decay width reads [119]

$$
\Gamma(A \rightarrow Z \gamma)=\frac{G_{F}^{2} M_{W}^{2} \alpha M_{A}^{3}}{64 \pi^{4}}\left(1-\frac{M_{Z}^{2}}{M_{A}^{2}}\right)^{3}\left|\sum_{f} g_{f}^{A} A_{f}^{A}\left(\tau_{f}, \lambda_{f}\right)+\sum_{\tilde{\chi}_{i}^{ \pm}, \tilde{\chi}_{j}^{\mp}} g_{\tilde{\chi}_{i}^{ \pm}}^{A} \tilde{\chi}_{j}^{\mp} g_{\tilde{\chi}_{i}^{\mp} \tilde{\chi}_{j}^{ \pm}}^{Z} A_{\tilde{\chi}_{i}^{ \pm} \tilde{\chi}_{j}^{ \pm}}^{A}\right|^{2}
$$

with the fermion amplitudes

$$
A_{f}^{A}(\tau, \lambda)=2 N_{c f} \frac{e_{f}\left(I_{3 f}-2 e_{f} \sin ^{2} \theta_{W}\right)}{\cos \theta_{W}} I_{2}(\tau, \lambda)
$$

The contributions of charginos and sfermions involve mixing terms. Their analytical expressions can be found in [119]. For large loop-particle masses and small $Z$-boson mass, the form factors approach the photonic amplitudes modulo couplings. The parameters $\tau_{i}=4 M_{i}^{2} / M_{\Phi}^{2}, \lambda_{i}=4 M_{i}^{2} / M_{Z}^{2} \quad(i=$ $\left.f, W, H^{ \pm}, \tilde{\chi}^{ \pm}, \tilde{f}\right)$ are defined by the corresponding heavy loop-particle masses and the non-mixing MSSM couplings $g_{f, W, H^{ \pm}, \tilde{\chi}^{ \pm}, \tilde{f}}$ are summarized in Tables 1 and 2, while the mixing and $Z$ boson couplings $g_{i}^{Z}$ can be found in [82]. The branching ratios of the $Z \gamma$ decay modes range at a level of up to a few $10^{-4}$ in the intermediate mass ranges of the Higgs bosons and are thus phenomenologically less important in the MSSM. Dalitz decays have not been studied within the MSSM framework. They involve additional contributions from charged Higgs bosons, charginos and sfermions in the loop contributions including the box diagrams. 


\subsection{Supersymmetric Higgs boson decays into Higgs particles}
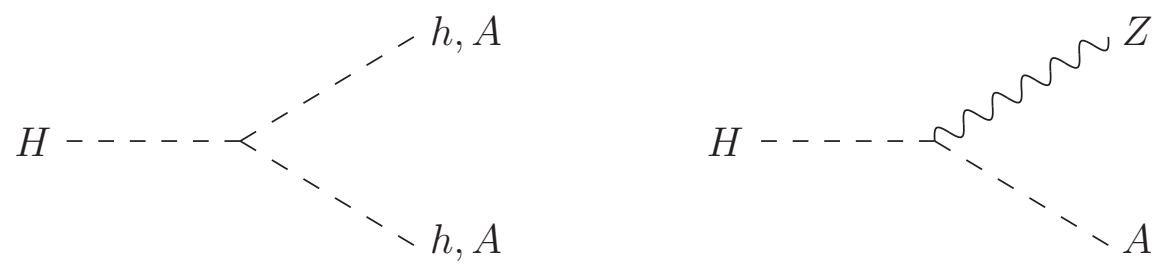

Figure 15: Typical diagrams contributing to Higgs decays with Higgs bosons in the final state.

The heavy scalar Higgs boson can decay into pairs of light scalar as well as pseudoscalar Higgs bosons, see Fig. 15. At LO the partial decay widths are given by [82]

$$
\begin{aligned}
\Gamma(H \rightarrow h h) & =\lambda_{H h h}^{2} \frac{G_{F} M_{Z}^{4}}{16 \sqrt{2} \pi M_{H}} \sqrt{1-4 \frac{M_{h}^{2}}{M_{H}^{2}}} \\
\Gamma(H \rightarrow A A) & =\lambda_{H A A}^{2} \frac{G_{F} M_{Z}^{4}}{16 \sqrt{2} \pi M_{H}} \sqrt{1-4 \frac{M_{A}^{2}}{M_{H}^{2}}}
\end{aligned}
$$

The self-couplings $\lambda_{H h h}$ and $\lambda_{H A A}$ can be derived from the effective Higgs potential [33, 34]. The decay mode into pseudoscalar particles is restricted to small regions of the MSSM parameter space, where the pseudoscalar mass $M_{A}$ is small. The decay into light scalar bosons is dominant for $\operatorname{small} \operatorname{tg} \beta$ below the $t \bar{t}$ threshold. The SUSY-electroweak corrections to these decays have been calculated [120]. They modify the partial decay widths by a moderate amount in general depending on the MSSM scenario if the radiatively improved trilinear Higgs couplings and masses are used, absorbing in this way the bulk of the corrections in terms of effective parameters. Only in parameter regions where the LO decay widths are suppressed the radiative corrections can be larger, but there these decay modes are phenomenologically irrelevant.

The contributions of final states containing off-shell scalar or pseudoscalar Higgs bosons may be significant and are thus included in HdecAy [54] and FeynHiggs [121]. Their expressions read as [56]

$$
\begin{aligned}
\Gamma\left(H \rightarrow \phi \phi^{*}\right)= & \lambda_{H \phi \phi}^{2} g_{\phi b b}^{2} m_{b}^{2} \frac{3 G_{F}^{2} M_{Z}^{4}}{32 \pi^{3} M_{H}}\left\{\left(\kappa_{\phi}-1\right)\left(2-\frac{1}{2} \log \kappa_{\phi}\right)\right. \\
& \left.+\frac{1-5 \kappa_{\phi}}{\sqrt{4 \kappa_{\phi}-1}}\left(\arctan \frac{2 \kappa_{\phi}-1}{\sqrt{4 \kappa_{\phi}-1}}-\arctan \frac{1}{\sqrt{4 \kappa_{\phi}-1}}\right)\right\}
\end{aligned}
$$

where $\kappa_{\phi}=M_{\phi}^{2} / M_{H}^{2}$. They slightly extend the regions, where the $h h, A A$ decay modes of the heavy scalar Higgs boson $H$ are sizeable. Analogous to the decays into $W$ and $Z$ boson pairs double off-shell decays play a role in some regions of the MSSM parameter space below the corresponding thresholds. Neglecting the final-state fermion masses they can be cast into the form [122]

$$
\Gamma\left(H \rightarrow \phi^{*} \phi^{*}\right)=\frac{1}{\pi^{2}} \int_{0}^{M_{H}^{2}} \frac{d Q_{1}^{2} M_{\phi} \Gamma_{\phi}}{\left(Q_{1}^{2}-M_{\phi}^{2}\right)^{2}+M_{\phi}^{2} \Gamma_{\phi}^{2}} \int_{0}^{\left(M_{H}-Q_{1}\right)^{2}} \frac{d Q_{2}^{2} M_{\phi} \Gamma_{\phi}}{\left(Q_{2}^{2}-M_{\phi}^{2}\right)^{2}+M_{\phi}^{2} \Gamma_{\phi}^{2}} \Gamma_{0}
$$

with $Q_{1}^{2}, Q_{2}^{2}$ being the squared invariant masses of the virtual Higgs bosons, $M_{\phi}$ and $\Gamma_{\phi}$ their masses and total decay widths; $\Gamma_{0}$ is given by 122

$$
\Gamma_{0}=\lambda_{H \phi \phi}^{2} \frac{G_{F} M_{Z}^{4}}{16 \sqrt{2} \pi M_{H}} \sqrt{\lambda\left(Q_{1}^{2}, Q_{2}^{2} ; M_{H}^{2}\right)} \frac{Q_{1}^{2} Q_{2}^{2}}{M_{\phi}^{4}}
$$


with the phase-space factor $\lambda(x, y ; z)$ of Eq. (39).

Moreover, Higgs bosons can decay into a gauge and a Higgs boson, see Fig. 15, The various partial widths can be expressed as

$$
\begin{aligned}
\Gamma(H \rightarrow A Z) & =\lambda_{H A Z}^{2} \frac{G_{F} M_{H}^{3}}{8 \sqrt{2} \pi} \lambda^{3 / 2}\left(M_{A}^{2}, M_{Z}^{2} ; M_{H}^{2}\right) \\
\Gamma\left(H \rightarrow H^{ \pm} W^{\mp}\right) & =\lambda_{H H^{+} W}^{2} \frac{G_{F} M_{H}^{3}}{8 \sqrt{2} \pi} \lambda^{3 / 2}\left(M_{H^{ \pm}}^{2}, M_{W}^{2} ; M_{H}^{2}\right) \\
\Gamma(A \rightarrow h Z) & =\lambda_{h A Z}^{2} \frac{G_{F} M_{A}^{3}}{8 \sqrt{2} \pi} \lambda^{3 / 2}\left(M_{h}^{2}, M_{Z}^{2} ; M_{A}^{2}\right) \\
\Gamma\left(H^{+} \rightarrow h W^{+}\right) & =\lambda_{h H^{+} W}^{2} \frac{G_{F} M_{H^{ \pm}}^{3}}{8 \sqrt{2} \pi} \lambda^{3 / 2}\left(M_{h}^{2}, M_{W}^{2} ; M_{H^{ \pm}}^{2}\right)
\end{aligned}
$$

where the couplings $\lambda_{i j k}$ can be determined from the effective Higgs potential [33, 34]. The branching ratios of these decay modes may be sizeable in specific regions of the MSSM parameter space. The electroweak corrections within the 2HDM framework have been calculated [123. They are of moderate size if the radiatively corrected Higgs couplings and masses are used at tree-level. The genuine SUSYelectroweak corrections are only known for the effective parameters $\lambda_{i j k}$ and the Higgs masses.

Below-threshold decays into a Higgs particle and an off-shell gauge boson turn out to be very important for the heavy Higgs bosons of the MSSM. The individual contributions read as [56, 122]

$$
\begin{aligned}
\Gamma\left(H \rightarrow A Z^{*}\right) & =\lambda_{H A Z}^{2} \delta_{Z}^{\prime} \frac{9 G_{F}^{2} M_{Z}^{4} M_{H}}{8 \pi^{3}} G_{A Z} \\
\Gamma\left(H \rightarrow H^{ \pm} W^{\mp *}\right) & =\lambda_{H H^{ \pm} W}^{2} \frac{9 G_{F}^{2} M_{W}^{4} M_{H}}{16 \pi^{3}} G_{H^{ \pm} W} \\
\Gamma\left(A \rightarrow h Z^{*}\right) & =\lambda_{h A Z}^{2} \delta_{Z}^{\prime} \frac{9 G_{F}^{2} M_{Z}^{4} M_{A}}{8 \pi^{3}} G_{h Z} \\
\Gamma\left(H^{+} \rightarrow h W^{+*}\right) & =\lambda_{h H^{ \pm} W}^{2} \frac{9 G_{F}^{2} M_{W}^{4} M_{H^{ \pm}}}{16 \pi^{3}} G_{h W} \\
\Gamma\left(H^{+} \rightarrow A W^{+*}\right) & =\frac{9 G_{F}^{2} M_{W}^{4} M_{H^{ \pm}}}{16 \pi^{3}} G_{A W}
\end{aligned}
$$

with the generic functions $G_{i j}$

$$
\begin{aligned}
G_{i j}= & \frac{1}{4}\left\{2\left(-1+\kappa_{j}-\kappa_{i}\right) \sqrt{\lambda_{i j}}\left[\frac{\pi}{2}+\arctan \left(\frac{\kappa_{j}\left(1-\kappa_{j}+\kappa_{i}\right)-\lambda_{i j}}{\left(1-\kappa_{i}\right) \sqrt{\lambda_{i j}}}\right)\right]\right. \\
& \left.+\left(\lambda_{i j}-2 \kappa_{i}\right) \log \kappa_{i}+\frac{1}{3}\left(1-\kappa_{i}\right)\left[5\left(1+\kappa_{i}\right)-4 \kappa_{j}+\frac{2}{\kappa_{j}} \lambda_{i j}\right]\right\}
\end{aligned}
$$

using the parameters

$$
\lambda_{i j}=-1+2 \kappa_{i}+2 \kappa_{j}-\left(\kappa_{i}-\kappa_{j}\right)^{2}, \quad \quad \kappa_{i}=\frac{M_{i}^{2}}{M_{\phi}^{2}}
$$

with $\delta_{Z}^{\prime}=7 / 12-10 \sin ^{2} \theta_{W} / 9+40 \sin ^{4} \theta_{W} / 27$. Off-shell $h Z^{*}$ decays are important for the pseudoscalar Higgs boson for masses above about $130 \mathrm{GeV}$ for small $\operatorname{tg} \beta$ [56]. The decay modes $H^{ \pm} \rightarrow h W^{*}, A W^{*}$ can reach branching ratios of several tens of per cent and lead to a significant reduction of the dominant branching ratio into $\tau \nu$ final states to a level of $60-70 \%$ for small $\operatorname{tg} \beta$ [56]. This analysis can be extended 
to the double off-shell cases analogous to the previous decay modes into Higgs-boson pairs. If final-state fermion masses are neglected the corresponding expressions are given by

$$
\Gamma\left(\Phi \rightarrow \phi^{*} V^{*}\right)=\frac{1}{\pi^{2}} \int_{0}^{M_{\Phi}^{2}} \frac{d Q_{1}^{2} M_{\phi} \Gamma_{\phi}}{\left(Q_{1}^{2}-M_{\phi}^{2}\right)^{2}+M_{\phi}^{2} \Gamma_{\phi}^{2}} \int_{0}^{\left(M_{\Phi}-Q_{1}\right)^{2}} \frac{d Q_{2}^{2} M_{V} \Gamma_{V}}{\left(Q_{2}^{2}-M_{V}^{2}\right)^{2}+M_{V}^{2} \Gamma_{V}^{2}} \Gamma_{0}
$$

with $Q_{1}^{2}, Q_{2}^{2}$ being the squared invariant masses of the virtual Higgs and gauge bosons, $M_{\phi}, M_{V}$ and $\Gamma_{\phi}, \Gamma_{V}$ their masses and total decay widths; $\Gamma_{0}$ is given by

$$
\Gamma_{0}=\lambda_{\Phi \phi V}^{2} \frac{G_{F} M_{\Phi}^{3}}{8 \sqrt{2} \pi} \lambda^{3 / 2}\left(Q_{1}^{2}, Q_{2}^{2} ; M_{\Phi}^{2}\right)
$$

with the phase-space factor $\lambda(x, y ; z)$ of Eq. (39).

\section{7 $\quad$ Supersymmetric Higgs boson decays}

Sfermion masses and couplings. The scalar partners $\tilde{f}_{L, R}$ of the left- and right-handed fermion components mix with each other. The mass eigenstates $\tilde{f}_{1,2}$ of the sfermions $\tilde{f}$ are related to the current eigenstates $\tilde{f}_{L, R}$ by mixing angles $\theta_{f}$,

$$
\begin{aligned}
& \tilde{f}_{1}=\tilde{f}_{L} \cos \theta_{f}+\tilde{f}_{R} \sin \theta_{f} \\
& \tilde{f}_{2}=-\tilde{f}_{L} \sin \theta_{f}+\tilde{f}_{R} \cos \theta_{f}
\end{aligned}
$$

which are proportional to the masses of the related fermions. Thus mixing effects are only important for the third-generation sfermions $\tilde{t}, \tilde{b}, \tilde{\tau}$, the mass matrix of which is given by [86]

$$
\mathcal{M}_{\tilde{f}}=\left[\begin{array}{cc}
\tilde{M}_{\tilde{f}_{L}}^{2}+M_{f}^{2} & M_{f}\left(A_{f}-\mu r_{f}\right) \\
M_{f}\left(A_{f}-\mu r_{f}\right) & \tilde{M}_{\tilde{f}_{R}}^{2}+M_{f}^{2}
\end{array}\right]
$$

with the parameters $r_{b}=r_{\tau}=1 / r_{t}=\operatorname{tg} \beta$. The parameters $A_{f}$ denote the trilinear Yukawa mixing

parameters of the soft supersymmetry breaking part of the Lagrangian and $\tilde{M}_{\tilde{f}_{L / R}}$ contain the soft SUSY-breaking squark-mass parameters $M_{\tilde{f}_{L / R}}$ and $D$-terms,

$$
\begin{aligned}
\tilde{M}_{\tilde{f}_{L / R}}^{2} & =M_{\tilde{f}_{L / R}}^{2}+D_{\tilde{f}_{L / R}} \\
D_{\tilde{f}_{L}} & =M_{Z}^{2}\left(I_{3 L}^{f}-e_{f} \sin ^{2} \theta_{W}\right) \cos 2 \beta \\
D_{\tilde{f}_{R}} & =M_{Z}^{2} e_{f} \sin ^{2} \theta_{W} \cos 2 \beta
\end{aligned}
$$

The mixing angles acquire the explicit form

$$
\sin 2 \theta_{f}=\frac{2 M_{f}\left(A_{f}-\mu r_{f}\right)}{M_{\tilde{f}_{1}}^{2}-M_{\tilde{f}_{2}}^{2}} \quad, \quad \cos 2 \theta_{f}=\frac{\tilde{M}_{\tilde{f}_{L}}^{2}-\tilde{M}_{\tilde{f}_{R}}^{2}}{M_{\tilde{f}_{1}}^{2}-M_{\tilde{f}_{2}}^{2}}
$$

and the masses of the squark eigenstates are given by

$$
M_{\tilde{f}_{1,2}}^{2}=M_{f}^{2}+\frac{1}{2}\left[\tilde{M}_{\tilde{f}_{L}}^{2}+\tilde{M}_{\tilde{f}_{R}}^{2} \mp \sqrt{\left(\tilde{M}_{\tilde{f}_{L}}^{2}-\tilde{M}_{\tilde{f}_{R}}^{2}\right)^{2}+4 M_{f}^{2}\left(A_{f}-\mu r_{f}\right)^{2}}\right]
$$

The neutral Higgs couplings to sfermions can be derived as [124]

$$
\begin{aligned}
g_{\tilde{f}_{L} \tilde{f}_{L}}^{\Phi} & =M_{f}^{2} g_{1}^{\Phi}+M_{Z}^{2}\left(I_{3 f}-e_{f} \sin ^{2} \theta_{W}\right) g_{2}^{\Phi} \\
g_{\tilde{f}_{R} \tilde{f}_{R}}^{\Phi} & =M_{f}^{2} g_{1}^{\Phi}+M_{Z}^{2} e_{f} \sin ^{2} \theta_{W} g_{2}^{\Phi} \\
g_{\tilde{f}_{L} \tilde{f}_{R}}^{\Phi} & =-\frac{M_{f}}{2}\left(\mu g_{3}^{\Phi}-A_{f} g_{4}^{\Phi}\right)
\end{aligned}
$$




\begin{tabular}{|c|c||c|c|c|c|}
\hline$\tilde{f}$ & $\Phi$ & $g_{1}^{\Phi}$ & $g_{2}^{\Phi}$ & $g_{3}^{\Phi}$ & $g_{4}^{\Phi}$ \\
\hline \hline \multirow{4}{*}{} & $h$ & $\cos \alpha / \sin \beta$ & $-\sin (\alpha+\beta)$ & $-\sin \alpha / \sin \beta$ & $\cos \alpha / \sin \beta$ \\
& $H$ & $\sin \alpha / \sin \beta$ & $\cos (\alpha+\beta)$ & $\cos \alpha / \sin \beta$ & $\sin \alpha / \sin \beta$ \\
& $A$ & 0 & 0 & 1 & $-1 / \operatorname{tg} \beta$ \\
\hline & $h$ & $-\sin \alpha / \cos \beta$ & $-\sin (\alpha+\beta)$ & $\cos \alpha / \cos \beta$ & $-\sin \alpha / \cos \beta$ \\
$\tilde{d}$ & $H$ & $\cos \alpha / \cos \beta$ & $\cos (\alpha+\beta)$ & $\sin \alpha / \cos \beta$ & $\cos \alpha / \cos \beta$ \\
& $A$ & 0 & 0 & 1 & $-\operatorname{tg} \beta$ \\
\hline
\end{tabular}

Table 3: Coefficients of the neutral MSSM Higgs couplings to sfermion pairs.

with the couplings $g_{i}^{\Phi}$ listed in Table 3. The charged Higgs couplings to sfermion pairs [124] can be expressed as $(\alpha, \beta=L, R)$

$$
g_{\tilde{u}_{\alpha} \tilde{d}_{\beta}}^{H^{ \pm}}=-\frac{1}{\sqrt{2}}\left[g_{1}^{\alpha \beta}+M_{W}^{2} g_{2}^{\alpha \beta}\right]
$$

with the coefficients $g_{1,2}^{\alpha \beta}$ summarized in Table 4.

\begin{tabular}{|c||c|c|c|c|}
\hline$i$ & $g_{i}^{L L}$ & $g_{i}^{R R}$ & $g_{i}^{L R}$ & $g_{i}^{R L}$ \\
\hline \hline 1 & $M_{u}^{2} / \operatorname{tg} \beta+M_{d}^{2} \operatorname{tg} \beta$ & $M_{u} M_{d}(\operatorname{tg} \beta+1 / \operatorname{tg} \beta)$ & $M_{d}\left(\mu+A_{d} \operatorname{tg} \beta\right)$ & $M_{u}\left(\mu+A_{u} / \operatorname{tg} \beta\right)$ \\
2 & $-\sin 2 \beta$ & 0 & 0 & 0 \\
\hline
\end{tabular}

Table 4: Coefficients of the charged MSSM Higgs couplings to sfermion pairs.

In the past SUSY-QCD corrections have been computed to the squark masses [125, 126]. They are significant in several MSSM parameter regions. The basic outline of these radiative corrections can be discussed starting from the $\overline{\mathrm{MS}}$ parameters in the squark mass matrix at LO,

$$
\mathcal{M}_{\tilde{q}}=\left[\begin{array}{cc}
\tilde{\bar{M}}_{\tilde{q}_{L}}^{2}\left(Q_{0}\right)+\hat{m}_{q}^{2}\left(Q_{0}\right) & \hat{m}_{q}\left(Q_{0}\right)\left[\bar{A}_{q}\left(Q_{0}\right)-\mu r_{q}\right] \\
\hat{m}_{q}\left(Q_{0}\right)\left[\bar{A}_{q}\left(Q_{0}\right)-\mu r_{q}\right] & \tilde{\tilde{M}}_{\tilde{q}_{R}}^{2}\left(Q_{0}\right)+\hat{m}_{q}^{2}\left(Q_{0}\right)
\end{array}\right]
$$

where $\bar{A}_{q}\left(Q_{0}\right)$ denotes the running trilinear $\overline{\mathrm{MS}}$ coupling and $\tilde{\bar{M}}_{\tilde{q}_{L / R}}^{2}\left(Q_{0}\right)$ the left- and right-handed soft SUSY-breaking squark $\overline{\mathrm{MS}}$ mass parameters $\bar{M}_{\tilde{q}_{L / R}}\left(Q_{0}\right)$ at the scale $Q_{0}$ plus the corresponding $D$-terms [see Eq. (121)]. In order to accommodate the large $\Delta_{b}$ terms in the sbottom mass matrix the effective bottom-mass parameter is defined as

$$
\hat{m}_{b}(Q)=\frac{\bar{m}_{b}(Q)}{1+\Delta_{b}}
$$

where $\bar{m}_{b}(Q)$ represents the usual $\overline{\mathrm{MS}}$ bottom mass at the scale $Q$. For the stop mass matrix $\hat{m}_{t}(Q)$ is just identified with the $\overline{\mathrm{MS}}$ top mass, $\hat{m}_{t}(Q)=\bar{m}_{t}(Q)$.

At NLO the masses of the stop/sbottom eigenstates are given by

$$
m_{\tilde{q}_{1 / 2}}^{2}=\hat{m}_{q}^{2}\left(Q_{0}\right)+\frac{1}{2}\left[\tilde{\bar{M}}_{\tilde{q}_{L}}^{2}\left(Q_{0}\right)+\tilde{\bar{M}}_{\tilde{q}_{R}}^{2}\left(Q_{0}\right)\right.
$$




$$
\begin{aligned}
& \left.\mp \sqrt{\left[\tilde{\bar{M}}_{\tilde{q}_{L}}^{2}\left(Q_{0}\right)-\tilde{\bar{M}}_{\tilde{q}_{R}}^{2}\left(Q_{0}\right)\right]^{2}+4 \hat{m}_{q}^{2}\left(Q_{0}\right)\left[\bar{A}_{q}\left(Q_{0}\right)-\mu r_{q}\right]^{2}}\right]+\Delta m_{\tilde{q}_{1 / 2}}^{2} \\
& \Delta m_{\tilde{q}_{1 / 2}}^{2}=\Sigma_{11 / 22}\left(m_{\tilde{q}_{1 / 2}}^{2}\right)+\delta \hat{m}_{\tilde{q}_{1 / 2}}^{2}
\end{aligned}
$$

where $\Sigma_{11 / 22}$ denote the diagonal parts of the stop/sbottom self-energies and $\delta \hat{m}_{\tilde{q}_{1 / 2}}^{2}$ the mass counter terms. At LO the mixing angles are defined by

$$
\sin 2 \tilde{\theta}_{q}=\frac{2 \hat{m}_{q}\left(Q_{0}\right)\left[\bar{A}_{q}\left(Q_{0}\right)-\mu r_{q}\right]}{m_{\tilde{q}_{1}}^{2}-m_{\tilde{q}_{2}}^{2}} \quad, \quad \cos 2 \tilde{\theta}_{q}=\frac{\tilde{M}_{\tilde{q}_{L}}^{2}\left(Q_{0}\right)-\tilde{M}_{\tilde{q}_{R}}^{2}\left(Q_{0}\right)}{m_{\tilde{q}_{1}}^{2}-m_{\tilde{q}_{2}}^{2}}
$$

The radiative corrections to the diagonal matrix elements are compensated by shifts in the soft mass parameters $\bar{M}_{\tilde{q}_{L / R}}\left(Q_{0}\right)$,

$$
\tilde{M}_{\tilde{q}_{L / R}}^{2}\left(Q_{0}\right)=\tilde{\bar{M}}_{\tilde{q}_{L / R}}^{2}\left(Q_{0}\right)+\Delta \bar{M}_{\tilde{q}_{L / R}}^{2}
$$

in order to arrive at tree-level-like expressions at NLO for the stop/sbottom masses. The shifted SUSYmass parameters are determined from the sum rules

$$
\begin{aligned}
& \tilde{M}_{\tilde{q}_{L}}^{2}\left(Q_{0}\right)=m_{\tilde{q}_{1}}^{2} \cos ^{2} \tilde{\theta}_{q}+m_{\tilde{q}_{2}}^{2} \sin ^{2} \tilde{\theta}_{q}-\hat{m}_{q}^{2}\left(Q_{0}\right) \\
& \tilde{M}_{\tilde{q}_{R}}^{2}\left(Q_{0}\right)=m_{\tilde{q}_{1}}^{2} \sin ^{2} \tilde{\theta}_{q}+m_{\tilde{q}_{2}}^{2} \cos ^{2} \tilde{\theta}_{q}-\hat{m}_{q}^{2}\left(Q_{0}\right)
\end{aligned}
$$

The tree-level definition of the mixing angle $\tilde{\theta}_{q}$ in Eq. (129) corresponds to the following counter term at NLO,

$$
\begin{gathered}
\delta \tilde{\theta}_{q}=\frac{\operatorname{tg} 2 \tilde{\theta}_{q}}{2}\left\{\frac{\delta \hat{m}_{q}}{\hat{m}_{q}\left(Q_{0}\right)}+\frac{\delta \bar{A}_{q}}{\bar{A}_{q}\left(Q_{0}\right)-\mu r_{q}}-\frac{\delta m_{\tilde{q}_{1}}^{2}-\delta m_{\tilde{q}_{2}}^{2}}{m_{\tilde{q}_{1}}^{2}-m_{\tilde{q}_{2}}^{2}}\right\} \\
\delta m_{\tilde{q}_{1 / 2}}^{2}=-\Sigma_{11 / 22}\left(m_{\tilde{q}_{1 / 2}}^{2}\right)
\end{gathered}
$$

with the $\overline{\mathrm{MS}}$ counter term $\delta \bar{A}_{q}$, while $\delta \hat{m}_{q}$ includes the finite $\Delta_{b}$ contributions in addition. However, in order to avoid artificial singularities in physical observables for stop/sbottom masses $m_{\tilde{q}_{1,2}}$ close to each other, in HDECAY the mixing angle of the squark fields has been renormalized via the anti-Hermitian counter term [125, 127,

$$
\delta \theta_{q}=\frac{1}{2} \frac{\Re e \Sigma_{12}\left(m_{\tilde{q}_{1}}^{2}\right)+\Re e \Sigma_{12}\left(m_{\tilde{q}_{2}}^{2}\right)}{m_{\tilde{q}_{2}}^{2}-m_{\tilde{q}_{1}}^{2}}
$$

where $\Sigma_{12}$ denotes the off-diagonal part of the stop/sbottom self-energy describing transitions from the first to the second mass eigenstate or vice versa. This implies a finite shift $\Delta \tilde{\theta}_{q}$ to the mixing angle $\tilde{\theta}_{q}$ of Eq. (129),

$$
\theta_{q}=\tilde{\theta}_{q}+\Delta \tilde{\theta}_{q} \quad, \quad \Delta \tilde{\theta}_{q}=\delta \tilde{\theta}_{q}-\delta \theta_{q}
$$

which modifies the relations of Eq. (131) by replacing $\tilde{\theta}_{q}=\theta_{q}-\Delta \tilde{\theta}_{q}$.

Decays into sleptons and squarks. The sfermionic decay widths of the MSSM Higgs bosons $H_{k}$ ( $k=1,2,3,4$ corresponds to $H, h, A, H^{ \pm}$and $\left.i, j=1,2\right)$ can be written as [124]

$$
\Gamma\left(H_{k} \rightarrow \tilde{f}_{i} \overline{\tilde{f}}_{j}\right)=\frac{N_{c} G_{F}}{2 \sqrt{2} \pi M_{H_{k}}} \sqrt{\lambda_{\tilde{f}_{i} \tilde{f}_{j}, H_{k}}}\left(g_{\tilde{f}_{i} \tilde{f}_{j}}^{H_{k}}\right)^{2}
$$

where $N_{c}=3(1)$ for squarks (sleptons). The physical MSSM couplings $g_{\tilde{f}_{i} \tilde{f}_{j}}^{H_{k}}$ can be obtained from the couplings shown in Eqs. (124) and (125) by means of the mixing relations in Eq. (119). The symbol 
$\lambda_{i j, k}=\lambda\left(M_{i}^{2}, M_{j}^{2} ; M_{k}^{2}\right)$ denotes the usual two-body phase-space factor of Eq. (39). In the limit of massless fermions, which is a valid approximation for the first two generations, the pseudoscalar Higgs boson $A$ does not decay into sfermions due to the suppression of sfermion mixing by the fermion mass. In the decoupling regime, where the Higgs masses $M_{H, H^{ \pm}}$are large, the decay widths of the heavy scalar and charged Higgs particles into sfermions are proportional to [124]

$$
\Gamma\left(H, H^{ \pm} \rightarrow \tilde{\tilde{f}} \overline{\tilde{f}}\right) \propto \frac{G_{F} M_{W}^{4}}{M_{H, H^{ \pm}}} \sin ^{2} 2 \beta
$$

They are only important for small $\operatorname{tg} \beta \sim 1$. However, they are suppressed by an inverse power of the large Higgs masses, rendering unimportant the sfermion decays of the first two generations.

Decay widths into third-generation sfermions $(\tilde{t}, \tilde{b}, \tilde{\tau})$ can be much larger, thanks to the significantly larger fermion masses. For instance, in the asymptotic regime the heavy scalar Higgs decay into stop pairs of the same helicity is proportional to [124]

$$
\Gamma(H \rightarrow \overline{\tilde{t}}) \propto \frac{G_{F} M_{t}^{4}}{M_{H} \operatorname{tg}^{2} \beta}
$$

which will be enhanced by large coefficients compared to the first/second-generation squarks for small $\operatorname{tg} \beta$. At large $\operatorname{tg} \beta$ sbottom decays will be significant. Moreover, for large Higgs masses the decay widths of heavy neutral CP-even and CP-odd Higgs particles into stop pairs of different helicity will be proportional to [124]

$$
\Gamma(H, A \rightarrow \tilde{\tilde{t}}) \propto \frac{G_{F} M_{t}^{2}}{M_{H, A}}\left[\mu+\frac{A_{t}}{\operatorname{tg} \beta}\right]^{2}
$$

and hence will be of the same order of magnitude as standard fermion and chargino/neutralino decay widths. In summary, if third-generation sfermion decays are kinematically allowed, they have to be taken into account.

Some time ago the SUSY-QCD corrections to the stop and sbottom decays of the MSSM Higgs bosons have been calculated [125, 126, 127]. They reach about 20-40\%, but are larger close to the threshold regions due to the Coulomb singularities. It is relevant to resum the large $\Delta_{b}$ terms as e.g. discussed in the previous paragraph in order to absorb the bulk of the higher-order corrections in effective bottom and sbottom parameters. These corrections are included in HDECAY [54, FEYNHigGS [121] and HFOLD [128. The SUSY-electroweak corrections are known, too, and modify the partial decay widths by less than about 10\% [129]. They are included in FeynHiggs and HFold.

Chargino/neutralino masses and couplings. The chargino/neutralino masses and couplings to the MSSM Higgs bosons are derived from the Higgs mass parameter $\mu$ and the $\mathrm{SU}(2)$ gaugino mass parameter $M_{2}$. The mass matrix of the charginos reads as [86]

$$
\mathcal{M}_{\chi^{ \pm}}=\left[\begin{array}{cc}
M_{2} & \sqrt{2} M_{W} \sin \beta \\
\sqrt{2} M_{W} \cos \beta & \mu
\end{array}\right]
$$

It can be diagonalized by two mixing matrices $U, V$, yielding the masses of the physical $\chi_{1,2}^{ \pm}$states:

$$
\begin{aligned}
M_{\chi_{1,2}^{ \pm}}= & \frac{1}{\sqrt{2}}\left\{M_{2}^{2}+\mu^{2}+2 M_{W}^{2}\right. \\
& \left.\mp \sqrt{\left(M_{2}^{2}-\mu^{2}\right)^{2}+4 M_{W}^{4} \cos ^{2} 2 \beta+4 M_{W}^{2}\left(M_{2}^{2}+\mu^{2}+2 M_{2} \mu \sin 2 \beta\right)}\right\}^{1 / 2}
\end{aligned}
$$


If either $\mu$ or $M_{2}$ is large, one chargino corresponds to a pure gaugino state and the other to a pure higgsino state. The Higgs couplings to charginos [124, 130] can be expressed as $(k=1,2,3,4$ corresponds to $\left.H, h, A, H^{ \pm}\right)$

$$
H_{k} \rightarrow \chi_{i}^{+} \chi_{j}^{-}: \quad F_{i j k}=\frac{1}{\sqrt{2}}\left[e_{k} V_{i 1} U_{j 2}-d_{k} V_{i 2} U_{j 1}\right]
$$

where the coefficients $e_{k}$ and $d_{k}$ are determined as

$$
\begin{aligned}
& e_{1}=\cos \alpha \\
& d_{1}=-\sin \alpha, e_{2}=\sin \alpha, d_{2}=\cos \alpha, \quad e_{3}=-\sin \beta \\
& d_{3}=\cos \beta
\end{aligned}
$$

The mass matrix of the four neutralinos depends in addition on the $\mathrm{U}(1)$ gaugino mass parameter $M_{1}$, which is constrained by SUGRA models to be $M_{1}=\frac{5}{3} \tan \theta_{W} M_{2}$. In the bino-wino-higgsino basis, it acquires the form [86]

$$
\mathcal{M}_{\chi^{0}}=\left[\begin{array}{cccc}
M_{1} & 0 & -M_{Z} \sin \theta_{W} \cos \beta & M_{Z} \sin \theta_{W} \sin \beta \\
0 & M_{2} & M_{Z} \cos \theta_{W} \cos \beta & -M_{Z} \cos \theta_{W} \sin \beta \\
-M_{Z} \sin \theta_{W} \cos \beta & M_{Z} \cos \theta_{W} \cos \beta & 0 & -\mu \\
M_{Z} \sin \theta_{W} \sin \beta & -M_{Z} \cos \theta_{W} \sin \beta & -\mu & 0
\end{array}\right]
$$

which can be diagonalized by a single mixing matrix $N$. The final results are too involved to be presented here. They can be found in [124]. If either $\mu$ or $M_{2}$ is large, two neutralinos are pure gaugino states and the other two pure higgsino states. The Higgs couplings to neutralino pairs [124, 130] can be written as $(k=1,2,3$ corresponds to $H, h, A)$

$$
H_{k} \rightarrow \chi_{i}^{0} \chi_{j}^{0}: \quad F_{i j k}=\frac{1}{2}\left(N_{j 2}-\tan \theta_{W} N_{j 1}\right)\left(e_{k} N_{i 3}+d_{k} N_{i 4}\right)+(i \leftrightarrow j)
$$

with the coefficients $e_{k}, d_{k}$ defined in Eq. (143).

The charged Higgs couplings to chargino-neutralino pairs are fixed to be [124]

$$
\begin{aligned}
H^{ \pm} \rightarrow \chi_{i}^{ \pm} \chi_{j}^{0}: \quad F_{i j 4} & =\cos \beta\left[V_{i 1} N_{j 4}+\frac{1}{\sqrt{2}} V_{i 2}\left(N_{j 2}+\tan \theta_{W} N_{j 1}\right)\right] \\
F_{j i 4} & =\sin \beta\left[U_{i 1} N_{j 3}-\frac{1}{\sqrt{2}} U_{i 2}\left(N_{j 2}+\tan \theta_{W} N_{j 1}\right)\right]
\end{aligned}
$$

Analogous to the previous Higgs decays into sfermions the gaugino masses and couplings can be loopcorrected by NLO electroweak effects via shifts of the SUSY parameters involved in the derivation of these masses and couplings [131]. The electroweak corrections to the gaugino sector are of moderate size.

Decays into charginos and neutralinos. The decay widths of the MSSM Higgs particles $H_{k}(k=$ $1,2,3,4$ corresponds to $\left.H, h, A, H^{ \pm}\right)$into neutralino and chargino pairs can be cast into the form [124, 130

$$
\begin{aligned}
\Gamma\left(H_{k} \rightarrow \chi_{i} \chi_{j}\right)= & \frac{G_{F} M_{W}^{2}}{2 \sqrt{2} \pi} \frac{M_{H_{k}} \sqrt{\lambda_{i j, k}}}{1+\delta_{i j}}\left[\left(F_{i j k}^{2}+F_{j i k}^{2}\right)\left(1-\frac{M_{\chi_{i}}^{2}}{M_{H_{k}}^{2}}-\frac{M_{\chi_{j}}^{2}}{M_{H_{k}}^{2}}\right)\right. \\
& \left.-4 \eta_{k} \epsilon_{i} \epsilon_{j} F_{i j k} F_{j i k} \frac{M_{\chi_{i}} M_{\chi_{j}}}{M_{H_{k}}^{2}}\right]
\end{aligned}
$$

where $\eta_{1,2,4}=+1, \eta_{3}=-1$ and $\delta_{i j}=0$ unless the final state consists of two identical (Majorana) neutralinos, in which case $\delta_{i i}=1 ; \epsilon_{i}= \pm 1$ stands for the sign of the $i$ 'th eigenvalue of the neutralino 
mass matrix, which can be positive or negative. For charginos these parameters are always equal to unity. The symbols $\lambda_{i j, k}=\lambda\left(M_{\chi_{i}}^{2}, M_{\chi_{j}}^{2} ; M_{\chi_{k}}^{2}\right)$ denote the usual two-body phase-space functions of Eq. (39).

If chargino/neutralino decays are kinematically allowed, which may be the case for the heavy MSSM Higgs particles $H, A, H^{ \pm}$, their branching ratios can reach large values below the corresponding top quark thresholds. They can thus jeopardize the Higgs search at the LHC due to the invisibility of a significant fraction of these decay modes [124]. Even above the corresponding top quark thresholds the chargino/neutralino branching ratios can be sizeable. For large Higgs masses they can reach common values of a couple of $10 \%$. In the asymptotic regime $M_{H_{k}} \gg M_{\chi}$, the total sum of decay widths into charginos and neutralinos acquires the simple form [124, 130]

$$
\Gamma\left(H_{k} \rightarrow \sum_{i, j} \chi_{i} \chi_{j}\right)=\frac{3 G_{F} M_{W}^{2}}{4 \sqrt{2} \pi} M_{H_{k}}\left(1+\frac{1}{3} \tan ^{2} \theta_{W}\right)
$$

for all three Higgs bosons $H, A, H^{ \pm}$, which is independent of any MSSM parameter $\left(\operatorname{tg} \beta, \mu, A_{t, b}, M_{2}\right)$. Normalized to the total width, which is dominated by $t \bar{t}, b \bar{b}(t \bar{b})$ decay modes for the neutral (charged) Higgs particles the branching ratio of chargino/neutralino decays will exceed a level of about $20 \%$ even for small and large $\operatorname{tg} \beta$. In some part of the MSSM parameter space, invisible light scalar Higgs boson decays into the lightest neutralino $h \rightarrow \chi_{1}^{0} \chi_{1}^{0}$ will be possible with a relevant branching ratio [124, 130].

The full SUSY-electroweak corrections to the Higgs boson decays into gaugino pairs have been calculated [131. Apart from parameter regions where the LO decay width is suppressed they are of moderate size, i.e. not larger than about 20\%. It should be noted that a consistent renormalization of the gaugino sector is mandatory for reliable predictions of these partial decay widths.

\subsection{Branching ratios and total decay width}

\subsubsection{Standard Model}

The determination of the branching ratios of Higgs-boson decays requires the inclusion of the aforementioned higher-order corrections and a sophisticated estimate of the theoretical and parametric uncertainties. The first analyses of this type have been performed some time ago [132] and recently [65, 133]. The parametric uncertainties are dominated by the uncertainties in the top, bottom and charm quark masses as well as the strong coupling $\alpha_{s}$. We have used the $\overline{\mathrm{MS}}$ masses for the bottom and charm quark [51],

$$
\bar{m}_{b}\left(\bar{m}_{b}\right)=(4.18 \pm 0.03) \mathrm{GeV}, \quad \bar{m}_{c}(3 \mathrm{GeV})=(0.986 \pm 0.026) \mathrm{GeV}
$$

and the top quark pole mass

$$
M_{t}=(172.5 \pm 1) \mathrm{GeV}
$$

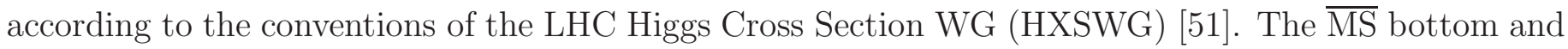
charm masses are evolved from the input scale to the scale of the decay process with 4-loop accuracy in QCD. If needed the pole mass of the bottom quark is obtained from the 3-loop conversion between the $\overline{\mathrm{MS}}$ and pole mass [134],

$$
\begin{aligned}
M_{b}= & \overline{m_{b}}\left(\mu_{R}^{2}\right)\left\{1+\left(\frac{4}{3}+\log \frac{\mu_{R}^{2}}{M_{b}^{2}}\right) \frac{\alpha_{s}\left(\mu_{R}^{2}\right)}{\pi}+\left[16.110+8.847 \log \frac{\mu_{R}^{2}}{M_{b}^{2}}+1.791 \log ^{2} \frac{\mu_{R}^{2}}{M_{b}^{2}}\right.\right. \\
& \left.-N_{F}\left(1.041+0.361 \log \frac{\mu_{R}^{2}}{M_{b}^{2}}+0.0833 \log ^{2} \frac{\mu_{R}^{2}}{M_{b}^{2}}\right)+\frac{4}{3} \sum_{1 \leq i \leq N_{F}} \Delta\left(\frac{M_{i}}{M_{b}}\right)\right]\left(\frac{\alpha_{s}\left(\mu_{R}^{2}\right)}{\pi}\right)^{2} \\
& +\left[239.297+129.420 \log \frac{\mu_{R}^{2}}{M_{b}^{2}}+32.105 \log ^{2} \frac{\mu_{R}^{2}}{M_{b}^{2}}+3.683 \log ^{3} \frac{\mu_{R}^{2}}{M_{b}^{2}}-N_{F}(29.701\right.
\end{aligned}
$$




$$
\begin{aligned}
& \left.+15.371 \log \frac{\mu_{R}^{2}}{M_{b}^{2}}+3.053 \log ^{2} \frac{\mu_{R}^{2}}{M_{b}^{2}}+0.370 \log ^{3} \frac{\mu_{R}^{2}}{M_{b}^{2}}\right)+N_{F}^{2}\left(0.653+0.320 \log \frac{\mu_{R}^{2}}{M_{b}^{2}}\right. \\
& \left.\left.\left.+0.0602 \log ^{2} \frac{\mu_{R}^{2}}{M_{b}^{2}}+0.00926 \log ^{3} \frac{\mu_{R}^{2}}{M_{b}^{2}}\right)\right]\left(\frac{\alpha_{s}\left(\mu_{R}^{2}\right)}{\pi}\right)^{3}\right\}+\mathcal{O}\left(\alpha_{s}^{4}\right)
\end{aligned}
$$

where $N_{F}=4$ for the bottom quark and the strong coupling $\alpha_{s}$ evolves with 5 active flavours. The mass-dependent term involving the light flavours can (for $0 \leq x \leq 1$ ) be approximated by

$$
\Delta(x)=\frac{\pi^{2}}{8} x-0.579 x^{2}+0.230 x^{3}
$$

The charm pole mass $M_{c}$ is then determined by the renormalon-free relation [135]

$$
M_{c}=M_{b}-3.41 \mathrm{GeV}
$$

from the bottom pole mass $M_{b}$. The strong coupling $\alpha_{s}$ is fixed by the input value at the $Z$-boson mass scale

$$
\alpha_{s}\left(M_{Z}\right)=0.118 \pm 0.0015
$$

The lepton as well as $W, Z$-boson masses and widths are chosen according to their PDG values [136]

$$
\begin{aligned}
m_{e} & =(0.510998928 \pm 0.000000011) \mathrm{MeV} & \\
m_{\mu} & =(105.6583715 \pm 0.0000035) \mathrm{MeV} & \\
m_{\tau}=(1776.82 \pm 0.16) \mathrm{MeV} & & \\
M_{W} & =(80.385 \pm 0.015) \mathrm{GeV} & \Gamma_{W}=(2.085 \pm 0.042) \mathrm{GeV} \\
M_{Z} & =(91.1876 \pm 0.0021) \mathrm{GeV} & \Gamma_{Z}=(2.4952 \pm 0.0023) \mathrm{GeV}
\end{aligned}
$$

The complex pole masses of the $W$ and $Z$ boson can be obtained from the relations

$$
M_{V}^{\text {pole }}-i \Gamma_{V}^{\text {pole }}=\frac{M_{V}-i \Gamma_{V}}{\sqrt{1+\Gamma_{V}^{2} / M_{V}^{2}}} \quad(V=W, Z)
$$

These are used for the predictions of the Higgs boson decay widths into four fermions according to Ref. [63]. Moreover, the Fermi constant has been chosen as [136]

$$
G_{F}=1.1663787(6) \cdot 10^{-5} \mathrm{GeV}^{-2}
$$

The total parametric uncertainty for each branching ratio has been derived from a quadratic sum of the individual impacts of the input parameters on the decay modes.

The theoretical uncertainties from missing higher orders in the perturbative expansion are summarized in Table 5 as discussed before for the individual partial decay processes. In order to be conservative the total parametric uncertainties are added linearly to the theoretical uncertainties. The final result for the branching ratios is shown in Fig. 16 for the leading Higgs decay modes with branching ratio larger than $10^{-4}$ within the Higgs-mass range between 120 and $130 \mathrm{GeV}$. They have been obtained with Prophecy4F [63] for the decays $H \rightarrow W W, Z Z$ and HDECAY [54] for the other decay modes. The bands represent the total uncertainties of the individual branching ratios. For a Higgs mass $M_{H}=125 \mathrm{GeV}$ the total uncertainty of the leading decay mode $H \rightarrow b \bar{b}$ amounts to less than $2 \%$, since the bulk of it cancels out within the branching ratio. The uncertainty of $\Gamma(H \rightarrow b \bar{b})$, however, generates a significant increase of the uncertainties for the subleading decay modes. The total uncertainties of $B R(H \rightarrow W W / Z Z)$ and $B R\left(H \rightarrow \tau^{+} \tau^{-} / \mu^{+} \mu^{-}\right)$amount to $\sim 2 \%$, while the uncertainties of $B R(H \rightarrow g g)$ and $B R(H \rightarrow c \bar{c})$ range at $\sim 6-7 \%$, of $B R(H \rightarrow \gamma \gamma)$ at $\sim 3 \%$ and of $B R(H \rightarrow Z \gamma)$ at $\sim 7 \%$. The total decay width of $\sim 4.1 \mathrm{MeV}$ can be predicted with $\sim 2 \%$ total uncertainty. These results constitute a basic ingredient of the corresponding LHC analyses. 


\begin{tabular}{llll}
\hline Partial width & QCD & electroweak & total \\
\hline$H \rightarrow b \bar{b} / c \bar{c}$ & $\sim 0.2 \%$ & $\sim 0.5 \%$ & $\sim \pm 0.5 \%$ \\
$H \rightarrow \tau^{+} \tau^{-} / \mu^{+} \mu^{-}$ & & $\sim 0.5 \%$ & $\sim \pm 0.5 \%$ \\
$H \rightarrow g g$ & $\sim 3 \%$ & $\sim 1 \%$ & $\sim \pm 3.2 \%$ \\
$H \rightarrow \gamma \gamma$ & $<1 \%$ & $<1 \%$ & $\sim \pm 1 \%$ \\
$H \rightarrow Z \gamma$ & $<1 \%$ & $\sim 5 \%$ & $\sim \pm 5 \%$ \\
$H \rightarrow W W / Z Z \rightarrow 4 f$ & $<0.5 \%$ & $\sim 0.5 \%$ & $\sim \pm 0.5 \%$ \\
\hline
\end{tabular}

Table 5: Estimated theoretical uncertainties from missing higher orders in the intermediate Higgs-mass range. From Ref. [51] (page 22).

\subsubsection{Minimal supersymmetric extension}

The final results for the MSSM Higgs branching ratios have been obtained with the public codes FEYNHiggs [121] and HDECAY [54] using the SM input parameters of the previous section. Fig. 17 ] shows the neutral Higgs branching ratios for two values of $\operatorname{tg} \beta$ within the $m_{h}^{\text {mod }}+$ benchmark scenario [137] that is defined as

$$
\begin{aligned}
& M_{S U S Y}=1000 \mathrm{GeV}, \mu=M_{2}=200 \mathrm{GeV}, A_{b}=A_{\tau}=A_{t}, m_{\tilde{g}}=1500 \mathrm{GeV}, M_{\tilde{l}_{3}}=1000 \mathrm{GeV}, \\
& X_{t}^{O S}=1.5 M_{S U S Y}\left(\text { FD calculation), } X_{t}^{\overline{\mathrm{MS}}}=1.6 M_{S U S Y}(\mathrm{RG} \text { calculation })\right.
\end{aligned}
$$

The kinks visible in these plots are due to the opening of new decay modes according to the SM and SUSY-particle masses of the final-state particles. Fig. 18 displays the corresponding charged Higgs branching ratios within the $m_{h}^{\text {mod+ }}$ benchmark scenario [137] for two values of $\operatorname{tg} \beta$. The related uncertainties are not shown in the plots.

\section{Higgs-Boson Production}

\subsection{Gluon fusion: $g g \rightarrow H$}

\subsubsection{Standard Model}

The gluon-fusion mechanism [16]

$$
p p \rightarrow g g \rightarrow H
$$

dominates Higgs-boson production at the LHC in the entire relevant Higgs mass range. The gluon coupling to the Higgs boson in the SM is mediated by triangular top- and bottom-quark loops, see Fig. 19. Since the Yukawa coupling of the Higgs particle grows with the quark mass, the form factor reaches a constant value for large loop quark masses. If the masses of heavier quarks beyond the third generation are fully generated by the Higgs mechanism, these particles would add the same amount to the form factor as the top quark in the asymptotic heavy quark limit. Thus gluon fusion can serve as a counter of the number of heavy quarks, the masses of which are generated by the conventional Higgs mechanism. On the other hand within the three-generation SM gluon fusion will allow to measure the top quark Yukawa coupling. This, however, requires a precise knowledge of the cross section within the SM with three generations of quarks.

The partonic cross section can be derived from the gluonic width of the Higgs boson at lowest order [16],

$$
\hat{\sigma}_{L O}(g g \rightarrow H)=\sigma_{0} \delta(1-z)
$$




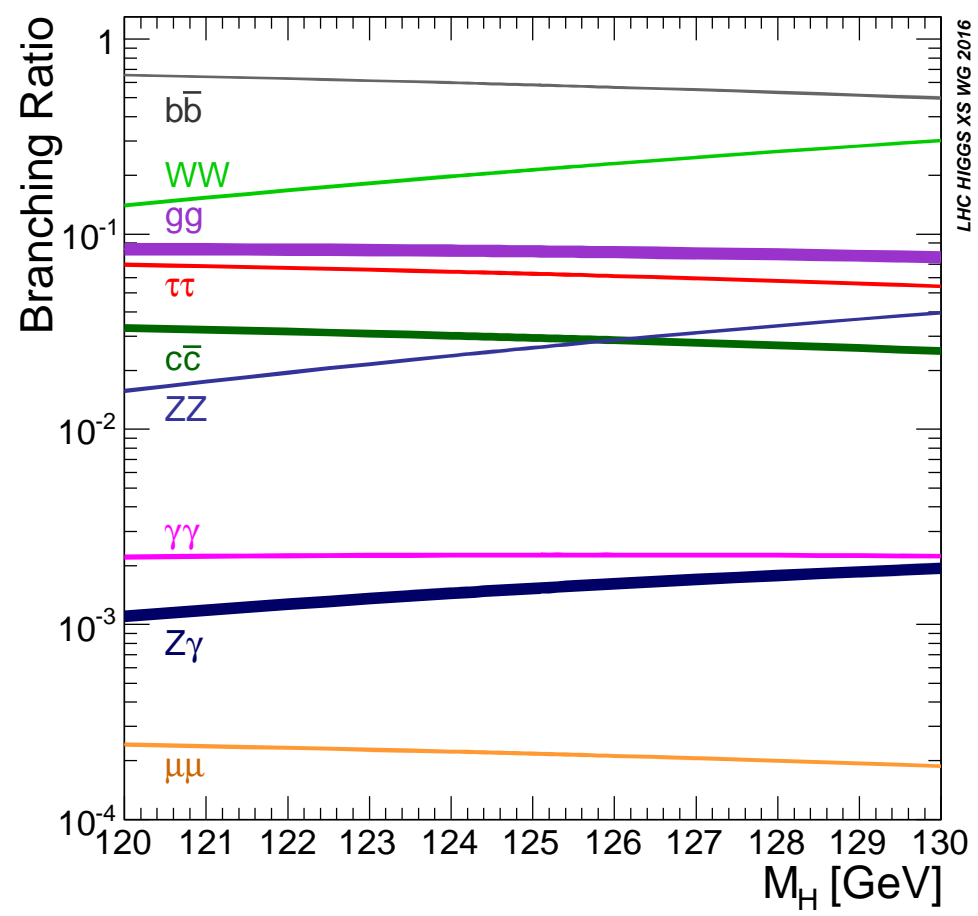

Figure 16: Higgs branching ratios and their uncertainties for the mass range around 125 GeV. From Ref. [51] (page 24).

$$
\sigma_{0}=\frac{\pi^{2}}{8 M_{H}^{3}} \Gamma_{L O}(H \rightarrow g g)=\frac{G_{F} \alpha_{s}^{2}\left(\mu_{R}\right)}{288 \sqrt{2} \pi}\left|\sum_{Q} A_{Q}^{H}\left(\tau_{Q}\right)\right|^{2}
$$

with the scaling variables defined as $z=M_{H}^{2} / \hat{s}, \tau_{Q}=4 M_{Q}^{2} / M_{H}^{2}$. The variable $\hat{s}$ denotes the partonic c.m. energy squared and $\mu_{R}$ the renormalization scale. The amplitudes $A_{Q}^{H}\left(\tau_{Q}\right)$ are given in Eq. (50).

In the narrow-width approximation the hadronic cross section can be cast into the form [16]

$$
\sigma_{L O}(p p \rightarrow H)=\sigma_{0} \tau_{H} \frac{d \mathcal{L}^{g g}}{d \tau_{H}}
$$

with the gluon luminosity

$$
\frac{d \mathcal{L}^{g g}}{d \tau}=\int_{\tau}^{1} \frac{d x}{x} g\left(x, \mu_{F}^{2}\right) g\left(\tau / x, \mu_{F}^{2}\right)
$$

at the factorization scale $\mu_{F}$, and the scaling variable is defined, in analogy to the Drell-Yan process, as $\tau_{H}=M_{H}^{2} / s$, with $s$ specifying the total hadronic c.m. energy squared. The bottom-quark contributions interfere destructively with the top loop and decrease the cross section by about 10\% at LO.

QCD corrections. In the past the (two-loop) NLO QCD corrections to the gluon-fusion cross section, Fig. 20, have been calculated including the full mass dependences [68, 108, 70, 138, 139, 140]. They consist of virtual corrections to the basic $g g \rightarrow H$ process and real corrections due to the associated production of the Higgs boson with massless partons,

$$
g g \rightarrow H g \quad \text { and } \quad g q \rightarrow H q, q \bar{q} \rightarrow H g
$$

These subprocesses contribute to the Higgs production at $\mathcal{O}\left(\alpha_{s}^{3}\right)$. The virtual corrections rescale the lowest-order fusion cross section with a coefficient depending only on the ratios of the Higgs and quark 

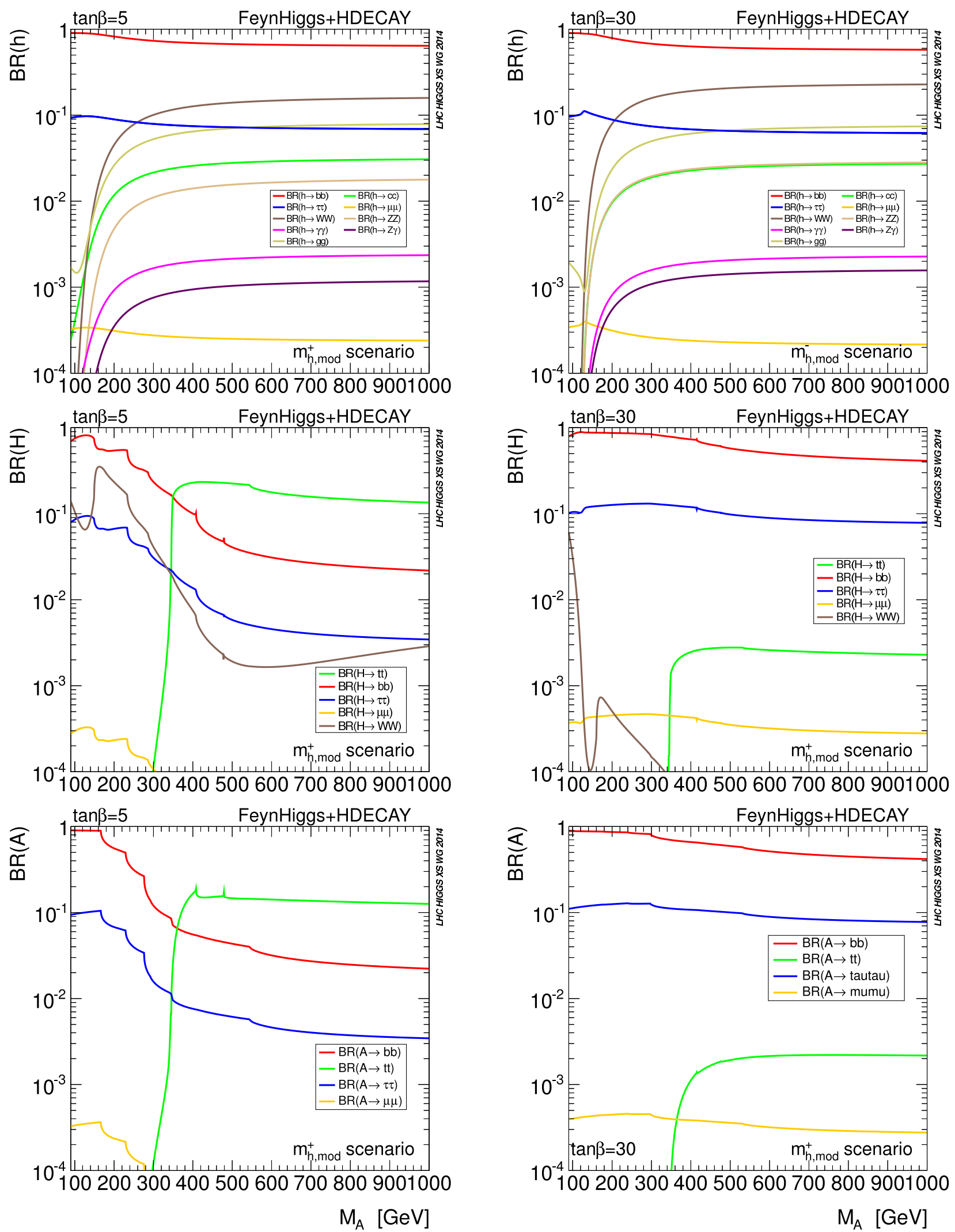

Figure 17: Neutral MSSM Higgs branching ratios as functions of the corresponding Higgs mass within the $m_{h}^{\text {mod }}$ scenario [137] for two values of tg $\beta$ obtained by a combination of FEYNHIGGS [121] and HDECAY [54]. From Ref. [51]. 

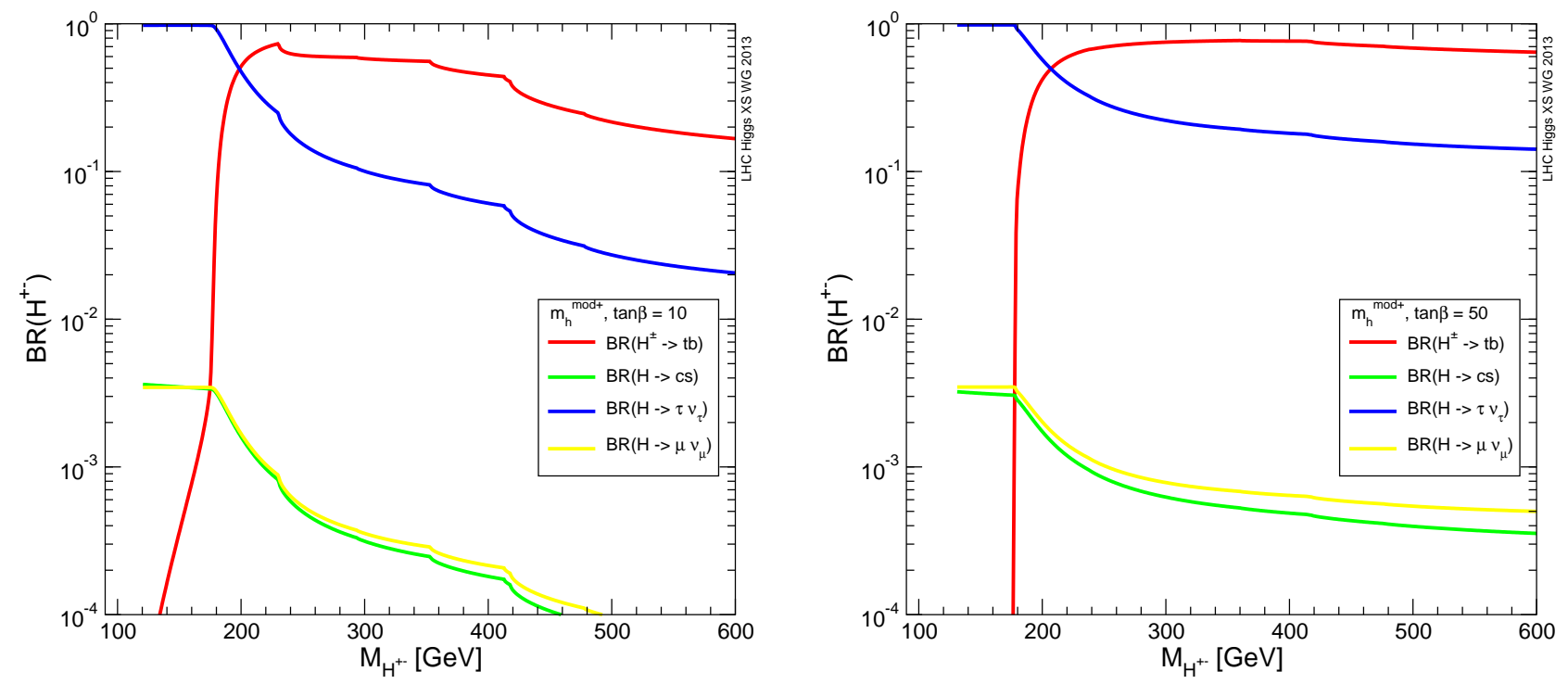

Figure 18: Charged MSSM Higgs branching ratios as functions of the charged Higgs mass within the $m_{h}^{\text {mod }+}$ scenario [137] for two values of tg $\beta$ obtained by a combination of FEYNHigGs [121] and HDECAY [54]. From Ref. [66].

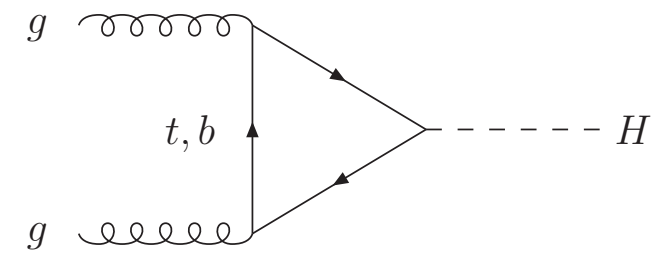

Figure 19: Diagrams contributing to $g g \rightarrow H$ at lowest order.

masses. Gluon radiation leads to two-parton final states with invariant energy $\hat{s} \geq M_{H}^{2}$ in the $g g, g q$ and $q \bar{q}$ channels at NLO. In general the hadronic cross section can be split into seven parts [68, 108, 170, 139, 140],

$$
\sigma(p p \rightarrow H+X)=\sigma_{0}\left[1+C \frac{\alpha_{s}}{\pi}\right] \tau_{H} \frac{d \mathcal{L}^{g g}}{d \tau_{H}}+\Delta \sigma_{g g}+\Delta \sigma_{g q}+\Delta \sigma_{q \bar{q}}+\Delta \sigma_{q q}+\Delta \sigma_{q q^{\prime}}
$$

where the finite parts of virtual corrections $C$ and the real corrections $\Delta \sigma_{g g}, \Delta \sigma_{g q}$ and $\Delta \sigma_{q \bar{q}}$ (sameflavour quark-antiquark initial states) start to contribute at NLO, while $\Delta \sigma_{q q}$ (same-flavour quark-quark and antiquark-antiquark initial states) and $\Delta \sigma_{q q^{\prime}}$ (different-flavour quark and antiquark initial states) appear for the first time at NNLO. The renormalization scale $\mu_{R}$ of $\alpha_{s}$ and the factorization scale $\mu_{F}$ of the parton densities are fixed properly, in general at $\mu_{R}=\mu_{F}=M_{H} / 2$. The quark-loop mass has been identified with the pole mass $M_{Q}$, while the QCD coupling $\alpha_{s}$ and the parton density functions are defined in the $\overline{\mathrm{MS}}$ scheme with five active flavours.

We define the NLO $K$ factor as the ratio

$$
K_{N L O}=\frac{\sigma_{N L O}}{\sigma_{L O}}
$$



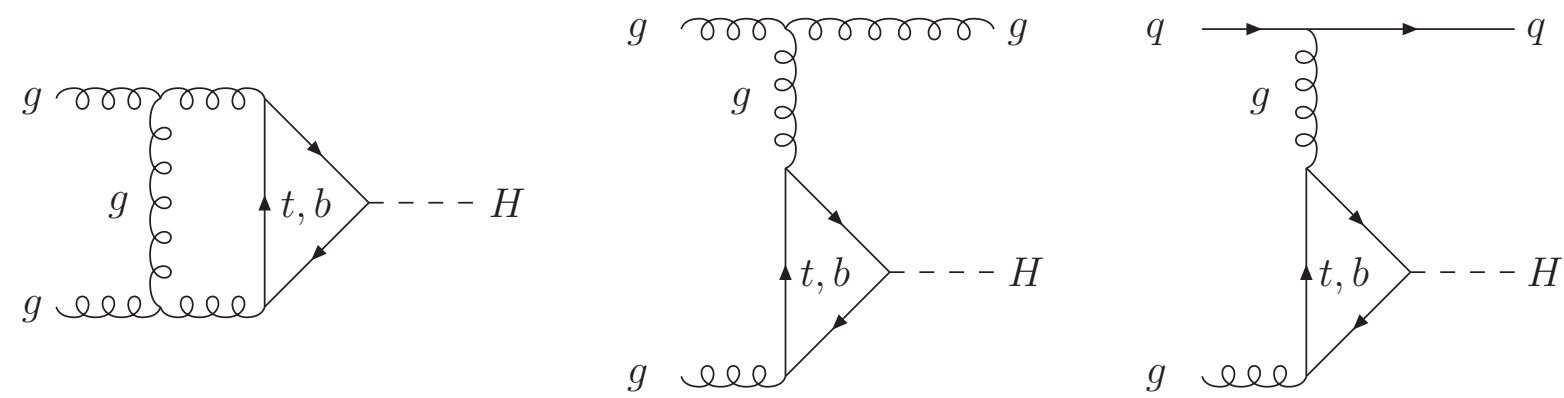

Figure 20: Typical diagrams contributing to the virtual and real $Q C D$ corrections to $g g \rightarrow H$ at $N L O$.

The NLO corrections are positive and large, increasing the gluon-fusion cross section at the LHC by about $60-90 \%$. The QCD corrections to the bottom-quark contributions are significantly smaller if the bottom mass is used in terms of the pole mass or the $\overline{\mathrm{MS}}$ mass at the scale of the bottom mass. This choice is motivated by the numerical cancellation of squared and single logarithms of the relative QCD corrections at NLO [68]. This feature modifies the destructive bottom-quark contribution to a reduction of the cross section by about $6 \%$ at NLO. Comparing the exact mass-dependent results with the expressions in the heavy-quark limit, it turns out that this asymptotic $K$ factor provides an excellent approximation even for Higgs masses above the top-decay threshold8. We explicitly define the approximation by

$$
\begin{aligned}
\sigma_{a p p} & =K_{N L O}^{t}(\infty) \times \sigma_{L O}\left(\tau_{t}, \tau_{b}, \tau_{c}\right) \\
K_{N L O}^{t}(\infty) & =\lim _{M_{t} \rightarrow \infty} K_{N L O}
\end{aligned}
$$

where we neglect the $b$ quark contribution in $K_{N L O}^{t}(\infty)$, while the leading order cross section $\sigma_{L O}$ includes the full $t, b, c$ quark mass dependence. The comparison with the full massive NLO result is presented in Fig. 21. The solid line corresponds to the exact cross section and the broken line to the approximate one. For Higgs masses below $\sim 1 \mathrm{TeV}$, the deviations of the asymptotic approximation from the full NLO result are less than $15 \%$, whereas for $M_{H}=125 \mathrm{GeV}$ they amount to $\sim 5 \%$, if the full LO cross section is multiplied by the approximate K-factor. This property of the NLO corrections suggests this to be true also at higher orders, since it is a consequence of the dominating soft and collinear gluon effects in the QCD corrections.

Within the heavy top-quark limit the NNLO [142] and $\mathrm{N}^{3} \mathrm{LO}$ [143, 144] QCD corrections have been calculated. The NNLO contributions increase the production cross section by about $20 \%$ beyond $\mathrm{NLO}$, while the $\mathrm{N}^{3} \mathrm{LO}$ corrections range at the level of a few per-cent. These results indicate that the gluon-fusion cross section is under theoretical control despite the large size of the NLO corrections. This is further corroborated by the results obtained by a soft and collinear gluon resummation on top of the $\mathrm{N}^{3} \mathrm{LO}$ result. This approach resums the dominant factorizing contributions from soft and collinear gluon effects up to all perturbative orders. The soft corrections provide the leading ones close to the production threshold, while collinear gluon effects are of subleading order. Both can be treated systematically. The soft and collinear contributions provide a reasonable approximation of the full fixed-order results and thus a reliable estimate of missing higher-order effects beyond the fixed-order corrections. This resummation has been performed at the NNLL level [145] and the $\mathrm{N}^{3}$ LL level [146] in the heavy top-quark limit. The sizes of the different logarithmic orders follow roughly the pattern of the corresponding fixed-order corrections. Quite recently also finite top-mass effects have been included in the resummation framework [147] at the NLL order, where they are known exactly. Resummation effects beyond $\mathrm{N}^{3} \mathrm{LO}$ yield only a per-cent increase of the cross section for the central scale choice. However, they provide an approximation of effects beyond $\mathrm{N}^{3} \mathrm{LO}$ and contribute to a sophisticated

\footnotetext{
${ }^{8}$ Large Higgs masses are still relevant for off-shell Higgs bosons.
} 


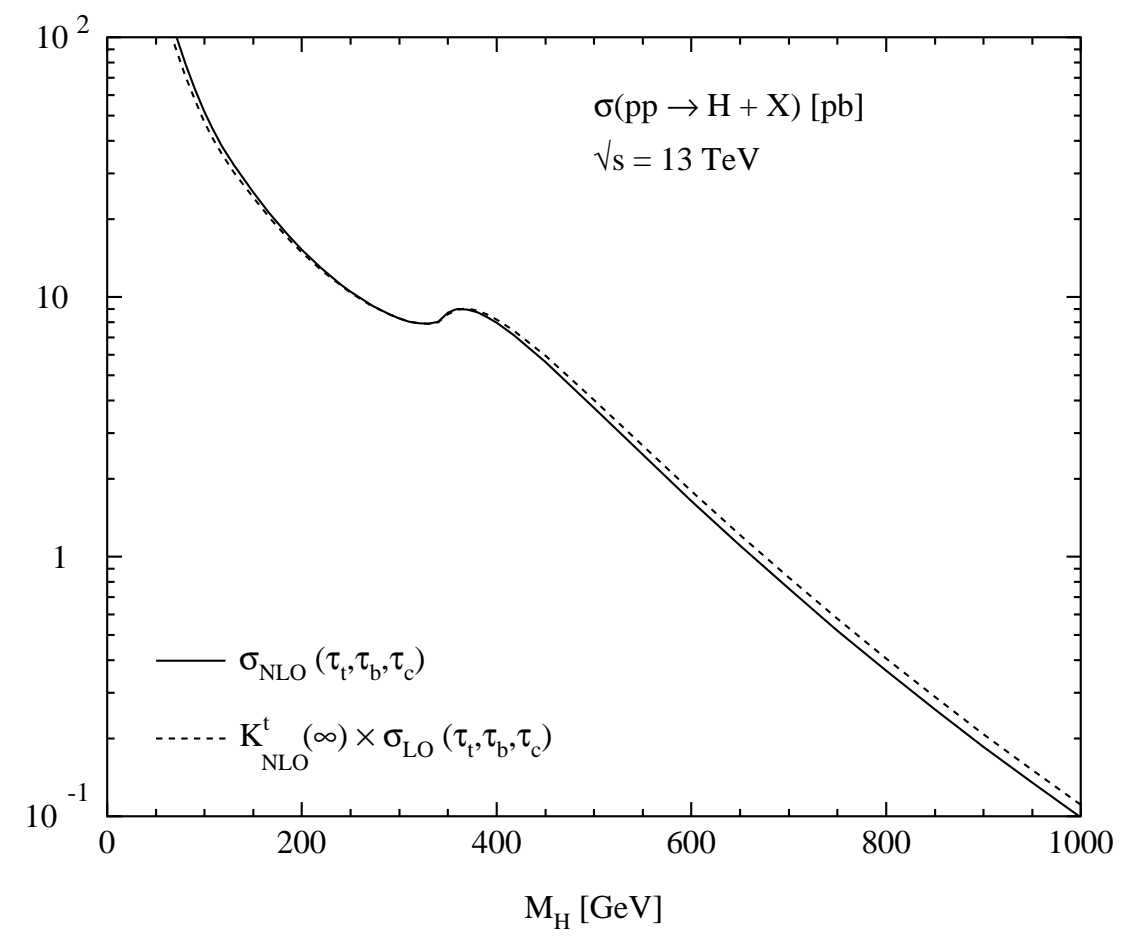

Figure 21: Comparison of the exact and approximate $N L O$ cross section $\sigma(p p \rightarrow H+X)$ at the LHC with c.m. energy $\sqrt{s}=13 \mathrm{TeV}$. The solid line shows the exact cross section including the full $t, b, c$ quark mass dependence and the dashed line corresponds to the approximation defined in Eq. 164). The renormalization and factorization scales have been identified with half of the Higgs mass, $\mu_{R}=\mu_{F}=$ $M_{H} / 2$ and the PDF 4 LHC15 NLO parton densities [141] with NLO strong coupling $\left(\alpha_{s}\left(M_{Z}\right)=0.118\right)$ have been adopted. The top mass has been chosen as $M_{t}=172.5 \mathrm{GeV}$, the bottom mass as $M_{b}=4.84$ GeV and the charm mass as $M_{c}=1.43 \mathrm{GeV}$.

estimate of the residual uncertainties by elaborating on the uncertainties due to the matching to the fixed-order expression.

Electroweak corrections. The electroweak corrections to the gluon-fusion cross section have been computed approximately first. The leading top mass corrections of $\mathcal{O}\left(G_{F} M_{t}^{2}\right)$ coincide with the corrections to the gluonic decay mode of Eq. (60) and are thus small [49, 78]. This calculation has been refined by the determination of the NLO electroweak corrections due to light-fermion loops [79] and finally by the full numerical integration of the exact NLO corrections to the top- and $W, Z$-induced electroweak corrections [80]. The electroweak corrections coincide with the ones to the $H \rightarrow g g$ decay. The NLO electroweak corrections have been extended by a calculation of the mixed QCD-electroweak corrections in the limit $M_{H}^{2} \ll M_{W}^{2}$ [148] which can be attributed to the corrections of the effective $\mathrm{Hgg}$ coupling. However, it is unclear how reliable this approximation is in practice. Due to the dominance of soft and collinear gluon effects the bulk of the electroweak corrections will factorize from the pure QCD corrections. In the following the electroweak corrections will be combined with the QCD corrections in factorized form.

Total cross section. Theoretical uncertainties in the prediction of the Higgs cross section originate from three major sources, the dependence of the cross section on the parton densities, the unknown corrections beyond $\mathrm{N}^{3} \mathrm{LO}$ and the parametric uncertainties originating from the input value of the strong coupling $\alpha_{s}$ and to a lesser extent of the top and bottom quark masses. The missing quark-mass effects beyond NLO have been estimated as less than $1 \%$ by an explicit large top-mass expansion of the 
NNLO corrections beyond the heavy top-quark limit [149]. The total uncertainty of the prediction for the total gluon-fusion cross sections has been estimated as $4 \%$ and can dominantly be traced back to the renormalization- and factorization-scale dependence as well as the PDF $+\alpha_{s}$ uncertainties [51]. The renormalization- and factorization-scale dependence is depicted in Fig. 22 for the known perturbative orders in the heavy top-quark limit. A significant reduction from $50-100 \%$ to the few-per-cent level is visible from LO to $\mathrm{N}^{3} \mathrm{LO}$. The results for the cross section are contained in the public codes HigLU [150] up to NNLO as well as SusHi [151, iHix [152] and GGHiggs [153] up to $\mathrm{N}^{3} \mathrm{LO}$. The soft/collineargluon-resummed results can be obtained with the code TroLL for corrections beyond $\mathrm{N}^{3} \mathrm{LO}$ [154].

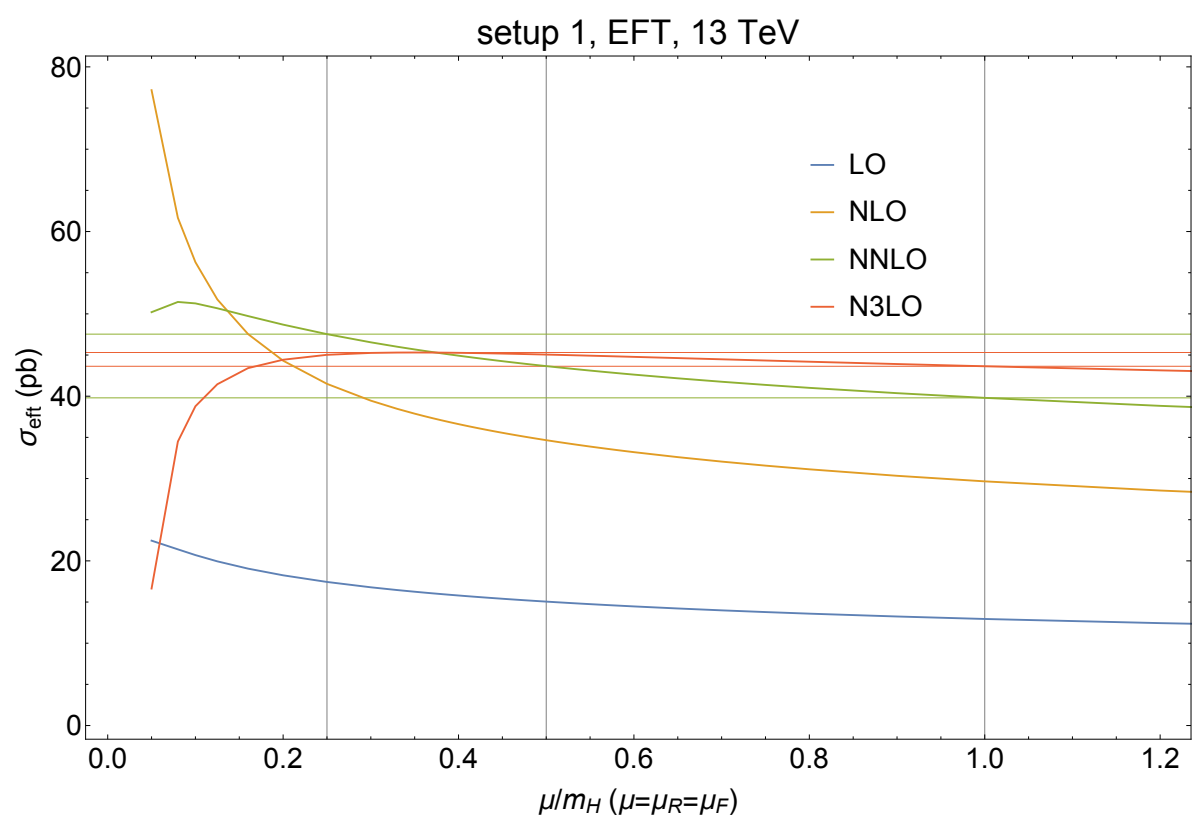

Figure 22: Renormalization- and factorization-scale dependence of the gluon fusion cross section as a function of the identified scales in units of the Higgs boson mass at different perturbative orders for a Higgs mass $M_{H}=125 \mathrm{GeV}$ at the $\mathrm{LHC}$ with $\sqrt{\mathrm{s}}=13 \mathrm{TeV}$ within the heavy-top-quark limit. From Ref. 144.

Transverse-momentum distribution. Apart from the total cross section also distributions are relevant for analyzing the Higgs properties. Of particular interest in this context is the transversemomentum distribution of the Higgs boson that arises due to the additional radiation of gluons. The LO contributions are part of the real corrections to the gluon-fusion cross section at NLO. The first results were obtained a long time ago [155] that include the full quark-mass dependence at LO. The NLO QCD corrections were calculated in the heavy top-quark limit [156] that is expected to be reliable up to $p_{T}$-values below the $t \bar{t}$ threshold as can be inferred from a large top-mass expansion [157]. Recently a more rigorous inclusion of finite top-mass effects at NLO has been studied by using exact results partially [158]. These calculations are extended by the recent derivation of the NNLO QCD corrections in the heavy-top limit 159. These predictions have been matched to a soft-gluon resummed expression that provides a regular prediction for small values of $p_{T}$ thus removing the spurious singularities in $\log \left(M_{H}^{2} / p_{T}^{2}\right)$ in fixed-order expressions [160, 161]. The basic structure of matched fixed-order and resummed expression can be sketched as

$$
\frac{d \sigma}{d p_{T}^{2}}=\frac{d \sigma^{(r e s)}}{d p_{T}^{2}}-\left[\frac{d \sigma^{(r e s)}}{d p_{T}^{2}}\right]_{f . o .}+\frac{d \sigma^{(f . o .)}}{d p_{T}^{2}}
$$


where $d \sigma^{(r e s)}$ denotes the resummed and $d \sigma^{(f . o .)}$ the fixed-order differential cross section. The resummed part is given by

$$
\frac{d \sigma^{(r e s)}}{d p_{T}^{2}}=\sum_{a b=g g, g q, q \bar{q}} \int_{0}^{1} d x_{1} \int_{0}^{1} d x_{2} f_{a}\left(x_{1}, \mu_{F}^{2}\right) f_{b}\left(x_{2}, \mu_{F}^{2}\right) \frac{d \hat{\sigma}_{a b}^{(r e s)}}{d p_{T}^{2}}
$$

with the partonic cross section kernel resummed in impact-parameter space [160, 161],

$$
\frac{d \hat{\sigma}^{(r e s)}}{d p_{T}^{2}}=z \int_{0}^{\infty} d b \frac{b}{2} J_{0}\left(b p_{T}\right) \mathcal{W}_{a b}\left(b, M_{H}, \hat{s}=M_{H}^{2} / z ; \alpha_{s}\left(\mu_{R}^{2}\right), \mu_{R}^{2}, \mu_{F}^{2}\right)
$$

where $\mu_{R}\left(\mu_{F}\right)$ denotes the renormalization (factorization) scale and $J_{0}(x)$ the 0th-order Bessel function. Introducing Mellin moments $f_{N}=\int_{0}^{1} d z z^{N-1} f(z)$ the resummed factor $\mathcal{W}_{a b, N}$ factorizes into a (perturbative) hard part $\mathcal{H}_{\mathcal{N}}$ and an exponential factor,

$$
\mathcal{W}_{a b, N}=\mathcal{H}_{N} \exp \mathcal{G}_{N}
$$

where the functional dependences have been suppressed. The exponent $\mathcal{G}_{N}$ can be expanded as

$$
\mathcal{G}_{N}=L g^{(1)}\left(\alpha_{s} L\right)+g_{N}^{(2)}\left(\alpha_{s} L\right)+\sum_{n=3}^{\infty}\left(\frac{\alpha_{s}}{\pi}\right)^{n-2} g_{N}^{(n)}\left(\alpha_{s} L\right)
$$

Again functional dependences on the renormalization and resummation scales have been suppressed. The basic ingredient of the resummation formula is the large logarithmic expansion parameter

$$
L=\log \frac{Q^{2} b^{2}}{b_{0}^{2}}
$$

with $b_{0}=2 e^{-\gamma_{E}}\left(\gamma_{E}=0.5772 \ldots\right.$ is the Euler number $)$ and $Q$ denoting the resummation scale. In order to impose the unitarity constraint that the integration over the transverse momentum reproduces the known fixed-order cross section the logarithmic parameter can be shifted to [161]

$$
L \rightarrow \bar{L}=\log \left(\frac{Q^{2} b^{2}}{b_{0}^{2}}+1\right)
$$

without modifying the limit of large $L$ (i.e. large $b$ ) but introducing a damping of effects for small impact parameters $b$ that are not covered by the resummation. The expressions for the terms $g_{N}^{(i)}$ can be found in [161]. The consistent matching between the resummed and fixed order expressions requires the subtraction of the fixed-order expansion of the resummed expression as shown by the second term in Eq. (165). This matching has been performed at NNLL+NNLO level [161]. In addition finite quarkmass effects have been included in the resummed expressions at NLL+NLO, too [162, 163] 10. The latter step requires to use different matching scales $Q$ for the pure top-induced contributions and those that involve bottom loops. The resummation work has been extended to the case of the $p_{T}$ distribution for fixed rapidity at the NNLL+NNLO level [164]. During the last years the full NLO expressions have been implemented in the Powheg box [165] providing in this way a reliable NLO event generator [166]. A second matching to parton showers has been performed by combining SusHi [151] and the MC@NLO [167] framework in the code AMCSUsHi [168] at NLO accuracy, too.

\footnotetext{
${ }^{9}$ Here NNLO refers to the corresponding total cross section, while it is NLO for the $p_{T}$ distribution at large $p_{T}$ values.

${ }^{10} \mathrm{NLO}$ refers to LO-mass effects on the $p_{T}$ distribution at large $p_{T}$ values.
} 


\subsubsection{Minimal supersymmetric extension}

The gluon-fusion mechanism [16]

$$
p p \rightarrow g g \rightarrow \Phi
$$

dominates the neutral MSSM Higgs boson production at the LHC in the phenomenologically relevant Higgs mass ranges for small and moderate values of $\operatorname{tg} \beta$. Only for large $\operatorname{tg} \beta$ can the associated $\Phi b \bar{b}$ production channel develop a larger cross section due to the enhanced Higgs couplings to bottom quarks [19, 169]. Analogous to the gluonic decay modes, the gluon coupling to the neutral Higgs bosons in the MSSM is built up by loops involving top and bottom quarks as well as squarks, see Fig. 23.

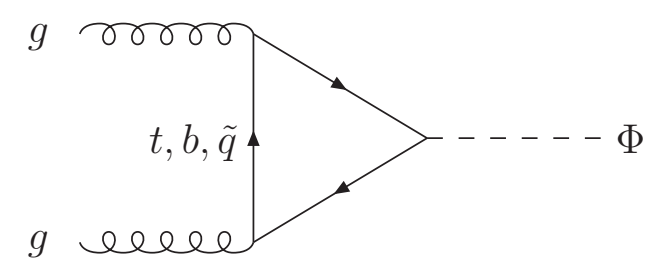

Figure 23: Typical diagram contributing to $g g \rightarrow \Phi$ at lowest order.

The partonic cross sections can be obtained from the gluonic widths of the Higgs bosons at lowest order [68, 81]:

$$
\begin{aligned}
\hat{\sigma}_{L O}^{\Phi}(g g \rightarrow \Phi) & =\sigma_{0}^{\Phi} \delta(1-z) \\
\sigma_{0}^{\Phi} & =\frac{\pi^{2}}{8 M_{\Phi}^{3}} \Gamma_{L O}(\Phi \rightarrow g g) \\
\sigma_{0}^{h / H} & =\frac{G_{F} \alpha_{s}^{2}(\mu)}{288 \sqrt{2} \pi}\left|\sum_{Q} g_{Q}^{h / H} A_{Q}^{h / H}\left(\tau_{Q}\right)+\sum_{\widetilde{Q}} g_{\widetilde{Q}}^{h / H} A_{\widetilde{Q}}^{h / H}\left(\tau_{\widetilde{Q}}\right)\right|^{2} \\
\sigma_{0}^{A} & =\frac{G_{F} \alpha_{s}^{2}(\mu)}{128 \sqrt{2} \pi}\left|\sum_{Q} g_{Q}^{A} A_{Q}^{A}\left(\tau_{Q}\right)\right|^{2}
\end{aligned}
$$

where the scaling variables are defined as $z=M_{\Phi}^{2} / \hat{s}, \tau_{i}=4 M_{i}^{2} / M_{\Phi}^{2}(i=Q, \widetilde{Q})$, and $\hat{s}$ denotes the partonic c.m. energy squared. The amplitudes $A_{Q, \widetilde{Q}}^{\Phi}\left(\tau_{Q, \widetilde{Q}}\right)$ are defined in Eqs. (61), 65), and the MSSM couplings $g_{Q}^{\Phi}, g_{\widetilde{Q}}^{\Phi}$ can be found in Tables 1 and 2. In the narrow-width approximation the hadronic cross sections are given by

$$
\sigma_{L O}(p p \rightarrow \Phi)=\sigma_{0}^{\Phi} \tau_{\Phi} \frac{d \mathcal{L}^{g g}}{d \tau_{\Phi}}
$$

with the gluon luminosity defined in Eq. (161) and the scaling variables $\tau_{\Phi}=M_{\Phi}^{2} / s$ where $s$ specifies the total hadronic c.m. energy squared. For small $\operatorname{tg} \beta$ the top loop contribution is dominant, while for large values of $\operatorname{tg} \beta$ the bottom quark contribution is strongly enhanced. If the squark masses are less than $\sim 400 \mathrm{GeV}$, their contribution is significant, and for squark masses beyond $\sim 500 \mathrm{GeV}$ they can safely be neglected [81].

QCD corrections. In the past the full two-loop QCD corrections to the quark and squark loops of the gluon-fusion cross section were calculated [68, 108, 83, 89]. In complete analogy to the SM case they consist of virtual corrections to the basic $g g \rightarrow \Phi$ process and real corrections due to the associated 
production of the Higgs bosons with massless partons. Thus the contributions to the final result for the hadronic cross section can in complete analogy to the SM case be classified as

$$
\sigma(p p \rightarrow \Phi+X)=\sigma_{0}^{\Phi}\left[1+C^{\Phi} \frac{\alpha_{s}}{\pi}\right] \tau_{\Phi} \frac{d \mathcal{L}^{g g}}{d \tau_{\Phi}}+\Delta \sigma_{g g}^{\Phi}+\Delta \sigma_{g q}^{\Phi}+\Delta \sigma_{q \bar{q}}^{\Phi}+\Delta \sigma_{q q}^{\Phi}+\Delta \sigma_{q q^{\prime}}^{\Phi}
$$

where $\Delta \sigma_{q q}^{\Phi}$ and $\Delta \sigma_{q q^{\prime}}^{\Phi}$ start to contribute at NNLO. The analytic NLO expressions for arbitrary Higgs boson and quark masses are rather involved and can be found in [68, 108]. As in the SM case the (s)quark-loop masses have been identified with the pole masses $M_{Q}\left(M_{\widetilde{Q}}\right)$, while the QCD coupling and PDFs of the proton are defined in the $\overline{\mathrm{MS}}$ scheme with five active flavours. The axial $\gamma_{5}$ coupling can be regularized in the 't Hooft-Veltman scheme [87] or its extension by Larin [88], which preserves the chiral symmetry in the massless quark limit by the addition of supplementary counter terms and fulfills the non-renormalization theorem [95] of the ABJ anomaly [93] at vanishing momentum transfer. The same result can also be obtained with the scheme of Ref. [90] that gives up the cyclicity of the traces involving Clifford matrices.

Similar to the SM the leading terms in the heavy-quark limit provide a reliable approximation for small $\operatorname{tg} \beta$ up to Higgs masses of $\sim 1 \mathrm{TeV}$ with a maximal deviation of $\sim 25 \%$ for $\operatorname{tg} \beta \lesssim 5$ in the intermediate mass range. The squark contributions in the heavy-squark limit coincide with the heavyquark case apart from the mismatch related to the effective Lagrangians of Eqs. (55, 64). The genuine SUSY-QCD corrections are the same as for the gluonic decay width as discussed in Section 2.3.2. They are moderate for small values of $\operatorname{tg} \beta$ [104, 105], but can be large for large $\operatorname{tg} \beta$ due to the contribution of the $\Delta_{b}$ terms and a sizeable remainder beyond these approximate contributions [105].

In the opposite limit, where the Higgs mass is much larger than the quark mass, the analytic results coincide with the SM expressions for both the scalar and pseudoscalar Higgs particles up to NLO at which the results for small quark masses are known 68. This coincidence reflects the restoration of the chiral symmetry in the massless quark limit. The leading logarithmic structure is the same as in the SM case, see Eq. (72). The non-Abelian part of these logarithms has not been resummed so that the uncertainties for large values of $\operatorname{tg} \beta$ are sizeable due to the dominance of the bottom-quark loops involving the large logarithms.

The QCD corrections are positive and large, increasing the MSSM Higgs production cross sections at the LHC by up to about $100 \%$. The effect of the pure squark loops (without gluino exchange) on the scalar Higgs $K$ factors is of moderate size and lead to a maximal modification by about 10\%. Squark mass effects on top of the LO ones can amount to $\sim 20 \%$ for large Higgs masses [83]. For the top-loop contributions alone the $\mathrm{N}^{3} \mathrm{LO}$ corrections can be used consistently. SUSY-electroweak corrections are unknown so far.

Transverse-momentum distribution. The results of the POWHEG implementation of the full LO matrix elements for the transverse-momentum distribution of Ref. [166] and AMCSUsHi [168] can also be used for the MSSM, since they also include variable top and bottom Yukawa couplings. The code of Ref. [166] includes squark-loop contributions in addition. A rigorous comparison between the POWHEG implementation and analytical resummation approaches [162] as well as AMCSUsHi has been performed [163, 51]. This comparison addressed in particular different approaches for the setting of the resummation scale of the bottom-loop contributions. In scenarios where the top loops provide the dominant contribution the differences between the codes are in the range of up to about $50 \%$, while for scenarios with bottom-loop dominance they are larger and can reach 100\% [163, 51]. These differences will only be reduced by calculating the NLO corrections to the transverse-momentum distribution including the full mass dependence. 


\subsection{Vector-boson fusion: $q q \rightarrow q q V^{*} V^{*} \rightarrow q q H$}

\subsubsection{Standard Model}

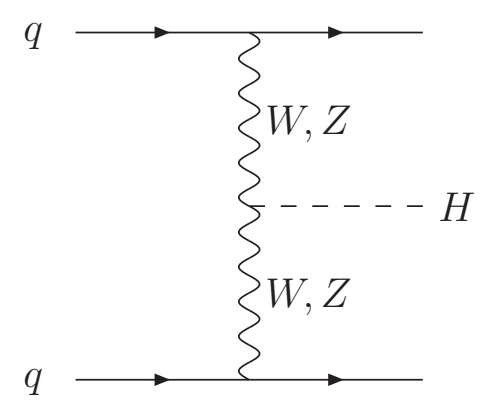

Figure 24: Diagram contributing to $q q \rightarrow q q V^{*} V^{*} \rightarrow q q H$ at lowest order.

At the LHC the second important Higgs production channel is the vector-boson-fusion (VBF) mechanism (see Fig. 24) [17, 170]. For intermediate Higgs masses the vector-boson-fusion cross section is about one order of magnitude smaller than the gluon-fusion one. The cross section can be approximated by the $t$-channel diagrams of the type shown in Fig. 24 within $\sim 1 \%$ accuracy, i.e. without any colour-cross talk between the quark lines, while interference effects for identical quark flavours and $s$-channel contributions are at the per-cent level after subtracting the corresponding Higgs-strahlung component from the $s$-channel contributions [171]. Within the structure-function approach the leading order partonic vector-boson-fusion cross section [17] can be cast into the form $(V=W, Z)$ :

$$
\begin{aligned}
d \sigma_{L O}= & \frac{1}{4} \frac{\sqrt{2} G_{F}^{3} M_{V}^{8} q_{1}^{2} q_{2}^{2}}{\left[q_{1}^{2}-M_{V}^{2}\right]^{2}\left[q_{2}^{2}-M_{V}^{2}\right]^{2}} \\
& \left\{F_{1}\left(x_{1}, \mu_{F}^{2}\right) F_{1}\left(x_{2}, \mu_{F}^{2}\right)\left[2+\frac{\left(q_{1} q_{2}\right)^{2}}{q_{1}^{2} q_{2}^{2}}\right]\right. \\
& +\frac{F_{1}\left(x_{1}, \mu_{F}^{2}\right) F_{2}\left(x_{2}, \mu_{F}^{2}\right)}{P_{2} q_{2}}\left[\frac{\left(P_{2} q_{2}\right)^{2}}{q_{2}^{2}}-M_{P}^{2}+\frac{1}{q_{1}^{2}}\left(P_{2} q_{1}-\frac{P_{2} q_{2}}{q_{2}^{2}} q_{1} q_{2}\right)^{2}\right] \\
& +\frac{F_{2}\left(x_{1}, \mu_{F}^{2}\right) F_{1}\left(x_{2}, \mu_{F}^{2}\right)}{P_{1} q_{1}}\left[\frac{\left(P_{1} q_{1}\right)^{2}}{q_{1}^{2}}-M_{P}^{2}+\frac{1}{q_{2}^{2}}\left(P_{1} q_{2}-\frac{P_{1} q_{1}}{q_{1}^{2}} q_{1} q_{2}\right)^{2}\right] \\
& +\frac{F_{2}\left(x_{1}, \mu_{F}^{2}\right) F_{2}\left(x_{2}, \mu_{F}^{2}\right)}{\left(P_{1} q_{1}\right)\left(P_{2} q_{2}\right)}\left[P_{1} P_{2}-\frac{\left(P_{1} q_{1}\right)\left(P_{2} q_{1}\right)}{q_{1}^{2}}-\frac{\left(P_{2} q_{2}\right)\left(P_{1} q_{2}\right)}{q_{2}^{2}}\right. \\
& \left.+\frac{\left(P_{1} q_{1}\right)\left(P_{2} q_{2}\right)\left(q_{1} q_{2}\right)}{q_{1}^{2} q_{2}^{2}}\right]^{2} \\
& \\
& \left.\frac{F_{3}\left(x_{1}, \mu_{F}^{2}\right) F_{3}\left(x_{2}, \mu_{F}^{2}\right)}{2\left(P_{1} q_{1}\right)\left(P_{2} q_{2}\right)}\left[\left(P_{1} P_{2}\right)\left(q_{1} q_{2}\right)-\left(P_{1} q_{2}\right)\left(P_{2} q_{1}\right)\right]\right\} d x_{1} d x_{2} \frac{d P S_{3}}{\hat{s}}
\end{aligned}
$$

where $d P S_{3}$ denotes the three-particle phase space of the final-state particles, $M_{P}$ the proton mass, $P_{1,2}$ the proton momenta and $q_{1,2}$ the momenta of the virtual vector bosons $V^{*}$. The functions $F_{i}\left(x, \mu_{F}^{2}\right)(i=$ $1,2,3)$ are the usual structure functions from deep-inelastic scattering processes at the factorization scale $\mu_{F}$ :

$$
\begin{aligned}
& F_{1}\left(x, \mu_{F}^{2}\right)=\sum_{q}\left(v_{q}^{2}+a_{q}^{2}\right)\left[q\left(x, \mu_{F}^{2}\right)+\bar{q}\left(x, \mu_{F}^{2}\right)\right] \\
& F_{2}\left(x, \mu_{F}^{2}\right)=2 x \sum_{q}\left(v_{q}^{2}+a_{q}^{2}\right)\left[q\left(x, \mu_{F}^{2}\right)+\bar{q}\left(x, \mu_{F}^{2}\right)\right]
\end{aligned}
$$




$$
F_{3}\left(x, \mu_{F}^{2}\right)=4 \sum_{q} v_{q} a_{q}\left[-q\left(x, \mu_{F}^{2}\right)+\bar{q}\left(x, \mu_{F}^{2}\right)\right]
$$

where $v_{q}\left(a_{q}\right)$ are the (axial) vector couplings of the quarks $q$ to the vector bosons $V: v_{q}=a_{q}=\sqrt{2}$ for $V=W$ and $v_{q}=2 I_{3 q}-4 e_{q} \sin ^{2} \theta_{W}, a_{q}=2 I_{3 q}$ for $V=Z$. $I_{3 q}$ is the third weak isospin component and $e_{q}$ the electric charge of the quark $q$.

In the past the NLO QCD corrections have been calculated first within the structure function approach [170. Since, at lowest order, the proton remnants are color singlets, no color will be exchanged between the first and the second incoming (outgoing) quark line and hence the QCD corrections only consist of the well-known corrections to the structure functions $F_{i}\left(x, \mu_{F}^{2}\right)(i=1,2,3)$. The final result for the NLO QCD-corrected cross section leads to the replacements

$$
\begin{aligned}
F_{i}\left(x, \mu_{F}^{2}\right) \rightarrow & F_{i}\left(x, \mu_{F}^{2}\right)+\Delta F_{i}\left(x, \mu_{F}^{2}, Q^{2}\right) \quad(i=1,2,3) \\
\Delta F_{1}\left(x, \mu_{F}^{2}, Q^{2}\right)= & \frac{\alpha_{s}\left(\mu_{R}\right)}{\pi} \sum_{q}\left(v_{q}^{2}+a_{q}^{2}\right) \int_{x}^{1} \frac{d y}{y}\left\{\frac{2}{3}\left[q\left(y, \mu_{F}^{2}\right)+\bar{q}\left(y, \mu_{F}^{2}\right)\right]\right. \\
& {\left[-\frac{3}{4} P_{q q}(z) \log \frac{\mu_{F}^{2} z}{Q^{2}}+\left(1+z^{2}\right) \mathcal{D}_{1}(z)-\frac{3}{2} \mathcal{D}_{0}(z)\right.} \\
& \left.+\frac{1}{4} g\left(y, \mu_{F}^{2}\right)\left[-2 P_{q g}(z) \log \frac{\mu_{F}^{2} z}{Q^{2}(1-z)}+4 z(1-z)-1\right]\right\} \\
\Delta F_{2}\left(x, \mu_{F}^{2}, Q^{2}\right)= & 2 x \frac{\alpha_{s}\left(\mu_{R}\right)}{\pi} \sum_{q}\left(v_{q}^{2}+a_{q}^{2}\right) \int_{x}^{1} \frac{d y}{y}\left\{\frac{2}{3}\left[q\left(y, \mu_{F}^{2}\right)+\bar{q}\left(y, \mu_{F}^{2}\right)\right]\right. \\
& {\left[-\frac{3}{4} P_{q q}(z) \log \frac{\mu_{F}^{2} z}{Q^{2}}+\left(1+z^{2}\right) \mathcal{D}_{1}(z)-\frac{3}{2} \mathcal{D}_{0}(z)\right.} \\
& \left.+\frac{1}{4} g\left(y, \mu_{F}^{2}\right)\left[-2 P_{q g}(z) \log \frac{\mu_{F}^{2} z}{Q^{2}(1-z)}+8 z(1-z)-1\right]\right\} \\
& \frac{\alpha_{s}\left(\mu_{R}\right)}{\pi} \sum_{q} 4 v_{q} a_{q} \int_{x}^{1} \frac{d y}{y}\left\{\frac{2}{3}\left[-q\left(y, \mu_{F}^{2}\right)+\bar{q}\left(y, \mu_{F}^{2}\right)\right]\right. \\
\Delta F_{3}\left(x, \mu_{F}^{2}, Q^{2}\right)= & {\left[-\frac{3}{4} P_{q q}(z) \log \frac{\mu_{F}^{2} z}{Q^{2}}+\left(1+z^{2}\right) \mathcal{D}_{1}(z)-\frac{3}{2} \mathcal{D}_{0}(z)\right.} \\
& \\
& \\
& \\
&
\end{aligned}
$$

where $z=x / y$ and the functions $P_{q q}, P_{q g}$ denote the Altarelli-Parisi splitting functions, which are given by 172

$$
\begin{aligned}
P_{q q}(z) & =\frac{4}{3}\left\{2 \mathcal{D}_{0}(z)-1-z+\frac{3}{2} \delta(1-z)\right\} \\
P_{q g}(z) & =\frac{1}{2}\left\{z^{2}+(1-z)^{2}\right\}
\end{aligned}
$$

The plus distributions are defined as

$$
\mathcal{D}_{i}(z)=\left(\frac{\log ^{i}(1-z)}{1-z}\right)_{+}
$$


The physical scale $Q$ is given by $Q^{2}=-q_{i}^{2}$ for $x=x_{i}(i=1,2)$. These expressions have to be inserted in Eq. (175) and the full result expanded up to NLO. The typical renormalization and factorization scales are fixed by the vector-boson momentum transfer $\mu_{R}=\mu_{F}=Q$. The size of the QCD corrections amounts to about 10\% and is thus small [170] as can be inferred from Fig. 25 that displays the individual corrections to the cross section [51]. These results have been extended by the calculation of the full NLO QCD corrections including interference contributions between the $s, t, u$-channels thus confirming the smallness of the additional contributions at NLO, too [171]. The NLO electroweak corrections reduce the cross section by about $10 \%$ [173] so that there is a significant interplay between the QCD and electroweak corrections, if the latter are defined in the $G_{F}$-scheme, see Fig. 25. For a reliable prediction of the vector-boson-fusion cross section both corrections have to be taken into account. The NLO QCD and electroweak corrections are also known exclusively and have been implemented in the public Monte Carlo programs HAWK [174] and VBFNLO [175. The full NLO results can also be generated with the MG5_AMC@NLO framework [167] and are available within the PowHEG box [165] so that a full matching to parton showers can be used. The NNLO QCD corrections have been obtained in the structure-function approach giving rise to a per-cent effect on the total cross section [176, 177], see Fig. 25. These are implemented in the public codes VBF@NnLO [176] and PROVBF [177] with the latter providing exclusive results. Up to NNLO non-factorizing contributions have been shown to be small [173, 176, 178]. The theoretical calculations have been extended to $\mathrm{N}^{3} \mathrm{LO}$ very recently [179]. The $\mathrm{N}^{3} \mathrm{LO}$ corrections are tiny, but reduce the theoretical uncertainties significantly.
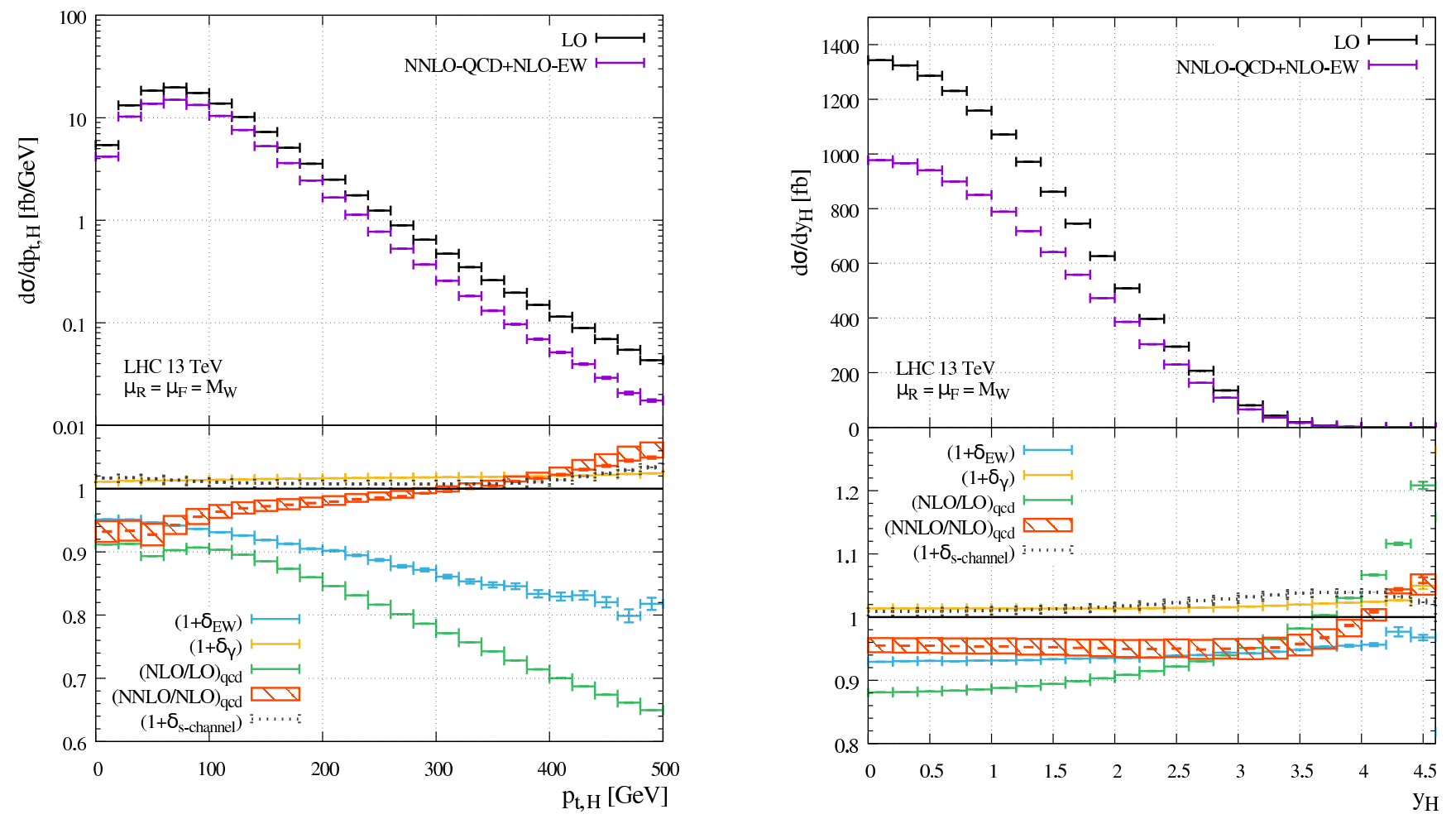

Figure 25: Transverse-momentum and rapidity distributions in VBF at LO and including the NNLO $Q C D$ and $N L O$ electroweak corrections for $\sqrt{s}=13 \mathrm{TeV}$ and $M_{H}=125 \mathrm{GeV}$. Upper plot: differential cross sections; lower plot: individual corrections. From Ref. [51] (page 90).

\subsubsection{Minimal supersymmetric extension}

Due to the absence of vector boson couplings to pseudoscalar Higgs particles $A$, only the scalar Higgs bosons $h, H$ can be produced via the vector-boson-fusion mechanism at tree level (see Fig. 26). However, 


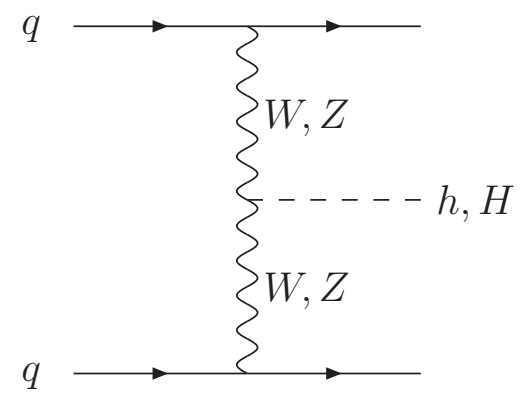

Figure 26: Diagram contributing to $q q \rightarrow q q V^{*} V^{*} \rightarrow q q h / q q H$ at lowest order.

these processes are suppressed with respect to the SM cross section due to the MSSM couplings $\left(g_{V}^{h / H}=\right.$ $\sin (\alpha-\beta) / \cos (\alpha-\beta))$,

$$
\sigma(p p \rightarrow q q \rightarrow q q+h / H)=\left(g_{V}^{h / H}\right)^{2} \sigma\left(p p \rightarrow q q \rightarrow q q H_{S M}\right)
$$

It turns out that the vector-boson-fusion mechanism is less relevant in the MSSM, because for large heavy scalar Higgs masses $M_{H}$, the MSSM couplings $g_{V}^{H}$ are very small apart from the decoupling regime where the light scalar Higgs boson becomes SM-like. The relative pure QCD corrections are the same as for the SM Higgs particle, i.e. 10\% [170]. The genuine SUSY-QCD corrections amount to less than a per cent and are thus small [180]. Some years ago the SUSY-electroweak corrections have been determined [181]. Being moderate in size in general they can modify the VBF cross section by $\sim 10 \%$.

\subsection{Higgs-strahlung: $q \bar{q} \rightarrow V^{*} \rightarrow V H$}

\subsubsection{Standard Model}

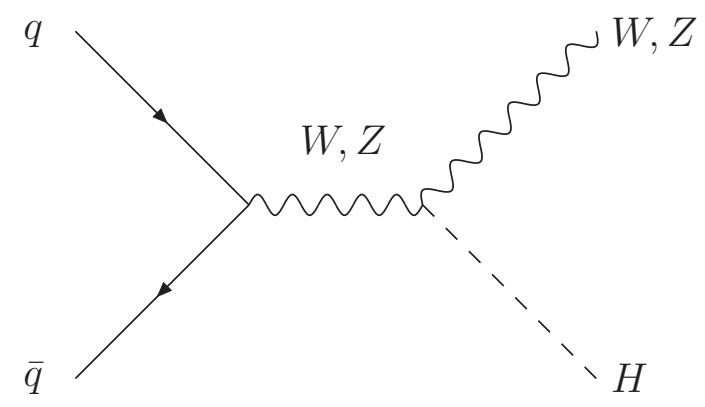

Figure 27: Diagram contributing to $q \bar{q} \rightarrow V^{*} \rightarrow V H$ at lowest order.

The Higgs-strahlung mechanism $q \bar{q} \rightarrow V^{*} \rightarrow V H(V=W, Z)$ (see Fig. 27) is important in the intermediate Higgs mass range due to the possibility to tag the associated vector boson and to reconstruct the $H \rightarrow b \bar{b}$ decay for strongly boosted Higgs bosons by using jet-substructure techniques [20, [182]. Its cross section is about one to two orders of magnitude smaller than the gluon-fusion cross section for the relevant Higgs mass range. The partonic cross section can be expressed at lowest-order as [18]

$$
\hat{\sigma}_{L O}(q \bar{q} \rightarrow V H)=\frac{G_{F}^{2} M_{V}^{4}}{288 \pi Q^{2}}\left(v_{q}^{2}+a_{q}^{2}\right) \sqrt{\lambda\left(M_{V}^{2}, M_{H}^{2} ; Q^{2}\right)} \frac{\lambda\left(M_{V}^{2}, M_{H}^{2} ; Q^{2}\right)+12 M_{V}^{2} / Q^{2}}{\left(1-M_{V}^{2} / Q^{2}\right)^{2}}
$$


where $\lambda(x, y ; z)=(1-x / z-y / z)^{2}-4 x y / z^{2}$ denotes the usual two-body phase-space factor and $v_{q}\left(a_{q}\right)$ are the (axial) vector couplings of the quarks $q$ to the vector bosons $V$, which have been defined after Eq. (176). At leading order the partonic c.m. energy squared $\hat{s}$ coincides with the invariant mass $Q^{2}=M_{V H}^{2}$ of the Higgs-vector-boson pair squared, $\hat{s}=Q^{2}$. The hadronic cross section can be obtained from convolving Eq. (183) with the corresponding $q \bar{q}$-luminosity,

$$
\sigma_{L O}(p p \rightarrow q \bar{q} \rightarrow V H)=\int_{\tau_{0}}^{1} d \tau \sum_{q} \frac{d \mathcal{L}^{q \bar{q}}}{d \tau} \hat{\sigma}_{L O}\left(Q^{2}=\tau s\right)
$$

with $\tau_{0}=\left(M_{H}+M_{V}\right)^{2} / s$ and $s$ the total hadronic c.m. energy squared and

$$
\frac{d \mathcal{L}^{q \bar{q}}}{d \tau}=\int_{\tau}^{1} \frac{d x}{x}\left[q\left(x, \mu_{F}^{2}\right) \bar{q}\left(\tau / x, \mu_{F}^{2}\right)+\bar{q}\left(x, \mu_{F}^{2}\right) q\left(\tau / x, \mu_{F}^{2}\right)\right]
$$

The NLO QCD corrections are identical to the corresponding corrections to the Drell-Yan process [183]. The natural scale of the process is given by the invariant mass of the Higgs-vector-boson pair in the final state, $\mu_{R}=\mu_{F}=Q$. The NLO QCD corrections increase the total cross section by about $30 \%$ and are thus moderate [183], see Fig. 28. Some time ago the NNLO QCD corrections have been determined [184. For the $Z H$ final state there is a sizeable contribution from the loop-induced $g g \rightarrow Z H$ process, see Fig. 29. It contributes $\sim 20 \%$ to the total cross section as can be inferred from Fig. 28, while the rest of the NNLO corrections amounts to about 5\%. Recently the NLO QCD corrections to $g g \rightarrow Z H$ have been calculated in the heavy-top-quark limit [185]. They increase this contribution significantly. The calculation of the QCD corrections has been supplemented by the full NLO electroweak corrections [186]. Within the $G_{F}$-scheme they decrease the cross section by about $10 \%$ and are thus of the same relevance as the NNLO QCD corrections, see Fig. 28. The NLO QCD and electroweak corrections are implemented in the public program HAWK [174] including leptonic decays of the final-state vector boson. The NNLO QCD corrections are implemented in the public codes HVNNLO [187] that includes final-state Higgs decays into leptons and bottom quarks, MCFM [188], VhNNLO [184], VH@NNLO [189] and NnLOPS [190]. The NLO results have been matched to parton showers within the Powheg box [165] and the MG5_AMC@NLO framework [167].

\subsubsection{Minimal supersymmetric extension}

For the same reasons as in the vector-boson-fusion mechanism case, the Higgs-strahlung off $W, Z$ bosons, $q \bar{q} \rightarrow V^{*} \rightarrow V h / V H(V=W, Z)$ (see Fig. 30), is smaller for the scalar MSSM Higgs particles $h, H$ than for the SM Higgs boson apart from the decoupling regime for the light scalar $h$. The cross sections can be easily related to the SM cross sections,

$$
\sigma(p p \rightarrow V+h / H)=\left(g_{V}^{h / H}\right)^{2} \sigma\left(p p \rightarrow V H_{S M}\right)
$$

which is true up to NNLO QCD apart from the loop-induced $g g \rightarrow Z+h / H$ process that involves the large top Yukawa coupling in addition to the $H Z Z$ coupling, see Fig. 29. Pseudoscalar couplings to intermediate vector bosons are absent so that pseudoscalar Higgs particles cannot be produced at tree level in this channel. The relative QCD corrections are the same as in the SM case and thus of moderate size 183 in general up to NNLO. Only the contamination from $g g \rightarrow Z+h / H$ will be weighted differently due to the top Yukawa coupling factor. Its contribution ranges at about 20\%. The genuine SUSY-QCD corrections are small [180], while the genuine SUSY-electroweak corrections are unknown so far. 

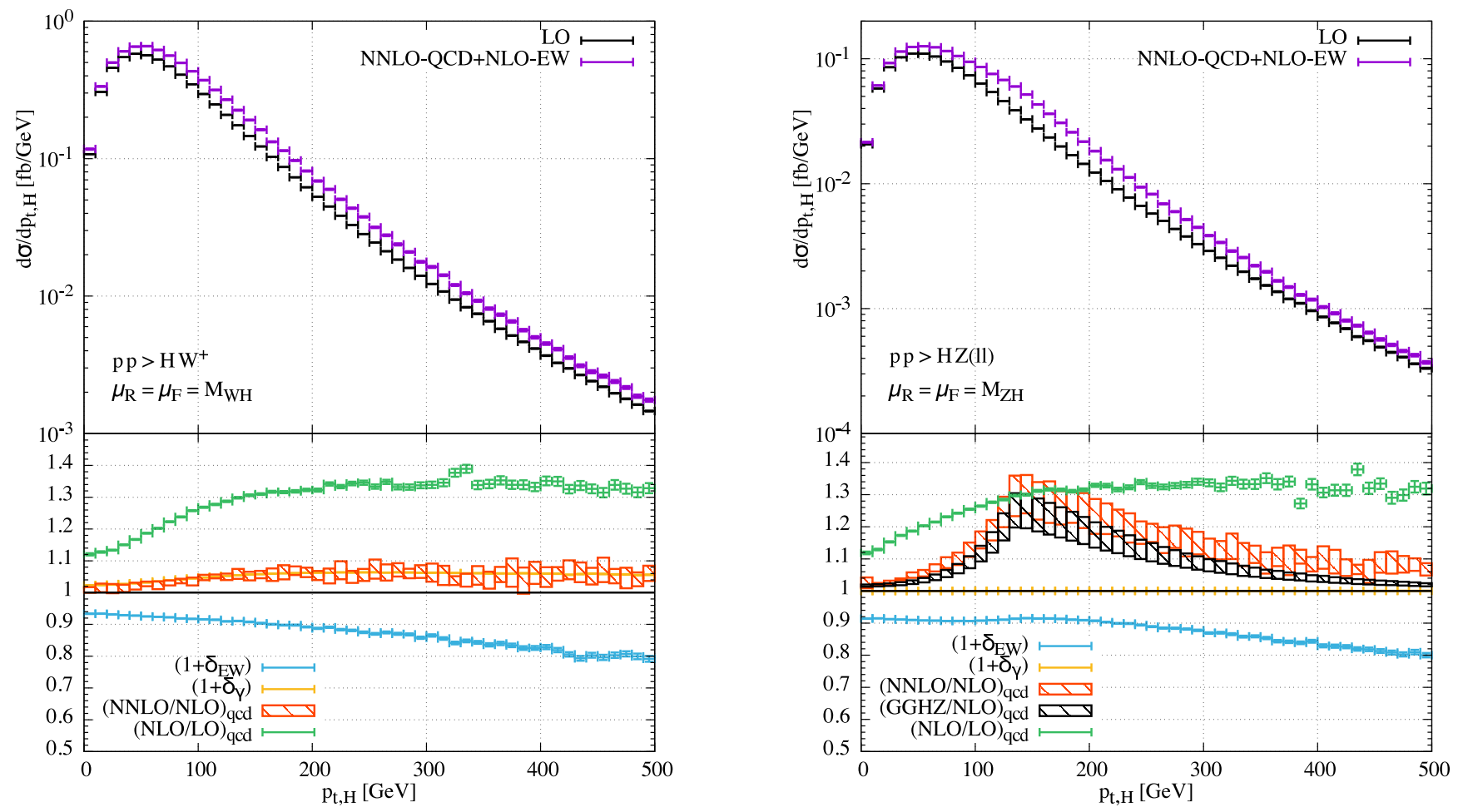

Figure 28: Transverse-momentum distributions of $W^{+} H$ (left) and $Z H$ (right) production at $L O$ and including the NNLO QCD and NLO electroweak corrections for $\sqrt{s}=13$ TeV and $M_{H}=125$ GeV. Upper plot: differential cross sections; lower plot: individual corrections. From Ref. [51] (pages 100, 104).

\subsection{Higgs bremsstrahlung off top and bottom quarks}

\subsubsection{Standard Model}

$t \bar{t} H$ production. In the intermediate Higgs mass range the cross section of the associated production of the Higgs boson with a $t \bar{t}$ pair is smaller than those of the Higgs-strahlung processes [19], but of significant size, since it allows to tag the additional $t \bar{t}$ pair and the Higgs boson decays $H \rightarrow b \bar{b}$, $H \rightarrow W W$ as well as the rare photonic decay mode $H \rightarrow \gamma \gamma$ on the long time run. This process is generated by gluon-gluon and $q \bar{q}$ initial states at leading order (see Fig. 31). At the LHC the gluon-gluon channel is dominant due to the enhanced gluon structure function analogous to the gluonfusion mechanism. The NLO QCD corrections to $t \bar{t} H$ production have been calculated and result in a moderate increase of the cross section by $\sim 20 \%$ [191, 192, 193. The origin of this moderate size is the strong phase-space suppression of the massive three-particle threshold so that soft and collinear
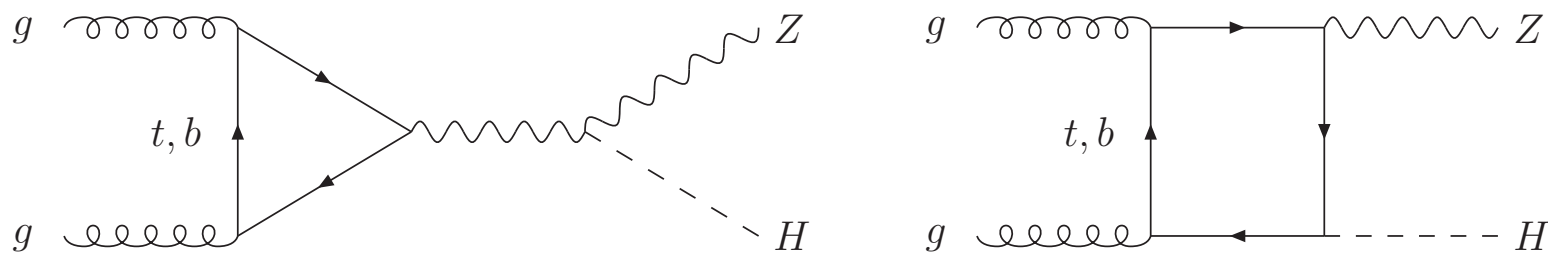

Figure 29: Diagrams contributing to $g g \rightarrow Z H$ at $N N L O$. 


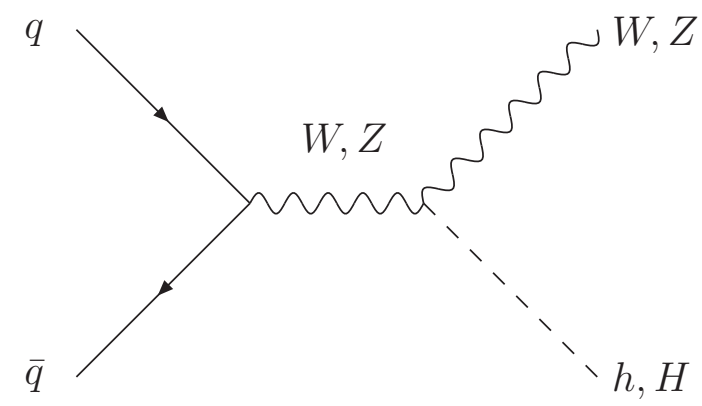

Figure 30: Diagram contributing to $q \bar{q} \rightarrow V^{*} \rightarrow h / H+V$ at lowest order.
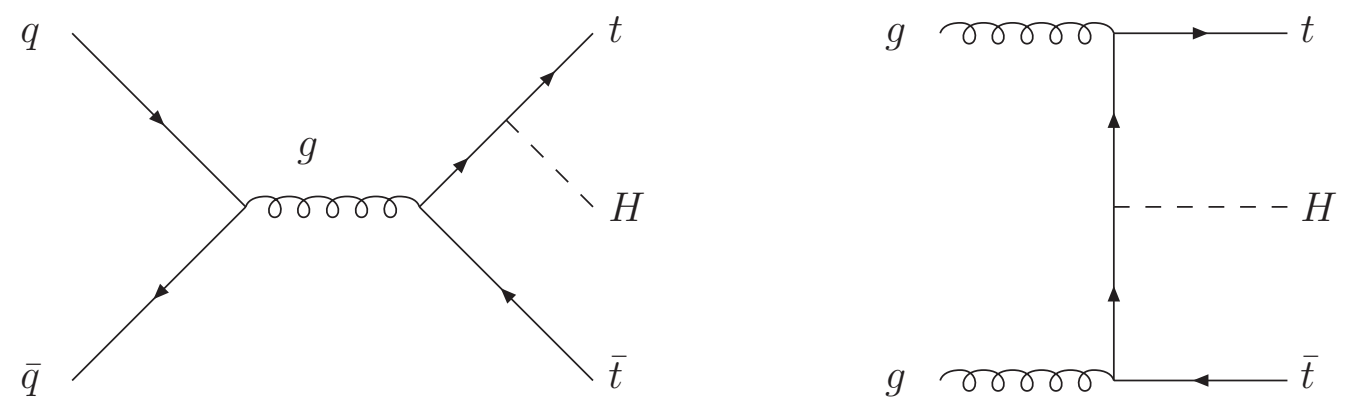

Figure 31: Typical diagrams contributing to $q \bar{q} / g g \rightarrow t \bar{t} H$ at lowest order.

threshold effects are strongly diminished. The main parts of the QCD corrections originate from regions significantly above the production threshold and can be approximated by a fragmentation approach involving first producing a $t \bar{t}$ pair supplemented by the $t \rightarrow t H$ fragmentation in the high-energy limit [191, 194]. Although this provides a bad approximation for the magnitude of the cross section itself it leads to a reasonable estimate of the relative QCD corrections [191. The full NLO results have recently been implemented in the Powheg box [195], matched to Sherpa [196] and generated within the MG5_AMC@NLO framework [193] thus offering NLO event generators matched to parton showers. The NLO result has recently been improved by a soft and collinear gluon resummation based on the SCET approach starting from the boosted final-state particle triplet11] [198] leading to a further increase of the cross section by $5-10 \%$. The residual scale dependence is reduced to the level of $5-10 \%$. Recently the electroweak corrections have been calculated for $t \bar{t} H$ production [199]. They range a the per-cent level and are thus small. Moreover, off-shell top-quark effects have been determined at NLO in QCD [200] with leptonic top-quark decays and turn out to be small for the inclusive $t \bar{t} H$ cross section. However, they play a role in certain regions of phase space and are thus of relevance for distributions.

$b \bar{b} H$ production. Higgs bremsstrahlung off bottom quarks does not play a significant role for the SM Higgs boson, but yields an important constraint on the bottom Yukawa coupling. Its total cross section is of similar size as the $t \bar{t} H$ production cross section. The results of $t \bar{t} H$ production can be taken over for $b \bar{b} H$ production. However, they have to be transformed to the four-flavour-scheme (4FS) in order to avoid artificial large logarithms initiated by the bottom mass in the combination of the virtual and real corrections at NLO. In this way finite bottom-mass effects can be taken into account consistently. The NLO QCD corrections are positive and large. There is a decrease by about $10 \%$ due

\footnotetext{
${ }^{11}$ The recent alternative approach using conventional threshold resummation techniques does not yield a sizeable contribution beyond NLO [197] due to the strong threshold suppression.
} 
to top-Yukawa induced contributions at NLO corresponding to the diagrams of Fig. 32 [201]. However,
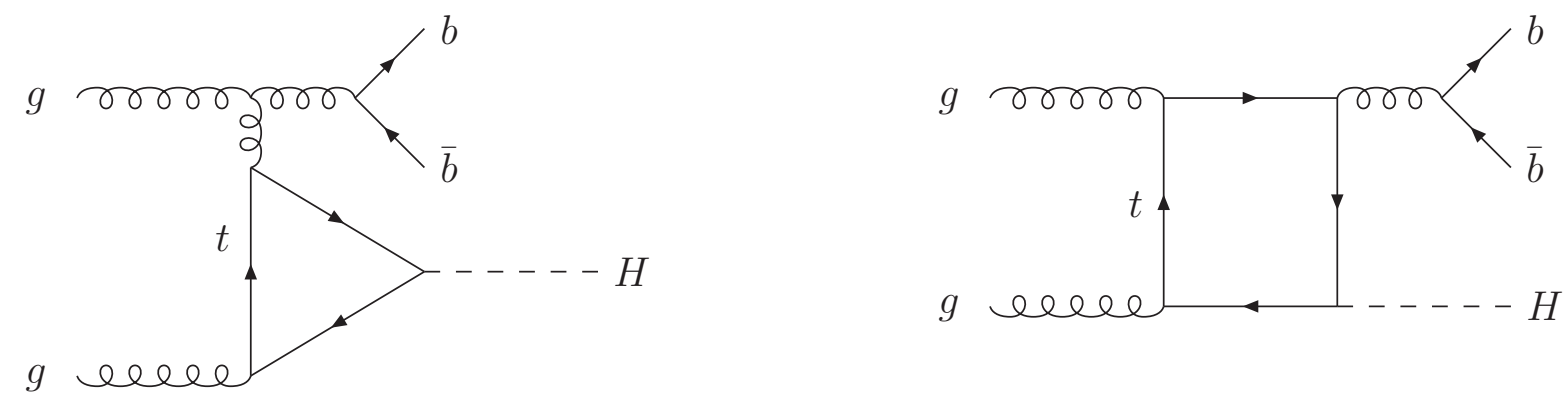

Figure 32: Typical diagrams contributing to $g g \rightarrow b \bar{b} H$ at NLO involving the top Yukawa coupling.

the $p_{T}$-integration of the final-state bottom quarks generates potentially large logarithmic contributions as part of the $4 \mathrm{FS}$ result,

$$
\hat{\sigma}(g g \rightarrow b \bar{b} H)=\int_{\tau_{H}}^{1} d x_{1} \int_{\tau_{H} / x_{1}}^{1} d x_{2} \hat{b}\left(x_{1}, Q^{2}\right) \hat{b}\left(x_{2}, Q^{2}\right) \hat{\sigma}(b \bar{b} \rightarrow H)+\Delta \sigma
$$

where the effective bottom densities are given by

$$
\hat{b}\left(x, Q^{2}\right)=P_{q g}(x) \log \frac{Q^{2}}{M_{b}^{2}}
$$

with a scale $Q$ significantly smaller than the Higgs boson mass as can be inferred from the $p_{T}$ distribution of the bottom quark in the final state [202]. The logarithmic contributions contained in $\hat{b}\left(x, Q^{2}\right)$ can be resummed by the DLGLAP evolution [172 of the initial-state bottom PDFs. This requires to treat the bottom quark as a massless active flavour, i.e. to introduce the 5FS with bottom densities of the proton.

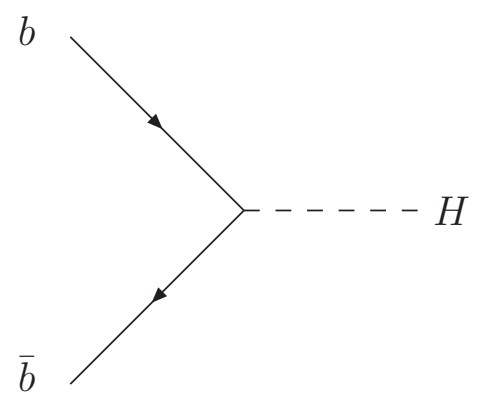

Figure 33: Diagram contributing to $b \bar{b} \rightarrow H$ within the $5 F S$ at lowest order.

The 5FS-calculation starts from the process $b \bar{b} \rightarrow H$ at LO, see Fig. 33, yielding the LO cross section

$$
\begin{aligned}
\sigma_{L O}(p p \rightarrow H+X) & =\sigma_{0} \tau_{H} \frac{d \mathcal{L}^{b \bar{b}}}{d \tau_{H}} \\
\sigma_{0} & =\frac{\pi G_{F} \bar{m}_{b}^{2}\left(\mu_{R}\right)}{N_{c} \sqrt{2} M_{H}^{2}}
\end{aligned}
$$

with the variable $\tau_{H}=M_{H}^{2} / s$ involving the hadronic c.m. energy squared $s$ and the parton luminosity

$$
\frac{d \mathcal{L}^{b \bar{b}}}{d \tau}=\int_{\tau}^{1} \frac{d x}{x}\left[b\left(x, \mu_{F}^{2}\right) \bar{b}\left(\tau / x, \mu_{F}^{2}\right)+\bar{b}\left(x, \mu_{F}^{2}\right) b\left(\tau / x, \mu_{F}^{2}\right)\right]
$$




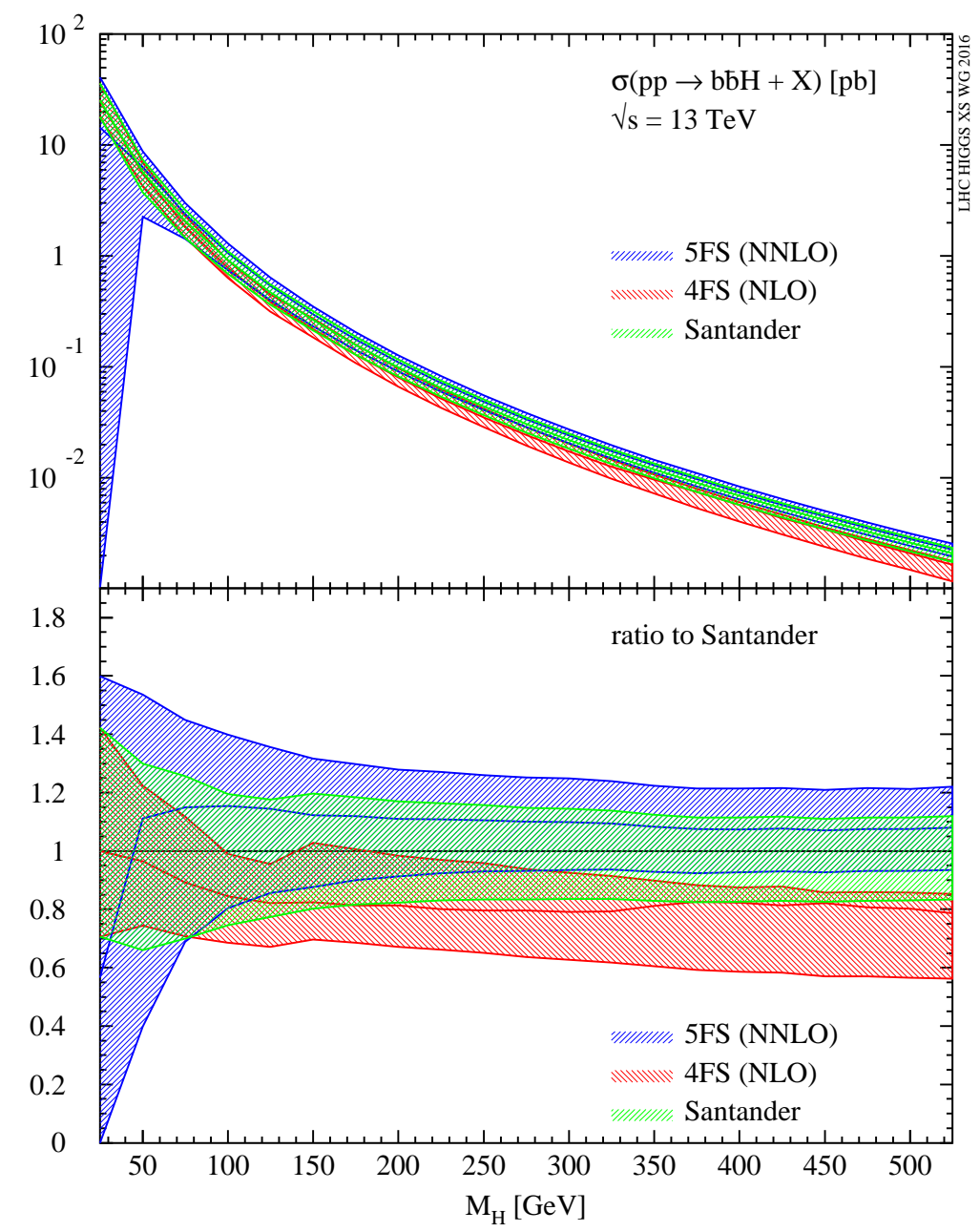

Figure 34: Comparison of the inclusive $b \bar{b} H$ production cross sections for the $4 F S$ and $5 F S$ as well as the result using Santander matching. The lower plot displays the ratios to the central Santander-matched prediction. The bands reflect the combined uncertainties. From Ref. [51] (page 523).

The 5FS relies on three approximations at LO, i.e. neglecting bottom-mass and off-shell effects and the transverse momenta of the initial-state bottom quarks. The last two conditions are resolved by adding the higher-order QCD corrections so that only the inclusion of bottom mass effects is not possible in the 5FS framework. In the small-mass limit the 4FS- and 5FS-calculations have to approach each other at higher orders so that the comparison of both schemes yields an estimate of missing higher-order corrections. Using the running $\overline{\mathrm{MS}}$ bottom mass $\bar{m}_{b}^{2}\left(\mu_{R}\right)$ the NLO QCD corrections to $b \bar{b} \rightarrow H$ have been calculated [203],

$$
\begin{aligned}
\sigma(b \bar{b} \rightarrow H) & =\sigma_{L O}+\Delta \sigma_{b \bar{b}}+\Delta \sigma_{b g} \\
\Delta \sigma_{b \bar{b}} & =\frac{\alpha_{s}\left(\mu_{R}\right)}{\pi} \int_{\tau_{H}}^{1} d \tau \sum_{q} \frac{d \mathcal{L}^{b \bar{b}}}{d \tau} \sigma_{0} z \omega_{b \bar{b}}(z) \\
\Delta \sigma_{b g} & =\frac{\alpha_{s}\left(\mu_{R}\right)}{\pi} \int_{\tau_{H}}^{1} d \tau \sum_{b, \bar{b}} \frac{d \mathcal{L}^{b g}}{d \tau} \sigma_{0} z \omega_{b g}(z)
\end{aligned}
$$

with $z=\tau_{H} / \tau$, the coefficient functions

$\omega_{b \bar{b}}(z)=-P_{q q}(z) \log \frac{\mu_{F}^{2}}{\tau s}+\frac{4}{3}\left\{\left(2 \zeta_{2}-1+\frac{3}{2} \log \frac{\mu_{R}^{2}}{M_{H}^{2}}\right) \delta(1-z)+\left(1+z^{2}\right)\left[2 \mathcal{D}_{1}(z)-\frac{\log z}{1-z}\right]+1-z\right\}$ 
$\omega_{b g}(z)=-\frac{1}{2} P_{q g}(z) \log \left(\frac{\mu_{F}^{2}}{(1-z)^{2} \tau s}\right)-\frac{1}{8}(1-z)(3-7 z)$

and the parton luminosity

$$
\frac{d \mathcal{L}^{b g}}{d \tau}=\sum_{b, \bar{b}} \int_{\tau}^{1} \frac{d x}{x}\left[b\left(x, \mu_{F}^{2}\right) g\left(\tau / x, \mu_{F}^{2}\right)+g\left(x, \mu_{F}^{2}\right) b\left(\tau / x, \mu_{F}^{2}\right)\right]
$$

The calculation of the QCD corrections to $b \bar{b} \rightarrow H$ has been extended to NNLO [204]. The QCD corrections decrease the cross section by about $30 \%$ for the central scale choice equal to the Higgs mass. At NNLO the $g g$-initiated diagrams of the 4FS (see Fig. 31) contribute for the first time so that the kinematics of the spectator bottom quarks is restored. For a better comparison of both schemes a general analysis of the logarithms related to the $p_{T}$-integrated bottom quarks has been performed with the result that the proper factorization scale to be chosen for the bottom densities is significantly smaller than the Higgs boson mass within the 5FS 202. This analysis has been extended to all orders for the logarithmic terms and confirms the smaller effective factorization scale in the 5FS [205]. This decreases the 5FS result and makes it agree better with the NLO 4FS result. In order to combine both calculations within the 4FS and 5FS one has to avoid double counting of common contributions according to Eq. (187). This has been done empirically in the past by using the Santander matching [206]

$$
\begin{aligned}
\sigma & =\frac{\sigma^{4 F S}+w \sigma^{5 F S}}{1+w} \\
w & =\log \frac{M_{H}}{m_{b}}-2
\end{aligned}
$$

where the chosen weight $w$ pays attention to the fact that the common terms of both calculations are logarithmic. Fig. 34 shows the comparison of the NLO 4FS result with the NNLO 5FS and Santandermatched cross section as a function of the Higgs mass.

This empirically matched result has been improved by two different procedures of a consistent matching of the 4FS and 5FS, one dubbed 'FONLL' 207] and the other 'NLO+NNLL ${ }_{\text {partial }}+$ ybyt [208]. Both approaches reexpress the quantities of one scheme in terms of the other one and use suitably modified sets of the PDF4LHC15 parton density functions that treat the particular region at the input scale of the size of the bottom mass consistent with the matching procedures [51]. The perturbative counting within both approaches is different so that a comparison of both approaches is motivated to obtain an idea of their differences. Both results are shown together with the Santander-matched result in Fig. 35 as a function of the Higgs mass. Both consistent matching procedures agree well within their respective uncertainties and come out slightly higher than the cross sections obtained by Santander matching by about 20\%. Both consistently matched results indicate a tendency towards the original 5FS calculation for larger Higgs boson masses.

$t H$ production. Single-top quark production in association with a Higgs boson proceeds in two different generic ways at LO, the dominant $t$-channel contribution and the subleading $s$-channel one, see Fig. 36. It allows to test the sign of the top Yukawa coupling, since there are interference effects between the Higgs-boson couplings to top quarks and $W$ bosons. The $t$-channel suffers from the same problem as $b \bar{b} H$ production, i.e. there is the alternative to treat it within the 4FS or 5FS the latter starting with bottom densities of the proton the DGLAP evolution of which resum potentially large logarithms arising in the $4 \mathrm{FS}$. Within the $5 \mathrm{FS}$ there is no interference between the $t$ - and $s$-channel contributions up to NLO. The full NLO calculation has been performed by means of the MG5_AMC@NLO framework [209]. The QCD corrections to the $t$-channel contribution are small, i.e. $\sim 10 \%$, and of a 


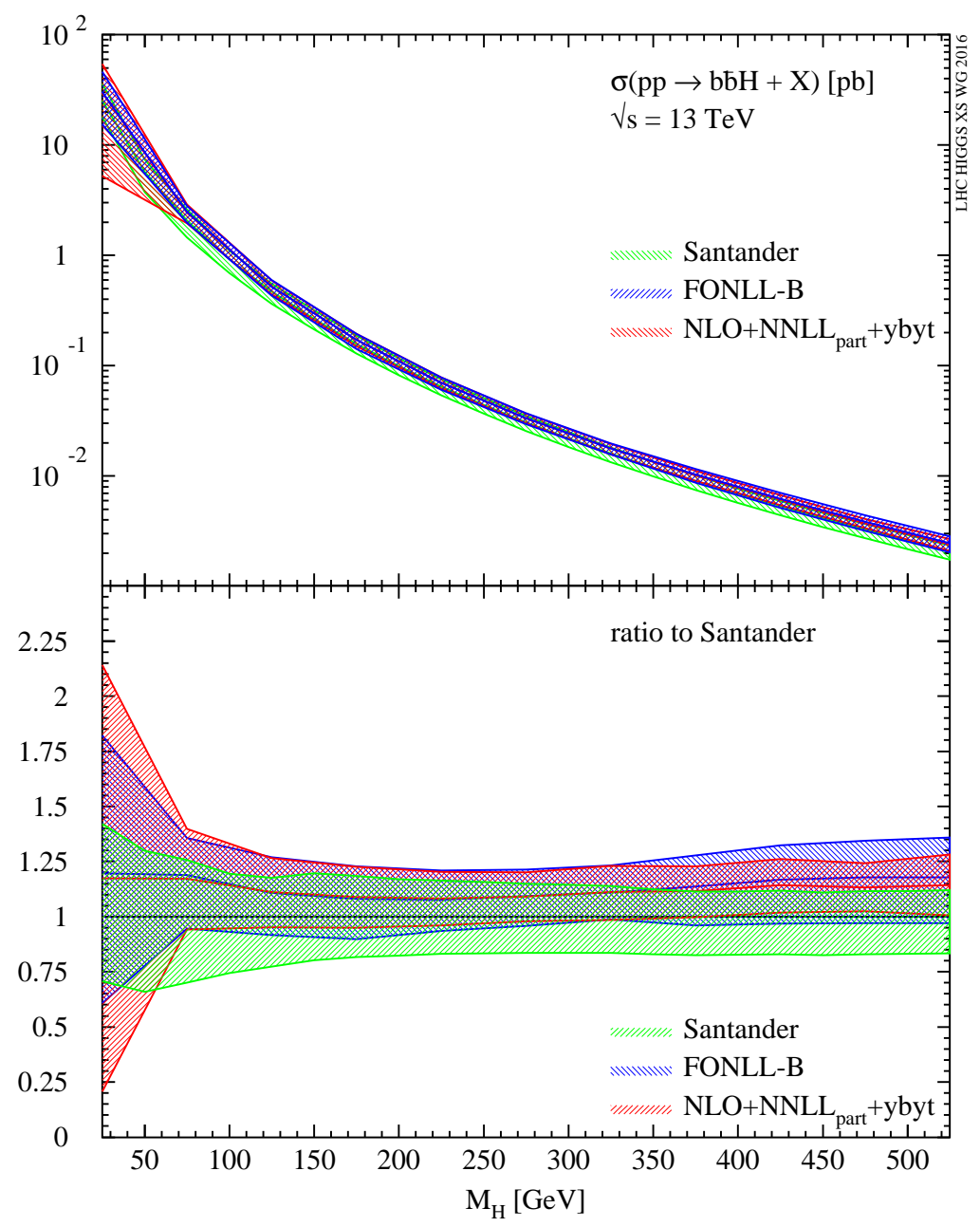

Figure 35: Comparison of the inclusive $b \bar{b} H$ production cross sections for the different matching procedures, i.e. Santander matching, FONLL-B [207] and $N L O+N N L L_{\text {partial }}+y b y t$ [208]. The lower plot displays the ratios to the central Santander-matched prediction. The bands reflect the combined uncertainties. From Ref. [51] (page 524).

similar size as the difference between the 4FS and 5FS at NLO. In order to reduce the difference between the 4FS and 5FS the central scale choice has been reduced to $\mu_{R}=\mu_{F}=\left(M_{H}+M_{t}\right) / 4$ for the $t$-channel contributions, while the $s$-channel uses a central scale twice as large [51]. The theoretical uncertainties, estimated from the scale dependence and the difference between both schemes, range at the level of 5-15\% [51]. Including the $\mathrm{PDF}+\alpha_{s}$ uncertainties the total uncertainty can be estimated to be in the same ball park.

\subsubsection{Minimal supersymmetric extension}

$t \bar{t} / b \bar{b}+\Phi$ production. The scalar Higgs cross sections for Higgs bremsstrahlung off heavy quarks $Q$ can simply be related to the SM case at LO:

$$
\sigma(p p \rightarrow h / H+Q \bar{Q})=\left(g_{Q}^{h / H}\right)^{2} \sigma\left(p p \rightarrow H_{S M} Q \bar{Q}\right)
$$

This relation is also valid at NLO apart from diagrams involving closed top/bottom loops coupling to the Higgs bosons, see Fig. 32. The LO expressions for the pseudoscalar Higgs boson [169] are similarly involved as the scalar case and will not be presented here.

The top quark coupling to MSSM Higgs bosons is suppressed with respect to the $\mathrm{SM}$ for $\operatorname{tg} \beta>1$. Therefore Higgs bremsstrahlung off top quarks $p p \rightarrow \Phi t \bar{t}$ is less important for MSSM Higgs particles. 
(a)

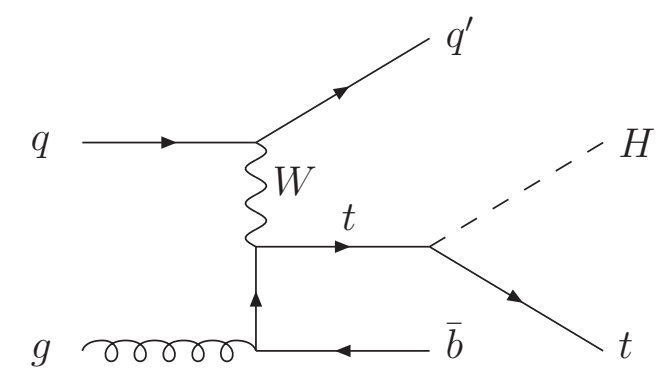

(b)

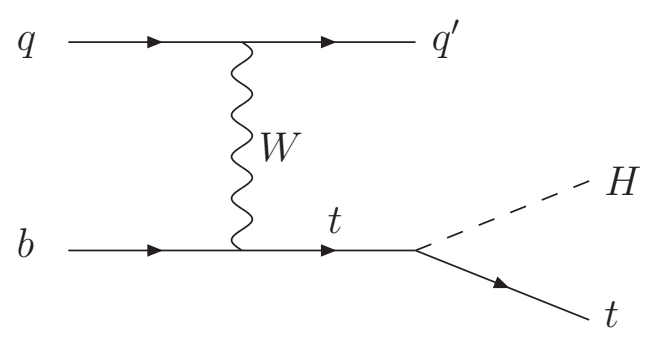

(c)

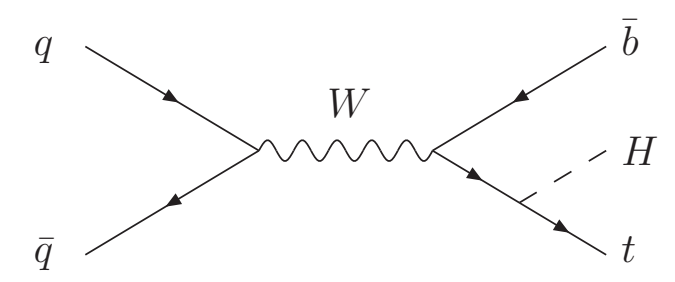

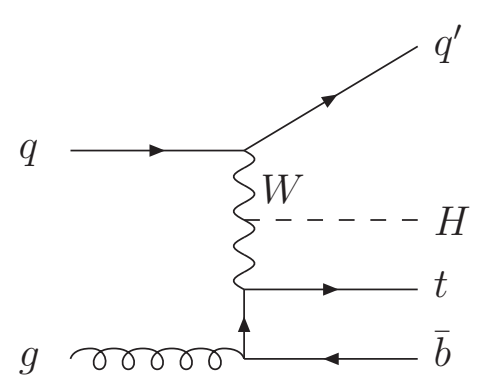
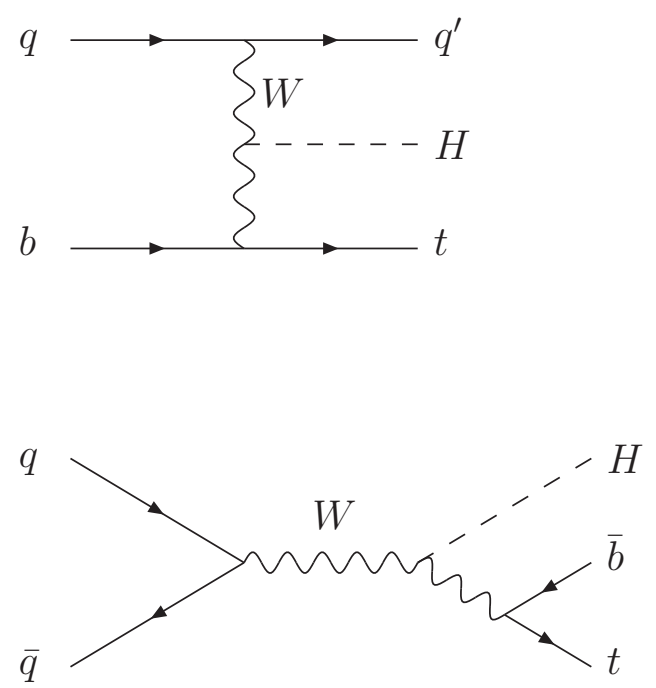

Figure 36: Typical diagrams contributing to $t H$ production at lowest order: (a) t-channel within the $4 F S$, (b) t-channel within the 5FS, (c) s-channel.

On the other hand Higgs bremsstrahlung off bottom quarks $p p \rightarrow H b \bar{b}$ will be the dominant Higgs production channel for large $\operatorname{tg} \beta$ due to the strongly enhanced bottom quark Yukawa couplings [19]. The QCD corrections for the scalar Higgs bosons can be inferred from the SM cross section by Eq. (195) but with the properly rescaled diagrams of Fig. 32. The NLO QCD corrections to pseudoscalar $t \bar{t} A$ production have been calculated in Ref. [193] yielding a slightly larger but moderate increase of the cross section. The full SUSY-QCD corrections have been calculated quite recently for $t \bar{t} \phi$ production [210, 211] and for $b \bar{b} \phi$ production in the 4FS [210] and the 5FS [212]. Being of moderate size for small values of $\operatorname{tg} \beta$, i.e. similar to the pure QCD corrections, they can be large for $b \bar{b} \phi$ production due to the $\Delta_{b}$ corrections to the bottom Yukawa couplings. However, the remainder after absorbing the leading terms in the effective resummed Yukawa couplings turns out to be small [210]. The SUSY-electroweak corrections are known for $b \bar{\phi}$ production within the 5FS [213. The latter can be well approximated by the radiatively improved Higgs masses and couplings and the electroweak $\Delta_{b}$ terms for large values of $\operatorname{tg} \beta$.

Charged Higgs production. Charged Higgs bosons are dominantly produced at the LHC in association with top quarks $g g, q \bar{q} \rightarrow t \bar{b} H^{-}$and the charge-conjugated process, see Fig. 38A. For charged Higgs-boson masses $M_{H^{ \pm}}<m_{t}-m_{b}$ the dominant contribution to this process factorizes into $t \bar{t}$ pair production with the subsequent (anti)top decay into a charged Higgs and (anti)bottom quark. This region is then suitably described by the QCD- and electroweak-corrected $t \bar{t}$ production cross section [214, 215] multiplied with the radiatively corrected branching ratio of the top decays [216, 217]. However, for larger charged Higgs masses the top decays are kinematically closed and charged Higgs bosons 

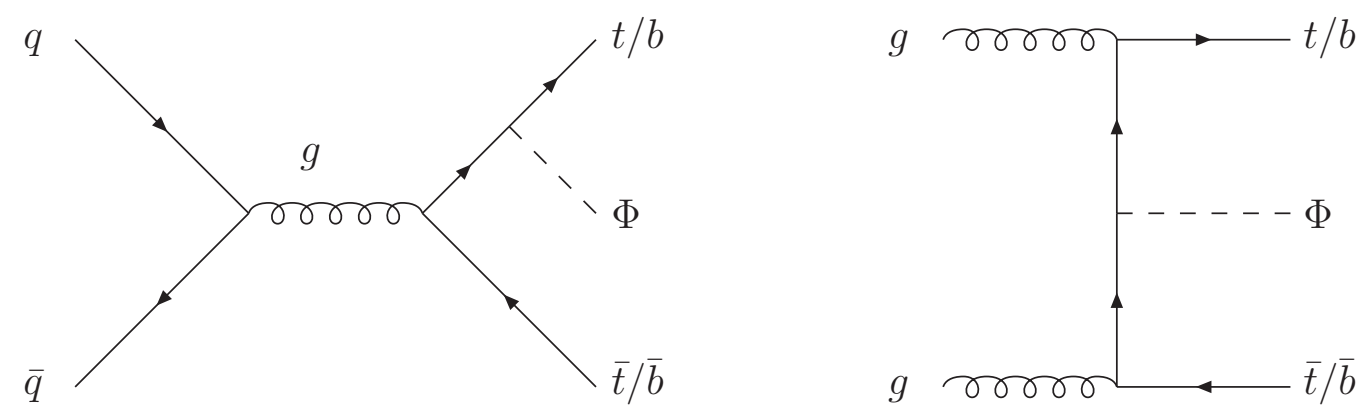

Figure 37: Typical diagrams contributing to $q \bar{q} / g g \rightarrow \Phi Q \bar{Q} \quad(Q=t, b)$ at lowest order.

are produced in terms of the full process of Fig. 383. For the inclusive rate the final-state bottom quark gives rise to the options to calculate this process in the $4 \mathrm{FS}$ or the $5 \mathrm{FS}$, where the latter starts from the process $g b \rightarrow t H^{-}$and the charge-conjugated process and thus uses bottom PDFs of the proton. Both calculations have been performed up to NLO QCD resulting in moderate scale dependences [218, 219]. While the 5FS is available exclusively for all distributions involving the top quark and charged Higgs boson, the 4FS allows to determine distributions involving the bottom quark in the final state in addition. Both calculations have been combined by means of the Santander matching of Eq. (194) [206] with the weight $w=\log M_{H^{ \pm}} / m_{b}-2$. For small values of $\operatorname{tg} \beta$ the genuine SUSY-QCD corrections are of moderate size [218, 219], while for $\operatorname{large} \operatorname{tg} \beta$ they are completely dominated by $\Delta_{b}$-terms with a small remainder. Thus for the compilation of the charged-Higgs production cross section the effective bottom Yukawa coupling

$$
\tilde{g}_{b}^{H^{ \pm}}=\tilde{g}_{b}^{A}
$$

(combined with the top Yukawa coupling factor $g_{t}^{H^{ \pm}}=g_{t}^{A}$ ) has been used that can be translated into an effective $\operatorname{tg} \beta$ value [220]. The result for the continuum production cross section without SUSY-QCD corrections is shown in Fig. 39 for two charged Higgs masses as a function of $\operatorname{tg} \beta$. The QCD corrections enhance the 4FS cross section by about $60 \%$ [218], while they are moderate for the 5FS [219].

An open problem for a long time has been the treatment of the intermediate region between resonant top quark decays and the continuum contribution for large charged Higgs masses. This has been solved recently by a full NLO calculation in the 4FS within the complex-mass scheme for the intermediate top quarks [221]. The NLO QCD corrections turn out to be large in this mass regime, too, while the results nicely interpolate between the low- and high-mass regimes as can be inferred from Fig. 40.

The second important charged Higgs production process is charged Higgs pair production in a Drell-Yan type process (see Fig. 38b) [222]

$$
p p \rightarrow q \bar{q} \rightarrow H^{+} H^{-}
$$

which is mediated by $s$-channel photon and Z-boson exchange. The NLO QCD corrections can be taken from the Drell-Yan process and are of moderate size as in the case of the neutral Higgs-strahlung process discussed before [180]. The genuine SUSY-QCD corrections, mediated by virtual gluino and squark exchange in the initial state, are small [180].

Charged Higgs pairs can also be produced from $g g$ initial states by the loop-mediated process [223, 224] (see Fig. 38k)

$$
p p \rightarrow g g \rightarrow H^{+} H^{-}
$$

where the dominant contributions emerge from top and bottom quark loops as well as stop and sbottom loops, if the squark masses are light enough. The NLO corrections to this process are unknown. This cross section is of similar size as the bottom-initiated process [224] (see Fig. 38])

$$
p p \rightarrow b \bar{b} \rightarrow H^{+} H^{-}
$$




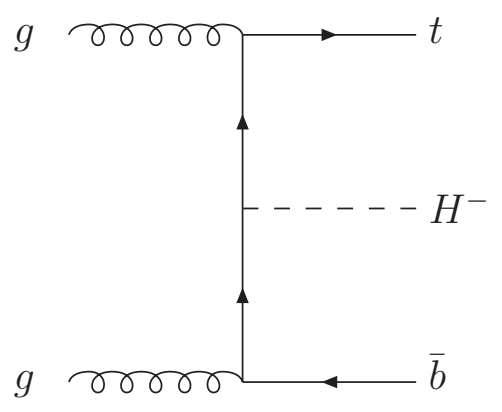

(a)

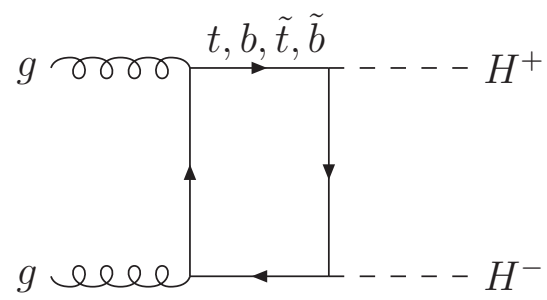

(c)

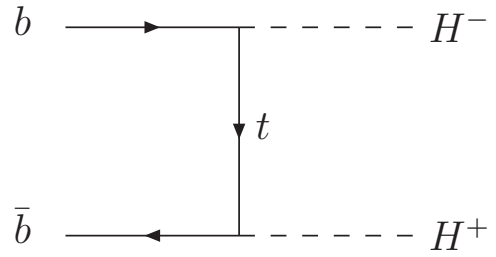

(e)

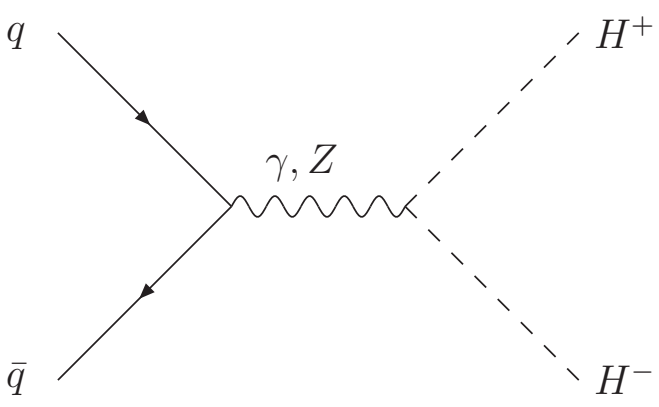

(b)

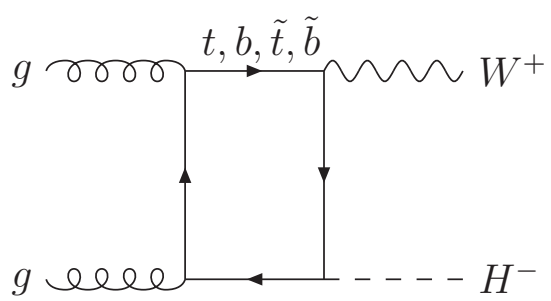

(d)

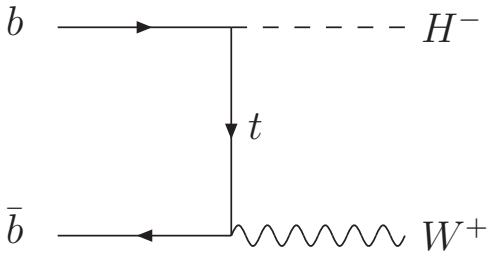

$(f)$

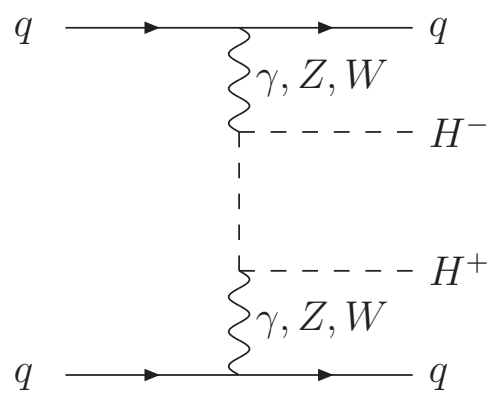

(g)

Figure 38: Typical diagrams for charged Higgs boson production mechanisms at leading order: (a) $g g \rightarrow H^{-} t \bar{b}$, (b) $q \bar{q} \rightarrow H^{+} H^{-}$, (c) $g g \rightarrow H^{+} H^{-}$, (d) $g g \rightarrow W^{+} H^{-}$, (e) $b \bar{b} \rightarrow H^{+} H^{-}$, (f) $b \bar{b} \rightarrow W^{+} H^{-}$, (g) $q q \rightarrow q q H^{+} H^{-}$. 

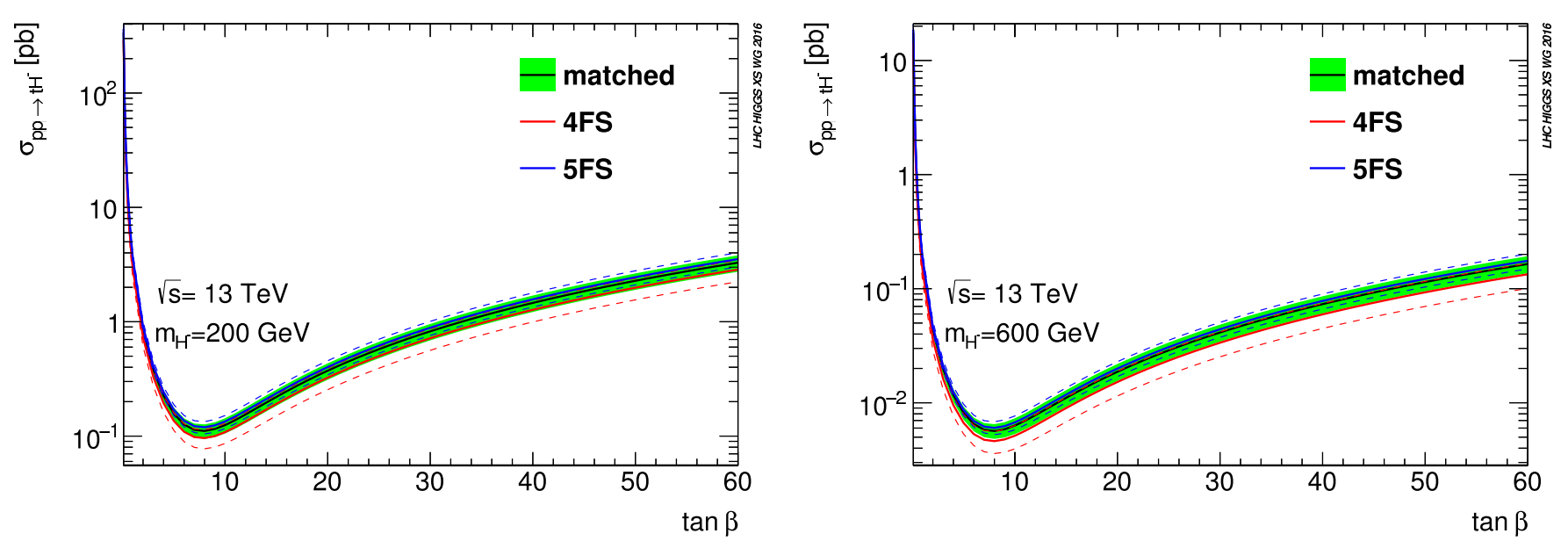

Figure 39: Charged Higgs production cross section in association with top quarks as functions of tg $\beta$ for two values of the charged Higgs mass. The dashed lines show the uncertainty bands of the $4 F S$ and $5 F S$, while the green bands are the combined results with Santander-matching. From Ref. [51] (page 543).

which relies on the approximations required by the introduction of the bottom densities as discussed before and is known at NLO [225]. The SUSY-QCD corrections are of significant size due to the $\Delta_{b}$ terms related to the bottom Yukawa coupling. The pure QCD corrections and the genuine SUSY-QCD corrections can be of opposite sign.

Charged Higgs bosons can be produced in association with a $W$ boson [226, 227] (see Fig. 38d)

$$
p p \rightarrow g g \rightarrow H^{+} W^{-} \quad \text { and c.c. }
$$

which is generated by top-bottom quark loops and stop-sbottom loops, if the squark masses are small enough. This process is known at LO only. The same final state also arises from the process [226, 228] (see Fig. 38f)

$$
p p \rightarrow b \bar{b} \rightarrow H^{+} W^{-} \quad \text { and c.c. }
$$

which is based on the approximations of the 5FS. The QCD corrections have been calculated and turn out to be of moderate size [229].

Finally, charged Higgs-boson pairs can be produced in vector-boson-fusion [230] (see Fig. 38g)

$$
q q \rightarrow q q V^{*} V^{*} \rightarrow q q H^{+} H^{-}
$$

The LO cross section is independent of $\operatorname{tg} \beta$ and can be sizeable within the MSSM. However, the calculation of Ref. [230] is not consistent with the parton picture, since small quark masses have been introduced for the accompanying quarks in order to regulate the collinear divergences of photon-exchange contributions. For a more reliable prediction QED-mass factorization has to be performed already at LO.

\subsection{Summary of single-Higgs boson production cross sections}

\subsubsection{Standard Model}

All Higgs boson production cross sections have been updated with the known higher-order corrections and the most recent parton density functions, i.e. the PDF4LHC15 sets [141], where NLO densities have been used consistently for NLO predictions and NNLO densities for NNLO predictions. Using 


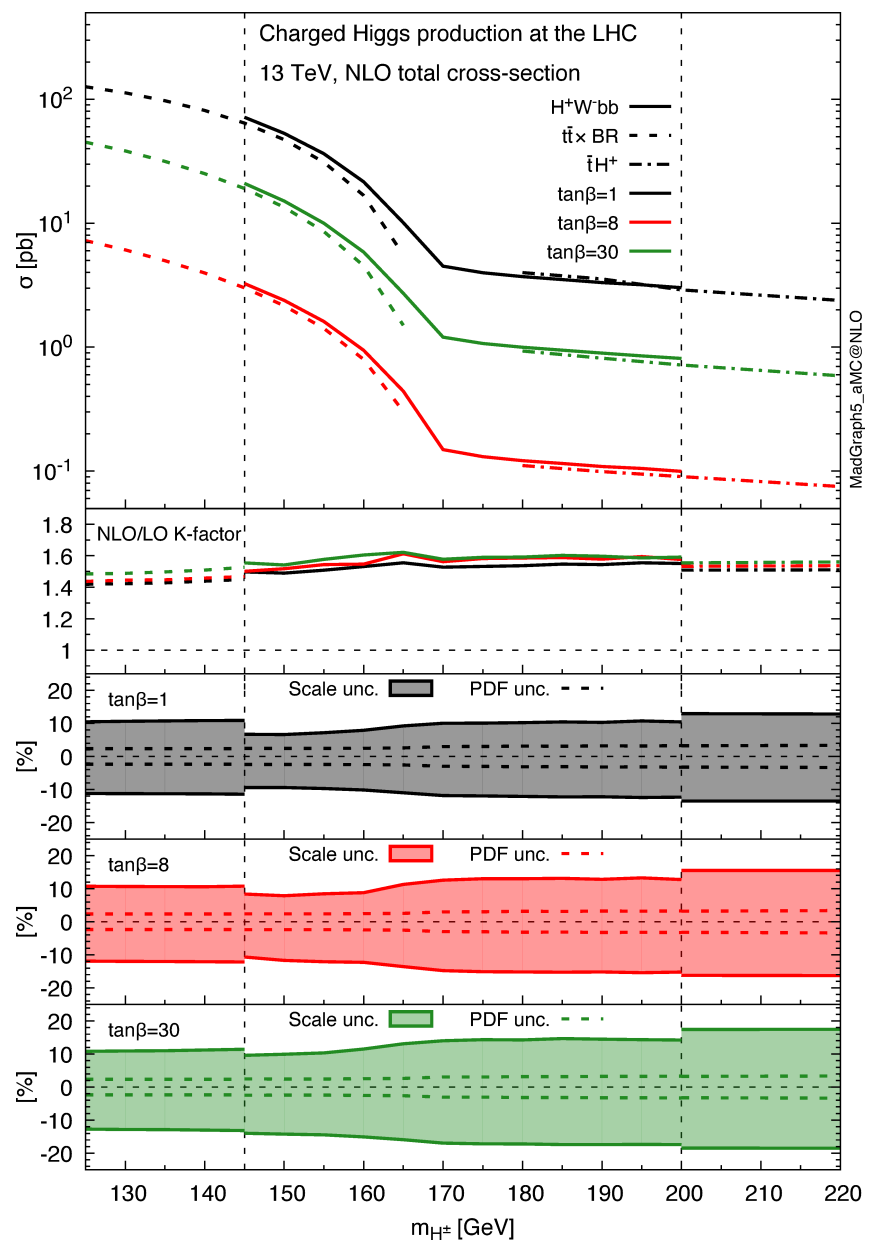

Figure 40: Charged Higgs production cross section in association with top quarks as a function of the charged Higgs mass in the low-, high-and intermediate-mass regimes at the LHC for $\sqrt{s}=13$ TeV. The upper plot shows the central predictions in all three regimes for three values of tgß. The middle plot exhibits the NLO K factor defined as the ratio between the NLO and LO cross sections. The lower plots display the scale-and PDF-uncertainty bands for the three values of tg $\beta$. From Ref. [221].

the same values of the input parameters as for the branching ratios discussed in Section 2.8 and their uncertainties a rigorous analysis has been performed to derive a sophisticated prediction of the central cross section values and their uncertainties. The results are shown in Fig. 41 as a function of the Higgs mass for 13 and $14 \mathrm{TeV}$ c.m. energy at the LHC. The size of the coloured bands represents the individual sums of the theoretical and parametric uncertainties. All production cross sections with results beyond NLO in QCD exhibit a small residual uncertainty in the few-per-cent range. Only the cross sections for $t \bar{t} H, b \bar{b} H$ and $t H$ production develop larger uncertainties due to the problems discussed in the previous sections. The theoretical and parametric uncertainties of each production process have been added in quadrature. The gluon-fusion cross sections can be predicted with a total (Gaussian) uncertainty of about 5\%, the vector-boson-fusion and WH Higgs-strahlung channels with less than 3\% uncertainty and the $Z H$ Higgs-strahlung channel with about $4 \%$ uncertainty due to the novel loop contributions from $g g \rightarrow Z H$ as discussed in Section 3.3.1. The uncertainties of $t \bar{t} H$ production amount to about $10-15 \%$, for $s$ - and $t$-channel $t H$ production to about $15-20 \%$ and for $b \bar{b} H$ production to about $20-25 \%$. Fig. 42 shows the energy dependence of the Higgs production cross sections for a Higgs mass $M_{H}=125 \mathrm{GeV}$. It is visible that all cross sections develop a similar rising slope apart from $t \bar{t} H$ and $t H$ that grow stronger due to the larger phase-space suppression for smaller energies. 

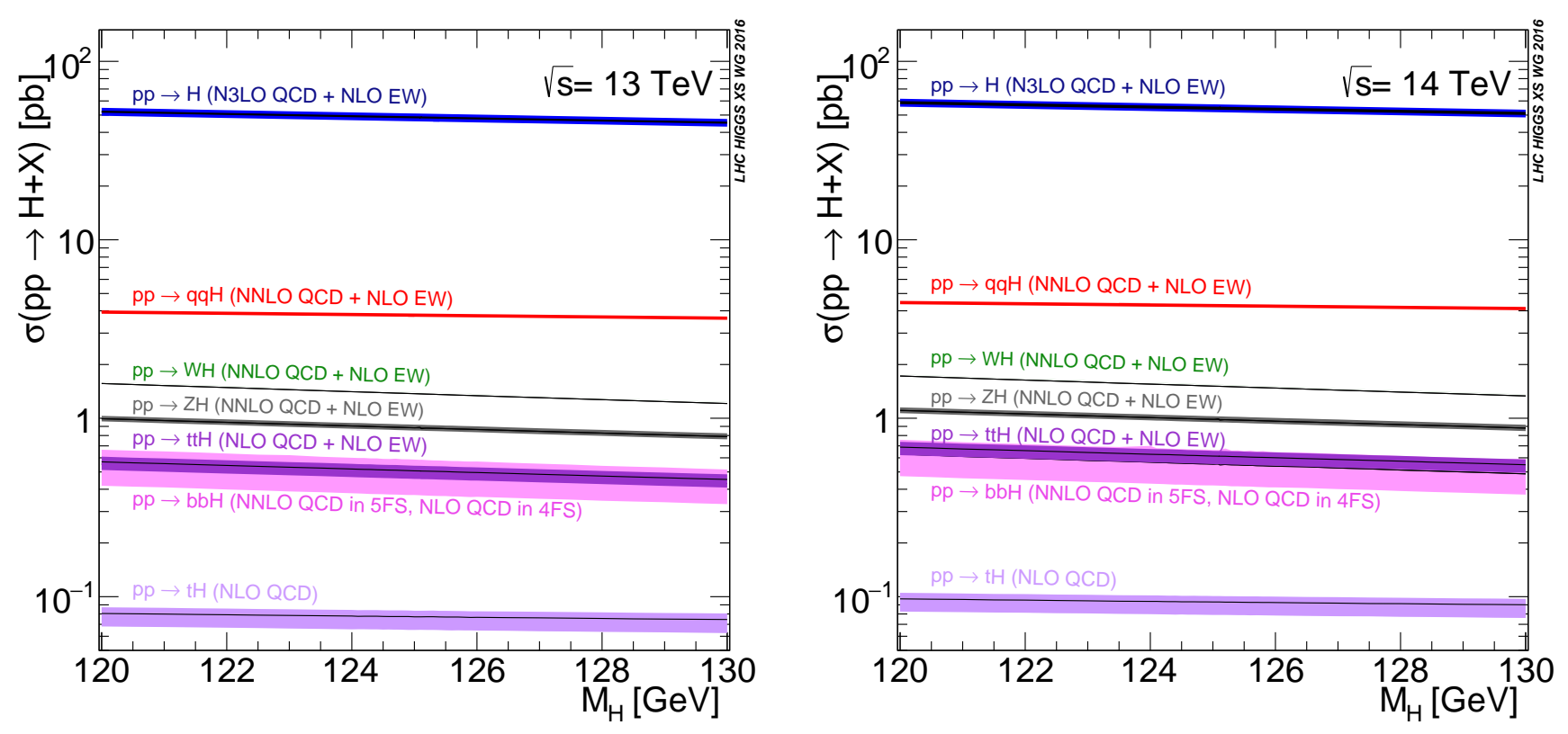

Figure 41: Higgs boson production cross sections as a function of the Higgs mass for 13 and 14 TeV c.m. energy at the LHC including the most up-to-date higher-order corrections as indicated at the shown cross section bands. The size of the bands reflects the total estimated theoretical uncertainties. From Ref. [51] (page 275).

\subsubsection{Minimal supersymmetric extension}

An analogous update of the production cross sections as for the SM case has been made also for the MSSM Higgs-boson production cross sections. The public code SusHi [151] has been used as the preferred choice since this includes the full NLO QCD corrections to the gluon-fusion cross section and the NNLO QCD corrections in the heavy top-quark limit for the top-loop contributions 12 . Moreover, electroweak corrections originating from light-fermion loops [79] are taken into account in this program. For the $b \bar{b} \Phi$ production cross section SUSHI contains the NNLO QCD-corrected 5FS-cross section that agrees with the 4 FS results within about $20 \%$ for the adopted scale choices [51]. SUSY-QCD corrections are included in the heavy SUSY-particle limit for the gluon-fusion process and in the $\Delta_{b}$ approximation for $b \bar{b} \phi$ production. The compiled cross sections within the $m_{h}^{\text {mod }+}$ scenario [137] are shown in Fig. 43 for two values of $\operatorname{tg} \beta$. The left plot exhibits the dominance of the gluon-fusion process for smaller values of $\operatorname{tg} \beta$ and the right one the dominance of $b \bar{b} \phi$ production for large values of $\operatorname{tg} \beta$. Uncertainties have not been added to these results, but have been analyzed in detail in Ref. [231].

\subsection{Higgs boson pair production}

\subsubsection{Standard Model}

Higgs-boson pair production is the first process that allows to obtain direct access to the trilinear self-coupling of the Higgs boson and paves the way to the Higgs potential at the origin of electroweak symmetry breaking 13 . Higgs boson pairs are dominantly produced in the gluon-fusion process and to a lesser extent in vector-boson fusion, double Higgs-strahlung and double Higgs bremsstrahlung off top quarks, see Fig. 44. The production cross sections are shown as a function of the collider energy for a Higgs mass $M_{H}=125 \mathrm{GeV}$ in Fig. 45. Gluon fusion dominates the production of Higgs-boson pairs

\footnotetext{
${ }^{12}$ Meanwhile also the $\mathrm{N}^{3} \mathrm{LO}$ QCD corrections have been included in SusHI [151].

${ }^{13}$ Additional indirect sensitivity to the trilinear Higgs coupling is provided by the single-Higgs production processes due to its contribution to the electroweak corrections [232.
} 


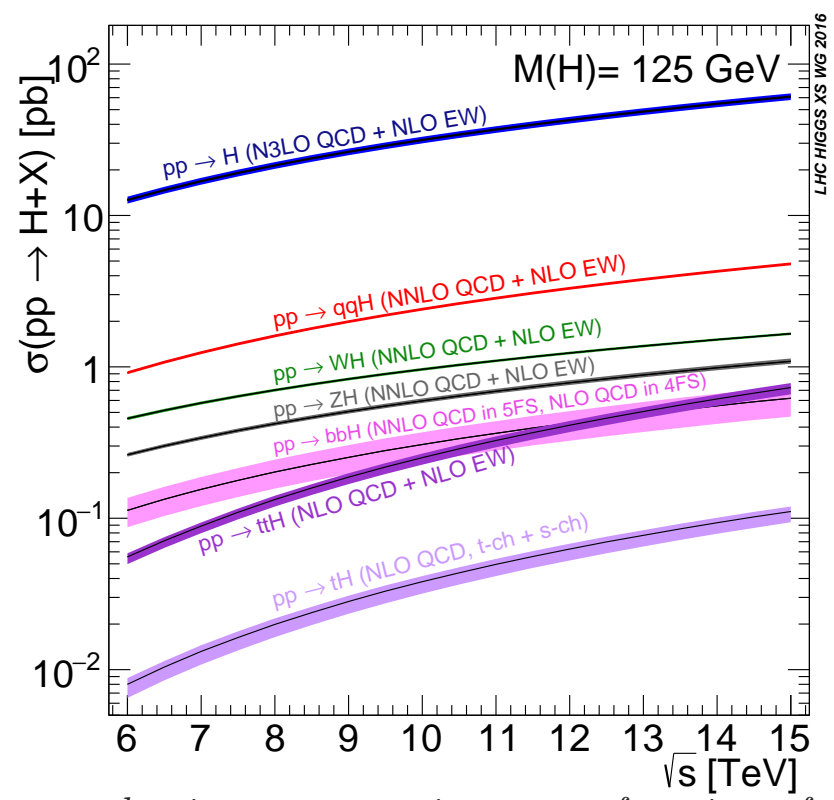

Figure 42: Higgs boson production cross sections as a function of the c.m. energy at the LHC for a Higgs mass $M_{H}=125$ GeV including the most up-to-date higher-order corrections as indicated at the shown cross section bands. The size of the bands reflects the total estimated theoretical uncertainties. From Ref. [51] (page 276).

by more than an order of magnitude, while the production modes roughly follow the pattern of singleHiggs boson production. Since the diagrams involving the trilinear Higgs coupling are only a subset for each process the sensitivity to the trilinear Higgs coupling is reduced compared to the size of the individual cross sections. The sensitivities of the individual production cross sections to the trilinear Higgs coupling are shown in Fig. 45. The locations of the minima of the cross sections in terms of the value of $\lambda$ are different for the production mechanisms [233].

Gluon Fusion. The gluon-fusion process is mediated by top- and to a lesser extent bottom-quark loops. There are box- and triangle contributions at LO, see Fig. 44a. The box contributions are dominant with a destructive interference to the triangle diagrams [235]. The sensitivity to the trilinear Higgs coupling follows the rough behaviour $\Delta \sigma / \sigma \sim-\Delta \lambda / \lambda$ so that the uncertainties of the production cross section are immediately translated to the uncertainties of the extraction of the trilinear selfcoupling.

In the past the NLO QCD corrections have been determined in the limit of heavy top quarks [236]. Similar to the single Higgs case they increase the cross section by up to $100 \%$. Due to the fact that the invariant mass of the final-state Higgs-boson pair is much larger than in the single-Higgs case the heavy top-quark limit is expected to work less reliable in the Higgs-pair case. The first attempt to estimate finite top-mass effects at NLO was by means of a systematic heavy-top expansion of the inclusive cross section [237] that gave an estimate of about $10 \%$ for the finite top-mass effects beyond LO. The second attempt kept the virtual corrections in the heavy-top limit but included the real corrections exactly [238. This resulted in a 10\%-decrease of the NLO cross section. Very recently the full NLO calculation has been completed by numerical methods implying a decrease of the total cross section by about $14 \%$ at the LHC for a c.m. energy of $13 \mathrm{TeV}$ [239] so that the heavy-top limit works still reasonably well for the total cross section. For large invariant Higgs-pair masses the finite mass effects can reach a level of $-20 \%$. The NLO result has been extended by the NNLO QCD corrections in the heavy topquark limit that lead to a rise of the total cross section by about 20\% [240]. Within a heavy top-quark expansion NNLO top mass effects have been estimated to be at the 5\%-level [241]. Finally a NNLL soft 

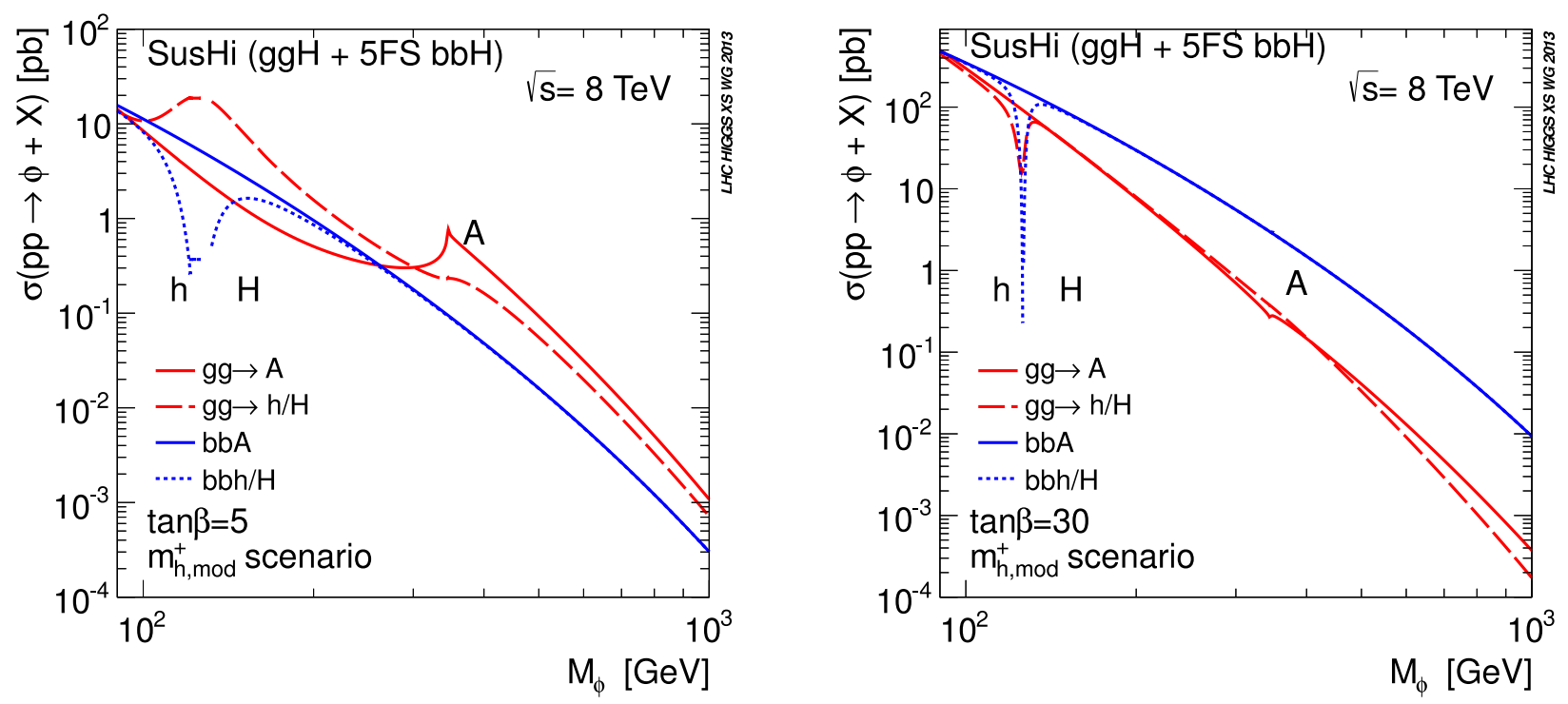

Figure 43: Neutral MSSM Higgs production cross sections at the LHC for a c.m. energy $\sqrt{s}=8$ TeV as functions of the corresponding Higgs mass within the $m_{h}^{\bmod +}$ scenario [137] for two values of tg $\beta$ obtained by SusHi [151]. From Ref. [66].

and collinear gluon resummation has been added which adds a contribution of 5-10\% beyond NNLO 242. Including the most up-to-date theoretical status for the prediction of the total cross section the theoretical uncertainties due to the scale dependence is reduced to about $5 \%$. Together with the $\mathrm{PDF}+\alpha_{s}$ uncertainties one obtains a total uncertainty of about $10 \%$ for the total cross section at the LHC [51]. Recently a public code has been constructed for fully exclusive Higgs-boson pair production via gluon fusion including NNLO QCD corrections at parton level [243].

Vector-boson Fusion. Higgs-boson pair production via vector-boson fusion is dominated by $t$ channel contributions as in the single-Higgs case, see Fig. 44b. The NLO QCD corrections can be taken from deep inelastic lepton-nucleon scattering analogous to single-Higgs production [233, 238]. They increase the cross section by about 10\%. Within the same approach the NNLO QCD corrections have been obtained [244]. They range at the per-cent level. The residual theoretical and parametric uncertainties amount to about $3-4 \%$ at the LHC [51].

Double Higgs-strahlung. Double Higgs-strahlung proceeds along the same lines as single-Higgsstrahlung, i.e. the Higgs boson pair is produced in association with a $W$ or $Z$ boson, see Fig. 44k. The NLO and NNLO QCD corrections can be taken over from the corresponding calculation for the Drell-Yan process, since the final state is only weakly interacting [233, 238. The only difference to the Drell-Yan process emerges from the additional loop-mediated $g g \rightarrow Z H H$ process, see Fig. 44k. This has been added to the NNLO QCD corrections. The QCD corrections increase the production cross sections by about 30\%, while the $g g \rightarrow Z H H$ adds another 20-30\% to $Z H H$ production [233]. The residual theoretical uncertainties range at the 3\%-level for $W H H$ production and at the level of $4 \%$ for $Z H H$ production [51]. A fully differential calculation for $W H H$ final states has recently been completed [245]. 
(a)
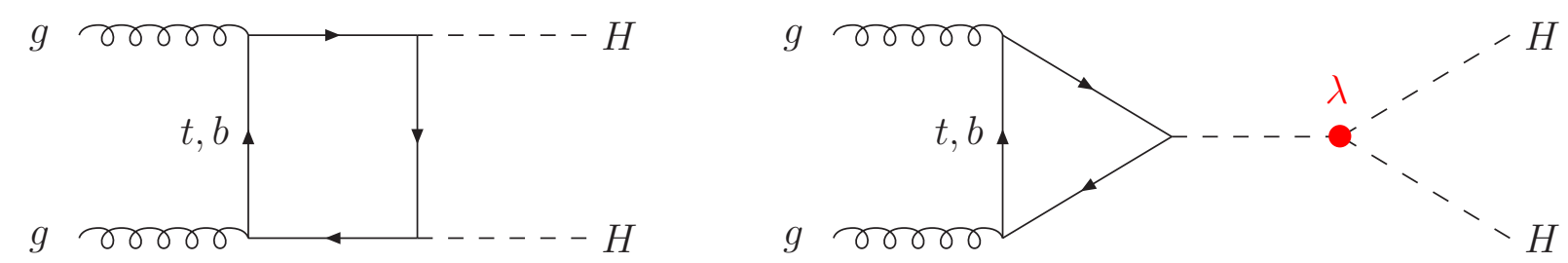

(b)
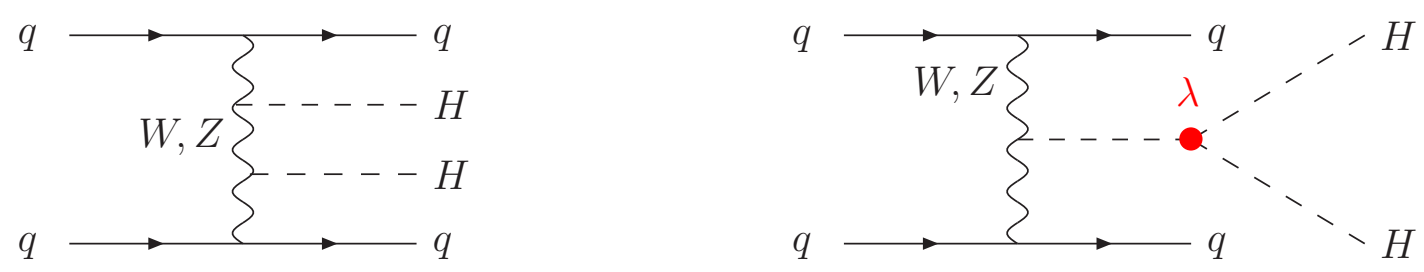

(c)
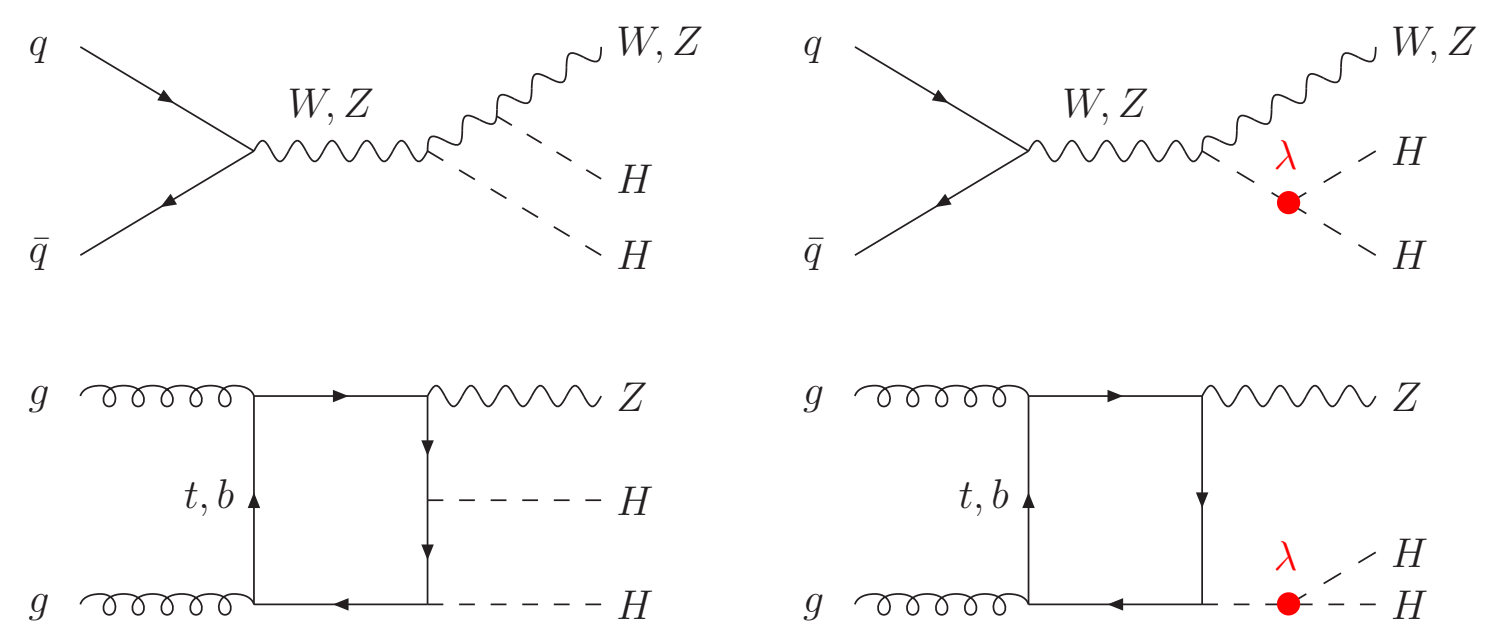

$(d)$
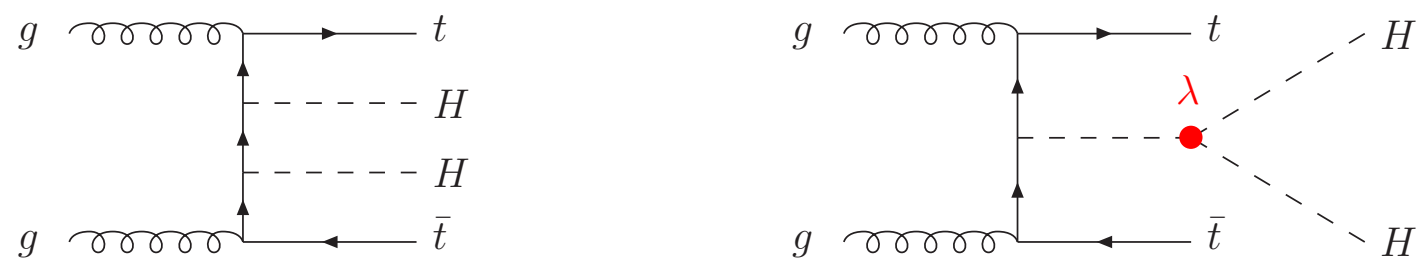

Figure 44: Diagrams contributing to Higgs-boson pair production: (a) gluon fusion, (b) vector-boson fusion, (c) double Higgs-strahlung and (d) double Higgs bremsstrahlung off top quarks. The contribution of the trilinear Higgs coupling is marked in red. 

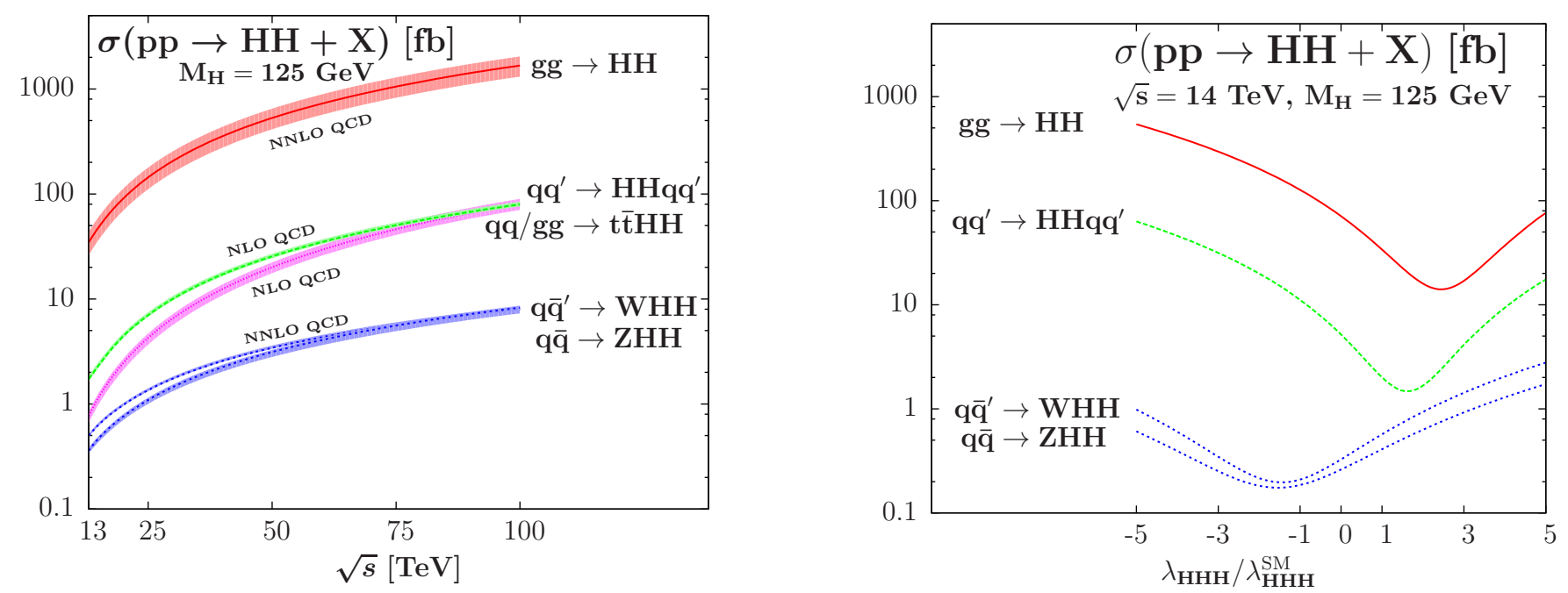

Figure 45: Left: Higgs-boson pair production cross sections as a function of the c.m. energy at the LHC for a Higgs mass $M_{H}=125 \mathrm{GeV}$. The bands represent the theoretical uncertainties. From Ref. 234]. Right: Dependence of the production cross sections on the trilinear Higgs coupling $\lambda_{H H H}$. From Ref. [233].

Double Higgs bremsstrahlung off top quarks. Higgs-boson pair production in association with top quarks is generated by analogous diagrams to the single-Higgs case, see Fig. 44d [233, 246]. The NLO QCD corrections have been calculated recently with the MG5_AMC@NLO tool [238]. They modify the total cross section by about $20 \%$ and reduce the scale dependence to a level of less than $5 \%$. The total theoretical and parametric uncertainties for this production process amount to about $5-6 \%$ at the LHC with c.m. energies of 13 and $14 \mathrm{TeV}$ [51].

\subsubsection{Minimal supersymmetric extension}
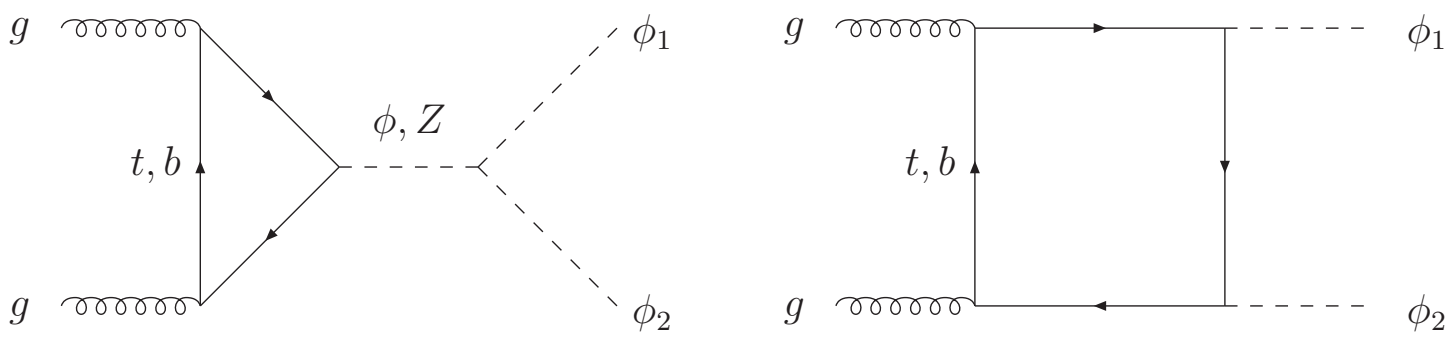

Figure 46: Generic diagrams describing neutral Higgs-boson pair production in gluon-gluon collisions $\left(\phi, \phi_{i}=h, H, A\right)$.

In the MSSM there are several possible neutral Higgs-pair final states. The gluon-fusion mechanism yields $h h, h H, H H, h A, H A, A A$ final states that emerge from analogous diagrams as in the SM-Higgs case. In the mixed scalar-pseudoscalar channel, i.e. $g g \rightarrow h / H+A$, off-shell $Z$-boson exchange contributes to the $s$-channel diagrams, too, see Fig. 46. However, these final states are dominated by Drell-Yan production mechanisms, see Fig. 47. Since the QCD corrections to MSSM Higgs pair production via gluon fusion are only known in the heavy-top-quark limit so far, reliable NLO predictions of the corresponding cross sections are only possible for small values of $\operatorname{tg} \beta$ where the top loops provide the dominant contributions and for not too large external Higgs masses. The QCD corrections are large 
and positive, increasing the cross sections by up to 100\% [236]. The SUSY-QCD corrections are known in the limit of large SUSY-particle and top masses [247] which is also reliable for smaller values of $\operatorname{tg} \beta$ where the bottom-loop contributions are suppressed. The impact of SUSY-contributions starts to be sizeable for squark mass below about $1 \mathrm{TeV}$. The MSSM cross sections for the gluon-fusion processes range below $10 \mathrm{fb}$ in all regions where none of the Higgs bosons involved in the $s$-channel becomes resonant. However, for small values of $\operatorname{tg} \beta$ and below the $t \bar{t}$-threshold there are sizeable regions where the heavy scalar Higgs boson can become resonant and decays into $h h$ final states, $g g \rightarrow H \rightarrow h h$. In these regions the cross sections become large, since the dominant piece factorizes into the single heavy-scalar Higgs production cross section and the branching ratio of the $H \rightarrow h h$ decay [236].

Mixed scalar-pseudoscalar Higgs boson production is dominated by the Drell-Yan process $q \bar{q} \rightarrow$ $h / H+A$. The QCD corrections can be translated from the corresponding Drell-Yan process and are of moderate size [236]. The cross sections for these processes can reach the level of about $100 \mathrm{fb}$.

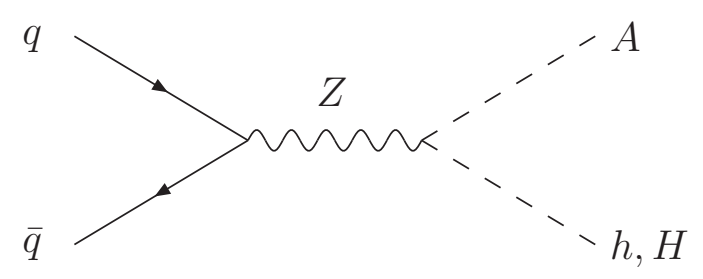

Figure 47: Diagram contributing to $q \bar{q} \rightarrow A h, A H$ at lowest order.

\section{Summary}

In this review the decay widths and branching ratios of SM and MSSM Higgs bosons have been discussed. All relevant higher order corrections, which are dominated by QCD corrections, have been summarized according to the present state of the art. At the LHC the SM Higgs particle is produced predominantly by gluon fusion $g g \rightarrow H$, followed by vector-boson fusion $V V \rightarrow H(V=W, Z)$ and, to a lesser extent, Higgs-strahlung off vector bosons, $V^{*} \rightarrow V H$, and top quarks, $g g / q \bar{q} \rightarrow t \bar{t} H$. The cross sections of these production channels have been described including all known QCD and electroweak corrections, which are important in particular for the dominant gluon-fusion mechanism where they increase the production cross section by about a factor of two. The QCD corrections to the subleading production processes are moderate in case of the electroweak vector-boson fusion and Higgs-strahlung but also for $t \bar{t} H$ production, since in the latter process the threshold region is strongly phase-space suppressed. For vector-boson fusion and Higgs-strahlung the electroweak corrections are of similar size as the QCD ones. The partial decay widths are all known with per-cent or sub-per-cent precision.

In the MSSM the neutral Higgs bosons will mainly be produced via gluon fusion $g g \rightarrow \Phi$. However, through the enhanced $b$ quark couplings, Higgs bremsstrahlung off $b$ quarks, $g g / q \bar{q} \rightarrow b \bar{b} \Phi$, will dominate for large $\operatorname{tg} \beta$. All other Higgs production mechanisms, i.e. vector-boson fusion and Higgs-strahlung off vector bosons or $t \bar{t}$ pairs, will be less important than in the SM. The potentially large SUSYQCD and -electroweak corrections to the bottom Yukawa coupling constitute a major ingredient for accurate predictions of production and decay processes involving the bottom Yukawa coupling. For configurations with on-shell bottom quarks the leading $\Delta_{b}$ terms introduced in Section 1.3 provide an excellent approximation to the full NLO results, while cases with far off-shell bottom quarks as e.g. the gluon-fusion processes $g g \rightarrow h / H / A$ or the reverse gluonic Higgs decays develop sizeable corrections 
beyond the $\Delta_{b}$-approximation for the genuine supersymmetric part of the radiative corrections.

\section{Acknowledgements}

I am grateful to J. Baglio, S. Dittmaier and M. M. Mühlleitner for reading the manuscript and valuable comments and discussions.

\section{References}

[1] B. A. Kniehl, Phys. Rept. 240 (1994) 211; A. Djouadi, Phys. Rept. 457 (2008) 1 and Phys. Rept. 459 (2008) 1; S. Dittmaier and M. Schumacher, Prog. Part. Nucl. Phys. 70 (2013) 1; J. Baglio, A. Djouadi and J. Quevillon, Rept. Prog. Phys. 79 (2016) no.11, 116201.

[2] M. Spira, Fortsch. Phys. 46 (1998) 203.

[3] G. Aad et al. [ATLAS Collaboration], Phys. Lett. B716 (2012) 1; S. Chatrchyan et al. [CMS Collaboration], Phys. Lett. B716 (2012) 30.

[4] G. Aad et al. [ATLAS and CMS Collaborations], JHEP 1608 (2016) 045.

[5] P. W. Higgs, Phys. Lett. 12 (1964) 132, Phys. Rev. Lett. 13 (1964) 508 and Phys. Rev. 145 (1966) 1156; F. Englert and R. Brout, Phys. Rev. Lett. 13 (1964) 321; G. S. Guralnik, C. R. Hagen and T. W. Kibble, Phys. Rev. Lett. 13 (1964) 585; T. W. B. Kibble, Phys. Rev. 155 (1967) 1554.

[6] G. 't Hooft, Nucl. Phys. B 35 (1971) 167; G. 't Hooft and M. J. G. Veltman, Nucl. Phys. B 44 (1972) 189.

[7] C. H. Llewellyn Smith, Phys. Lett. 46B (1973) 233; J. M. Cornwall, D. N. Levin and G. Tiktopoulos, Phys. Rev. D 10 (1974) 1145 Erratum: [Phys. Rev. D 11 (1975) 972]; B. W. Lee, C. Quigg and H. B. Thacker, Phys. Rev. Lett. 38 (1977) 883 and Phys. Rev. D 16 (1977) 1519.

[8] N. Cabibbo, L. Maiani, G. Parisi and R. Petronzio, Nucl. Phys. B 158 (1979) 295; M. S. Chanowitz, M. A. Furman and I. Hinchliffe, Phys. Lett. 78B (1978) 285; R. A. Flores and M. Sher, Phys. Rev. D 27 (1983) 1679; M. Lindner, Z. Phys. C 31 (1986) 295; A. Hasenfratz, K. Jansen, C. B. Lang, T. Neuhaus and H. Yoneyama, Phys. Lett. B 199 (1987) 531; J. Kuti, L. Lin and Y. Shen, Phys. Rev. Lett. 61 (1988) 678; M. Lüscher and P. Weisz, Nucl. Phys. B 318 (1989) 705; M. Sher, Phys. Rept. 179 (1989) 273 and Phys. Lett. B 317 (1993) 159 Addendum: [Phys. Lett. B 331 (1994) 448]; G. Altarelli and G. Isidori, Phys. Lett. B 337 (1994) 141; J. A. Casas, J. R. Espinosa and M. Quiros, Phys. Lett. B 342 (1995) 171.

[9] G. Degrassi, S. Di Vita, J. Elias-Miro, J. R. Espinosa, G. F. Giudice, G. Isidori and A. Strumia, JHEP 1208 (2012) 098; D. Buttazzo, G. Degrassi, P. P. Giardino, G. F. Giudice, F. Sala, A. Salvio and A. Strumia, JHEP 1312 (2013) 089.

[10] J. R. Espinosa and M. Quiros, Phys. Lett. B 353 (1995) 257.

[11] E. Gildener and S. Weinberg, Phys. Rev. D 13 (1976) 3333; S. Weinberg, Phys. Rev. D 13 (1976) 974 and Phys. Rev. D 19 (1979) 1277; L. Susskind, Phys. Rev. D 20 (1979) 2619.

[12] Y. A. Golfand and E. P. Likhtman, JETP Lett. 13 (1971) 323 [Pisma Zh. Eksp. Teor. Fiz. 13 (1971) 452]; D. V. Volkov and V. P. Akulov, Phys. Lett. 46B (1973) 109; J. Wess and B. Zumino, Nucl. Phys. B 70 (1974) 39. 
[13] For reviews on supersymmetric theories, see P. Fayet and S. Ferrara, Phys. Rept. 32 (1977) 249; H. P. Nilles, Phys. Rept. 110 (1984) 1; R. Barbieri, Riv. Nuovo Cim. 11N4 (1988) 1; H. E. Haber and G. L. Kane, Phys. Rept. 117 (1985) 75.

[14] N. Arkani-Hamed, A. G. Cohen and H. Georgi, Phys. Lett. B 513 (2001) 232; N. Arkani-Hamed, A. G. Cohen, T. Gregoire and J. G. Wacker, JHEP 0208 (2002) 020; N. Arkani-Hamed, A. G. Cohen, E. Katz, A. E. Nelson, T. Gregoire and J. G. Wacker, JHEP 0208 (2002) 021.

[15] N. Arkani-Hamed, S. Dimopoulos and G. R. Dvali, Phys. Lett. B 429 (1998) 263; I. Antoniadis, N. Arkani-Hamed, S. Dimopoulos and G. R. Dvali, Phys. Lett. B 436 (1998) 257; L. Randall and R. Sundrum, Phys. Rev. Lett. 83 (1999) 3370 and Phys. Rev. Lett. 83 (1999) 4690.

[16] H. M. Georgi, S. L. Glashow, M. E. Machacek and D. V. Nanopoulos, Phys. Rev. Lett. 40 (1978) 692.

[17] D. R. T. Jones and S. T. Petcov, Phys. Lett. 84B (1979) 440; R. N. Cahn and S. Dawson, Phys. Lett. 136B (1984) 196 Erratum: [Phys. Lett. 138B (1984) 464]; K. I. Hikasa, Phys. Lett. 164B (1985) 385 Erratum: [Phys. Lett. B 195 (1987) 623]; G. Altarelli, B. Mele and F. Pitolli, Nucl. Phys. B 287 (1987) 205.

[18] S. L. Glashow, D. V. Nanopoulos and A. Yildiz, Phys. Rev. D 18 (1978) 1724; Z. Kunszt, Z. Trocsanyi and W. J. Stirling, Phys. Lett. B 271 (1991) 247.

[19] R. Raitio and W.W. Wada, Phys. Rev. D19 (1979) 941; J. N. Ng and P. Zakarauskas, Phys. Rev. D29 (1984) 876; Z. Kunszt, Nucl. Phys. B247 (1984) 339; J. F. Gunion, Phys. Lett. B261 (1991) 510-517; W. J. Marciano and F. E. Paige, Phys. Rev. Lett. 66 (1991) $2433-2435$.

[20] J. M. Butterworth, A. R. Davison, M. Rubin and G. P. Salam, Phys. Rev. Lett. 100 (2008) 242001.

[21] D. L. Rainwater, D. Zeppenfeld and K. Hagiwara, Phys. Rev. D 59 (1998) 014037; T. Plehn, D. L. Rainwater and D. Zeppenfeld, Phys. Lett. B 454 (1999) 297.

[22] E. Witten, Phys. Lett. 105B (1981) 267.

[23] S. Dimopoulos, S. Raby and F. Wilczek, Phys. Rev. D 24 (1981) 1681; L. E. Ibanez and G. G. Ross, Phys. Lett. 105B (1981) 439.

[24] L. E. Ibanez and G. G. Ross, Phys. Lett. 110B (1982) 215.

[25] H. Goldberg, Phys. Rev. Lett. 50 (1983) 1419 Erratum: [Phys. Rev. Lett. 103 (2009) 099905]; J. R. Ellis, J. S. Hagelin, D. V. Nanopoulos, K. A. Olive and M. Srednicki, Nucl. Phys. B 238 (1984) 453 .

[26] S. Dimopoulos and H. Georgi, Nucl. Phys. B 193 (1981) 150.

[27] P. Fayet, Nucl. Phys. B 90 (1975) 104, Phys. Lett. B 64 (1976) 159 and Phys. Lett. B 69 (1977) 489; N. Sakai, Z. Phys. C 11 (1981) 153; K. Inoue, A. Kakuto, H. Komatsu and S. Takeshita, Prog. Theor. Phys. 67 (1982) 1889, Prog. Theor. Phys. 68 (1982) 927 [Erratum-ibid. 70 (1983) 330] and Prog. Theor. Phys. 71 (1984) 413.

[28] See e.g. G. Degrassi, S. Heinemeyer, W. Hollik, P. Slavich and G. Weiglein, Eur. Phys. J. C28 (2003) 133 . 
[29] F. Zwirner, In *Saariselkae 1991, Proceedings, Physics and experiments with linear colliders, vol. 1*309-351 and CERN Geneva - TH. 6357 [hep-ph/9203204].

[30] S. P. Martin, Phys. Rev. D75 (2007) 055005; R. V. Harlander, P. Kant, L. Mihaila and M. Steinhauser, Phys. Rev. Lett. 100 (2008) 191602, Erratum ibid. 101 (2008) 039901.

[31] P. Draper, G. Lee and C. E. M. Wagner, Phys. Rev. D 89 (2014) no.5, 055023; E. Bagnaschi, G. F. Giudice, P. Slavich and A. Strumia, JHEP 1409 (2014) 092; K. Cheung, R. Huo, J. S. Lee and Y. L. Sming Tsai, JHEP 1504 (2015) 151; J. Pardo Vega and G. Villadoro, JHEP 1507 (2015) 159; G. Lee and C. E. M. Wagner, Phys. Rev. D 92 (2015) no.7, 075032; H. Bahl and W. Hollik, Eur. Phys. J. C 76 (2016) no.9, 499.

[32] M. Frank, T. Hahn, S. Heinemeyer, W. Hollik, H. Rzehak and G. Weiglein, JHEP 0702 (2007) 047; M. Beneke, P. Ruiz-Femenia and M. Spinrath, JHEP 0901 (2009) 031.

[33] V.D. Barger, M.S. Berger, A.L. Stange and R.J.N. Phillips, Phys. Rev. D45 (1992) 4128; W. Hollik and S. Peñaranda, Eur. Phys. J. C23 (2002) 163; A. Dobado, M.J. Herrero, W. Hollik and S. Peñaranda, Phys. Rev. D66 (2002) 095016.

[34] M. Brucherseifer, R. Gavin and M. Spira, Phys. Rev. D 90 (2014) no.11, 117701; M. Brucherseifer, PhD thesis, ETH Zürich, 2012.

[35] L. J. Hall, R. Rattazzi and U. Sarid, Phys. Rev. D 50, 7048 (1994); R. Hempfling, Phys. Rev. D 49, 6168 (1994); M. Carena, M. Olechowski, S. Pokorski and C. E. M. Wagner, Nucl. Phys. B 426 (1994) 269; D. M. Pierce, J. A. Bagger, K. T. Matchev and R.-J. Zhang, Nucl. Phys. B 491, 3 (1997); J. Guasch, W. Hollik and S. Peñaranda, Phys. Lett. B 515 (2001) 367; G. D'Ambrosio, G. F. Giudice, G. Isidori and A. Strumia, Nucl. Phys. B 645, 155 (2002); A. J. Buras, P. H. Chankowski, J. Rosiek and L. Slawianowska, Nucl. Phys. B 659, 3 (2003); V. Barger, H. E. Logan and G. Shaughnessy, Phys. Rev. D 79, 115018 (2009); N. D. Christensen, T. Han and S. Su, Phys. Rev. D 85, 115018 (2012).

[36] M. S. Carena, D. Garcia, U. Nierste and C. E. M. Wagner, Nucl. Phys. B 577, 88 (2000).

[37] J. Guasch, P. Häfliger and M. Spira, Phys. Rev. D 68, 115001 (2003).

[38] D. Noth and M. Spira, Phys. Rev. Lett. 101, 181801 (2008) and JHEP 1106, 084 (2011).

[39] L. Mihaila and C. Reisser, JHEP 1008, 021 (2010); A. Crivellin and C. Greub, Phys. Rev. D 87 (2013) 015013 Erratum: [Phys. Rev. D 87 (2013) 079901].

[40] J. R. Ellis, M. K. Gaillard and D. V. Nanopoulos, Nucl. Phys. B 106 (1976) 292.

[41] L. Resnick, M. K. Sundaresan and P. J. S. Watson, Phys. Rev. D 8 (1973) 172.

[42] E. Braaten and J. P. Leveille, Phys. Rev. D 22 (1980) 715; N. Sakai, Phys. Rev. D 22 (1980) 2220; T. Inami and T. Kubota, Nucl. Phys. B 179 (1981) 171; M. Drees and K. I. Hikasa, Phys. Lett. B 240 (1990) 455 Erratum: [Phys. Lett. B 262 (1991) 497] and Phys. Rev. D 41 (1990) 1547.

[43] S. G. Gorishnii, A. L. Kataev and S. A. Larin, Sov. J. Nucl. Phys. 40 (1984) 329 [Yad. Fiz. 40 (1984) 517]; S. G. Gorishnii, A. L. Kataev, S. A. Larin and L. R. Surguladze, Mod. Phys. Lett. A 5 (1990) 2703 and Phys. Rev. D 43 (1991) 1633; A. L. Kataev and V. T. Kim, Mod. Phys. Lett. A 9 (1994) 1309; L. R. Surguladze, Phys. Lett. B 341 (1994) 60; K. G. Chetyrkin, Phys. Lett. B 390 (1997) 309; K. Melnikov, Phys. Rev. D 53 (1996) 5020. 
[44] K. G. Chetyrkin and A. Kwiatkowski, Nucl. Phys. B 461 (1996) 3; S. A. Larin, T. van Ritbergen and J. A. M. Vermaseren, Phys. Lett. B 362 (1995) 134.

[45] J. Fleischer and F. Jegerlehner, Phys. Rev. D 23 (1981) 2001.

[46] D. Y. Bardin, B. M. Vilensky and P. K. Khristova, Sov. J. Nucl. Phys. 53 (1991) 152 [Yad. Fiz. 53 (1991) 240]; A. Dabelstein and W. Hollik, Z. Phys. C 53 (1992) 507; B. A. Kniehl, Nucl. Phys. B 376 (1992) 3.

[47] A. Djouadi, D. Haidt, B. A. Kniehl, P. M. Zerwas and B. Mele, In *Munich/Annecy/Hamburg 1991, Proceedings, $e^{+} e^{-}$collisions at $500 \mathrm{GeV}$, pt. A* 11-30.

[48] B. A. Kniehl and M. Spira, Nucl. Phys. B 432 (1994) 39; A. Kwiatkowski and M. Steinhauser, Phys. Lett. B 338 (1994) 66 Erratum: [Phys. Lett. B 342 (1995) 455].

[49] K. G. Chetyrkin, B. A. Kniehl and M. Steinhauser, Phys. Rev. Lett. 78 (1997) 594.

[50] L. Mihaila, B. Schmidt and M. Steinhauser, Phys. Lett. B 751 (2015) 442.

[51] D. de Florian et al. [LHC Higgs Cross Section Working Group Collaboration], arXiv:1610.07922 [hep-ph].

[52] C. S. Li and R. J. Oakes, Phys. Rev. D 43 (1991) 855; A. Mendez and A. Pomarol, Phys. Lett. B 252 (1990) 461.

[53] A. Djouadi and P. Gambino, Phys. Rev. D 51 (1995) 218 Erratum: [Phys. Rev. D 53 (1996) 4111].

[54] A. Djouadi, J. Kalinowski and M. Spira, Comput. Phys. Commun. 108 (1998) 56; A. Djouadi, M. M. Mühlleitner and M. Spira, Acta Phys. Polon. B 38 (2007) 635.

[55] A. Dabelstein, Nucl. Phys. B 456 (1995) 25; R. A. Jimenez and J. Sola, Phys. Lett. B 389 (1996) 53; J. A. Coarasa Perez, R. A. Jimenez and J. Sola, Phys. Lett. B 389 (1996) 312.

[56] A. Djouadi, J. Kalinowski and P. M. Zerwas, Z. Phys. C 70 (1996) 435; S. Moretti and W. J. Stirling, Phys. Lett. B 347 (1995) 291 Erratum: [Phys. Lett. B 366 (1996) 451].

[57] B. A. Kniehl, Nucl. Phys. B 352 (1991) 1; D. Y. Bardin, P. K. Khristova and B. M. Vilensky, Sov. J. Nucl. Phys. 54 (1991) 833 [Yad. Fiz. 54 (1991) 1366].

[58] B. A. Kniehl and M. Spira, Z. Phys. C 69 (1995) 77.

[59] B. A. Kniehl and M. Spira, Nucl. Phys. B 443 (1995) 37.

[60] B. A. Kniehl and M. Steinhauser, Phys. Lett. B 365 (1996) 297 and Nucl. Phys. B 454 (1995) 485.

[61] T. G. Rizzo, Phys. Rev. D 22 (1980) 722; W. Y. Keung and W. J. Marciano, Phys. Rev. D 30 (1984) 248.

[62] R. N. Cahn, Rept. Prog. Phys. 52 (1989) 389.

[63] A. Bredenstein, A. Denner, S. Dittmaier and M. M. Weber, Phys. Rev. D 74 (2006) 013004 and JHEP 0702 (2007) 080.

[64] S. Boselli, C. M. Carloni Calame, G. Montagna, O. Nicrosini and F. Piccinini, JHEP 1506 (2015) 023. 
[65] A. Denner, S. Heinemeyer, I. Puljak, D. Rebuzzi and M. Spira, Eur. Phys. J. C 71 (2011) 1753.

[66] S. Heinemeyer et al. [LHC Higgs Cross Section Working Group Collaboration], arXiv:1307.1347 [hep-ph].

[67] W. Hollik and J. H. Zhang, arXiv:1011.6537 [hep-ph] and Phys. Rev. D 84 (2011) 055022.

[68] M. Spira, A. Djouadi, D. Graudenz and P. M. Zerwas, Nucl. Phys. B 453 (1995) 17.

[69] T. Inami, T. Kubota and Y. Okada, Z. Phys. C 18 (1983) 69.

[70] A. Djouadi, M. Spira and P. M. Zerwas, Phys. Lett. B 264 (1991) 440.

[71] K. G. Chetyrkin, B. A. Kniehl and M. Steinhauser, Phys. Rev. Lett. 79 (1997) 353.

[72] P. A. Baikov and K. G. Chetyrkin, Phys. Rev. Lett. 97 (2006) 061803.

[73] M. A. Shifman, A. I. Vainshtein, M. B. Voloshin and V. I. Zakharov, Sov. J. Nucl. Phys. 30 (1979) 711 [Yad. Fiz. 30 (1979) 1368].

[74] M. Spira, JHEP 1610 (2016) 026.

[75] K. G. Chetyrkin, B. A. Kniehl and M. Steinhauser, Nucl. Phys. B 510 (1998) 61.

[76] W. Bernreuther and W. Wetzel, Nucl. Phys. B 197 (1982) 228 Erratum: [Nucl. Phys. B 513 (1998) 758]; S. A. Larin, T. van Ritbergen and J. A. M. Vermaseren, Nucl. Phys. B 438 (1995) 278; Y. Schröder and M. Steinhauser, JHEP 0601 (2006) 051; K. G. Chetyrkin, J. H. Kühn and C. Sturm, Nucl. Phys. B 744 (2006) 121.

[77] B. A. Kniehl and A. V. Kotikov, Phys. Lett. B 642 (2006) 68; R. N. Lee and I. S. Terekhov, JHEP 1101 (2011) 068; B. A. Kniehl, A. V. Kotikov, A. I. Onishchenko and O. L. Veretin, Phys. Rev. Lett. 97 (2006) 042001; K. Chetyrkin, P. Baikov and J. Kühn, PoS LL 2016 (2016) 010.

[78] A. Djouadi and P. Gambino, Phys. Rev. Lett. 73 (1994) 2528; K. G. Chetyrkin, B. A. Kniehl and M. Steinhauser, Nucl. Phys. B 490 (1997) 19.

[79] U. Aglietti, R. Bonciani, G. Degrassi and A. Vicini, Phys. Lett. B 595 (2004) 432 and hep-ph/0610033; G. Degrassi and F. Maltoni, Phys. Lett. B 600 (2004) 255.

[80] S. Actis, G. Passarino, C. Sturm and S. Uccirati, Phys. Lett. B 670 (2008) 12; S. Actis, G. Passarino, C. Sturm and S. Uccirati, Nucl. Phys. B 811 (2009) 182.

[81] S. Dawson, A. Djouadi and M. Spira, Phys. Rev. Lett. 77 (1996) 16.

[82] J. F. Gunion, H. E. Haber, G. L. Kane and S. Dawson, Front. Phys. 80 (2000) 1.

[83] M. Mühlleitner and M. Spira, Nucl. Phys. B 790 (2008) 1; R. Bonciani, G. Degrassi and A. Vicini, JHEP 0711 (2007) 095.

[84] S. Gori and I. Low, JHEP 1309 (2013) 151.

[85] M. Mühlleitner, H. Rzehak and M. Spira, JHEP 0904 (2009) 023.

[86] H. E. Haber and G. L. Kane, Phys. Rept. 117 (1985) 75. 
[87] G. 't Hooft and M. J. G. Veltman, Nucl. Phys. B 44 (1972) 189; P. Breitenlohner and D. Maison, Commun. Math. Phys. 52 (1977) 11.

[88] S. A. Larin, Phys. Lett. B 303 (1993) 113.

[89] M. Spira, A. Djouadi, D. Graudenz and P. M. Zerwas, Phys. Lett. B 318 (1993) 347.

[90] J. G. Körner, D. Kreimer and K. Schilcher, Z. Phys. C 54 (1992) 503; D. Kreimer, hep-ph/9401354.

[91] K. Melnikov, M. Spira and O. I. Yakovlev, Z. Phys. C 64 (1994) 401.

[92] K. G. Chetyrkin, B. A. Kniehl, M. Steinhauser and W. A. Bardeen, Nucl. Phys. B 535 (1998) 3.

[93] S. L. Adler, Phys. Rev. 177 (1969) 2426; J. S. Bell and R. Jackiw, Nuovo Cim. A 60 (1969) 47.

[94] D. G. Sutherland, Nucl. Phys. B 2 (1967) 433; M. J. G. Veltman, Proc. Roy. Soc. A 301 (1967) 107.

[95] S. L. Adler and W. A. Bardeen, Phys. Rev. 182 (1969) 1517.

[96] J. Brod, F. Fugel and B. A. Kniehl, Phys. Rev. D 78 (2008) 011303 and Nucl. Phys. B 807 (2009) 188.

[97] V. V. Sudakov, Sov. Phys. JETP 3 (1956) 65 [Zh. Eksp. Teor. Fiz. 30 (1956) 87].

[98] M. I. Kotsky and O. I. Yakovlev, Phys. Lett. B 418 (1998) 335; R. Akhoury, H. Wang and O. I. Yakovlev, Phys. Rev. D 64 (2001) 113008.

[99] R. V. Harlander and M. Steinhauser, Phys. Lett. B 574 (2003) 258 and JHEP 0409 (2004) 066.

[100] G. Degrassi and P. Slavich, Nucl. Phys. B 805 (2008) 267; G. Degrassi, S. Di Vita and P. Slavich, Eur. Phys. J. C 72 (2012) 2032.

[101] R. V. Harlander and F. Hofmann, JHEP 0603 (2006) 050; R. V. Harlander, F. Hofmann and H. Mantler, JHEP 1102 (2011) 055;

[102] G. Degrassi, S. Di Vita and P. Slavich, JHEP 1108 (2011) 128.

[103] G. Degrassi and P. Slavich, JHEP 1011 (2010) 044.

[104] C. Anastasiou, S. Beerli and A. Daleo, Phys. Rev. Lett. 100 (2008) 241806.

[105] M. Mühlleitner, H. Rzehak and M. Spira, PoS RADCOR 2009 (2010) 043 and DESY-PROC2010-01.

[106] M. Carena, S. Heinemeyer, C. E. M. Wagner and G. Weiglein, Eur. Phys. J. C 26 (2003) 601.

[107] H.-Q. Zheng and D.-D. Wu, Phys. Rev. D 42 (1990) 3760; A. Djouadi, M. Spira, J. J. van der Bij and P. M. Zerwas, Phys. Lett. B 257 (1991) 187; S. Dawson and R. P. Kauffman, Phys. Rev. D 47 (1993) 1264; A. Djouadi, M. Spira and P. M. Zerwas, Phys. Lett. B 311 (1993) 255; K. Melnikov and O. I. Yakovlev, Phys. Lett. B 312 (1993) 179; M. Inoue, R. Najima, T. Oka and J. Saito, Mod. Phys. Lett. A 9 (1994) 1189; J. Fleischer, O. V. Tarasov and V. O. Tarasov, Phys. Lett. B 584 (2004) 294. 
[108] R. Harlander and P. Kant, JHEP 0512 (2005) 015; C. Anastasiou, S. Beerli, S. Bucherer, A. Daleo and Z. Kunszt, JHEP 0701 (2007) 082; U. Aglietti, R. Bonciani, G. Degrassi and A. Vicini, JHEP 0701 (2007) 021.

[109] M. Steinhauser, In *Tegernsee 1996, The Higgs puzzle* 177-185 hep-ph/9612395.

[110] C. Sturm, Eur. Phys. J. C 74 (2014) 8, 2978.

[111] P. Maierhöfer and P. Marquard, Phys. Lett. B 721 (2013) 131.

[112] A. Djouadi, P. Gambino and B. A. Kniehl, Nucl. Phys. B 523 (1998) 17; G. Degrassi and F. Maltoni, Nucl. Phys. B 724 (2005) 183; G. Passarino, C. Sturm and S. Uccirati, Phys. Lett. B 655 (2007) 298; S. Actis, G. Passarino, C. Sturm and S. Uccirati, Nucl. Phys. B 811 (2009) 182.

[113] A. Djouadi, V. Driesen, W. Hollik and J. I. Illana, Eur. Phys. J. C 1 (1998) 149.

[114] R. N. Cahn, M. S. Chanowitz and N. Fleishon, Phys. Lett. 82B (1979) 113; L. Bergström and G. Hulth, Nucl. Phys. B 259 (1985) 137 Erratum: [Nucl. Phys. B 276 (1986) 744].

[115] M. Spira, A. Djouadi and P. M. Zerwas, Phys. Lett. B 276 (1992) 350.

[116] R. Bonciani, V. Del Duca, H. Frellesvig, J. M. Henn, F. Moriello and V. A. Smirnov, JHEP 1508 (2015) 108; T. Gehrmann, S. Guns and D. Kara, JHEP 1509 (2015) 038.

[117] A. Abbasabadi, D. Bowser-Chao, D. A. Dicus and W. W. Repko, Phys. Rev. D 55 (1997) 5647; A. Abbasabadi and W. W. Repko, JHEP 0608 (2006) 048 and Phys. Rev. D 71 (2005) 017304; D. A. Dicus and W. W. Repko, Phys. Rev. D 87 (2013) no.7, 077301; L. B. Chen, C. F. Qiao and R. L. Zhu, Phys. Lett. B 726 (2013) 306; G. Passarino, Phys. Lett. B 727 (2013) 424.

[118] Y. Sun, H. R. Chang and D. N. Gao, JHEP 1305 (2013) 061.

[119] G. Gamberini, G. F. Giudice and G. Ridolfi, Nucl. Phys. B 292 (1987) 237.

[120] A. Brignole and F. Zwirner, Phys. Lett. B 299 (1993) 72; S. Heinemeyer and W. Hollik, Nucl. Phys. B 474 (1996) 32; K. E. Williams and G. Weiglein, Phys. Lett. B 660 (2008) 217; K. E. Williams, H. Rzehak and G. Weiglein, Eur. Phys. J. C 71 (2011) 1669; M. Krause, M. Mühlleitner, R. Santos and H. Ziesche, arXiv:1609.04185 [hep-ph].

[121] S. Heinemeyer, W. Hollik and G. Weiglein, Comput. Phys. Commun. 124 (2000) 76; T. Hahn, S. Heinemeyer, W. Hollik, H. Rzehak and G. Weiglein, Comput. Phys. Commun. 180 (2009) 1426.

[122] J. Baglio, R. Gröber, M. Mühlleitner, D. T. Nhung, H. Rzehak, M. Spira, J. Streicher and K. Walz, Comput. Phys. Commun. 185 (2014) no.12, 3372.

[123] A. G. Akeroyd, A. Arhrib and E. M. Naimi, Eur. Phys. J. C 12 (2000) 451 Erratum: [Eur. Phys. J. C 14 (2000) 371] and Eur. Phys. J. C 20 (2001) 51; M. Krause, R. Lorenz, M. Mühlleitner, R. Santos and H. Ziesche, JHEP 1609 (2016) 143.

[124] A. Djouadi, J. Kalinowski, P. Ohmann and P. M. Zerwas, Z. Phys. C 74 (1997) 93.

[125] A. Bartl, H. Eberl, K. Hidaka, T. Kon, W. Majerotto and Y. Yamada, Phys. Lett. B402 (1997) 303; A. Arhrib, A. Djouadi, W. Hollik and C. Jünger, Phys. Rev. D57 (1998) 5860.

[126] E. Accomando, G. Chachamis, F. Fugel, M. Spira and M. Walser, Phys. Rev. D 85 (2012) 015004. 
[127] H. Eberl, K. Hidaka, S. Kraml, W. Majerotto and Y. Yamada, Phys. Rev. D62 (2000) 055006.

[128] W. Frisch, H. Eberl and H. Hlucha, Comput. Phys. Commun. 182 (2011) 2219.

[129] C. Weber, K. Kovarik, H. Eberl and W. Majerotto, Nucl. Phys. B776 (2007) 138; S. Heinemeyer and C. Schappacher, Eur. Phys. J. C 75 (2015) no.5, 198.

[130] A. Djouadi, P. Janot, J. Kalinowski and P. M. Zerwas, Phys. Lett. B 376 (1996) 220.

[131] R. Y. Zhang, W. G. Ma, L. H. Wan and Y. Jiang, Phys. Rev. D 65 (2002) 075018; H. Eberl, W. Majerotto and Y. Yamada, Phys. Lett. B 597 (2004) 275; T. Ibrahim, Phys. Rev. D 76 (2007) 075012 and Phys. Rev. D 77 (2008) 065028; A. Bharucha, A. Fowler, G. Moortgat-Pick and G. Weiglein, JHEP 1305 (2013) 053; A. C. Fowler and G. Weiglein, JHEP 1001 (2010) 108; S. Heinemeyer and C. Schappacher, Eur. Phys. J. C 75 (2015) no.5, 230.

[132] E. Gross, G. Wolf and B. A. Kniehl, Z. Phys. C 63 (1994) 417 Erratum: [Z. Phys. C 66 (1995) 321]; A. Djouadi, M. Spira and P. M. Zerwas, Z. Phys. C 70 (1996) 427.

[133] L. G. Almeida, S. J. Lee, S. Pokorski and J. D. Wells, Phys. Rev. D 89 (2014) no.3, 033006; G. P. Lepage, P. B. Mackenzie and M. E. Peskin, arXiv:1404.0319 [hep-ph].

[134] N. Gray, D. J. Broadhurst, W. Grafe and K. Schilcher, Z. Phys. C 48 (1990) 673; K. G. Chetyrkin and M. Steinhauser, Phys. Rev. Lett. 83 (1999) 4001; K. G. Chetyrkin and M. Steinhauser, Nucl. Phys. B 573 (2000) 617; K. Melnikov and T. v. Ritbergen, Phys. Lett. B 482 (2000) 99; P. Marquard, A. V. Smirnov, V. A. Smirnov and M. Steinhauser, Phys. Rev. Lett. 114 (2015) no.14, 142002; A. L. Kataev and V. S. Molokoedov, Eur. Phys. J. Plus 131 (2016) no.8, 271.

[135] C. W. Bauer, Z. Ligeti, M. Luke, A. V. Manohar and M. Trott, Phys. Rev. D 70 (2004) 094017.

[136] K. A. Olive et al. [Particle Data Group Collaboration], Chin. Phys. C 38 (2014) 090001.

[137] M. Carena, S. Heinemeyer, O. Stal, C. E. M. Wagner and G. Weiglein, Eur. Phys. J. C 73 (2013) no.9, 2552.

[138] S. Dawson, Nucl. Phys. B 359 (1991) 283.

[139] D. Graudenz, M. Spira and P. M. Zerwas, Phys. Rev. Lett. 70 (1993) 1372; C. Anastasiou, S. Bucherer and Z. Kunszt, JHEP 0910 (2009) 068.

[140] M. Spira, Ph. D. Thesis, RWTH Aachen, 1992.

[141] J. Butterworth et al., J. Phys. G 43 (2016) 023001.

[142] S. Catani, D. de Florian and M. Grazzini, JHEP 0105 (2001) 025; R. V. Harlander and W. B. Kilgore, Phys. Rev. D 64 (2001) 013015 and Phys. Rev. Lett. 88 (2002) 201801; C. Anastasiou and K. Melnikov, Nucl. Phys. B 646 (2002) 220; V. Ravindran, J. Smith and W. L. van Neerven, Nucl. Phys. B 665 (2003) 325; S. Marzani, R. D. Ball, V. Del Duca, S. Forte and A. Vicini, Nucl. Phys. B 800 (2008) 127.

[143] T. Gehrmann, M. Jaquier, E. W. N. Glover and A. Koukoutsakis, JHEP 1202 (2012) 056; C. Anastasiou, C. Duhr, F. Dulat and B. Mistlberger, JHEP 1307 (2013) 003; C. Anastasiou, C. Duhr, F. Dulat, F. Herzog and B. Mistlberger, JHEP 1312 (2013) 088; W. B. Kilgore, Phys. Rev. D 89 (2014) 7, 073008; Y. Li, A. von Manteuffel, R. M. Schabinger and H. X. Zhu, Phys. Rev. D 90 (2014) 5, 053006; C. Anastasiou, C. Duhr, F. Dulat, E. Furlan, T. Gehrmann, F. Herzog 
and B. Mistlberger, JHEP 1503 (2015) 091; C. Anastasiou, C. Duhr, F. Dulat, F. Herzog and B. Mistlberger, Phys. Rev. Lett. 114 (2015) 21, 212001.

[144] C. Anastasiou, C. Duhr, F. Dulat, E. Furlan, T. Gehrmann, F. Herzog, A. Lazopoulos and B. Mistlberger, JHEP 1605 (2016) 058.

[145] M. Krämer, E. Laenen and M. Spira, Nucl. Phys. B 511 (1998) 523; S. Catani, D. de Florian, M. Grazzini and P. Nason, JHEP 0307 (2003) 028; S. Moch and A. Vogt, Phys. Lett. B 631 (2005) 48; V. Ravindran, Nucl. Phys. B 746 (2006) 58 and Nucl. Phys. B 752 (2006) 173; A. Idilbi, X. d. Ji, J. P. Ma and F. Yuan, Phys. Rev. D 73 (2006) 077501; V. Ahrens, T. Becher, M. Neubert and L. L. Yang, Eur. Phys. J. C 62 (2009) 333; D. de Florian and M. Grazzini, Phys. Lett. B 674 (2009) 291.

[146] D. de Florian, J. Mazzitelli, S. Moch and A. Vogt, JHEP 1410 (2014) 176; M. Bonvini and L. Rottoli, Phys. Rev. D 91 (2015) 5, 051301. S. Catani, L. Cieri, D. de Florian, G. Ferrera and M. Grazzini, Nucl. Phys. B 888 (2014) 75.

[147] D. de Florian and M. Grazzini, Phys. Lett. B 718 (2012) 117; M. Bonvini and S. Marzani, JHEP 1409 (2014) 007; T. Schmidt and M. Spira, Phys. Rev. D 93 (2016) no.1, 014022; M. Bonvini, S. Marzani, C. Muselli and L. Rottoli, JHEP 1608 (2016) 105.

[148] C. Anastasiou, R. Boughezal and F. Petriello, JHEP 0904 (2009) 003.

[149] R. V. Harlander and K. J. Ozeren, Phys. Lett. B 679 (2009) 467 and JHEP 0911 (2009) 088; A. Pak, M. Rogal and M. Steinhauser, Phys. Lett. B 679 (2009) 473 and JHEP 1002 (2010) 025.

[150] M. Spira, hep-ph/9510347 and Nucl. Instrum. Meth. A 389 (1997) 357.

[151] R. V. Harlander, S. Liebler and H. Mantler, Comput. Phys. Commun. 184 (2013) 1605 and arXiv:1605.03190 [hep-ph].

[152] C. Anastasiou, S. Buehler, F. Herzog and A. Lazopoulos, JHEP 1112 (2011) 058.

[153] M. Bonvini, ggHiggs, http://www.ge.infn.it/ bonvini/higgs/.

[154] M. Bonvini and L. Rottoli, TROLL, http://www.ge.infn.it/ bonvini/troll/.

[155] R. K. Ellis, I. Hinchliffe, M. Soldate and J. J. van der Bij, Nucl. Phys. B 297 (1988) 221; U. Baur and E. W. N. Glover, Nucl. Phys. B 339 (1990) 38.

[156] C. R. Schmidt, Phys. Lett. B 413 (1997) 391; D. de Florian, M. Grazzini and Z. Kunszt, Phys. Rev. Lett. 82 (1999) 5209; C. J. Glosser and C. R. Schmidt, JHEP 0212 (2002) 016; V. Ravindran, J. Smith and W. L. Van Neerven, Nucl. Phys. B 634 (2002) 247.

[157] R. V. Harlander, T. Neumann, K. J. Ozeren and M. Wiesemann, JHEP 1208 (2012) 139; T. Neumann and M. Wiesemann, JHEP 1411 (2014) 150.

[158] T. Neumann and C. Williams, arXiv:1609.00367 [hep-ph].

[159] R. Boughezal, F. Caola, K. Melnikov, F. Petriello and M. Schulze, JHEP 1306 (2013) 072 and Phys. Rev. Lett. 115 (2015) no.8, 082003; X. Chen, T. Gehrmann, E. W. N. Glover and M. Jaquier, Phys. Lett. B 740 (2015) 147; X. Chen, J. Cruz-Martinez, T. Gehrmann, E. W. N. Glover and M. Jaquier, JHEP 1610 (2016) 066. 
[160] Y. L. Dokshitzer, D. Diakonov and S. I. Troian, Phys. Rept. 58 (1980) 269; G. Parisi and R. Petronzio, Nucl. Phys. B 154 (1979) 427; G. Curci, M. Greco and Y. Srivastava, Nucl. Phys. B 159 (1979) 451; J. C. Collins and D. E. Soper, Nucl. Phys. B 193 (1981) 381 Erratum: [Nucl. Phys. B 213 (1983) 545] and Nucl. Phys. B 197 (1982) 446. J. Kodaira and L. Trentadue, Phys. Lett. 112B (1982) 66 and Phys. Lett. 123B (1983) 335; J. C. Collins, D. E. Soper and G. F. Sterman, Nucl. Phys. B 250 (1985) 199; S. Catani and M. Grazzini, Nucl. Phys. B 845 (2011) 297.

[161] G. Bozzi, S. Catani, D. de Florian and M. Grazzini, Nucl. Phys. B 737 (2006) 73;

[162] H. Mantler and M. Wiesemann, Eur. Phys. J. C 73 (2013) no.6, 2467; M. Grazzini and H. Sargsyan, JHEP 1309 (2013) 129; R. V. Harlander, H. Mantler and M. Wiesemann, JHEP 1411 (2014) 116; F. Caola, S. Forte, S. Marzani, C. Muselli and G. Vita, JHEP 1608 (2016) 150.

[163] E. Bagnaschi, R. V. Harlander, H. Mantler, A. Vicini and M. Wiesemann, JHEP 1601 (2016) 090 .

[164] G. Bozzi, S. Catani, D. de Florian and M. Grazzini, Nucl. Phys. B 791 (2008) 1.

[165] S. Frixione, P. Nason and C. Oleari, JHEP 0711 (2007) 070; S. Alioli, P. Nason, C. Oleari and E. Re, JHEP 1006 (2010) 043.

[166] E. Bagnaschi, G. Degrassi, P. Slavich and A. Vicini, JHEP 1202 (2012) 088.

[167] J. Alwall, M. Herquet, F. Maltoni, O. Mattelaer and T. Stelzer, JHEP 1106 (2011) 128; J. Alwall et al., JHEP 1407 (2014) 079.

[168] H. Mantler and M. Wiesemann, Eur. Phys. J. C 75 (2015) no.6, 257.

[169] D. A. Dicus and S. Willenbrock, Phys. Rev. D 39 (1989) 751.

[170] T. Han, G. Valencia and S. Willenbrock, Phys. Rev. Lett. 69 (1992) 3274.

[171] T. Figy, C. Oleari and D. Zeppenfeld, Phys. Rev. D 68 (2003) 073005; T. Figy and D. Zeppenfeld, Phys. Lett. B 591 (2004) 297; E. L. Berger and J. M. Campbell, Phys. Rev. D 70 (2004) 073011.

[172] V. N. Gribov and L. N. Lipatov, Sov. J. Nucl. Phys. 15 (1972) 438 [Yad. Fiz. 15 (1972) 781]; G. Altarelli and G. Parisi, Nucl. Phys. B 126 (1977) 298; Y. L. Dokshitzer, Sov. Phys. JETP 46 (1977) 641 [Zh. Eksp. Teor. Fiz. 73 (1977) 1216].

[173] M. Ciccolini, A. Denner and S. Dittmaier, Phys. Rev. Lett. 99 (2007) 161803 and Phys. Rev. D 77 (2008) 013002.

[174] A. Denner, S. Dittmaier, S. Kallweit and A. Mück, Comput. Phys. Commun. 195 (2015) 161.

[175] K. Arnold et al., Comput. Phys. Commun. 180 (2009) 1661, K. Arnold et al., arXiv:1107.4038 [hep-ph] and arXiv:1207.4975 [hep-ph]; J. Baglio et al., arXiv:1404.3940 [hep-ph].

[176] P. Bolzoni, F. Maltoni, S. O. Moch and M. Zaro, Phys. Rev. Lett. 105 (2010) 011801 and Phys. Rev. D 85 (2012) 035002.

[177] M. Cacciari, F. A. Dreyer, A. Karlberg, G. P. Salam and G. Zanderighi, Phys. Rev. Lett. 115 (2015) no.8, 082002. 
[178] R. V. Harlander, J. Vollinga and M. M. Weber, Phys. Rev. D 77, 053010 (2008); J. R. Andersen, T. Binoth, G. Heinrich and J. M. Smillie, JHEP 0802 (2008) 057; A. Bredenstein, K. Hagiwara and B. Jäger, Phys. Rev. D 77 (2008) 073004.

[179] F. A. Dreyer and A. Karlberg, Phys. Rev. Lett. 117 (2016) no.7, 072001.

[180] A. Djouadi and M. Spira, Phys. Rev. D 62 (2000) 014004.

[181] W. Hollik, T. Plehn, M. Rauch and H. Rzehak, Phys. Rev. Lett. 102 (2009) 091802; T. Figy, S. Palmer and G. Weiglein, JHEP 1202 (2012) 105.

[182] M. H. Seymour, Z. Phys. C 62 (1994) 127.

[183] T. Han and S. Willenbrock, Phys. Lett. B 273 (1991) 167.

[184] O. Brein, A. Djouadi and R. Harlander, Phys. Lett. B 579 (2004) 149.

[185] L. Altenkamp, S. Dittmaier, R. V. Harlander, H. Rzehak and T. J. E. Zirke, JHEP 1302 (2013) 078; A. Hasselhuhn, T. Luthe and M. Steinhauser, arXiv:1611.05881 [hep-ph].

[186] M. L. Ciccolini, S. Dittmaier and M. Krämer, Phys. Rev. D 68 (2003) 073003; A. Denner, S. Dittmaier, S. Kallweit and A. Mück, JHEP 1203 (2012) 075.

[187] G. Ferrera, M. Grazzini and F. Tramontano, JHEP 1404 (2014) 039 and Phys. Lett. B 740 (2015) 51.

[188] J. M. Campbell, R. K. Ellis and C. Williams, JHEP 1606 (2016) 179.

[189] O. Brein, R. V. Harlander and T. J. E. Zirke, Comput. Phys. Commun. 184 (2013) 998; R. V. Harlander, S. Liebler and T. Zirke, JHEP 1402 (2014) 023.

[190] W. Astill, W. Bizon, E. Re and G. Zanderighi, JHEP 1606 (2016) 154.

[191] W. Beenakker et al., Phys. Rev. Lett. 87 (2001) 201805.

[192] W. Beenakker et al., Nucl. Phys. B653 (2003) 151-203; L. Reina and S. Dawson, Phys. Rev. Lett. 87 (2001) 201804; S. Dawson, L. H. Orr, L. Reina, and D. Wackeroth, Phys. Rev. D67 (2003) 071503.

[193] R. Frederix, S. Frixione, V. Hirschi, F. Maltoni, R. Pittau and P. Torrielli, Phys. Lett. B 701 (2011) 427 .

[194] S. Dawson and L. Reina, Phys. Rev. D 57, 5851 (1998); E. Braaten and H. Zhang, Phys. Rev. D 93 (2016) no.5, 053014.

[195] M. V. Garzelli, A. Kardos, C. G. Papadopoulos and Z. Trocsanyi, Europhys. Lett. 96 (2011) 11001; H. B. Hartanto, B. Jäger, L. Reina and D. Wackeroth, Phys. Rev. D 91 (2015) no.9, 094003.

[196] F. Cascioli, S. Höche, F. Krauss, P. Maierhöfer, S. Pozzorini and F. Siegert, JHEP 1401 (2014) 046.

[197] A. Kulesza, L. Motyka, T. Stebel and V. Theeuwes, JHEP 1603 (2016) 065.

[198] A. Broggio, A. Ferroglia, B. D. Pecjak, A. Signer and L. L. Yang, JHEP 1603 (2016) 124; A. Broggio, A. Ferroglia, B. D. Pecjak and L. L. Yang, arXiv:1611.00049 [hep-ph]. 
[199] Y. Zhang, W. G. Ma, R. Y. Zhang, C. Chen and L. Guo, Phys. Lett. B 738 (2014) 1; S. Frixione, V. Hirschi, D. Pagani, H. S. Shao and M. Zaro, JHEP 1409 (2014) 065 and JHEP 1506 (2015) 184.

[200] A. Denner and R. Feger, JHEP 1511 (2015) 209.

[201] S. Dittmaier, M. Krämer and M. Spira, Phys. Rev. D 70 (2004) 074010; S. Dawson, C. B. Jackson, L. Reina and D. Wackeroth, Phys. Rev. D 69 (2004) 074027; M. Wiesemann, R. Frederix, S. Frixione, V. Hirschi, F. Maltoni and P. Torrielli, JHEP 1502 (2015) 132.

[202] D. L. Rainwater, M. Spira and D. Zeppenfeld, hep-ph/0203187; M. Spira, hep-ph/0211145; F. Maltoni, Z. Sullivan and S. Willenbrock, Phys. Rev. D 67 (2003) 093005; E. Boos and T. Plehn, Phys. Rev. D 69 (2004) 094005; J. M. Campbell et al., hep-ph/0405302.

[203] D. Dicus, T. Stelzer, Z. Sullivan and S. Willenbrock, Phys. Rev. D 59 (1999) 094016; C. Balazs, H. J. He and C. P. Yuan, Phys. Rev. D 60 (1999) 114001.

[204] R. V. Harlander and W. B. Kilgore, Phys. Rev. D 68 (2003) 013001.

[205] F. Maltoni, G. Ridolfi and M. Ubiali, JHEP 1207 (2012) 022 Erratum: [JHEP 1304 (2013) 095]; M. Lim, F. Maltoni, G. Ridolfi and M. Ubiali, JHEP 1609 (2016) 132.

[206] R. Harlander, M. Krämer and M. Schumacher, arXiv:1112.3478 [hep-ph].

[207] S. Forte, D. Napoletano and M. Ubiali, Phys. Lett. B 751 (2015) 331 and Phys. Lett. B 763 (2016) 190

[208] M. Bonvini, A. S. Papanastasiou and F. J. Tackmann, JHEP 1511 (2015) 196 and JHEP 1610 (2016) 053.

[209] F. Demartin, F. Maltoni, K. Mawatari and M. Zaro, Eur. Phys. J. C 75 (2015) no.6, 267.

[210] S. Dittmaier, P. Häfliger, M. Krämer, M. Spira and M. Walser, Phys. Rev. D 90 (2014) no.3, 035010 .

[211] P. Wu, W. G. Ma, H. S. Hou, R. Y. Zhang, L. Han and Y. Jiang, Phys. Lett. B 618 (2005) 209.

[212] S. Dawson, C. B. Jackson and P. Jaiswal, Phys. Rev. D 83 (2011) 115007.

[213] S. Dittmaier, M. Krämer, A. Mück and T. Schlüter, JHEP 0703 (2007) 114.

[214] P. Nason, S. Dawson and R. K. Ellis, Nucl. Phys. B 303 (1988) 607 and Nucl. Phys. B 327 (1989) 49 Erratum: [Nucl. Phys. B 335 (1990) 260]; W. Beenakker, H. Kuijf, W. L. van Neerven and J. Smith, Phys. Rev. D 40 (1989) 54; W. Beenakker, W. L. van Neerven, R. Meng, G. A. Schuler and J. Smith, Nucl. Phys. B 351 (1991) 507; M. Czakon, A. Mitov and S. Moch, Nucl. Phys. B 798 (2008) 210; P. Bärnreuther, M. Czakon and A. Mitov, Phys. Rev. Lett. 109 (2012) 132001; M. Czakon and A. Mitov, JHEP 1212 (2012) 054 and JHEP 1301 (2013) 080; M. Czakon, P. Fiedler and A. Mitov, Phys. Rev. Lett. 110 (2013) 252004; M. Czakon, P. Fiedler, D. Heymes and A. Mitov, JHEP 1605 (2016) 034.

[215] W. Beenakker, A. Denner, W. Hollik, R. Mertig, T. Sack and D. Wackeroth, Nucl. Phys. B 411 (1994) 343. 
[216] M. Jezabek and J. H. Kühn, Nucl. Phys. B 314 (1989) 1; C. S. Li, R. J. Oakes and T. C. Yuan, Phys. Rev. D 43 (1991) 3759; A. Czarnecki and S. Davidson, Phys. Rev. D 48 (1993) 4183; J. M. Campbell, R. K. Ellis and F. Tramontano, Phys. Rev. D 70 (2004) 094012; A. Czarnecki and K. Melnikov, Nucl. Phys. B 544 (1999) 520; K. G. Chetyrkin, R. Harlander, T. Seidensticker and M. Steinhauser, Phys. Rev. D 60 (1999) 114015; I. R. Blokland, A. Czarnecki, M. Slusarczyk and F. Tkachov, Phys. Rev. Lett. 93 (2004) 062001; I. R. Blokland, A. Czarnecki, M. Slusarczyk and F. Tkachov, Phys. Rev. D 71 (2005) 054004 Erratum: [Phys. Rev. D 79 (2009) 019901]; A. Czarnecki, J. G. Körner and J. H. Piclum, Phys. Rev. D 81 (2010) 111503; J. Gao, C. S. Li and H. X. Zhu, Phys. Rev. Lett. 110 (2013) no.4, 042001; M. Brucherseifer, F. Caola and K. Melnikov, JHEP 1304 (2013) 059.

[217] A. Denner and T. Sack, Nucl. Phys. B 358 (1991) 46; G. Eilam, R. R. Mendel, R. Migneron and A. Soni, Phys. Rev. Lett. 66 (1991) 3105; T. Kuruma, Z. Phys. C 57 (1993) 551.

[218] W. Peng, M. Wen-Gan, Z. Ren-You, J. Yi, H. Liang and G. Lei, Phys. Rev. D 73 (2006) 015012 Erratum: [Phys. Rev. D 80 (2009) 059901]; S. Dittmaier, M. Krämer, M. Spira and M. Walser, Phys. Rev. D 83 (2011) 055005.

[219] G. P. Gao, G. R. Lu, Z. H. Xiong and J. M. Yang, Phys. Rev. D 66 (2002) 015007; S. H. Zhu, Phys. Rev. D 67 (2003) 075006; T. Plehn, Phys. Rev. D 67 (2003) 014018; E. L. Berger, T. Han, J. Jiang and T. Plehn, Phys. Rev. D 71 (2005) 115012; N. Kidonakis, PoS HEP 2005 (2006) 336.

[220] M. Flechl, R. Klees, M. Krämer, M. Spira and M. Ubiali, Phys. Rev. D 91 (2015) no.7, 075015.

[221] C. Degrande, R. Frederix, V. Hirschi, M. Ubiali, M. Wiesemann and M. Zaro, arXiv:1607.05291 [hep-ph].

[222] K. D. Lane, eConf C 8206282 (1982) 222; E. Eichten, I. Hinchliffe, K. D. Lane and C. Quigg, Rev. Mod. Phys. 56 (1984) 579 Addendum: [Rev. Mod. Phys. 58 (1986) 1065]; N. G. Deshpande, X. Tata and D. A. Dicus, Phys. Rev. D 29 (1984) 1527.

[223] R. Foot, H. Lew and G. C. Joshi, Phys. Rev. D 37 (1988) 3161; S. Willenbrock, Phys. Rev. D35 (1987) 173; A. Krause, T. Plehn, M. Spira and P.M. Zerwas, Nucl. Phys. B519 (1998) 85; Y. Jiang, L. Han, W.G. Ma, Z.H. Yu and M. Han, J. Phys. G23 (1997) 385 [Erratum-ibid. G23 (1997) 1151]; Y. Jiang, W. g. Ma, L. Han, M. Han and Z. h. Yu, J. Phys. G 24 (1998) 83; O. Brein and W. Hollik, Eur. Phys. J. C13 (2000) 175.

[224] A.A. Barrientos Bendezu and B.A. Kniehl, Nucl. Phys. B568 (2000) 305.

[225] H. Hong-Sheng, M. Wen-Gan, Z. Ren-You, J. Yi, H. Liang and X. Li-Rong, Phys. Rev. D71 (2005) 075014; A. Alves and T. Plehn, Phys. Rev. D 71 (2005) 115014.

[226] A.A. Barrientos Bendezu and B.A. Kniehl, Phys. Rev. D59 (1999) 015009; O. Brein, W. Hollik and S. Kanemura, Phys. Rev. D63 (2001) 095001.

[227] A.A. Barrientos Bendezu and B.A. Kniehl, Phys. Rev. D 63 (2001) 015009.

[228] D.A. Dicus, J.L. Hewett, C. Kao and T.G. Rizzo, Phys. Rev. D 40 (1989) 787.

[229] W. Hollik and S.H. Zhu, Phys. Rev. D65 (2002) 075015; J. Zhao, C.S. Li and Q. Li, Phys. Rev. D72 (2005) 114008.

[230] S. Moretti, J. Phys. G 28 (2002) 2567. 
[231] E. Bagnaschi, R. V. Harlander, S. Liebler, H. Mantler, P. Slavich and A. Vicini, JHEP 1406 (2014) 167.

[232] G. Degrassi, P. P. Giardino, F. Maltoni and D. Pagani, arXiv:1607.04251 [hep-ph].

[233] J. Baglio, A. Djouadi, R. Gröber, M. M. Mühlleitner, J. Quevillon and M. Spira, JHEP 1304 (2013) 151.

[234] J. Baglio, A. Djouadi and J. Quevillon, Rept. Prog. Phys. 79 (2016) no.11, 116201.

[235] E. W. N. Glover and J. J. van der Bij, Nucl. Phys. B 309 (1988) 282; T. Plehn, M. Spira and P. M. Zerwas, Nucl. Phys. B 479 (1996) 46 Erratum: [Nucl. Phys. B 531 (1998) 655].

[236] S. Dawson, S. Dittmaier and M. Spira, Phys. Rev. D 58 (1998) 115012.

[237] J. Grigo, J. Hoff, K. Melnikov and M. Steinhauser, Nucl. Phys. B 875 (2013) 1.

[238] R. Frederix, S. Frixione, V. Hirschi, F. Maltoni, O. Mattelaer, P. Torrielli, E. Vryonidou and M. Zaro, Phys. Lett. B 732 (2014) 142; F. Maltoni, E. Vryonidou and M. Zaro, JHEP 1411 (2014) 079.

[239] G. Degrassi, P. P. Giardino and R. Gröber, Eur. Phys. J. C 76 (2016) no.7, 411; S. Borowka, N. Greiner, G. Heinrich, S. P. Jones, M. Kerner, J. Schlenk, U. Schubert and T. Zirke, Phys. Rev. Lett. 117 (2016) no.1, 012001 Erratum: [Phys. Rev. Lett. 117 (2016) no.7, 079901]; S. Borowka, N. Greiner, G. Heinrich, S. P. Jones, M. Kerner, J. Schlenk and T. Zirke, JHEP 1610 (2016) 107.

[240] D. de Florian and J. Mazzitelli, Phys. Lett. B 724 (2013) 306 and Phys. Rev. Lett. 111 (2013) 201801; J. Grigo, K. Melnikov and M. Steinhauser, Nucl. Phys. B 888 (2014) 17.

[241] J. Grigo, J. Hoff and M. Steinhauser, Nucl. Phys. B 900 (2015) 412.

[242] D. Y. Shao, C. S. Li, H. T. Li and J. Wang, JHEP 1307 (2013) 169; D. de Florian and J. Mazzitelli, JHEP 1509 (2015) 053.

[243] D. de Florian, M. Grazzini, C. Hanga, S. Kallweit, J. M. Lindert, P. Maierhöfer, J. Mazzitelli and D. Rathlev, JHEP 1609 (2016) 151.

[244] L. S. Ling, R. Y. Zhang, W. G. Ma, L. Guo, W. H. Li and X. Z. Li, Phys. Rev. D 89 (2014) no.7, 073001.

[245] H. T. Li and J. Wang, Phys. Lett. B 765 (2017) 265.

[246] M. Moretti, S. Moretti, F. Piccinini, R. Pittau and A. D. Polosa, JHEP 0502 (2005) 024.

[247] A. Agostini, G. Degrassi, R. Gröber and P. Slavich, JHEP 1604 (2016) 106. 